\title{
Analytical phase diagrams for colloids and non-adsorbing polymer
}

\author{
Gerard J. Fleer ${ }^{\mathrm{a}}$, Remco Tuinier ${ }^{\mathrm{b}, *}$ \\ ${ }^{a}$ Laboratory of Physical Chemistry and Colloid Science, Wageningen University, 6703 HB Wageningen, The Netherlands \\ b Forschungszentrum Jülich, Institut für Festkörperforschung, Soft Matter Group, 52425 Jülich, Germany
}

\section{A R T I C L E I N F O}

Available online 18 July 2008

\section{A B S T R A C T}

We review the free-volume theory (FVT) of Lekkerkerker et al. [Europhys. Lett. 20 (1992) 559] for the phase behavior of colloids in the presence of non-adsorbing polymer and we extend this theory in several aspects:

(i) We take the solvent into account as a separate component and show that the natural thermodynamic parameter for the polymer properties is the insertion work $\Pi v$, where $\Pi$ is the osmotic pressure of the (external) polymer solution and $v$ the volume of a colloid particle.

(ii) Curvature effects are included along the lines of Aarts et al. [J. Phys.: Condens. Matt. 14 (2002) 7551] but we find accurate simple power laws which simplify the mathematical procedure considerably.

(iii) We find analytical forms for the first, second, and third derivatives of the grand potential, needed for the calculation of the colloid chemical potential, the pressure, gas-liquid critical points and the critical endpoint (cep), where the (stable) critical line ends and then coincides with the triple point. This cep determines the boundary condition for a stable liquid.

We first apply these modifications to the so-called colloid limit, where the size ratio $q_{R}=R / a$ between the radius of gyration $R$ of the polymer and the particle radius $a$ is small. In this limit the binodal polymer concentrations are below overlap: the depletion thickness $\delta$ is nearly equal to $R$, and $\Pi$ can be approximated by the ideal (van 't Hoff) law $\Pi=\Pi_{0}=\varphi / N$, where $\varphi$ is the polymer volume fraction and $N$ the number of segments per chain. The results are close to those of the original Lekkerkerker theory. However, our analysis enables very simple analytical expressions for the polymer and colloid concentrations in the critical and triple points and along the binodals as a function of $q_{R}$. Also the position of the cep is found analytically. In order to make the model applicable to higher size ratio's $q_{R}$ (including the so-called protein limit where $q_{R}>1$ ) further extensions are needed. We introduce the size ratio $q=\delta / a$, where the depletion thickness $\delta$ is no longer of order $R$. In the protein limit the binodal concentrations are above overlap. In such semidilute solutions $\delta \approx \xi$, where the De Gennes blob size (correlation length) $\xi$ scales as $\xi \sim \varphi^{-\gamma}$, with $\gamma=0.77$ for good solvents and $\gamma=1$ for a theta solvent. In this limit $\Pi=\Pi_{\mathrm{sd}} \sim \varphi^{3 \gamma}$. We now apply the following additional modifications:

(iv) $\Pi=\Pi_{0}+\Pi_{\mathrm{sd}}$, where $\Pi_{\mathrm{sd}}=\mathrm{A} \varphi^{3 \gamma}$; the prefactor $\mathrm{A}$ is known from renormalization group theory. This simple additivity describes the crossover for the osmotic pressure from the dilute limit to the semidilute limit excellently.

(v) $\delta^{-2}=\delta_{0}^{-2}+\xi^{-2}$, where $\delta_{0} \approx R$ is the dilute limit for the depletion thickness $\delta$. This equation describes the crossover in length scales from $\delta_{0}$ (dilute) to $\xi$ (semidilute).

With these latter two modifications we obtain again a fully analytical model with simple equations for critical and triple points as a function of $q_{R}$. In the protein limit the binodal polymer concentrations scale as $q_{R}^{1 / \gamma}$, and phase diagrams $\varphi q_{R}^{-1 / \gamma}$ versus the colloid concentration $\eta$ become universal (i.e., independent of the size ratio $q_{R}$ ). The predictions of this generalized free-volume theory (GFVT) are in excellent agreement with experiment and with computer simulations, not only for the colloid limit but also for the protein limit (and the crossover between these limits). The $q_{R}^{1 / \gamma}$ scaling is accurately reproduced by both simulations and other theoretical models.

The liquid window is the region between $\varphi^{c}$ (critical point) and $\varphi^{t}$ (triple point). In terms of the ratio $\varphi^{t} / \varphi^{c}$ the liquid window extends from 1 in the $\operatorname{cep}\left(\right.$ here $\varphi^{t}-\varphi^{c}=0$ ) to 2.2 in the protein limit. Hence, the liquid window is narrow: it covers at most a factor 2.2 in (external) polymer concentration.

(c) 2008 Elsevier B.V. All rights reserved.

\footnotetext{
* Corresponding author. Current affiliation: DSM Research, ACES, P.O. Box 18, 6160 MD Geleen, The Netherlands.

E-mail addresses: remco.tuinier@googlemail.com, remco.tuinier@dsm.com (R. Tuinier).
} 


\section{Contents}

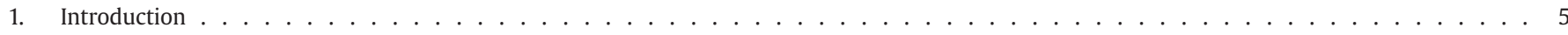

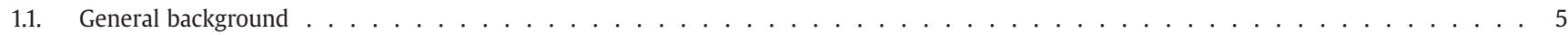

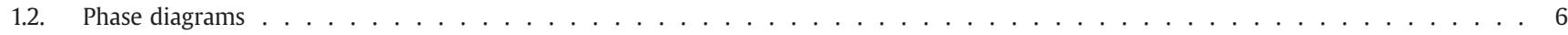

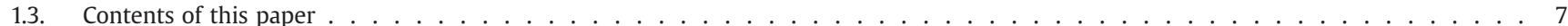

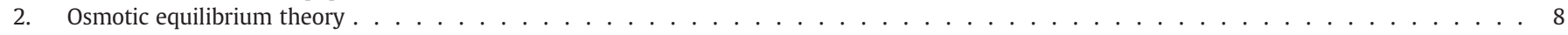

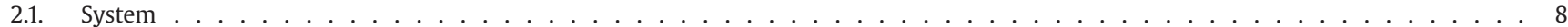

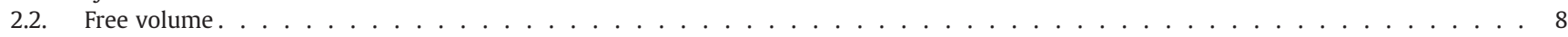

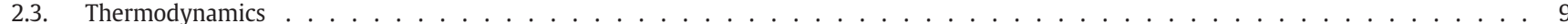

2.3.1. Separating hard-sphere and polymer contributions . . . . . . . . . . . . . . . . . . . . . . . . . 9

2.3.2. Hard-sphere contributions . . . . . . . . . . . . . . . . . . . . . . . . . . . . 9

2.3.3. Polymer contribution . . . . . . . . . . . . . . . . . . . . . . . . . . . . . . . . . . . . . 10

2.4. Binodals, critical and triple points, and critical endpoint . . . . . . . . . . . . . . . . . . . . . . . 11

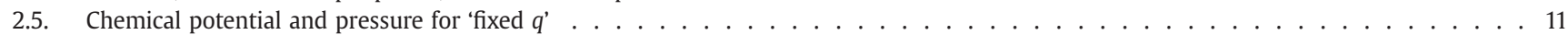

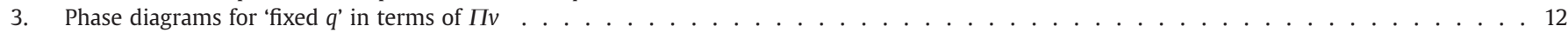

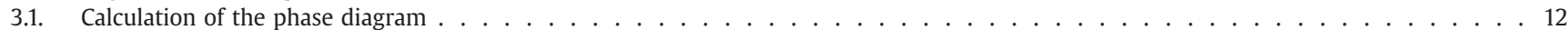

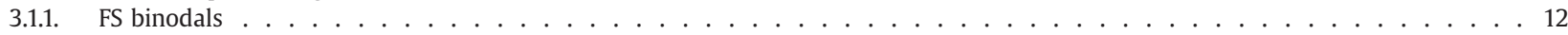

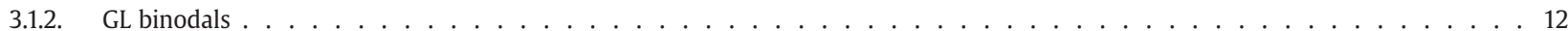

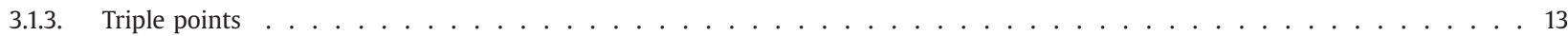

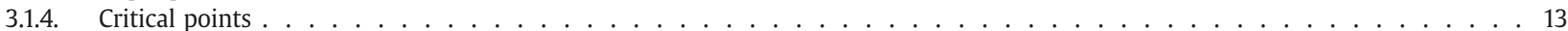

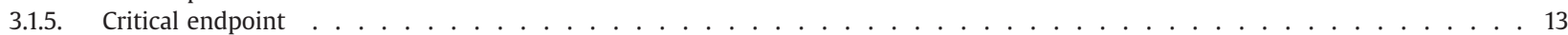

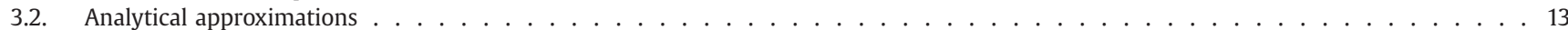

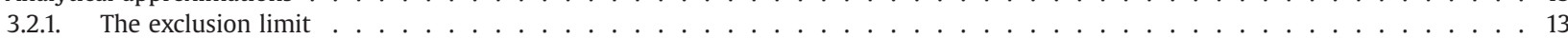

3.2.2. Power-law behavior of triple and critical points . . . . . . . . . . . . . . . . . . . . . . . . . . 14

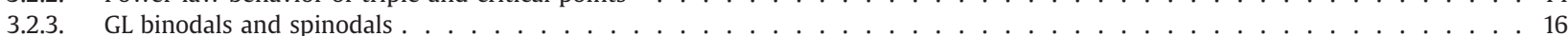

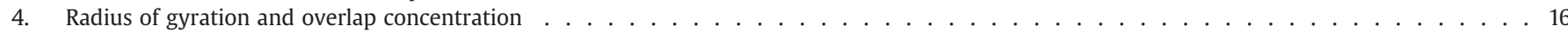

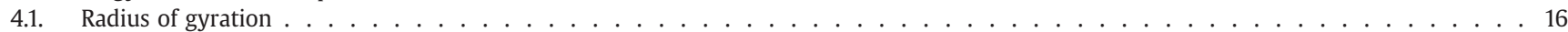

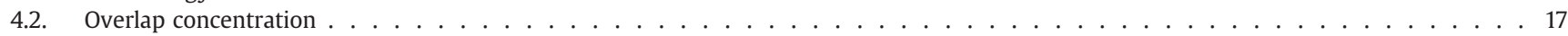

5. Phase diagrams for 'fixed $q$ ' in terms of the polymer concentration . . . . . . . . . . . . . . . . . . . . . . . . . . . . . . 18

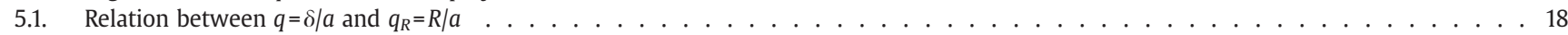

5.2. Relation between $\Pi v$ and reduced polymer concentration $y \ldots \ldots \ldots \ldots$

5.3. Phase diagrams in terms of the reduced polymer concentration $y \ldots \ldots \ldots \ldots$

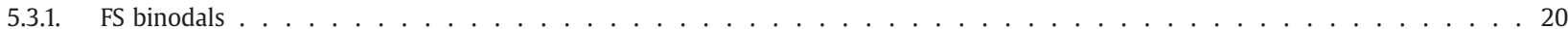

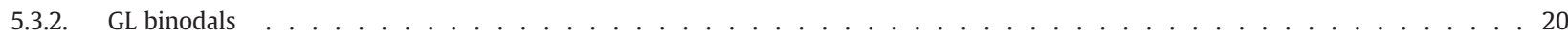

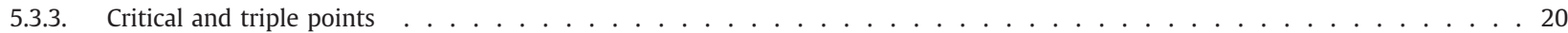

5.4. Phase diagrams in terms of the external polymer concentration $\varphi \ldots \ldots \ldots \ldots \ldots$

5.4.1. Phase diagrams at constant chain length $N \ldots \ldots \ldots \ldots \ldots$

5.4.2. Phase diagrams at constant particle radius $a \ldots \ldots \ldots \ldots \ldots$

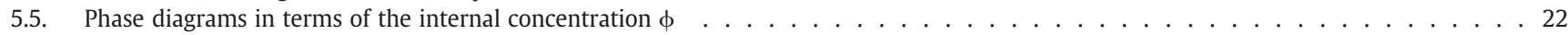

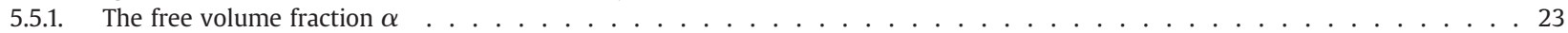

5.5.2. Critical and triple points in internal concentrations . . . . . . . . . . . . . . . . . . . . . . . . . . 24

5.5.3. Phase diagrams at constant chain length $N \ldots \ldots \ldots \ldots \ldots$

5.5.4. Phase diagrams at constant particle radius $a \ldots \ldots \ldots \ldots$

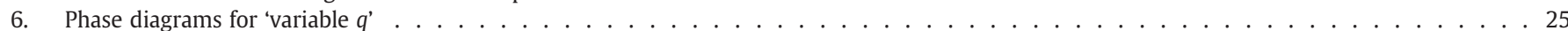

6.1. Concentration dependence of depletion thickness and osmotic pressure . . . . . . . . . . . . . . . . . . . . . . . . . . . 25

6.1.1. Depletion thickness in flat geometry . . . . . . . . . . . . . . . . . . . . . . . . . . 25

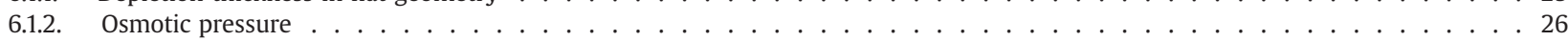

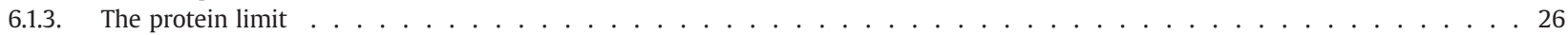

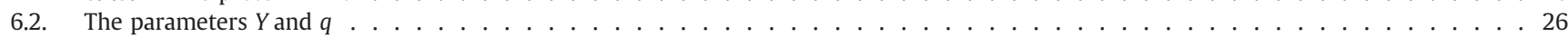

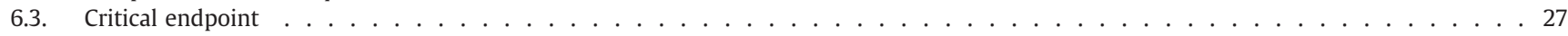

6.4. Triple and critical curves . . . . . . . . . . . . . . . . . . . . . . . . . . . . . 27

6.4.1. The parameters $Y^{c}$ and $Y^{t} \ldots \ldots \ldots \ldots \ldots \ldots \ldots \ldots$

6.4.2. The parameters $(\Pi v)^{c}$ and $(\Pi v)^{t} \ldots \ldots \ldots \ldots \ldots$

6.4.3. The parameters $q^{c}$ and $q^{t} \ldots \ldots \ldots \ldots \ldots$

6.4.4. The parameters $\mathrm{y}^{c}$ and $\mathrm{y}^{t} \ldots \ldots \ldots \ldots \ldots \ldots$

6.4.5. The parameters $\eta^{c}$ and $\eta^{t} \ldots \ldots \ldots \ldots \ldots \ldots$

6.4.6. The parameters $\alpha^{c}$ and $\alpha^{t} \ldots \ldots \ldots \ldots \ldots \ldots$

6.5. Phase diagrams in terms of the reduced polymer concentration $y \ldots \ldots \ldots \ldots$

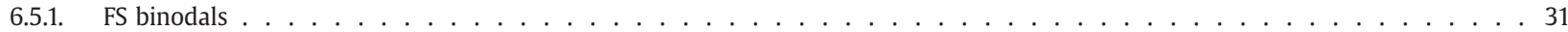

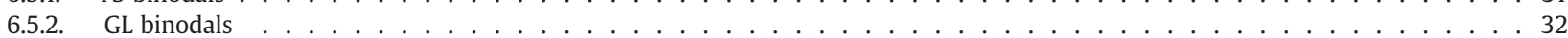

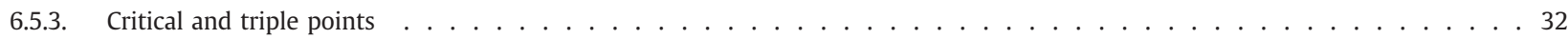

6.6. Phase diagrams in terms of the external polymer concentration $\varphi \ldots \ldots \ldots \ldots$

6.6.1. Phase diagrams at constant chain length $N \ldots \ldots \ldots \ldots$

6.6.2. Phase diagrams at constant particle radius $a \ldots \ldots \ldots$

6.7. Phase diagrams in terms of the internal concentration $\phi \ldots \ldots \ldots \ldots \ldots$

6.7.1. Critical and triple points in internal concentrations . . . . . . . . . . . . . . . . . . . . . . . . 34

6.7.2. Phase diagrams at constant chain length $N_{2} \ldots \ldots \ldots \ldots \ldots$

6.7.3. Phase diagrams at constant particle radius $a \ldots \ldots \ldots \ldots$

6.8. Analytical GL binodals . . . . . . . . . . . . . . . . . . . . . . . . . . . . . . . . 35

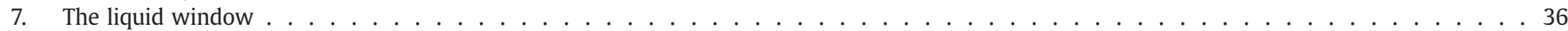

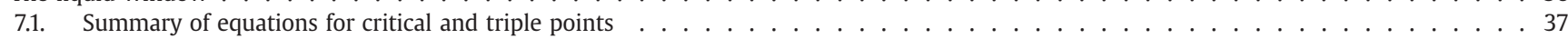


7.2. The contact potential . . . . . . . . . . . . . . . . . . . . . . . . . . . . . . . . . . . . 37

7.3. Analytical results for the liquid window . . . . . . . . . . . . . . . . . . . . . . . . . . . . . . . . . . . 38

7.3.1. The liquid window in terms of the interaction strength $\varepsilon$. . . . . . . . . . . . . . . . . . . . . . . . . . . . . 38

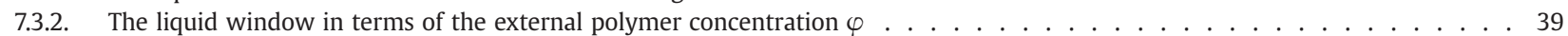

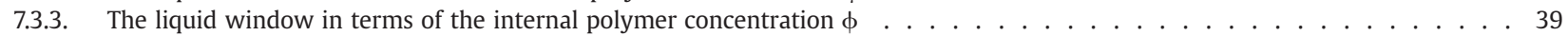

8. Comparison with experiments and simulations . . . . . . . . . . . . . . . . . . . . . . . . . . . . . . . . . . . . . . . . . . . 40

8.1. GL binodals from experiment . . . . . . . . . . . . . . . . . . . . . . . . . . . . . . . . . . . . . . 40

8.2. Critical points from experiment ... . . . . . . . . . . . . . . . . . . . . . . . . . . . . 41

8.3. GL binodals from simulations . . . . . . . . . . . . . . . . . . . . . . . . . . . . . . . . . . . . . . . . . . . . 42

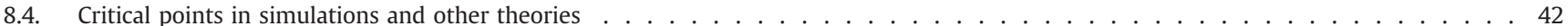

8.5. Free volume fraction . . . . . . . . . . . . . . . . . . . . . . . . . . . . . . . . . . . . . . 43

8.6. Tie lines . . . . . . . . . . . . . . . . . . . . . . . . . . . . . . . . . 43

8.7. Triple points . . . . . . . . . . . . . . . . . . . . . . . . . . . . . . . . . . . . . . . . . . . . 44

8.8. A complete phase diagram for $q_{R}=1$. . . . . . . . . . . . . . . . . . . . . . . . . . . . . . . . . . . . . . . . . . 44

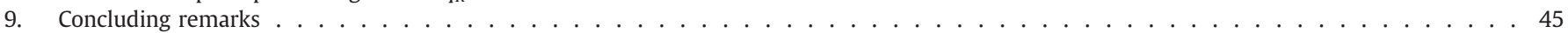

Acknowledgements . . . . . . . . . . . . . . . . . . . . . . . . . . . . . . . . . . . . . . . 46

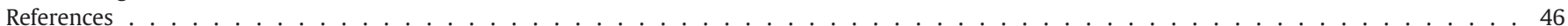

\section{Glossary of conventions and symbols}

\section{Units}

Unless indicated otherwise, all quantities in this paper are dimensionless, with the Kuhn length $\ell$ as the yardstick for all lengths, and kT for the energy. For example, the insertion work $\Pi v$ is in units $\mathrm{kT}$, and the radius of gyration $R$, the depletion thickness $\delta$, and the colloid radius $a$ are all in units $\ell$.

\section{Equations}

In the numbered equations, often two or more (sub)equations are combined, see for example Eq. (2.8) or Eq. (2.16). When we refer to a subequation in the text, we add the letter a for the first (Eq. (2.16a)), b for the second (Eq. (2.8b)), etc.

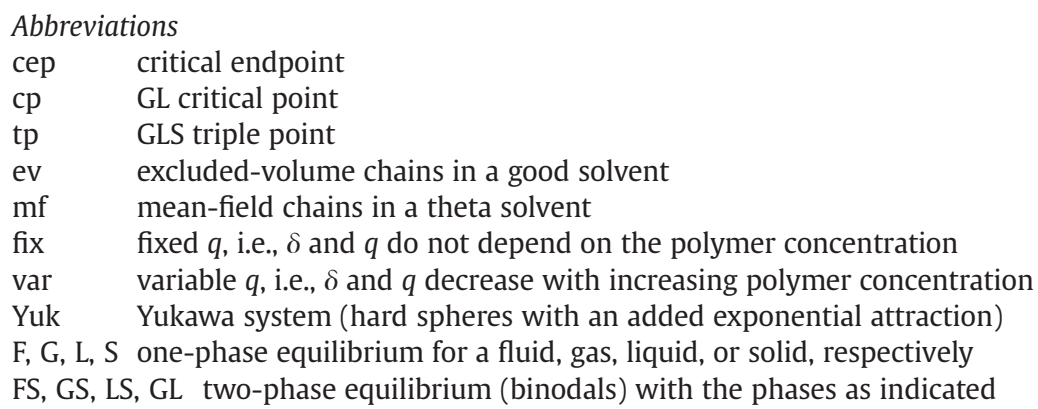

\section{Subscripts}

$0 \quad$ value in the limit of dilute polymer solutions

1 first derivative with respect to $f$

2 second derivative with respect to $f$

3 third derivative with respect to $f$

$\infty \quad$ value in the limit of high $q_{R}$

$f, g, l, s \quad$ value in fluid, gas, liquid, or solid phase

$\mathrm{p} \quad$ plate (only for $\delta_{\mathrm{p}}$ in Eqs. (6.5)-(6.7) and for $q_{\mathrm{p}}$ in Eqs. (6.7), (6.16), and (6.17))

$p \quad$ polymer

$s \quad$ solvent

$f s, g s, l s, g l$ value along the FS, GS, LS, GL binodals

ov overlap

sd semidilute limit

\section{Superscripts}

$0 \quad$ hard-sphere part of thermodynamic quantities $\Omega, \omega, \mu, p v$ [Eqs.(2.11), (2.13), (2.15)]

$p \quad$ polymer part of thermodynamic quantities $\Omega, \omega, \mu, p v$ [Eqs.(2.11), (2.13), (2.15)]

* $\quad$ value at cep (e.g., $q^{*}, q_{R}^{*}, \eta^{*}, \eta_{\mathrm{s}}^{*}, \varphi^{*},(\Pi v)^{*}$, etc $)$

$\sim \quad$ quantity is normalized on the cep, e.g., $\tilde{q}=q / q^{*}, \tilde{\eta}=\eta / \eta^{*}, \tilde{\varphi}=\varphi / \varphi^{*}$, etc.

c $\quad$ value at $\mathrm{cp}$

$t \quad$ value at tp

sp value at the (GL) spinodal 
Symbols (for several quantities the defining equation is given within square brackets)

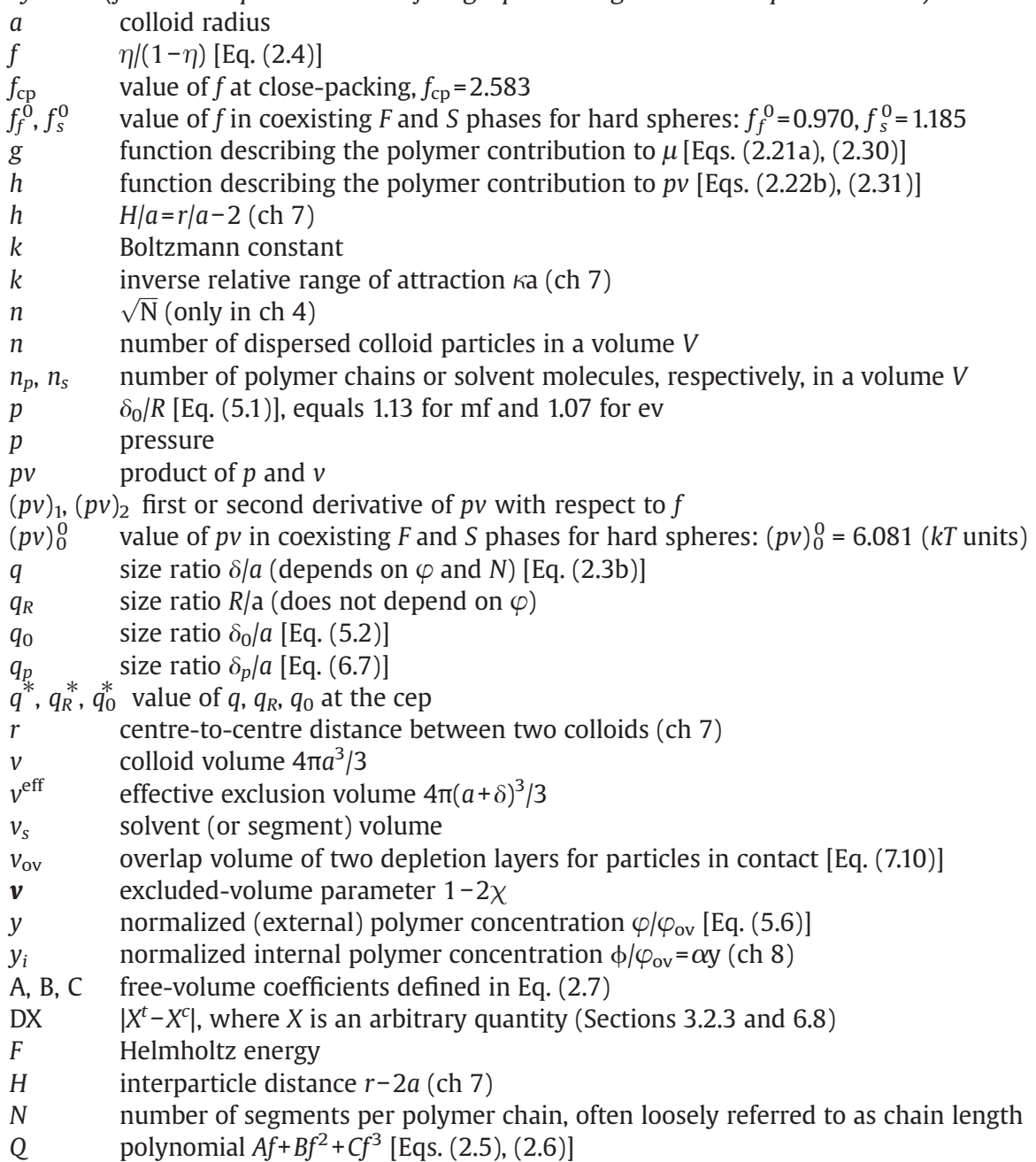

$Q_{1}, Q_{2}, Q_{3}$ first, second, third derivative of $Q$ with respect to $f$ [Eq. (2.8)]

$R \quad$ polymer radius of gyration

$S \quad$ entropy

$T \quad$ temperature

$V \quad$ volume

$V_{\text {coil }} \quad$ coil volume $4 \pi R^{3} / 3$

$W \quad$ pair potential (ch 7$)$

$Y \quad y q_{R}^{-1 / \gamma}$ [Eq. (6.15)]

$Y^{*} \quad$ value of $Y$ in the cep

$\alpha \quad$ free volume fraction [Eq. (2.1)]

$\alpha^{*}, \alpha_{s}^{*} \quad$ value of $\alpha$ in the fluid or solid phase at the cep

$\alpha_{\mathrm{e}} \quad$ linear expansion coefficient [Eq. (4.1)]

$\beta \quad \alpha /(1-\eta)$ [Eq. (2.5)]

$\beta_{1}, \beta_{2}, \beta_{3}$ first, second, third derivative of $\beta$ with respect to $f$ [Eq. (2.9)]

$\gamma \quad$ De Gennes exponent [Eq. (6.1)], equals $0.77 \mathrm{for}$ ev and 1 for $\mathrm{mf}$

$\delta \quad$ depletion thickness around a sphere; range of the depletion interaction

$\delta_{\mathrm{p}} \quad$ depletion thickness next to flat plate

$\delta_{0} \quad$ value of $\delta_{\mathrm{p}}$ in the dilute limit

$\varepsilon \quad$ interaction strength, pair potential for particles in contact (ch 7)

$\zeta \quad 1+q$ [Eq. (2.3a)], size ratio between a particle with and without depletion layer

$\eta \quad$ colloid volume fraction [Eq. (2.12b)], often loosely referred to as colloid concentration

$\eta^{*}, \eta_{s}^{*} \quad$ value of $\eta$ in the fluid or solid phase at the cep

$\eta_{f}^{0}, \eta_{s}^{0} \quad$ value of $\eta$ in coexisting $\mathrm{F}$ and S phases for hard spheres: $\eta_{f}^{0}=0.494, \eta_{\mathrm{s}}^{0}=0.545$

$\eta_{\mathrm{cp}} \quad$ value of $\eta$ at close-packing, $\eta_{\mathrm{cp}}=0.741$

$\kappa \quad$ inverse range of attraction (ch 7)

$\mu \quad$ colloid chemical potential

$\mu_{p}, \mu_{s} \quad$ chemical potential of polymer and solvent, respectively 


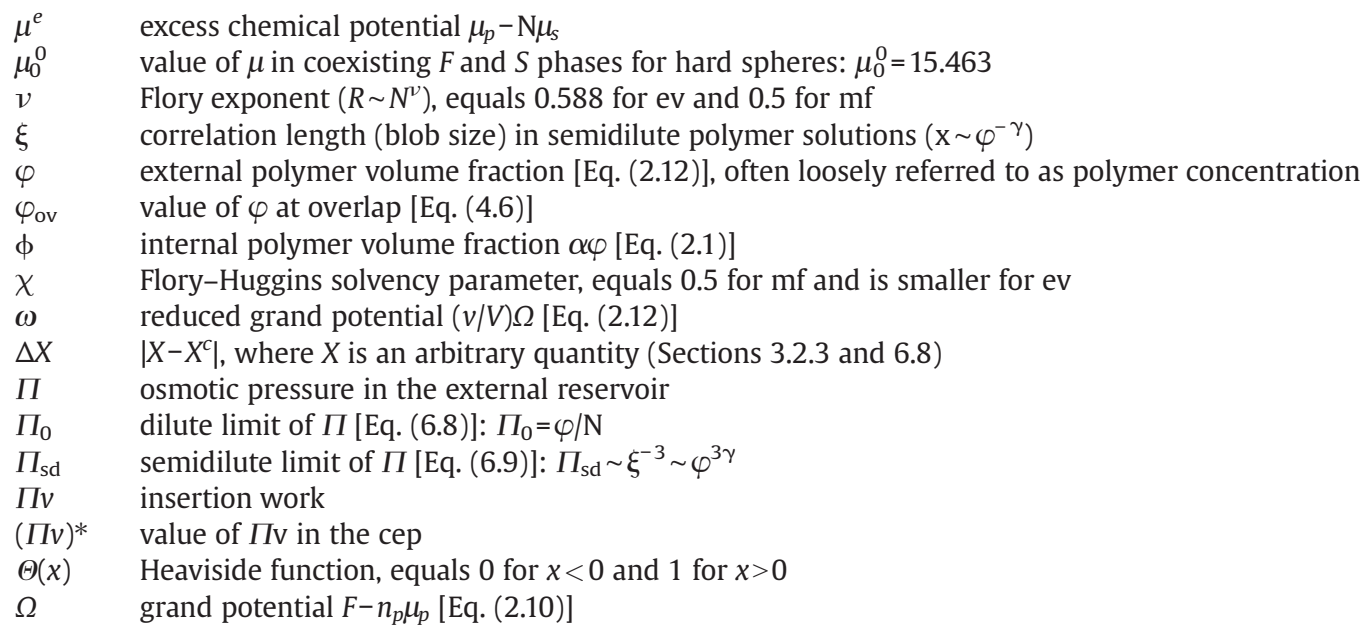

\section{Introduction}

\subsection{General background}

In the beginning of the previous century there was a lively debate on whether atoms exist. Various theoretical predictions for atomic systems were made but could not yet be tested experimentally. The first indications for the particulate nature of matter came from the field of colloids. Einstein [1] showed that dilute colloidal particles in a solvent should obey the gas law. Von Smoluchowski [2] predicted that colloidal particles exhibit Brownian motion, just like atoms in a gas. Perrin [3] proved that matter is particulate by studying colloidal resin particles: he found excellent agreement with the gas law and visualized the Brownian motion of colloidal particles. Perrin's resin colloids followed the heightdistribution according to Boltzmann's law in a gravity field.

An essential difference between atomic and colloidal fluids is that the pair interactions between atoms are fixed (they are determined by quantum mechanics), whereas those between colloids can, in principle, be adjusted [4]. A controlled way of increasing the attractive forces between colloids is by adding non-adsorbing polymer chains. When the attraction is strong enough (i.e., above a certain polymer concentration) phase transitions similar to those in atomic systems may then occur $[5,6]$.

Over the last decades, the phase behavior of mixtures of colloids and non-adsorbing polymers has gained substantial practical and fundamental interest from both experimentalists [7-9] and theoreticians [1016]. Non-adsorbing polymers induce a so-called depletion force between the colloidal particles, leading to an effective attraction due to an unbalanced osmotic pressure, as first realized by Asakura and Oosawa $[17,18]$ half a century ago. Shortly after, Sieglaff [19] succeeded in explaining his findings for the demixing of polystyrene chains and colloidal microgel particles in toluene on the basis of this depletion principle.

Vrij $[20,21]$ made the first attempts to describe this phase behavior theoretically. He simplified the polymer chains as Freely Overlapping Spheres (FOS's) which are impenetrable for the colloidal hard spheres (HS's) but can freely overlap other FOS's. He used the resulting depletion pair interaction between the colloids to predict the stability regions in the phase diagram of such colloid-polymer mixtures in a simple analysis based upon the second virial coefficient. Early experiments by De Hek and Vrij [21] and Vincent et al. [22,23] confirmed the general concepts.

A few years later, Gast et al. [24] constructed a pair-wise perturbation theory for the Helmholtz energy of a mixture of HS's plus FOS's, which enabled the computation of the colloid volume fractions in the coexisting phases at given external FOS concentration. An osmotic equilibrium or free volume theory (FVT) [25], in which polymer partitioning over the coexisting phases is taken into account, was later developed by Lekkerkerker et al. [26]. This theory gives a fair description of the phase behavior of model systems of colloidal dispersions of (pseudo)hard spheres mixed with well-defined synthetic polymer chains, as long as the polymer chains are small compared to the colloids (the so-called colloid limit, where the binodal polymer concentrations are below overlap) [27]. The theory compares well with computer simulations of HS's plus ideal chains [10] or HS's plus FOS's [11,28,29].

In recent years, there have been significant theoretical developments which take into account the polymeric excluded-volume interactions [30-41]. As examples of methods that enable the prediction of the phase behavior of colloid-polymer mixtures we mention polymer-colloid liquid state theory [31-34], thermodynamic perturbation theories [12,35], density functional theory [36,37], a Gaussian-core model [14,38,39], and computer simulations of HS plus self-avoiding polymer chains $[40,41]$. To obtain phase lines these methods require a significant amount of numerical work.

The osmotic equilibrium theory by Lekkerkerker et al. [26] for the phase behavior of dispersions of colloids and non-adsorbing polymer is much simpler in this respect; it serves as a standard reference today. The starting point is the grand potential density of a system of colloids and polymer in equilibrium with a reservoir containing only the polymer. This grand potential is separated into a HS contribution and a polymer contribution. The HS part may be described by known expressions for the colloidal fluid and crystalline phases. The polymer contribution is found from a build-up principle: starting from a system without polymer, chains are added to the system until the final concentration is reached, and the polymer contribution is calculated by integrating along this path.

The original version of the Lekkerkerker theory was formulated for ideal chains, for which the depletion thickness $\delta$ is taken to be equal to the radius of gyration $\mathrm{R}$ of the polymer chains and for which the polymer osmotic pressure is given by the ideal (Van 't Hoff) contribution $\Pi=\varphi / N$. Here $\varphi$ is the polymer (segment) volume fraction in the external reservoir and $\mathrm{N}$ is the number of segments per chain, and $\Pi$ is expressed in units $\mathrm{kT} / \mathscr{\ell}^{3}$, where $\ell$ is the Kuhn length. The parameters entering the model are the colloid volume fraction $\eta$ and two ratios related to the polymer properties: the ratio $q_{R}=R / a$, where $a$ is the colloid radius, and the ratio $y=\varphi / \varphi_{\mathrm{ov}}$, where $\varphi_{\mathrm{ov}}$ is the overlap concentration.

This simple model is a fair approximation for the colloid limit $(R<a$, $q_{R}<1$ ) where the polymer concentrations at phase coexistence are below overlap $(y<1)$ and where the depletion thickness $\delta \approx R$ is constant; this is the appropriate polymer length scale in dilute solutions. The model breaks down in the so-called protein limit $(R>a$, $q_{R}>1$ ) where the binodal concentrations are in the semidilute regime 


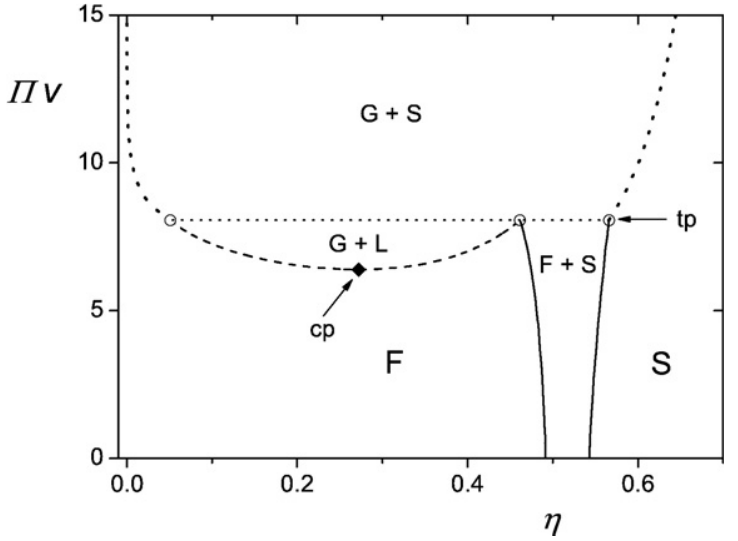

Fig. 1.1. GL binodal (dashed), GS binodal (dotted) and FS binodal (solid) for $q=0.4$ in a $\Pi v(\eta)$ representation. The diamond is the GL critical point, the circles connected through the horizontal dotted line give the triple point. The demixing regions are indicated as $\mathrm{G}+\mathrm{S}, \mathrm{G}+\mathrm{L}$, and $\mathrm{F}+\mathrm{S}$. Also the one-phase regions for a fluid (F) and a solid (S) are shown.

$(y>1)$ and where the polymer length scale is the correlation length (blob size) $\xi$, which is independent of chain length and is only a (power-law) function of $y$. For this limit no satisfactory theory for binodal curves exists so far. We shall present one in chapter 6 , and we treat also the crossover between the colloid and protein limits. Short accounts of this new theory have been published recently $[42,43]$.

For extending the Lekkerkerker theory one has to account for several factors. The first is that, even in the colloid limit (and even for ideal chains), the depletion thickness $\delta_{0}$ next to a planar surface (or around a large sphere) is somewhat larger than $R$. The second is due to curvature effects: the depletion thickness $\delta$ around a sphere is smaller than $\delta_{0}$. The third is to incorporate non-ideal contributions to the osmotic pressure $\Pi$ of the polymer, which show up especially when $\varphi$ is of order $\varphi_{\mathrm{ov}}$ or above (i.e., in the protein limit) and which make $\Pi$ (much) larger than the ideal contribution $\Pi_{0}=\varphi / \mathrm{N}$. Finally, also for the depletion thickness such non-ideal effects play a role: the depletion thickness $\delta$ next to a plate decreases with increasing polymer concentration, from the chain-length dependent length scale $\delta_{0} \approx R$ in the dilute limit $(y \rightarrow 0)$ to the concentration-dependent length scale $\xi$ in semidilute solutions $(y>>1)$.

Aarts et al. [30] were the first to incorporate these effects into the osmotic equilibrium theory. They used (complicated) expressions derived from renormalization group (RG) theory [44] for the dependences $\Pi / \Pi_{0}$ and $\delta / \delta_{0}$ on the ratio $y=\varphi / \varphi_{\mathrm{ov}}$, and also accounted for $\delta_{0} / R \neq 1$ and for curvature effects. They presented a few examples of phase diagrams for polymer chains in the excluded-volume limit and calculated (numerically) gas-liquid critical points and gas-liquidsolid triple points. An important result of their work is that even in this more sophisticated model the same three parameters $\eta, q_{R}=R / a$, and $y=\varphi / \varphi_{\text {ov }}$ are sufficient to describe the phase behavior. The authors did not address the conversion from the normalized parameters $q_{R}$ and $y$ towards polymer chain length and polymer concentration. For this conversion information about the dependence of $R$ and $\varphi_{\mathrm{ov}}$ on chain length and solvency is needed. Moreover, their thermodynamic treatment (like that in the original Lekkerkerker theory) is incomplete in so far that the solvent is treated as background, and not taken into account as a separate component. In addition, their correlation length in semidilute polymer solutions is too small [44].

In this paper we review the thermodynamic basis of the osmotic equilibrium theory and we extend this theory in several aspects. The most important feature is that we shall deal with the crossover in length scales, from coil size $R$ to blob size $\xi$, which extends the validity of the osmotic equilibrium theory towards the protein limit. Another aspect is that we explicitly account for the solvent component. The solvent chemical potential is directly related to the osmotic pressure $\Pi$ of the polymer solution. We show that the product $\Pi v$, where $v=$
$(4 \pi / 3) a^{3}$ is the colloid volume, is the natural parameter for describing the thermodynamics. The thermodynamic parameters are then the colloid concentration $\eta$, the effective size ratio $q=\delta / a$, and the product $\Pi v$, which is the osmotic work of inserting a colloid particle (without depletion layer) into the polymer solution. We will show that $q$ and $\Pi v$ can be simply expressed in the ratios $y=\varphi / \varphi_{\mathrm{ov}}$ and $q_{R}=R / a$.

\subsection{Phase diagrams}

In Figs 1.1 and 1.2 we illustrate the basic features of $\Pi v(\eta)$ phase diagrams for the colloid limit; at this stage we still assume that the depletion thickness $\delta$ (and, hence, $q=\delta / a$ ) does not depend on polymer concentration. We will see later that in the general case where $\delta$ depends on the polymer concentration the qualitative features are the same (and the quantitative differences in a $\Pi v(\eta)$ representation are small).

Fig. 1.1 shows an example of a GL binodal (dashed), a GS binodal (dotted) and an FS binodal (solid); in this example $q=0.4$. The GL binodal is only stable in a relatively small $\Pi v$ window, in between the lowest value $(\Pi v)^{c}$ corresponding to the GL critical point (cp, indicated by the diamond) and the highest value $(\Pi v)^{t}$ corresponding to the triple point (tp, the three open circles connected by the horizontal dotted line). Above the triple point there is GS equilibrium between a (very) dilute gas phase and a concentrated solid phase. The FS binodal is stable for $\Pi v<(\Pi v)^{t}$ and the coexistence concentrations are close to the well-known HS coexistence concentrations $\eta=0.49$ (liquid) and $\eta=0.54$ (solid); the pressure $p$ at this HS coexistence (see Section 2.3.2) corresponds to $p v=6.08$ ( $k T$ units).

Fig. 1.1 applies to one particular size ratio $q=\delta / a=0.4$. Fig. 1.2 demonstrates how the coordinates of the critical point (cp) and the triple point (tp) vary with $q$. The diamond (cp) and circles (tp) are the same as in Fig. $1.1(q=0.4)$. As $q$ decreases (in the direction of the arrows), $(\Pi v)^{c}$ and $\eta^{c}$ increase, as shown by the critical curve (label cp) in Fig. 1.2. This figure also shows the triple curve (label tp) which, obviously, has three branches for the three coexisting phases. Each triple point at given $q$ is characterized by four coordinates: $(\Pi v)^{t}$ and three compositions $\eta_{g}^{t}, \eta_{l}^{t}$, and $\eta_{s}^{t}$. For high $q$ (above 0.6 ), where the polymer is essentially absent from the condensed phases, $(\Pi v)^{t}$ equals 6.08 (dashed line in Fig. 1.2), which is the value of $p v$ in a HS system without polymer; $(\Pi v)^{t}$ cannot drop below this value.

With decreasing $q$, the fluid compositions $\eta_{g}^{t}$ and $\eta_{l}^{t}$ of the triple point approach each other; the liquid window narrows. At a critical value $q^{*}$ (about $1 / 3$ ) the values of $\eta_{g}^{t}$ and $\eta_{l}^{t}$ merge at the critical value

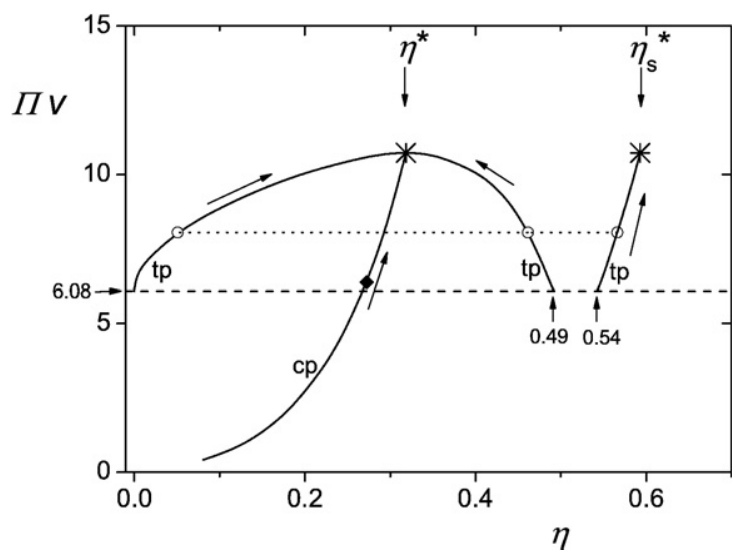

Fig. 1.2. Variation of the critical point (cp) and of the triple point (tp) with the size ratio $q=\delta / a$. The arrows indicate the direction of decreasing $q$. The diamond and the circles (connected by the dotted line) are the same $(q=0.4)$ as in Fig. 1.1. The asterisks correspond to the critical endpoint (cep), where a critical fluid at concentration $\eta^{*}$ (left asterisk) coexists with a solid at concentration $\eta_{s}^{*}$ (right asterisk); this cep is reached at $q \approx 1 / 3$. The fluid part of the cep is the endpoint of (the stable part of) the critical curve. The high- $q$ limit of the triple point is $\Pi v=6.08$, indicated as the dashed line; the liquid and solid compositions in this limit are equal to the hard-sphere coexistence concentrations 0.49 and 0.54 , respectively. 
$\eta^{*}$, which marks the endpoint of the stable part of the critical curve (left asterisk in Fig. 1.2). At that point there is equilibrium with a solid phase of composition $\eta_{s}^{*}$ (right asterisk). The two asterisks in Fig. 1.2 correspond to the critical endpoint (cep), which is a central feature in the phase diagram because it constitutes the boundary condition for the existence of a stable liquid phase. This cep is again characterized by four coordinates: $q^{*},(\Pi v)^{*}, \eta^{*}$, and $\eta_{s}^{*}$.

\subsection{Contents of this paper}

In chapter 2 we (re)formulate the thermodynamic background of the osmotic equilibrium theory in terms of the parameters $\eta, q=\delta / a$, and $\Pi v$. The treatment is general in the sense that $\delta$ and $q$ may depend on the polymer concentration, and $\Pi$ and $\delta$ may contain nonideal contributions. We present analytical expressions for the colloid chemical potential $\mu$ and the product $p v$, where $p$ is the pressure of the system; both $\mu$ and $p v$ have a hard-sphere part and a polymer contribution (defined in terms of $\Pi \mathrm{v}$ ). We find also analytical expressions for the first and second derivatives of $p v$ with respect to $\eta$; these derivatives are needed for calculating GL critical points. We then show how - in the general case - binodals are found from solving two equations in two unknowns (the coexisting compositions). Also the calculation of GL critical points requires solving two equations in two unknowns (in this case $(\Pi v)^{c}$ and $\eta^{c}$ ). For the triple points we have to solve four equations in four unknowns: $(\Pi v)^{t}$ and three coexisting compositions. Finally, also the critical endpoint follows from four equations in the four unknowns $q^{*},(\Pi \mathrm{v})^{*}, \eta^{*}$, and $\eta_{s}^{*}$.

For the special case of a constant polymer length scale (hence, $\delta$ and $q$ are independent of the polymer concentration or $\Pi v$ ), the equations may be simplified. We denote this situation as 'fixed q'. Now the calculation of all the characteristic points (binodal points, critical points, triple point, critical endpoint) may be reduced to solving one equation in one unknown and a fully analytical phase diagram can be obtained. This situation is discussed in chapter 3. It is found that the cep is situated at $q^{*}=0.328,(\Pi v)^{*}=10.73, \eta^{*}=0.319$, and $\eta_{s}^{*}=0.593$. We show that the essential features of the phase diagram (critical curve, triple curve) may be approximated quite well through power laws in the reduced quantities $\tilde{\eta}=\eta / \eta^{*}, \widetilde{\Pi v}=\Pi v /(\Pi v)^{*}$, and $\tilde{q}=q / q^{*}$, obtained by normalizing on the cep. This again illustrates the central role of the cep in phase diagrams. Throughout this paper we use* (asterisk) to denote the cep and (tilde) to indicate that the quantity of interest is normalized on the cep.

The parameter $q$ may be simply related to the size ratio $q_{R}=R / a$; for 'fixed $q$ ' the approximate relation is $q \approx 0.9 q_{R}^{0.9}$, which accounts for curvature effects. The parameter $\Pi v$ can be expressed in $y=\varphi / \varphi_{\mathrm{ov}}$ and $q_{R}$ in a very simple way: $\Pi v=q_{R}^{-3} y$ in dilute solutions. Hence, $\Pi v(\eta)$ diagrams for various $q$ may be converted to $y(\eta)$ diagrams for various $q_{R}$. This conversion is discussed in chapter 5 . In order to convert the normalized parameter $y$ to the real concentration $\varphi$ for a given polymer chain length $N$ and solvency $\boldsymbol{v}=1-2 \chi$ (where $\chi$ is the FloryHuggins parameter) one has to know how the overlap concentration $\varphi_{\mathrm{ov}}$ and the radius of gyration $R$ depend on $N$ and $\boldsymbol{v}$. Preceding the discussion of $y(\eta)$ phase diagrams in chapter 5 , we address this issue in chapter 4 . We present explicit expressions for $\varphi_{\mathrm{ov}}(N, \boldsymbol{v})$ and $R(N, \boldsymbol{v})$ for mean-field $(\mathrm{mf})$ chains in a theta solvent $(\boldsymbol{v}=0)$ and for excludedvolume (ev) chains in a good solvent $(\boldsymbol{v}>0)$, and we give useful approximate scaling relations (including the numerical prefactors) for these dependencies. Whenever we use the abbreviation $\mathrm{mf}$ we refer to a theta solvent, while ev refers to a good solvent.

As stated above, chapter 5 gives $y(\eta)$ and $\varphi(\eta)$ phase diagrams for 'fixed $q$ '. The cep turns out to be situated around $y^{*}=0.35$, which is indeed well below overlap $(y=1)$ : for the region around the cep the approximation of a constant $q$ is thus reasonable. For the phase diagrams $\varphi(\eta)$ we distinguish between two cases. The first is constant $R$, so $q$ is varied by adjusting the particle radius $a$. In this case $\varphi_{\text {ov }}$ does not depend on $q$, and is constant across the entire phase diagram; then $\varphi / \varphi^{*}=y / y^{*}$ or $\tilde{\varphi}=\tilde{y}$. The second situation is that of constant particle radius a, whereby $\mathrm{q}$ is varied by changing $R$. Now $\varphi_{\mathrm{ov}}$ is a function of $q$ and the relation between $\varphi$ and $y$ is slightly more complicated. To a good approximation it is $\tilde{\varphi}=\tilde{y} \tilde{q}_{R}^{-1}(\mathrm{mf})$ or $\tilde{\varphi}=\tilde{y} \tilde{q}_{R}^{-4 / 3}(\mathrm{ev})$. For both situations (constant $R$ and constant $a$ ) we give simple analytical expressions $\varphi\left(q_{R}\right)$ for critical and triple points.

In the final section of chapter 5 we also discuss $\phi(\eta)$ phase diagrams in terms of the internal concentrations $\phi=\alpha \varphi$, where $\alpha$ is the fraction of free volume in each phase. This fraction $\alpha$ depends strongly on the colloid concentration. One of the implications is that, whereas in terms of $\Pi v$ or the external concentration $\varphi$ the triple point may be represented as a horizontal line (see Fig. 1.1), the internal concentration $\phi^{t}$ differs strongly in the three coexisting phases, and the horizontal triple line is converted into a triple triangle.

Chapter 6 constitutes the most important part of this paper. We introduce a concentration-dependent polymer length scale, which enables the calculation of phase diagrams for any polymer concentration, including the semidilute limit (which corresponds to the protein limit). The depletion thickness $\delta$ decreases from $\delta=\delta_{0} \approx R$ in dilute solutions towards it semidilute limit $\delta=\xi \sim \varphi^{-\gamma}$, where $\xi$ is the correlation length (blob size) and $\gamma$ is the De Gennes exponent which equals 1 in a theta solvent ( $\mathrm{mf}$ ) and 3/4 (or 0.77) in good solvents (ev). Simultaneously, the osmotic pressure $\Pi$ displays a crossover from the dilute limit $\Pi=\Pi_{0}=\varphi / N$ towards the semidilute limit $\Pi=\Pi_{\mathrm{sd}} \sim \xi^{-3} \sim \varphi^{3 \gamma}$. This situation is denoted as 'variable $q$ '.

We employ very simple - yet accurate - expressions proposed recently $[45,46]:\left(\delta_{0} / \delta\right)^{2}=1+c_{1} y^{2 \gamma}$ and $\Pi / \Pi_{0}=1+c_{2} y^{3 \gamma-1}$, where $c_{1}$ and $c_{2}$ are known constants of order unity. Clearly, in semidilute solutions these expressions reduce to $\delta_{\mathrm{sd}} \sim \varphi^{-\gamma}$ and $\Pi_{\mathrm{sd}} \sim \varphi^{3 \gamma}$, but they apply also to the dilute limit $\left(\delta=\delta_{0}, \Pi=\Pi_{0}\right)$ and describe the crossover region as well. The relation $\Pi v=q_{R}^{-3} y$ for the dilute case is now extended to $\Pi v=q_{R}^{-3}\left(y+b y^{3 \gamma}\right)$, where $b$ is a known constant. Hence, the general equations given in chapter 2 may be formulated either in terms of $\Pi v$ or in terms of $y$; for mathematical reasons the variable $y$ is now easier to implement.

This generalized 'variable $q$ ' model describes both the colloid limit and the protein limit, as well as the crossover. It gives about the same cep as the 'fixed $q$ ' model based upon $\Pi=\Pi_{0}$ and $\delta=\delta_{0}$, which is not surprising because we concluded in chapter 5 that the cep is located within the dilute regime $\left(y^{*} \approx 0.35\right)$. Perhaps more surprising is the fact that outside the cep, even when $y$ is well above unity, $\Pi v(\eta)$ phase diagrams are qualitatively (and nearly quantitatively) the same as in the dilute situation (although the range for $q_{R}$ is very different). This again shows the relevance of the parameter $\Pi v$ in the thermodynamics. This equality does not hold for $\varphi(\eta)$ phase diagrams, because the dependence $\Pi v(y)$ at finite concentrations is very different from that in dilute solutions. However, also for concentrated polymer solutions and in the protein limit simple analytical equations (e.g., for critical and triple points) describe the phase diagrams quite well, with power-law exponents which are different from those in the colloid limit. In the protein limit we find a surprisingly simple result: the binodal polymer concentrations scale as $q_{R}^{1 / \gamma}$ and normalized phase diagrams $y q_{R}^{-1 / \gamma}$ versus $\eta$ become independent of the size ratio $q_{R}=R / a$.

In chapter 7 we discuss the liquid window, which is the parameter range over which a colloidal liquid is stable. In terms of the (external) polymer concentration it may be defined as the region $y^{c}<y<y^{t}$, where the superscripts refer to critical and triple points, respectively. The reference point is the cep, where $q_{R}^{*} \approx 1 / 3$ and $y^{c}=y^{t}=y^{*} \approx 0.35$; in the cep the width of the liquid window is zero. With increasing $q_{R}$ the window $y^{t}-y^{c}$ widens. In the 'fixed $q^{\prime}$ ' model $\mathrm{y}^{t}-\mathrm{y}^{c}$ increases with $q_{R}$ without bounds, but this model loses its validity for high $q_{R}$. For the new 'variable $q$ ' model the behavior close to the cep (i.e., in the colloid limit) is nearly the same, but for high $q_{R}$ (protein limit) the ratio $y^{t} / y^{c}$ becomes constant: $y^{t} / y^{c} \approx 2.2$. Then for given $q_{R}$ the liquid window spans only a factor 2.2 in the external polymer concentration $\varphi$. In terms of the internal polymer concentrations $\phi$ this ratio is somewhat larger (about 3 for the gas branch and an additional factor 2.5 for the liquid branch of the binodal), but anyhow there is only a limited range of polymer concentrations over which a colloidal liquid is stable. 
The liquid window may also be expressed in terms of the interaction strength $\varepsilon$, which is the value of the pair potential for two colloidal particles in contact. Clearly, $\varepsilon^{t}-\varepsilon^{c}$ is zero in the cep, and $\varepsilon^{t}-\varepsilon^{c}$ increases with $q_{R}$ for $q_{R}>q_{R}^{*}$. It turns out that for 'fixed $q$ ' this increase is again without bounds, whereas for 'variable $q$ ' a maximum level of about $1.6 \mathrm{kT}$ is attained. There is thus only a limited range of interaction strengths over which a liquid is stable. This balance is even more subtle for a one-component Yukawa fluid, where the liquid window $\varepsilon^{t}-\varepsilon^{c}$ is no more than (at most) $0.6 \mathrm{kT}$.

In the final chapter 8 we compare our 'variable $q$ ' theory with experiments, with simulations, and with other theories, for both good and theta solvents. In most cases we find semiquantitative agreement with experimental critical points and GL binodals. Also the agreement with simulations is quite good. Other theories sometimes deviate considerably from our prediction in the quantitative aspects, but the qualitative behavior is usually the same. A very important feature is that our $q_{R}^{1 / \gamma}$ scaling law is accurately reproduced by both simulations and other theories which are applicable to this limit.

\section{Osmotic equilibrium theory}

\subsection{System}

We consider a system where two phases with different colloid and polymer concentrations are in equilibrium with each other and with an external reservoir containing only the polymer solution (see Fig. 2.1). The phase concentrated in colloid may be solid (S) or liquid $(\mathrm{L})$, the dilute phase may be gas-like $(\mathrm{G})$ or liquid. We also use the term fluid $(F)$ to denote either the $G$ or the $L$ phase; beyond the critical point there is no difference between $\mathrm{G}$ and $\mathrm{L}$. In some cases $\mathrm{S}$ may be in equilibrium with two F phases (one liquid and the other gas); we then have a triple point where three phases coexist.

The chemical potential of the polymer in the system is determined by its volume fraction $\varphi$ in the reservoir. This volume fraction is unity in the polymer melt. In the colloid phases, the local polymer concentration in the free volume not occupied by the particles (plus the depletion layers surrounding them) is the same as the external concentration $\varphi$. However, the overall internal concentration $\phi$ is lower because the free volume is smaller than the total volume:

$\Phi_{\mathrm{x}}=\alpha_{\mathrm{x}} \varphi$

where $\mathrm{x}=\mathrm{G}$, $\mathrm{L}$, or $\mathrm{S}$ and the exclusion factor $\alpha$ is the fraction of the volume available for the polymer.

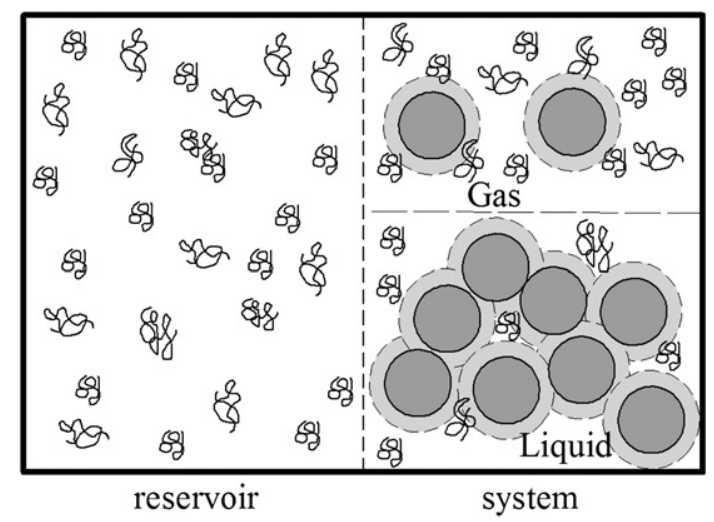

Fig. 2.1. A dilute colloidal gas in equilibrium with a concentrated colloidal liquid and with a reservoir containing only the polymer solution. The depletion layers are indicated as the grey halo around the particles. In a dilute system only pair interactions between the particles occur, in a concentrated system multiple overlap of depletion layers takes place. Throughout this paper the external (reservoir) concentration of polymer is denoted as $\varphi$, and the internal concentration in the colloid phases as $\phi$.
When the system is dilute in colloids, the depletion layers around the particles do not overlap and one expects a simple form for $\alpha$ : $\alpha=$ $\left(V-n v^{\text {eff }}\right) / V$, where $V$ is the volume of the system, $n$ the number of dispersed colloids in it, and $v^{\text {eff }}=(4 \pi / 3)(a+\delta)^{3}$ the effective volume excluded for the polymer chains by one colloid particle; here $a$ is the bare particle radius and $\delta$ is the thickness of the depletion layer around the sphere. The depletion layer is indicated by the grey halo around the particles in Fig. 2.1.

We define the dispersed colloid volume fraction $\eta$ as $\eta=n v / V$, where $v=(4 \pi / 3) a^{3}$ is the bare colloid volume. For low $\eta$ the free volume fraction $\alpha$ equals $1-\eta(1+\delta / a)^{3}$ or

$\alpha=1-\eta \zeta^{3} \quad($ small $\eta$ or $q)$

where the parameter $\zeta$ is the size ratio between particles with and without a depletion layer:

$$
\zeta=1+q \quad q=\frac{\delta}{a} .
$$

The parameter $q$ is the ratio between the depletion thickness (which is the range of the attraction) and the particle radius; it may be seen as the relative range of the attraction. The quantity $\zeta^{3}=v^{\text {eff }} / v$ is the normalized volume (per particle) which is inaccessible for the polymer chains in a dilute colloid phase.

We note that $\delta$ is the depletion thickness around a colloidal sphere, which in dilute polymer solutions and for relatively large particles is of order of (but not equal to) the radius of gyration $\mathrm{R}$ of the polymer coils. In general, the parameter $q=\delta / a$ is not equal to $q_{R}=R / a$. In Section 5.1 we show that in the colloid limit (where $\delta$ is independent of the polymer concentration) the two parameters are related through $q \approx 0.9 q_{R}^{0.9}$ (for a more precise result see Eq. (5.5)). In more concentrated solutions, where $\delta$ decreases with increasing $\varphi$, the relation between $q$ and $q_{R}$ becomes $\varphi$-dependent, see Section 6.2 Eq. (6.17). We will see that in the protein limit $\left(q_{R}>1\right)$ q becomes independent of $q_{R}$. In most of the present chapter 2 and in chapter 3 we use only the parameter $q$ (or $\zeta=1+q$ ), without specifying the relation with $q_{R}$.

For more concentrated colloid phases the depletion layers do overlap and the fraction of the volume which is excluded for the polymer is less than $\eta \zeta^{3}$ due to (multiple) overlap of depletion layers; $\alpha$ is then higher than according to the limiting form of Eq. (2.2). A more general expression for $\alpha$ is given by scaled-particle theory for the free volume [26,47], to be discussed in the next section. This theory is based upon the particle insertion method by Widom [48]. The free volume fraction follows from the work required to insert a polymer chain into a sea of colloidal spheres.

\subsection{Free volume}

In scaled-particle theory the free volume fraction $\alpha$ depends solely on the parameters $\eta$ (colloid volume fraction) and $q$ or $\zeta$ (effective size ratio), regardless of the type of phase $(\mathrm{G}, \mathrm{L}$ or $\mathrm{S})$. The expressions are more transparent when the ratio $\eta /(1-\eta)$ is used as the variable rather than $\eta$ itself. Therefore we define a concentration parameter $f$ :

$f=\frac{\eta}{1-\eta} \quad \eta=\frac{f}{1+f}$

The scaled-particle expression [26] for $\alpha$ may be written as

$\alpha=(1-\eta) \beta \quad \beta=e^{-Q}$

where $Q$ is a polynomial in $f$ :

$Q=A f+B f^{2}+C f^{3}$.

The coefficients $A, B$, and $C$ are a function of $q$ only:

$A=\zeta^{3}-1 \quad B=3 q^{2}(q+3 / 2) \quad C=3 q^{3}$. 
For small $f$ and not too large $q, \beta=1-A f$ and $\alpha=1-(A+1) f$, so Eq. (2.2) is recovered. For large $f$ and finite $q, \beta$ and $\alpha$ approach zero, as expected for concentrated colloid systems (see the concentrated phase in Fig. 2.1). It has been shown that the prediction of Eq. (2.5) for the free volume fraction agrees very well with computer simulation results [10,29], at least when $q$ is $O(1)$ or below.

A plot of $\alpha(\eta)$ for five values of $\mathrm{q}$ is given in Fig. 2.2. The initial part for small $\eta$ is linear according to $\alpha=1-\eta \zeta^{3}$ (Eq. (2.2)); this linear part extrapolates to $\alpha=0$ at $\eta=\zeta^{-3}$. For the three lowest $q$ values in Fig. 2.2 this limiting form is shown as the dashed lines; for low $q$ this form describes $\alpha(\eta)$ over a wide concentration range. For high $q, \alpha$ decays to zero at relatively small $\eta$ : the polymer is then largely excluded from the colloid phase. This effect is very pronounced for $q=5$ : the exclusion is then essentially complete for any $\eta$, even in very dilute systems. In this high- $q$ limit $Q \approx A f$ and $\alpha \approx e^{- \text {Af }}$. Hence, the exclusion limit applies when $\eta \approx f$ exceeds the value $1 / A \approx \zeta^{-3}$ by, say, a factor of 3 .

We note that in the 'fixed $q$ ' model (where $q$ is of order $q_{R}$ ) $q$ is an independent variable which may be assigned a high value. However, for 'variable $q$ ' (to be discussed in chapter 6) $q$ depends not only on $q_{R}$ but also on the polymer concentration. In that case $q$ remains below unity, even when $q_{R}$ is high (protein limit).

We will also need derivatives of $\alpha$ and, hence, of $Q$ and $\beta$ with respect to $f$. When we abbreviate $\partial^{n} X / \partial f^{n}$ as $X_{n}$, we may write

$\left\{\begin{array}{l}Q_{1}=A+2 B f+3 C f^{2} \\ Q_{2}=2 B+6 C f \\ Q_{3}=6 C\end{array}\right.$

$$
\left\{\begin{array}{l}
\beta_{1}=-\beta Q_{1} \\
\beta_{2}=-\beta Q_{2}-\beta_{1} Q_{1} \\
\beta_{3}=-\beta Q_{3}-2 \beta_{1} Q_{2}-\beta_{2} Q_{1}
\end{array} .\right.
$$

These expressions are needed to find, for given $q$, the chemical potential, the pressure, the critical point, and the critical endpoint.

\subsection{Thermodynamics}

\subsubsection{Separating hard-sphere and polymer contributions}

For a system with given numbers $n$ of colloids and $n_{p}$ of polymer chains in a given volume $V$ at temperature $T$ the characteristic thermodynamic function is the Helmholtz energy $F\left(T, V, n, n_{p}\right)$, with total differential $d F=-S d T-p d V+\mu d n+\mu_{p} d n_{p}$, where $\mu$ and $\mu_{p}$ are the chemical potentials of the colloid particles and of the polymer, respectively. For the moment we disregard the solvent, as in the original theory, but we shall correct for this in Section 2.3.3. For a semi-open

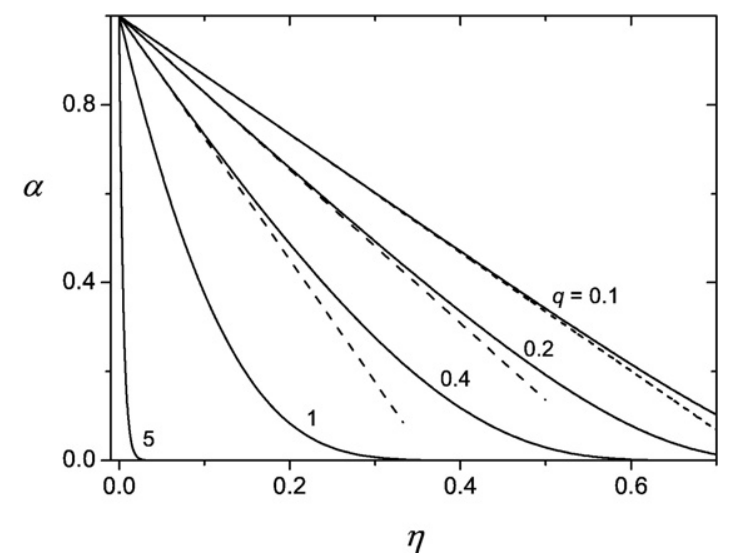

Fig. 2.2. The free volume fraction $\alpha$ as a function of the colloid concentration $\eta=f /(1+f)$ according to Eq. (2.5), for five values of the size ratio $q$. The dashed lines for $q=0.1,0.2$ and 0.4 are the dilute limit Eq. (2.2) where the depletion layers do not overlap. system like in Fig. 2.1 the variable $n_{\mathrm{p}}$ has to be replaced by the variable $\mu_{\mathrm{p}}$. The corresponding characteristic function is the grand potential $\Omega(T, V$, $n, \mu_{p}$ ), obtained by a standard Legendre transformation as

$\Omega\left(T, V, n, \mu_{p}\right)=F\left(T, V, n, n_{p}\right)-n_{p} \mu_{p}$

Its exact differential is $d \Omega=-S d T-p d V+\mu d n-n_{p} d \mu_{p}$, showing that the variable $n_{p}$ in $F$ is replaced by the variable $\mu_{p}$ in $\Omega$. The colloid chemical potential $\mu$ is found by differentiating $\Omega$ with respect to $n$. Integrating the exact differential gives $\Omega=-p V+n \mu$. Hence, when $\Omega$ is known $\mu$ and $p$ are readily obtained.

Following Lekkerkerker et al. [26] and Aarts et al. [30] we write $\Omega=\Omega^{0}+\Omega^{p}$, where $\Omega^{0}=F^{0}$ is the Helmholtz energy of a hard-sphere system without polymer and $\Omega^{p}$ is the polymer contribution. The latter is found from a build-up principle: starting from a system without polymer $\left(\varphi=0, \mu_{p}=-\infty\right)$, polymer is added to the system until the final concentration is reached. Hence,

$\Omega=\Omega^{0}+\Omega^{p} \quad \Omega^{p}=-\int_{-\infty}^{\mu_{p}} n_{p} d \mu_{p}$.

Since $\mathrm{d} \Omega^{0}=-S d T-p d V+\mu \mathrm{d} n$ and $d \Omega^{\mathrm{p}}=-n_{p} \mathrm{~d} \mu_{p}, \Omega$ in Eq. (2.11) has the same exact differential as $\Omega$ in Eq. (2.10). The assumption made in Eq. (2.11) is that the polymer does not affect the configuration of the colloids.

The polymer contribution $\Omega^{p}$ is evaluated using the free volume theory outlined in Section 2.2, whereby the polymer concentration in the colloid phases is lower than in the reservoir by a factor $\alpha$ which depends on $f$ and $q$. It is convenient to rewrite Eq. (2.11) in terms of reduced quantities:

$\omega=\frac{v}{V} \Omega \quad \eta=\frac{v}{V} n \quad \varphi=\frac{N v_{s}}{V} n_{p}$

where $v$ is the colloid volume, $v_{s}$ the segment (or solvent) volume, and $N$ the number of segments per polymer chain; the volume occupied by the segments of a chain is $N v_{s}$. Now Eq. (2.11) transforms into

$\omega=\omega^{0}+\omega^{p} \quad \omega^{p}=-\frac{v}{N v_{s}} \int_{-\infty}^{\mu_{p}} \alpha \varphi d \mu_{p}$

where in the polymer term we applied Eq. (2.1).

The chemical potential $\mu$ of the colloids and the pressure $p$ in the system are found from the standard thermodynamic relations $\mu=(\partial \Omega /$ $\partial n)_{T, V, \mu_{p}}$ and $p V=-\Omega+n \mu$ :

$\mu=\left(\frac{\partial \omega}{\partial \eta}\right)_{\varphi} \quad p v=-\omega+\eta \mu$.

Clearly, these quantities have a hard-sphere part and a polymer contribution:

$\mu=\mu^{0}+\mu^{p} \quad p v=(p v)^{0}+(p v)^{p}$.

We discuss these contributions separately in the next two sections.

\subsubsection{Hard-sphere contributions}

For a dispersion of hard spheres we have $\omega=\omega^{0}$. For a fluid phase a very accurate expression is due to Carnahan-Starling [49], and for a crystalline solid phase we use an expression based on work by Hall [50] with a numerical constant that follows from computer simulation results [51]:

$\omega^{0}=\left\{\begin{array}{lr}\eta\left[\ln \eta-1+4 f+f^{2}\right] & \text { fluid } \\ \eta\left[2.1306-3 \ln \left(\eta^{-1}-\eta_{\mathrm{cp}}^{-1}\right)\right] & \text { solid }\end{array}\right.$

where $\eta_{\mathrm{cp}}=(\pi / 6) \sqrt{2}=0.741$ is the volume fraction at close-packing. 
Applying Eq. (2.14) (with $d f=(1+f)^{2} d \eta$ ) gives the following equations for the chemical potential and the pressure:

$\mu^{0}=\left\{\begin{array}{lr}\ln \eta+8 f+7 f^{2}+2 f^{3} & \text { fluid } \\ 2.1306+3 /\left(\eta^{-1}-\eta_{\mathrm{cp}}^{-1}\right)-3 \ln \left(\eta^{-1}-\eta_{\mathrm{cp}}^{-1}\right) & \text { solid }\end{array}\right.$

$(p v)^{0}= \begin{cases}\eta+4 f^{2}+2 f^{3} & \text { fluid } \\ 3 /\left(\eta^{-1}-\eta_{\mathrm{cp}}^{-1}\right)=3 /\left(f^{-1}-f_{\mathrm{cp}}^{-1}\right) & \text { solid }\end{cases}$

where $f_{\mathrm{cp}}=\eta_{\mathrm{cp}} /\left(1-\eta_{\mathrm{cp}}\right)=2.853$. Both $\mu_{\mathrm{s}}^{0}$ and $(p v)_{s}^{0}$ for a solid diverge at close-packing: $\eta=\eta_{\mathrm{cp}}=0.741$ or $f=f_{\mathrm{cp}}=2.853$.

For calculating GL critical points and the critical endpoint we will also need the first and second derivatives of Eq. (2.18a) with respect to $f$ :

$\left\{\begin{array}{l}(p v)_{1}^{0}=(1+f)^{-2}+8 f+6 f^{2} \\ (p v)_{2}^{0}=-2(1+f)^{-3}+8+12 f\end{array}\right.$

Note that a numerical subscript 1 or 2 denotes the first or second derivative with respect to $f$.

Fig. 2.3 gives plots of $\mu_{f}^{0}$ and $\mu_{s}^{0}$ (dashed curves) and of $(p v)_{f}^{0}$ and $(p v)_{s}^{0}$ (solid) as a function of $f$. There is a well-known fluid-solid phase coexistence at $\eta_{f}^{0}=0.494$ and $\eta_{s}^{0}=0.545$ according to computer simulations [52-54]. Essentially the same result follows from the above equations with the condition $\mu_{f}^{0}=\mu_{s}^{0}$ and $p_{f}^{0}=p_{s}^{0}: f_{f}^{0}=0.970$ $\left(\eta_{f}^{0}=0.492\right)$ and $f_{s}^{0}=1.185\left(\eta_{s}^{0}=0.542\right)$. This coexistence condition is indicated by the rectangle in Fig. 2.3, with $\mu_{0}^{0}=15.463$ and $(p v)_{0}^{0}=6.081$ at coexistence. These numbers may be calculated by writing $1 / f_{s}=1 / f_{\mathrm{cp}}+3 /(p v)^{0}$ from Eq. (2.18a). Substituting $p^{0}=p_{f}^{0}\left(f_{f}\right)$ from Eq. (2.18) gives $f_{s}$ as a function of $f_{f}$. Inserting this relation into $\mu_{f}^{0}$ $\left(f_{f}\right)=\mu_{s}^{0}\left(f_{s}\right)$ leads to an implicit equation in $f_{f}$ which is easily solved numerically. This procedure is entirely equivalent to applying the common tangent construction to $\omega_{f}^{0}(f)$ and $\omega_{s}^{0}(f)$.

\subsubsection{Polymer contribution}

We rewrite the polymer contribution of Eq. (2.13b) in terms of the osmotic pressure $\Pi$ of the external reservoir, taking the solvent into account as a component. When the solvent is treated as background, the solvent molecules do not occupy volume, and polymer chains can be added to the system without affecting the solvent. We treat the solvent molecules as entities occupying the same volume $v_{s}$ as polymer segments. The implication is that upon adding one polymer chain to the system $N$ solvent molecules have to leave. Consequently, we replace $\mu_{p}$ in Eq. (2.13b) by an exchange chemical potential $\mu^{\mathrm{e}}$, defined by $\mu^{e} \equiv \mu_{p}-N \mu_{s}$, where $\mu_{s}$ is the chemical potential of the solvent.

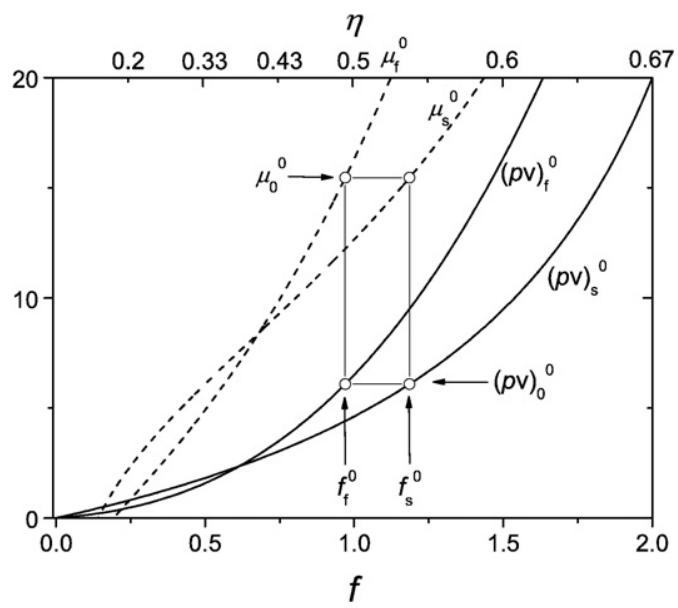

Fig. 2.3. The hard-sphere chemical potentials $\mu_{f}^{0}$ (fluid) and $\mu_{s}^{0}$ (solid) and the corresponding $(p v)_{f}^{0}$ and $(p v)_{s}^{0}$ as a function of $f$. The rectangle indicates the FS coexistence where $\mu=\mu_{0}^{0}=15.463$ and $p v=(p v)_{0}^{0}=6.081$, at concentrations $f_{f}^{0}=0.970$ and $f_{s}^{0}=1.185$ (or $\eta_{f}^{0}=0.492, \eta_{s}^{0}=0.542$ ).

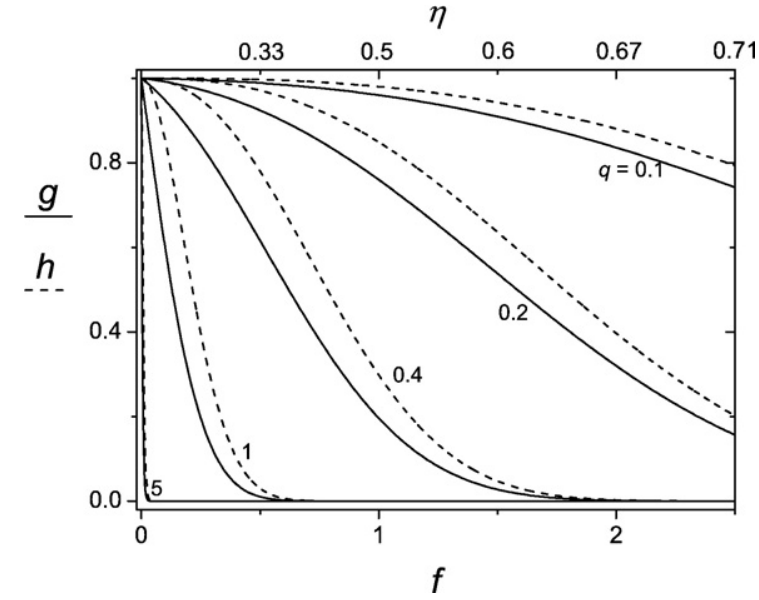

Fig. 2.4. The functions $g$ (Eq. (2.21b), solid curves) and $h$ (Eq. (2.22b), dashed) as a function of $f$, for five values of $q$.

In Eq. (2.13b) we then need $\varphi d \mu^{e}=\varphi\left(d \mu_{p}-N d \mu_{s}\right)$. From the GibbsDuhem rule we have $n_{p} d \mu_{p}+n_{s} d \mu_{s}=0$, where $n_{s}$ is the number of solvent molecules. In terms of $\varphi$ this gives $\varphi \mathrm{d} \mu_{p}+(1-\varphi) N d \mu_{s}=\varphi d \mu^{e}+$ $N d \mu_{s}=0$. Since $\mu_{s}=-\Pi v_{s}$, we obtain $\varphi d \mu^{e}=N v_{s} d \Pi$, so Eq. (2.13) can be written in the simple form

$\omega^{p}=-\int_{0}^{\Pi v} \alpha d \Pi v$

We see that the product $\Pi v$ is a natural parameter in the thermodynamic description. It is the osmotic work to insert a particle (without depletion layer) into the polymer solution. Eq. (2.20) is general, but for applying it the relation between $\alpha$ and $\Pi v$ is needed. We recall that $\alpha$ is a function of $f$ and $q=\delta / a$. In the colloid limit, where $\delta$ is constant (independent of $\varphi$ or $\Pi$ ), Eq. (2.20) simplifies to $\omega^{p}=$ $-\alpha \Pi v$ (see also Eq. (2.29)). In the general case where $\delta$ and $q$ decrease with increasing $\Pi v$, the integration of Eq. (2.20) cannot be avoided; one then has to specify the relation between $q$ and $\Pi v$.

Applying again Eq. (2.14) we obtain the polymer contributions to $\mu$ and $p v$ :

$\mu^{p}=\int_{0}^{\Pi v} \zeta^{3} g d \Pi v \quad \quad \zeta^{3} g=\beta-(1+f) \beta_{1}$

$(p v)^{p}=\int_{0}^{\Pi v} h d \Pi v \quad h=\beta-f \beta_{1}$.

The functions $g$ and $h$ are defined in terms of $\beta$ Eq. (2.5) and its first derivative Eq. (2.9). For $f=0, \beta=1$ and $\beta_{1}=-A$, so $h=1$ and $\zeta^{3} g=1+$ $A=\zeta^{3}$ or $g=1$. For large $f, \beta=\beta_{1}=0$ and $g=h=0$. Plots of the functions $g$ and $h$ as a function of $f$ are given in Fig. 2.4. Both functions decay in roughly the same way from unity in dilute systems to zero in concentrated colloidal dispersions. This decay is faster as $q$ increases. The function $g$ (solid curves) for intermediate $f$ is somewhat smaller than the function $h$ (dashed). For large $q$ (see the curve for $q=5$ in Fig. 2.4) $g$ and $h$ reach the limits $g=e^{-A f}$ and $h=(1+A f) e^{-A f}$ so $g$ and $h$ are essentially zero for $f>A^{-1}$ or $f>\zeta^{-3}$. Then we have the exclusion limit $\mu \approx \mu^{0}$ and $p \approx p^{0}$ for nearly any colloid concentration.

For the calculation of critical points we need the first and second derivatives of Eq. (2.22) with respect to $f$. These derivatives may be written in terms of the second and third derivatives of $\beta$ (defined in Eq. (2.9)):

$$
\left\{\begin{array}{l}
(p v)_{1}^{p}=-f \int_{0}^{\Pi v} \beta_{2} d \Pi v \\
(p v)_{2}^{p}=-\int_{0}^{\Pi v}\left(\beta_{2}+f \beta_{3}\right) d \Pi v
\end{array}\right.
$$




\subsection{Binodals, critical and triple points, and critical endpoint}

Binodal points are obtained from equal chemical potentials (Eq. (2.17) plus (2.21)) and equal pressures (Eq. (2.18) plus (2.22)) in the two coexisting phases:

$$
\begin{array}{lll}
\text { FS } & \mu_{f}=\mu_{s} & (p v)_{f}=(p v)_{s} \\
\text { GL } & \mu_{g}=\mu_{l} & (p v)_{g}=(p v)_{l} .
\end{array}
$$

Here $\mu_{g}=\mu_{f}\left(f_{g}\right)$ and $\mu_{l}=\mu_{f}\left(f_{l}\right)$, and similarly for $p v$.

For the GL critical points the first and second derivatives of the pressure (or the chemical potential) with respect to $f$ are zero:

ср $\quad(p v)_{1}=(p v)_{2}=0$

The two contributions to these derivatives are given in Eqs. (2.19) and (2.23), respectively.

Triple points follow from equal chemical potentials and pressures in three phases:

tp $\quad \mu_{g}=\mu_{l}=\mu_{s} \quad(p v)_{g}=(p v)_{l}=(p v)_{s}$

Finally, for the cep we have critical conditions supplemented with equal chemical potential and pressure in fluid and solid phases:

сер

$$
(p v)_{1}=(p v)_{2}=0 \quad \mu_{f}=\mu_{s} \quad(p v)_{f}=(p v)_{s} .
$$

In order to apply these expressions, we have to specify how $q$ in the integrals of the polymer contributions depends on $q_{R}$ and $\Pi v$. We postpone the general formulation of this problem to chapter 6 , but we give one example of the type of dependence. It can be shown that in a semidilute good solvent $\Pi v \sim q_{R}^{-3} y^{2.31}$ (Eq. (6.12)) and $q \sim q_{R}^{0.88} y^{-0.68}$ (Eq. (6.17)), where $y=\varphi / \varphi_{\text {ov }}$. Combining these two relations we find $q \sim(\Pi v)^{-0.37}$, independent of $R$ or $q_{R}$, as expected for semidilute solutions. Inserting these relations (with the appropriate numerical constants) into the integrals for $\mu^{\mathrm{p}}$ and $(p v)^{p}$, we could from Eq. (2.24) or (2.25) solve the two equations in the two unknown compositions ( $f_{f}$ and $f_{s}$ for FS, $f_{g}$ and $f_{l}$ for GL) at given $q_{R}$ and $\Pi v$ to find the binodals. Similarly, we could from Eq. (2.26) (again two equations in two unknowns) find $\Pi v$ and $f$ at given $q_{R}$ to obtain the critical points.

For finding triple points, we have to solve the four equations of Eq. (2.27) for $\Pi v$ and three compositions, again at given $q_{R}$. Finally, the cep follows from solving the four equations of Eq. (2.28) for $q_{R}^{*},(\Pi v)^{*}$, $f^{*}$, and $f_{s}^{*}$.

The procedure outlined above is inaccurate since the scaling relation $q \sim(\Pi v)^{-0.37}$ breaks down in the dilute regime $(y<1)$. It is possible (see Section 6.2) to find relations $\Pi v\left(q_{R}, y\right)$ and $q\left(q_{R}, y\right)$ which are valid over the entire concentration range. However, now the relation $q\left(q_{R}, \Pi v\right)$ is implicit, which is computationally unhandy. It is then easier to use $y$ instead of $\Pi v$ as the integration variable, replacing $d \Pi v$ in the integrals by $(\partial \Pi v / \partial y) d y$. Details of the necessary expressions are given in Section 6.2.

\subsection{Chemical potential and pressure for 'fixed q'}

When $q$ does not depend on $\Pi v$, which is the case in the colloid limit, the integrand in Eqs. (2.20)-(2.22) is a constant and may be taken out of the integral. Now $\omega, \mu$, and $p v$ reduce to

$$
\begin{aligned}
& \omega=\omega^{0}-\alpha \Pi v \\
& \mu=\mu^{0}+\Pi v \zeta^{3} g \\
& p v=(p v)^{0}+\Pi v h .
\end{aligned}
$$

Similarly, Eq. (2.23) may be simplified and the first and second derivatives of $p v$ are given by

$\left\{\begin{array}{l}(p v)_{1}=(p v)_{1}^{0}-f \beta_{2} \Pi v \\ (p v)_{2}=(p v)_{2}^{0}-\left(\beta_{2}+f \beta_{3}\right) \Pi v\end{array}\right.$.

Now all Eqs. (2.24)-(2.28) for calculating binodals, critical and triple points, and the cep can be rewritten such as to give one equation in one unknown. For example, Eq. (2.24) for FS binodals reduces to $\mu_{f}^{0}+$ $\Pi v \zeta^{3} g_{f}=\mu_{s}^{0}+\Pi v \zeta^{3} g_{s}$ and $(p v)_{f}^{0}+\Pi v h_{f}=(p v)_{s}^{0}+\Pi v h_{s}$, from which $\Pi v$ may be eliminated. The result (which is shown in Eq. (3.1)) gives a direct relation between $f_{f}$ and $f_{s}$. So when a value for $f_{s}$ is chosen, $f_{f}$ follows immediately from solving one equation in one unknown.

In the next chapter we calculate full phase diagrams for 'fixed $q$ '. First we illustrate some features of the simple Eqs. (2.30)-(2.31) for $\mu$ and $p v$. An example of $\mu(f)$ and $p v(f)$ for a fluid phase $\left(\mu^{0}=\mu_{f}^{0},(p v)^{0}=\right.$ $\left.(p v)_{f}^{0}\right)$ is given in Fig. 2.5, for $q=0.6(\zeta=1.6)$ and three values of $\Pi$ : $\Pi_{1} v=2, \Pi_{2} v=2.259$ and $\Pi_{3} v=2.738$. The value of $\Pi_{2}$ corresponds to $\Pi^{c}$, the osmotic pressure at the critical point.

For a low polymer concentration (say, $\Pi=\Pi_{1}$ ), both $\mu$ and $p v$ increase monotonically with the colloid concentration. Hence, there is no possibility for phase separation into a dilute $G$ phase and a concentrated $\mathrm{L}$ phase, since such a demixing requires equal $\mu$ and $\mathrm{p}$ in both phases. For a high polymer concentration $\left(\Pi=\Pi_{3}\right)$, both $\mu$ and $p$ show a Van der Waals loop. In this particular example we have equal $\mu$ 's $(\mu=7.878)$ at three points: $f_{g}=0.052, f_{l}=0.5$ and a metastable point somewhere in between. For this $\Pi$ we have equal $p$ 's $(p v=2.779)$ at
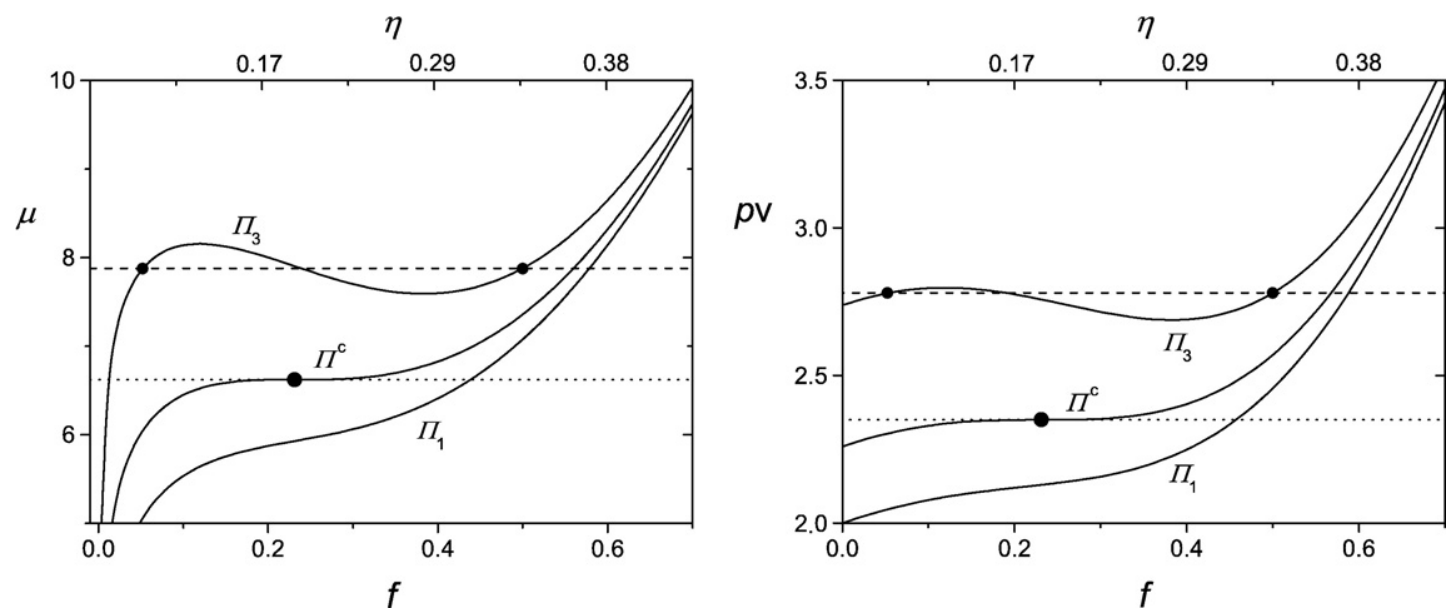

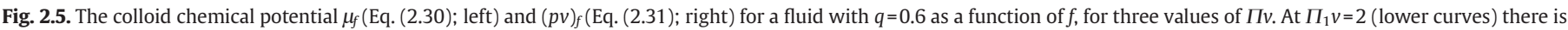

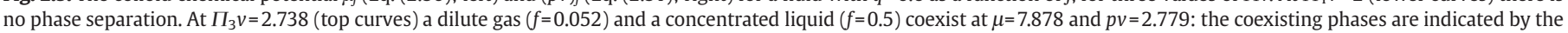
small dots. At $\Pi^{c} v=2.259$ (middle) both $\mu$ and $p$ have an inflection point where $\mu^{\prime}=\mu^{\prime \prime}=p^{\prime}=p^{\prime \prime}=0$ : this is the critical point, indicated by the large dot. 


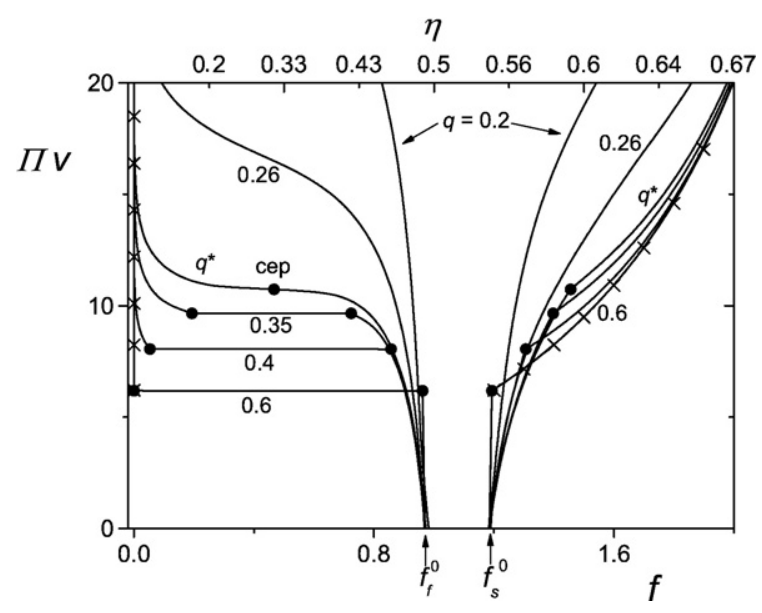

Fig. 3.1. FS binodals for $q=0.2,0.26,0.3275\left(q^{*}\right), 0.35,0.4$, and 0.6 . The circles indicate the triple points for the four highest $q$ values; the triple point for $q=q^{*}$ is the cep. The crosses indicate the high- $q$ (exclusion) limit of the binodals.

exactly the same colloid concentrations, so these two points $f_{g}=0.052$ and $f_{l}=0.5$ lie on the binodal.

In between, for $\Pi=\Pi^{c}$, there is an inflection point with zero slope in both curves at $f=f^{c}$. In this point the first and second derivatives of $\mu$ and $p$ are zero. From these conditions, the location of the critical point, which is the lowest point of the binodal, may be derived, see Eq. (2.26); for $q=0.6$ the result is $(\Pi v)^{c}=2.259$ and $f^{c}=0.231$.

\section{Phase diagrams for 'fixed $q$ ' in terms of $\Pi v$}

In this chapter we assume that $\delta$ and $q$ are independent of the polymer concentration (so Eqs. (2.29)-(2.32) apply) and we use only the product $\Pi v$ to characterize the polymer solution. There is no need to specify how $\Pi$ depends on the polymer concentration $\varphi$. The conversion towards $\varphi$ is discussed in chapter 5 .

\subsection{Calculation of the phase diagram}

\subsubsection{FS binodals}

Substituting Eqs. (2.30)-(2.31) into Eq. (2.24) gives $\mu_{f}^{0}+\Pi v \zeta^{3} g_{f}=\mu_{s}^{0}+$ $\Pi v \zeta^{3} g_{s}$ for the chemical potentials and $(p v)_{f}^{0}+\Pi v h_{f}=(p v)_{s}^{0}+\Pi v h_{s}$ for the pressures. After eliminating $\Pi v$ from these two relations, we obtain:

$\Pi v=\frac{1}{\zeta^{3}} \frac{\mu_{s}^{0}-\mu_{f}^{0}}{g_{f}-g_{s}}=\frac{(p v)_{s}^{0}-(p v)_{f}^{0}}{h_{f}-h_{s}}$.

Here $\mu_{f}^{0}$ (Eq. (2.17a)) and ( $\left.p v\right)_{f}^{0}$ (Eq. (2.18a)) depend only on $f_{f}$, and $\mu_{s}^{0}$ (Eq. (2.17b)) and ( $\left.p v\right)_{s}^{0}$ (Eq. (2.18b)) only on $f_{s}$. For a given value of $q$, the same applies to the $g$ 's and $h$ 's, respectively. Hence, the second and third parts of Eq. (3.1) give a unique relation $f_{f}\left(f_{s}\right)$ : when a value for $f_{s}$ is chosen, the corresponding value of $f_{f}$ is found by solving the second equality of Eq. (3.1), and $\Pi v$ follows immediately from the first. Fig. 3.1 gives six FS binodals, for $q=0.2,0.26,0.3275,0.35,0.4$, and 0.6 . The value $q^{*}=0.3275$ corresponds to the critical endpoint, see Eq. (3.7) which gives the precise coordinates of the cep.

All $F$ branches in Fig. 3.1 start at $f_{f}^{0}=0.970$ and all $S$ branches at $f_{s}^{0}=1.185$ for $\Pi v=0$; these are the hard-sphere coexistence concentrations discussed in Section 2.3.2, see Fig. 2.3. For thin depletion layers $(q=0.2)$, there is not much difference with hard spheres without polymer. Also the pressure of the system does not deviate much from that of hard spheres ( $p v=6.08)$ because $h$ in Eq. $(2.31)$ is small. The composition of the fluid phase becomes slightly more dilute and that of the solid phase more concentrated as $\Pi v$ increases: the miscibility gap widens somewhat as the polymer concentration goes up. For slightly thicker depletion layers $(q=0.26)$ this widening is more pronounced at high $\Pi v$.
For $q=q^{*}=0.3275$, the F-branch is nearly horizontal over a wide range of colloid concentrations and it has an inflection point with zero slope of the curve. This inflection point (top circle left) is the fluid part of the cep, situated at $\Pi v=10.73$ and $f=0.467$ (see Eq. (3.7)). The solid branch has a discontinuity in slope at the same $\Pi v$ and $f=1.457$ (top circle right), which is the solid part of the cep.

When $q>q^{*}$, the fluid branch becomes discontinuous (there is a jump from the $\mathrm{L}$ branch to the $\mathrm{G}$ branch at a certain $\Pi v$ ), and the discontinuity in slope of the solid branch (at the same $\Pi v$ ) becomes more pronounced. For example, for $q=0.35$ this discontinuity occurs at $\Pi v=9.66$ and $f$-values $0.193(G), 0.724(\mathrm{~L})$, and $1.398(\mathrm{~S})$. This is the triple point for this $q$ value, where three phases coexist. For $q=0.4$ the triple point coordinates are $\Pi v=8.05$ and $f=0.054(\mathrm{G}), 0.857(\mathrm{~L})$, and $1.306(\mathrm{~S})$, and for $q=0.6$ these values are $\Pi v=6.18$ and $f=7.10^{-5}(\mathrm{G})$, $0.962(\mathrm{~L})$, and $1.193(\mathrm{~S})$. The coordinates of these three triple points are also indicated as closed circles in Fig. 3.1.

For still higher $q$ the FS binodals and the triple point coordinates are nearly the same as for $q=0.6$. This is because for very thick depletion layers the polymer is nearly fully excluded from the condensed phases. Hence, in Eq. (2.31) the polymer contribution may be neglected $(h=0)$, so $p v=(p v)_{s}^{0}$ in the solid phase. In the (very) dilute gas phase the polymer contribution dominates: $p v=\Pi v$ since $h=1$ and the colloid contribution to the pressure is negligible. Since the two coexisting phases should have the same pressure $\Pi v=(p v)_{s}^{0}$, with $(p v)_{s}^{0}$ the hard-sphere contribution given by Eq. (2.18b). The binodal for $q=\infty$ is indicated by the crosses in Fig. 3.1. The gas branch coincides with the ordinate axis, the solid branch follows $\Pi v=(p v)_{s}^{0}$, which is the part $f>f_{s}^{0}$ of $(p v)_{s}^{0}$ in Fig. 2.3. The triple-point value of $\Pi v$ in this limit is where the solid branch intersects the vertical line at $f=f_{s}^{0}=1.185$, and is given by $(p v)_{0}^{0}=6.081$.

\subsubsection{GL binodals}

Analogously to Eq. (3.1) the coexistence concentrations $f_{g}$ and $f_{l}$ follow from the relations $\mu_{g}^{0}+\Pi v \zeta^{3} g_{g}=\mu_{l}^{0}+\Pi v \zeta^{3} g_{l}$ and $(p v)_{g}^{0}+\Pi v h_{g}=$ $(p v)_{l}^{0}+\Pi v h_{l}$, or

$\Pi v=\frac{1}{\zeta^{3}} \frac{\mu_{l}^{0}-\mu_{g}^{0}}{g_{g}-g_{l}}=\frac{(p v)_{l}^{0}-(p v)_{g}^{0}}{h_{g}-h_{l}}$

where $\mu_{l}^{0}=\mu_{f}^{0}\left(f_{l}\right)$ and $\mu_{g}^{0}=\mu_{f}^{0}\left(f_{g}\right)$, with $\mu_{f}^{0}$ given by Eq. (2.17a), and similarly for the $(p v)^{0}$ s and Eq. (2.18a). In Eq. (3.2) the same functional form for $\mu_{f}^{0}$ and $(p v)_{f}^{0}$ for the coexisting phases applies, whereas in Eq. (3.1) these forms are different.

Fig. 3.2 gives some examples of GL binodals, for $q=0.3275\left(=q^{*}\right)$, $0.35,0.4$, and 0.6 . The GL binodal for $q=q^{*}$ is a single point (point

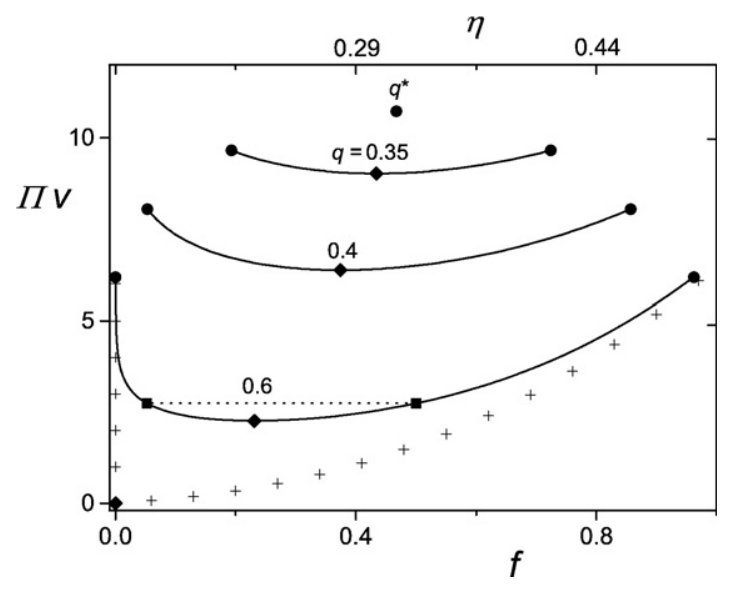

Fig. 3.2. GL binodals for $q=0.3275$ ( $q^{*}$, point binodal), $0.35,0.4$, and 0.6 . The circles for the triple points (and the cep) are the same as in Fig. 3.1, the diamonds are the critical points. The plusses indicate the high- $q$ exclusion limit of the GL binodal. The squares are the two binodal points from Fig. 2.5. 
binodal), and for higher $q$ the binodal spans a wider region, both in terms of the colloid concentration range and in terms of $\Pi v$. The endpoints left and right of each binodal are the $G$ and $L$ parts of the triple point (circles; these are the same as in Fig. 3.1), the minimum is the critical point (diamonds); it follows from Eq. (2.26) in combination with Eq. (2.32). The coordinates of the three critical points are $\Pi v=9.02, f=0.434$ for $q=0.35, \Pi v=6.38, f=0.374$ for $q=0.4$, and $\Pi v=2.26, f=0.231$ for $q=0.6$. For comparison, the two binodal points for $q=0.6$ from Fig. 2.5 are indicated as the two squares in Fig. 3.2.

The plusses in Fig. 3.2 give the high- $q$ limit for the GL binodal, obtained in the same way as the crosses in Fig. 3.1 by assuming exclusion of the polymer from the liquid phase. Hence, the $G$ branch coincides with the ordinate axis, and the L branch is $\Pi v=(p v)_{f}^{0}$, given by Eq. (2.18a). In this limit the critical point is at $\Pi v=f=0$. This is a typical 'fixed $q$ ' result; we will see later that when $q$ is allowed to vary with polymer concentration a non-zero value is found for high $q_{R}=R / a$.

\subsubsection{Triple points}

In a triple point we have equal p's and $\mu$ 's at three compositions. We may write down three equations of the type of Eq. (3.1) or (3.2), for the GS, GL, and LS coexistence, respectively. Obviously, we need only two of those. When we choose GL and LS we have

$\Pi_{g l} v=\frac{1}{\zeta^{3}} \frac{\mu_{l}^{0}-\mu_{g}^{0}}{g_{g}-g_{l}}=\frac{(p v)_{l}^{0}-(p v)_{g}^{0}}{h_{g}-h_{l}} \quad \Pi_{l s} v=\frac{1}{\zeta^{3}} \frac{\mu_{s}^{0}-\mu_{l}^{0}}{g_{l}-g_{s}}=\frac{(p v)_{s}^{0}-(p v)_{l}^{0}}{h_{l}-h_{s}}$.

where $\mu_{g}^{0}=\mu_{f}^{0}\left(f_{g}\right), \mu_{l}^{0}=\mu_{f}^{0}\left(f_{l}\right), \mu_{s}^{0}=\mu_{s}^{0}\left(f_{s}\right)$, and similarly for the functions $(p v)^{0}$. With $\Pi_{g l}=\Pi_{l s}$ Eq. (3.3) constitutes a set of four equations from which the four coordinates $f_{g}^{t}, f_{l}^{t}, f_{s}^{t}$ and $(\Pi v)^{t}$ may be found. The problem can be reduced to one equation in one unknown. For given $q$ we start with an initial estimate for $f_{l}$. Then the second part of Eq. (3.3b) gives the corresponding $f_{s}\left(f_{l}\right)$, and similarly $f_{g}\left(f_{l}\right)$ is found from Eq. (3.3a). Then also $\Pi_{g l} v$ and $\Pi_{l s} v$ are available. Equating them gives one implicit equation with $f_{l}$ as the only unknown.

Fig. 3.3 (circles) shows triple points for $17 q$ values as indicated in the legend. When the circles are connected by a continuous curve, we obtain the triple curve, which has three branches. The top is the cep at $(\Pi v)^{*}=10.73, f^{*}=0.467, f_{s}^{*}=1.457$. With increasing $q,(\Pi v)^{t}$ decreases but for high $q$ a lower limit is reached: all the triple points for $q>0.6$ essentially coincide (bottom) at the values $(\Pi v)^{t}=(p v)_{0}{ }^{0}=6.081, f_{g}^{t}$ close to zero, $f_{l}^{t}=f_{f}^{0}=0.970$, and $f_{s}^{t}=f_{s}^{0}=1.185$. The continuous curves

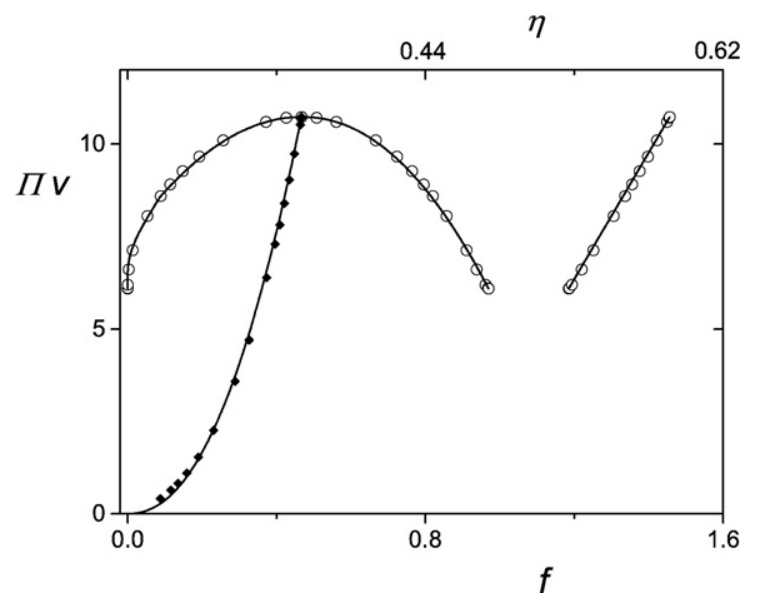

Fig. 3.3. Triple points (circles) and critical points (diamonds) for a wide range of $q: q=q^{*}=0.3275$ (cep, top), 0.328, 0.33, 0.34, 0.35, 0.36, 0.37, 0.38, 0.4, 0.45, 0.5, 0.6, 0.7, $0.8,0.9,1$, and 1.2 (this is the standard set from Table 5.1). The curves are analytical approximations: Eq. (3.18c) for the critical curve, Eq. (3.23) for the gas branch of the triple curve, and Eq. (3.21) for the liquid branch. The curve for the solid branch of the triple point is a simple straight line from $(\Pi v)^{*}, f_{s}^{*}$ to $(p v)_{0}^{0}, f_{s}^{0}$. for the triple points in Fig. 3.3 are analytical approximations, which will be discussed in Section 3.2.

\subsubsection{Critical points}

For the critical point at given $\mathrm{q}$ we have the two equations $d p /$ $d f=d^{2} p / d f^{2}=0\left(\right.$ or $\left.d \mu / d f=d^{2} \mu / d f^{2}=0\right)$ from which the two unknowns $f^{c}$ and $(\Pi v)^{c}$ follow. Analytical forms of the derivatives of $p v$ are given in Eq. (2.32), with Eq. (2.19) for the HS part. Hence, $(p v)_{1}^{0}-f \beta_{2} \Pi v=(p v)_{2}^{0}-$ $\left(\beta_{2}+f \beta_{3}\right) \Pi v=0$. Eliminating $\Pi v$, we have

$(\Pi v)^{c}=\frac{(p v)_{1}^{0}}{f \beta_{2}}=\frac{(p v)_{2}^{0}}{\beta_{2}+f \beta_{3}}$.

The second and third parts may be rewritten as

$\frac{1}{f}+\frac{\beta_{3}}{\beta_{2}}-\frac{(p v)_{2}^{0}}{(p v)_{1}^{0}}=0$

Since $\beta_{2}$ and $\beta_{3}$ (Eq. (2.9)) are only a function of $q$ and $f$, Eq. (3.5) for given $q$ constitutes an implicit equation in one unknown $f$; its solution gives $f^{c}$. Then $\Pi v$ follows from

$(\Pi v)^{c}=\left.\frac{(p v)_{1}^{0}}{f \beta_{2}}\right|_{f=f_{c}}$.

Fig. 3.3 (diamonds) gives the critical points for the same $17 q$ values as for the triple points. The solid curve (the critical curve) is a simple power-law approximation $(\Pi v)^{c} \sim\left(f^{c}\right)^{2.2}$, discussed further in Section 3.2.

\subsubsection{Critical endpoint}

For the critical endpoint (cep) we have also these Eqs. (3.5) and (3.6), supplemented with the two of Eq. (3.3b). From an initial estimate of $q^{*}$ we first calculate $(\Pi v)^{*}$ and $f^{*}$ from Eqs. (3.5) and (3.6). Now $f_{s}^{*}$ follows from the second part of Eq. (3.3b), and the first part gives $\Pi_{f s} v$, which should be equal to $(\Pi v)^{*}$. This fixes $q^{*}$ and provides then also the other three coordinates. The result, which was already used in Figs. 3.1-3.3 is

$$
\begin{array}{ll}
q^{*}=0.3275 & f^{*}=0.4673\left(\eta^{*}=0.3185\right) \\
f_{s}^{*}=1.4565\left(\eta_{s}^{*}=0.5929\right) & (\Pi v)^{*}=10.7293 .
\end{array}
$$

\subsection{Analytical approximations}

\subsubsection{The exclusion limit}

In Figs. (3.1) and (3.2) several examples may be found where a very dilute colloidal gas is in equilibrium with a concentrated fluid or solid phase, from which the polymer is nearly fully excluded. In this socalled exclusion limit we may neglect the polymer contribution to the pressure (and chemical potential) in the concentrated phases, setting $h_{l}$ and $h_{s}$ (and $g_{l}$ and $g_{s}$ ) zero. Then Eq. (2.31) reduces to $p v=(p v)^{0}$ in the condensed phases. In the very dilute colloidal gas the polymer concentration is essentially the same as in the reservoir, which implies $g_{g}=h_{g}=1$. Hence $p v=\Pi v$ in the dilute gas phase: the contribution of the colloids to the pressure is negligible. Because the gas and condensed phases should have the same pressure, we have for the liquid and solid branches of the binodal

$\Pi v= \begin{cases}(p v)_{l}^{0} & \text { liquid } \\ (p v)_{s}^{0} & \text { solid }\end{cases}$

Hence, in this limit the liquid and solid branches of the binodal are the same as the solid curves in Fig. 2.3 below $f_{f}^{0}$ and above $f_{s}^{0}$, respectively, as already shown by the crosses in the $S$ branch of Fig. 3.1, and by the plusses for the L branch in Fig. 3.2.

In Figs. (3.1) and (3.2) the gas branch in the exclusion limit was assumed to coincide with the ordinate axis. We can find a better approximation. In Eq. (3.1) we change the subscript into $_{g}$ to describe 
a GS equilibrium, and we neglect $g_{s}$ and $h_{s}$ with respect to $g_{g}=h_{g}=1$ and $p_{g}^{0}$ with respect to $p_{s}^{0}$. A similar procedure is followed in Eq. (3.2) for the GL equilibrium. We then can write these equations as

$\Pi v=\left\{\begin{array}{ll}\zeta^{-3}\left(\mu_{l}^{0}-\mu_{g}^{0}\right)=(p v)_{l}^{0} & \text { liquid } \\ \zeta^{-3}\left(\mu_{s}^{0}-\mu_{g}^{0}\right)=(p v)_{s}^{0} & \text { solid }\end{array}\right.$.

Next we take for $\mu_{g}^{0}$ the ideal contribution $\mu_{g}^{0}=\ln \eta_{g}$. Then the gas branches are given by

$\ln \eta_{g}= \begin{cases}\mu_{l}^{0}-\zeta^{3}(p v)_{l}^{0} & \text { liquid } \\ \mu_{s}^{0}-\zeta^{3}(p v)_{s}^{0} & \text { solid }\end{cases}$

For a solid a direct relation between $\mu_{s}^{0}$ and $(p v)_{s}^{0}$ is obtained by substituting $1 / f=1 / f_{\mathrm{cp}}+3 /(p v)_{s}^{0}$ from Eq. (2.18b) into Eq. (2.17b):

$\mu_{s}^{0}=1.835+1.35(p v)_{s}^{0}+3 \ln (p v)_{s}^{0}$

where the first constant equals $2.1306+3(1-\ln 3)$ and the second is $1 / \eta_{\mathrm{cp}}$.

For a liquid there is also a one-to-one relation between $\mu_{l}^{0}$ and $(p v)_{l}^{0}$, but this relation is implicit since $f$ cannot be found explicitly from Eq. (2.17a) or (2.18a). We can derive an explicit approximation by assuming power-law behavior around the hard-sphere coexistence values $\mu_{0}^{0}=15.463$ and $(p v)_{0}^{0}=6.081$ :

$\mu_{l}^{0} / \mu_{0}^{0}=\left(p_{l}^{0} / p_{0}^{0}\right)^{\tau} \quad \tau=\left.\frac{d \ln \mu_{l}^{0} / d f}{d \ln (p v)_{l}^{0} / d f}\right|_{f=f_{f}^{0}}=0.799$

The exponent $\tau$ is found from analytical or numerical differentiation of Eqs. (2.17a), (2.18a). Eq. (3.12) turns out to be very accurate in the range $2<(p v)_{l}^{0}<30$, but breaks down for small $(p v)_{l}^{0}$ where the logarithmic term in Eq. (2.17) (diverging for $f=0,(p v)_{l}^{0}=0$ ) becomes the leading term. In a very dilute system $\mu^{0}=\ln \eta$ and $(p v)_{l}^{0}=\eta$, so $\mu^{0}=\ln (p v)_{l}^{0}$. We employ the following empirical correction to Eq. (3.12):

$\mu_{l}^{0}=3.65\left((p v)_{l}^{0}\right)^{0.8}+\Theta\left[1-(p v)_{l}^{0}\right] \ln (p v)_{l}^{0}$

where the Heaviside function $\Theta(x)$ is unity for positive $x,(p v)_{l}^{0}<1$, and zero for negative $x,(p v)_{l}^{0}>1$. The prefactor 3.65 is $\mu_{0}^{0}\left((p v)_{0}^{0}\right)^{-0.8}$.

Fig. 3.4 gives a plot of $\mu_{l}^{0}$ as a function of $(p v)_{l}^{0}$ and of $\mu_{s}^{0}$ as a function of $(p v)_{s}^{0}$. The symbols are the results according to Eqs. (2.17) and (2.18), the curves were drawn according to Eqs. (3.13) $(l)$ and (3.11)(s). For the sake of clarity the scale for $\mu_{s}^{0}$ was shifted by $5(k T)$ units. Eq. (3.11) describes the numerical data exactly, as expected, and Eq. (3.13) is accurate at the extremes but gives a slight overestimation around $(p v)^{0}=1$.

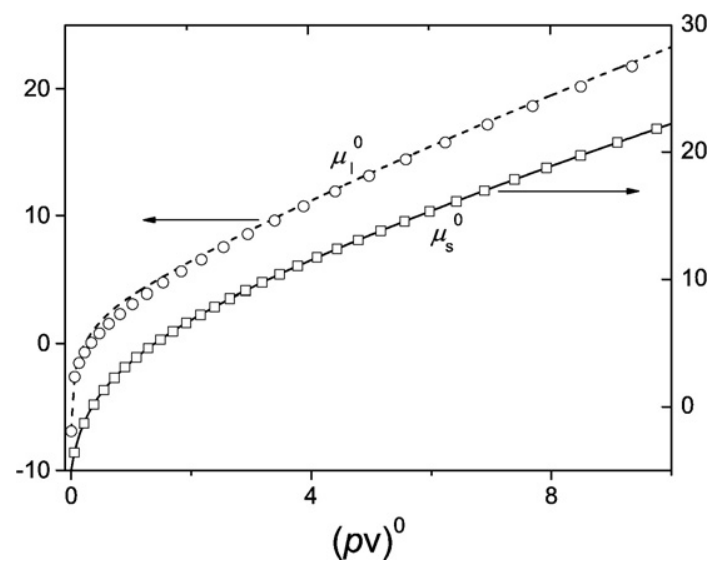

Fig. 3.4. The relation between $\mu^{0}$ and $(p v)^{0}$ for a hard-sphere liquid (index $l$ ) and a hardsphere solid (index $s$ ). Symbols are calculated from Eqs. (2.17) and (2.18). The curves are drawn according to Eqs. (3.13) $(l)$ and (3.11) (s).

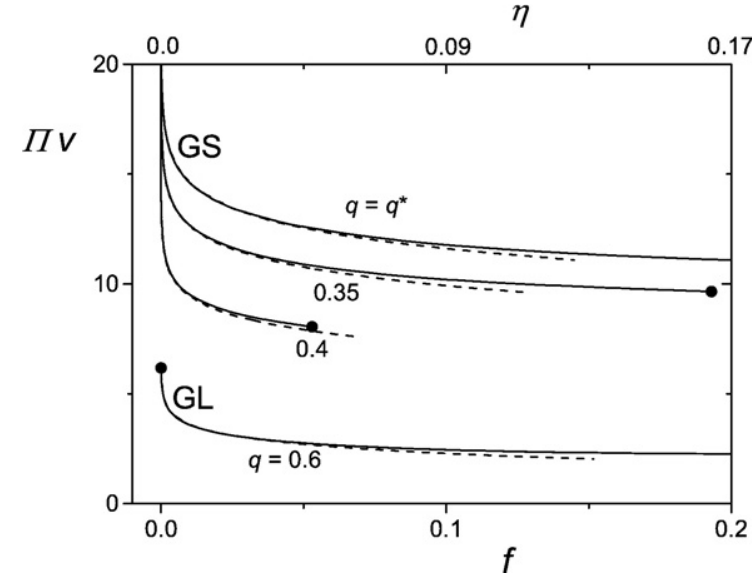

Fig. 3.5. The gas branches of three GS binodals from Fig. $3.1\left(q=q^{*}, 0.35,0.4\right)$ and of the GL binodal from Fig. 3.2 for $q=0.6$ (solid curves) as compared with the exclusion limit (dotted): Eq. (3.14) for GS, Eq. (3.15) for GL. The circles are triple-point coordinates, and are the same as in Figs. 3.1 and 3.2.

Substitution of Eqs. (3.8) and (3.11) into Eq. (3.10) gives an explicit equation for the gas branch of the GS equilibrium in the exclusion limit:

$\eta_{\mathrm{g}}=6.265(\Pi v)^{3} e^{\left(1.35-\zeta^{3}\right) \Pi v}$

Similarly, Eqs. (3.8), (3.10), and (3.13) give the analogous form for the GL gas branch:

$\ln \eta_{g}=3.65(\Pi v)^{0.8}-\Pi v \zeta^{3}+\Theta(1-\Pi v) \ln \Pi v$.

Fig. 3.5 shows the gas branches of three GS binodals from Fig. 3.1 ( $q=q^{*}, 0.35$, and 0.4 ; solid curves), and compares them with the limiting form (dotted) of Eq. (3.14). The agreement is excellent for $f<0.05$ or even beyond. This figure gives also the gas branch of the GL binodal for $q=0.6$ from Fig. 3.2; here the exclusion limit (Eq. (3.15), dotted) is accurate up to about $f=0.1$.

We note that the gas branches in Fig. 3.5 approach the limiting exclusion behavior faster than those for the condensed phases (crosses in Fig. 3.1, plusses in Fig. 3.2). For example, for $q=q^{*}$ the dotted curve in Fig. 3.5 coincides with the numerical binodal for $\Pi v$ above 12, whereas the solid branch in Fig. 3.1 does not reach the exclusion limit until $\Pi v$ above 20. A similar phenomenon is seen for the GL binodal for $q=0.6$, where the solid and dotted curves in Fig. 3.5 coincide down to $\Pi v \approx 2.5$, whereas the numerical L-branch in Fig. 3.2 deviates considerably from the plusses.

The reason is that Eq. (3.10) is a better approximation than Eq. (3.8), due to a compensation of errors. We illustrate this for the GS equilibrium (the reasoning for GL is analogous). When there is still a contribution due to the polymer in the solid phase, $\Pi v$ is higher than $(p v)_{s}^{0}$ because the difference $h_{g}-h_{s}$ in Eq. (3.1) is smaller than unity: Eq. (3.8) is not yet satisfied. The corresponding $\mu_{g}^{0} \approx \ln \eta$ follows from Eq. (3.1) as $\mu_{s}^{0}-\zeta^{3} \Pi v\left(g_{g}-g_{s}\right) \approx \mu_{s}^{0}-\zeta^{3}(p v)_{s}^{0}\left(g_{g}-g_{s}\right) / h_{g}-h_{s}$. Even when $g_{g}-g_{s}$ and $h_{g}-h_{s}$ are smaller than unity their ratio may be close to unity, so that Eq. (3.10) is rather accurate while Eq. (3.8) is not.

\subsubsection{Power-law behavior of triple and critical points}

The triple and critical points from Fig. 3.3 are replotted in Fig. 3.6, where all data are normalized on the cep. This figure shows how the six parameters $\tilde{f}_{g}^{t} \equiv f_{g}^{t} / f^{*}, \tilde{f}_{l}^{t} \equiv f_{l}^{t} / f^{*}, \tilde{f}_{s}^{t} \equiv f_{s}^{t} / f_{s}^{*}$ (triangles), $\tilde{f}^{c} \equiv f^{c} / f^{*}$ (diamonds), $\widetilde{\Pi v}^{t} \equiv(\Pi v)^{t} /(\Pi v)^{*}$ (circles), and $\widetilde{\Pi v}^{c} \equiv(\Pi v)^{c} /(\Pi v)^{*}$ (squares) depend on the inverse range $1 / \tilde{q} \equiv q^{*} / q$. The parameter $1 / \tilde{q}$ runs from unity in the cep to zero for high $q$. The same applies to $\widetilde{\Pi v}^{c}, \tilde{f}^{c}$, and $\tilde{f}_{g}^{t}$. The parameters $\widetilde{\Pi v}^{t}, \tilde{f}_{l}^{t}$, and $\tilde{f}_{s}^{t}$ go from unity (cep) to a nonzero final value. The symbols in Fig. 3.6 are the numerical results, the curves are analytical approximations as discussed below. 


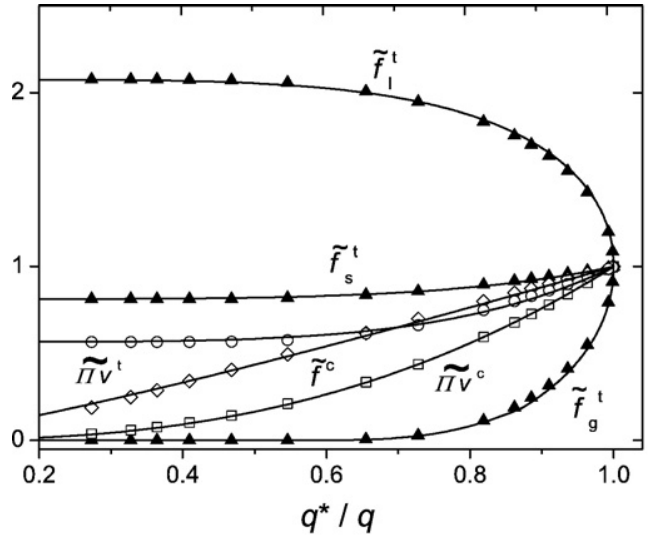

Fig. 3.6. Normalized colloid concentrations $\tilde{f}_{g}^{t}$, $\tilde{f}_{l}^{t}$ and $\tilde{f}_{s}^{t}$ (triangles) for the triple point and $\tilde{f}^{c}$ (diamonds) for the critical point, and the corresponding $\widetilde{\Pi v}^{t}$ (circles) and $\widetilde{\Pi v}^{c}$ (squares), as a function of the inverse (normalized) range $1 / \tilde{q} \equiv q^{*} / q$. Symbols are the numerical data for the same $q$ values as in Fig. 3.3 (and Table 5.1). Curves are analytical approximations as discussed in the text: Eq. (3.24) for $\tilde{f}_{g}^{t}$, Eq. (3.22) for $\tilde{f}_{l}^{t}$, Eq. (3.20) for $\tilde{f}_{s}^{t}$, Eq. (3.19) for $\Pi v^{t}$, and Eq. (3.18) for $\tilde{f}^{c}$ and $\widetilde{\Pi v}$.

The final high- $q$ levels for $\widetilde{\Pi v}^{t}, \tilde{f}_{l}^{t}$, and $\tilde{f}_{s}^{t}$ are easily derived from the exclusion limit. For thick depletion layers the polymer is nearly absent from the concentrated phases, and the thermodynamic properties are the same as without polymer: $(p v)_{l}^{0}=(p v)_{s}^{0}=$ $(p v)_{0}^{0}=6.081$ and $\mu_{l}^{0}=\mu_{s}^{0}=\mu_{0}^{0}=15.463$. This is also shown by the nearly vertical FS branches in Fig. $3.1(q=0.6)$ up to the triple point. Inserting $(p v)^{0}=6.081$ and $\mu^{0}=15.463$ into Eq. (3.10) (both versions are identical in this limit) gives $f_{g}^{t}$, so the high- $q$ limit $(q>0.6)$ of the triple point is:

$$
\begin{array}{ll}
(\Pi v)^{e}=6.081 & \ln f_{g}^{t}=15.463-6.081 \zeta^{3} \\
f_{l}^{e}=0.970 & f_{s}^{e}=1.185 .
\end{array}
$$

Hence the non-zero end values of the normalized parameters are $\widetilde{\Pi v}^{e}=6.081 / 10.729=0.567, \tilde{f}_{l}^{e} \equiv f_{f}^{0} / f^{*}=2.076$, and $\tilde{f}_{s}^{e} \equiv f_{s}^{0} / f_{s}^{*}=0.814$. Eq. (3.16b), which is the same as Eq. (3.14) with $(\Pi v)^{t}=6.081$, shows how $f_{g}^{t}$ decays to zero for $q>0.6$. For $0.6<q<0.4 \mathrm{Eq}$. (3.1) gives a more precise expression for $f_{g}^{t}$ (see also Eq. (3.24) below).

For the four middle curves in Fig. 3.6 the decay of the normalized parameters from unity in the cep to the end value can rather accurately be described as a power law:

$X-X^{e}=\left(1-X^{e}\right) \tilde{q}^{-x}$.

For those cases where $X^{e}$ is zero we get a very simple result:

$$
\left\{\begin{aligned}
\widetilde{\Pi v}^{c} & =\tilde{q}^{-2.6} \\
\tilde{f}^{c} & =\tilde{q}^{-1.2} \\
\widetilde{\Pi v}^{c} & =\left(\tilde{f}^{c}\right)^{2.2}
\end{aligned}\right.
$$

Fig. 3.6 shows that Eq. (3.18a,b) describe $(\Pi v)^{c}$ and $f^{c}$ as a function of $q$ quite well. In Fig. 3.3 we see that also Eq. (3.18c) is rather accurate. When $X^{e}$ is non-zero, we have to account for this end value:

$\widetilde{\Pi v}^{t}-\widetilde{\Pi v}^{e}=\left(1-\widetilde{\Pi v}^{e}\right) \tilde{q}^{-4.6} \quad \widetilde{\Pi v}^{t}=0.567+0.433 \tilde{q}^{-4.6}$

$\tilde{f}_{s}^{t}-\tilde{f}_{s}^{e}=\left(1-\tilde{f}_{s}^{e}\right) \tilde{q}^{-4.6} \quad \tilde{f}_{\mathrm{s}}^{t}=0.814+0.186 \tilde{q}^{-4.6}$.

The two middle curves in Fig. 3.6 were drawn according to Eqs. (3.19) and (3.20); they follow the numerical data remarkably well. We note that the exponents in Eqs. (3.19) and (3.20) are the same, so $\widetilde{\Pi v}^{t}$ as a function of $\tilde{f}_{s}^{t}$ gives a straight line from $(1,1)$ to $\left(\widetilde{\Pi v}^{e}, \tilde{f}_{s}^{e}\right)$, as also shown by the solid branch of the triple curve in Fig. 3.3. In Eqs. (3.18a) and (3.18b) the exponents are different, so $\widetilde{\Pi v}^{c}\left(\tilde{f}^{c}\right)$ in Eq. (3.18c) is not linear (see again Fig. 3.3).
The two remaining curves in Fig. (3.6), for $\tilde{f}_{g}^{t}$ and $\tilde{f}_{l}^{t}$, are not well described by a power law in $q$, because $f$ varies very steeply with $q$ around the cep. In other words, a plot $q(f)$ is nearly flat in this region. We use a different type of power law and consider the plot $\widetilde{\Pi v}^{t}(\tilde{f})$ in more detail. Such a plot was already given in Fig. 3.3. It is seen that $\widetilde{\Pi v}^{t}$ $(\tilde{f})$ around the cep is nearly parabolic. We use a modification of Eq. (3.17) by considering a power law in the parameter $(\tilde{f}-1) /\left(\tilde{f}^{e}-1\right)$, which runs from unity at $f=f^{e}$ to zero at the cep. When we apply this modified version to the liquid branch, we obtain

$\frac{1-\widetilde{\Pi v}^{t}}{1-\widetilde{\Pi v}^{e}}=\left(\frac{\tilde{f}_{l}-1}{\tilde{f}_{l}^{e}-1}\right)^{2.1} \quad \widetilde{\Pi v}^{t}=1-0.372\left(\tilde{f}_{l}-1\right)^{2.1}$.

When the exponent is taken as 2 , we have a pure parabola, which would describe the liquid branch reasonably. The exponent 2.1 works slightly better, as shown by the liquid branch of the triple curve in Fig. 3.3. When in Eq. (3.21) $\widetilde{\Pi v}^{t}$ is eliminated using Eq. (3.19), we obtain the explicit dependence $f_{l}^{t}(q)$ :

$\left(\frac{\tilde{f}_{l}-1}{\tilde{f}_{l}^{e}-1}\right)^{2.1}=1-\tilde{q}^{-4.6} \quad \tilde{f}_{l}^{t}=1+1.076\left(1-\tilde{q}^{-4.6}\right)^{0.48}$.

The upper curve in Fig. (3.6), drawn according to Eq. (3.22), gives a very good description of the numerical data $f_{l}^{t}(q)$.

For the gas branch in Fig. 3.3 a simple parabola $1-\widetilde{\Pi v}^{t} \sim\left(1-\tilde{f}_{g}\right)^{2}$ works quite well around the cep, but its width is slightly different from that of the liquid branch. For small $f_{\mathrm{g}}$ a parabolic description breaks down because then the exponential exclusion limit of Eq. (3.14) is appropriate. We combine these two limits as follows:

$\tilde{f}_{g}^{t}= \begin{cases}13.41(\Pi v)^{3} e^{\left(1.35-\zeta^{3}\right) \Pi v} & 6.08<\Pi v<8.53 \\ 1-1.81 \sqrt{1-\Pi v /(\Pi v)^{*}} & 8.53<\Pi v<(\Pi v)^{*}\end{cases}$

In Eq. (3.23a) we substitute $\zeta=1+q$ from Eq. (3.19) with $(\Pi v)^{e}=6.08$, $\zeta=1+q^{*}[(\Pi v-6.08) / 4.65]^{-1 / 4.6}=1+0.46(\Pi v-6.08)^{-0.22}$ in order to find $f_{g}^{t}(\Pi v)$. Fig. 3.3 shows that Eq. (3.23) describes the gas branch excellently. We can eliminate $\Pi v$ from Eq. (3.23), using Eq. (3.19), to find $f_{g}^{t}(q)$ :

$\tilde{f}_{g}^{t}= \begin{cases}\left.13.41(\Pi v)^{3} e^{\left(1.35-\zeta^{3}\right.}\right) \Pi v & \tilde{q}>1.15 \\ 1-1.19 \sqrt{1-\tilde{q}^{-4.6}} & 1<\tilde{q}<1.15\end{cases}$

where in Eq. (3.24a) we use $\Pi v=6.08+4.65 \tilde{q}^{-4.6}$ (Eq. (3.19)) and $\zeta=1+$ $q^{*} \tilde{q}$. Again we see (Fig. 3.6) good agreement between the analytical description and the numerical data.

Finally, we show in Fig. 3.7 the $f_{g}^{t}(q)$ data in another way: $\log f_{g}^{t}$ against $\zeta^{3}$. We choose this representation because the high- $q$ limit

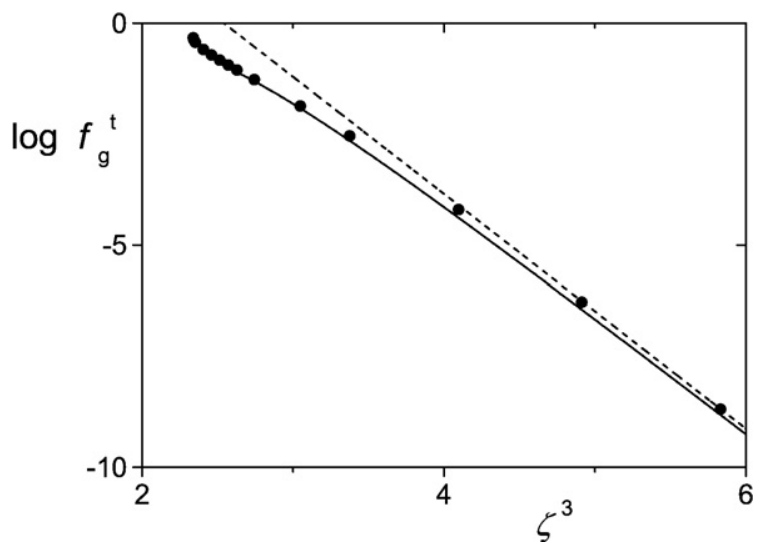

Fig. 3.7. The gas branch of the triple curve in the representation $\log f_{\mathrm{g}}^{t}$ as a function of $\zeta^{3}$. Symbols are the numerical data, the solid curve is Eq. (3.24), and the dashed line is the limiting form Eq. (3.16b). 
is then a straight line, according to Eq. (3.16b). This line is indicated in Fig. 3.7 and works well for $q>0.6, \zeta^{3}>4$. The more accurate version of Eq. (3.14) (or Eq. (3.24a)) for the exclusion limit applies for $q>0.4, \quad \zeta^{3}>2.7$. For $q^{*}<q<0.4$ we need also the 'parabolic' version of Eq. (3.24b).

\subsubsection{GL binodals and spinodals}

Fig. 3.8 gives numerical binodals (filled circles) for five values of $q$, with analytical approximations (solid curves) as discussed below. Also the exclusion limit ( $q=\infty$, open diamonds) for the binodal is shown. The filled circles for $q=0.35,0.4$, and 0.6 correspond to the numerical data in Fig. 3.2; in addition we give the numerical data for $q=0.45$ and $q=1$. Fig. 3.8 gives also numerical triple points (open circles) and critical points (open squares), plus the analytical results for the triple curve (Eqs. (3.22) and (3.23)) and the critical curve (Eq. (3.18c)), indicated as the dashed curves. The dotted curves in Fig. 3.8 are spinodals, see below.

The L-branch of each binodal is rather accurately described by a parabola with its minimum in the critical point and its end point on the liquid branch of the triple curve:

$\frac{\Pi v}{(\Pi v)^{c}}-1=\left(\frac{(\Pi v)^{t}}{(\Pi v)^{c}}-1\right)\left(\frac{f-f^{c}}{f^{t}-f^{c}}\right)^{2}$.

For the liquid branch we substitute $f^{t}=f_{l}^{t}$. When the numerical values for $(\Pi v)^{c}$ and $(\Pi v)^{t}$ are used in Eq. (3.25), the curves follow the numerical binodal very precisely. In Fig. 3.8 we see some slight deviations because we aim at a fully analytical solution, so we inserted our analytical results for the triple and critical point.

For low $q$, below roughly $q=0.4$, the gas branch can also be described by a parabola, but with a different width because the triple curve is not fully symmetric around $f^{*}$. The curves for the gas branches for $q=0.35$ and 0.4 were obtained from Eq. (3.25), substituting the (analytical) $f_{g}^{t}$ for $f^{t}$. They work satisfactorily; the deviations in the G-branch are, however, stronger for $q=0.4$ than for $q=0.35$.

For higher $\mathrm{q}$ the gas branch becomes very asymmetric, and a parabolic description breaks down. For the dilute part we have an alternative using the exclusion limit, as derived in Eq. (3.15). The analytical G-binodals in Fig. 3.8 for $q=0.45$ and up were obtained by

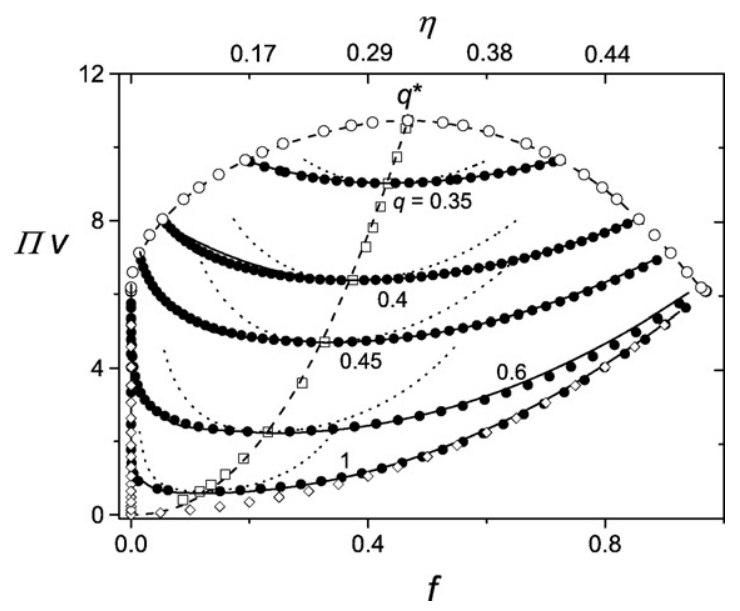

Fig. 3.8. The fluid part of Fig. 3.3 with triple points (open circles), critical points (open squares), and GL binodals (solid curves) and spinodals (dotted) for five $q$ values. Symbols (filled circles for the binodals) are numerical data, the curves are analytical. The equations for the triple and critical curves (dashed) are given in Section 3.2.2, the binodals (solid) follow Eq. (3.25) $(q=0.35,0.4)$ or Eq. (3.26) (higher $q$ ), and the spinodals (dotted) are given by Eq. (3.28). The open diamonds for $q=\infty$ correspond to the exclusion limit of Eq. (3.8), in combination with Eq. (2.18a) combining the exclusion limit (Eq. (3.15)) with the inverted form of the (extrapolated) parabolic L-binodal as follows:

$$
\left\{\begin{array}{l}
f_{g}=\max \left(f_{g}^{\text {exc }}, f_{g}^{\text {par }}\right) \\
f_{g}^{\text {exc }}=\exp \left\{3.657(\Pi v)^{0.799}-\Pi v \zeta^{3}+\Theta(1-\Pi v) \ln \Pi v\right\} \\
f_{g}^{\text {par }}=f^{c}-\left(f_{l}^{t}-f^{c}\right) \sqrt{\left[\Pi v-(\Pi v)^{c}\right] /\left[(\Pi v)^{t}-(\Pi v)^{c}\right]}
\end{array}\right.
$$

whereby the analytical approximations for $(\Pi v)^{t}(q)$ (Eq. (3.19)), $f_{l}^{t}(q)$ (Eq. (3.22)), $(\Pi v)^{c}(q)$ and $f^{c}(q)$ (Eq. (3.18)) were used. Eq. (3.26) describes the numerical binodals quite well, although there is of course a discontinuity where the two limiting forms of Eq. (3.26) meet.

When we define $\Delta f=f-f^{c}, \mathrm{D} f=f^{t}-f^{c}, \Delta \Pi=\Pi-\Pi^{c}$ and $\mathrm{D} \Pi=\Pi^{t}-\Pi^{c}$, where both $\Delta f / D f$ and $\Delta \Pi / D \Pi$ run from zero at the critical point to unity at the (liquid side of the) triple point, we have from Eq. (3.25) $\Delta f \sim(\Delta \Pi)^{0.5}$. Close to the critical point and in dilute polymer solutions we may translate this as $\Delta \eta \sim(\Delta \varphi)^{0.5}$ : the critical exponent [55] for 'fixed q' free-volume theory is 0.5 . Recent simulations [56] suggest a flatter binodal, with a critical exponent 0.33 . In chapter 6 we will find that 'variable $q$ ' free-volume theory also gives a smaller critical exponent and a flatter binodal.

We conclude this section with some remarks about spinodal curves. Spinodals are calculated from the condition $d p / d f=0$ (or $d \mu$ ) $d f=0)$. With $p v=(p v)^{0}+\Pi v h($ Eq. $(2.31))$ we find

$$
(\Pi v)^{s p}=\frac{(p v)_{1}^{0}}{f \beta_{1}} .
$$

Explicit expressions for the derivatives $\beta_{1}$ and $(p v)_{1}^{0} \equiv d(p v)_{f}^{0} / d f$ are given in Eqs. (2.9) and (2.19).

It is possible to derive analytical approximations for the spinodals. We mention one result:

$$
\frac{(\Pi v)^{s p}}{(\Pi v)^{c}}=1+\frac{1}{2}\left(\frac{f}{f^{c}}-1\right)^{2}
$$

Because of the prefactor, the spinodal is narrower than the binodal. We plotted the spinodal curves according to Eq. (3.28) for five values of $q$ in Fig. 3.8 as the dotted curves.

\section{Radius of gyration and overlap concentration}

The $\Pi v(f)$ diagrams as a function of $q=\delta / a$ discussed in the previous chapter 3 may be converted to $\varphi(f)$ or $\varphi(\eta)$ diagrams as a function of $q_{R}=R / a$. This will be done in chapter 5 for 'fixed $q$ ' (where $\delta$ is independent of the polymer concentration $\varphi$, and $\Pi$ proportional to $\varphi$ ) and in chapter 6 for 'variable $q$ ' (where $\delta$ decreases with increasing $\varphi$, and $\Pi$ increases more strongly with $\varphi$ ). For this conversion we have to relate $q$ and $\Pi v$ to $q_{R}$ and $\varphi$. We will see that for 'fixed $q$ ' $q$ is somewhat smaller than $q_{R}$ (roughly $q=0.9 q_{R}^{0.9}$, see Eq. (5.5), and $\Pi v$ is simply related to $y=\varphi / \varphi_{\text {ov }}$ and $q_{R}: \Pi v=y / q_{R}^{3}$ (Eq. (5.8)). For 'variable $q$ ' these relations have to be extended (Section 6.2): $q$ then decreases with increasing $y$, and $\Pi v$ is no longer linear in $y$. Nevertheless, also in this case the same parameters play a role and $y(f)$ diagrams as a function of $q_{R}$ can be obtained.

In order to convert such normalized $y\left(f, q_{R}\right)$ diagrams to $\varphi(f, R)$ or $\varphi(\eta, N)$ diagrams for a given chain length $N$ and solvency $\boldsymbol{v}$, we need to know how $R$ and $\varphi_{\text {ov }}$ depend on $N$ and $\boldsymbol{v}$; this relation is discussed in the present chapter 4 .

\subsection{Radius of gyration}

The well-known scaling relations for the radius of gyration $R$ as a function of chain length are $R \sim N^{1 / 2}$ in mean field (mf) and $R \sim N^{3 / 5}$ for excluded-volume chains (ev). We need not only the correct exponents but also the numerical prefactors. In addition, it would be useful to 
have an equation for intermediate solvencies. We start from the linear expansion coefficient $\alpha_{e}$ introduced by Flory [57]

$R=\alpha_{e} n / \sqrt{6} \quad n \equiv \sqrt{N}$

We express $R$ in units of the Kuhn length. For convenience, we use the parameter $n$ rather than $N$ itself. Flory [57] derived an equation for $\alpha_{e}$. In terms of $\boldsymbol{v}=1-2 \chi$, where $\chi$ is the Flory-Huggins parameter (which is 0.5 in a theta solvent and smaller in a better solvent), this relation may be written as

$\alpha_{e}^{5}-\alpha_{e}^{3}=\frac{3}{2} \boldsymbol{v} n$.

This equation is implicit in $\alpha_{\mathrm{e}}$. Clearly $\alpha_{\mathrm{e}}=1$ for $\boldsymbol{v}=0$ and $\alpha_{\mathrm{e}} \sim(\boldsymbol{v} n)^{1 / 5}$ for large $\boldsymbol{v} n$, so the correct Flory-exponent $\boldsymbol{v}$ in $R \sim N^{v}=n^{2 v}$ is recovered in both $\mathrm{mf}$ and ev.

We obtain an explicit approximation for $\alpha_{\mathrm{e}}$ when we replace $\alpha_{e}^{3}$ by $\alpha_{e}^{5 / 2}$, solving the resulting quadratic equation in $\alpha_{e}^{5 / 2}$ to find $2 \alpha_{e}^{5 / 2}=1+\sqrt{1+6 \boldsymbol{v} n}$. This solution underestimates $\alpha_{e}$ from Eq. (4.2) slightly. A somewhat higher numerical prefactor of $\boldsymbol{v} n$ gives a very accurate approximation:

$2 \alpha_{e}^{5 / 2}=1+\sqrt{1+6.5 v n}$.

Fig. 4.1 shows $\alpha_{e}$ as a function of $n$ according to this approximation (solid curves) for $\boldsymbol{v}=0,0.03,0.1,0.2,0.6$, and 1 , corresponding to $\chi=0.5,0.485,0.45,0.4,0.2$, and 0 . The symbols in Fig. 4.1 give the numerical solution of Eq. (4.2). The approximation of Eq. (4.3) is very good indeed, overestimating $\alpha_{\mathrm{e}}$ by $0.4 \%$ for $\boldsymbol{v} n=100$, and underestimating it by the same amount for $\boldsymbol{v} n=10$. Even very close to theta conditions $(\chi=0.485)$ Eq. (4.3) works quite well. The dotted curve in Fig. 4.1 gives, for $\boldsymbol{v}=1$, the limiting behavior $\alpha_{e}=1.10(\boldsymbol{v} n)^{1 / 5}$, obtained by omitting the unity terms in Eq. (4.3).

In order to find the relation $R(n)$ we combine Eqs. (4.1) and (4.3):

$R=0.309 n[1+\sqrt{1+6.5 v n}]^{2 / 5}$.

For $\boldsymbol{v}=0$ this reduces to $R=0.408 n=\sqrt{N / 6}$. Eq. (4.4) is a rather simple relation, which applies to the whole solvency range, from theta conditions $(\boldsymbol{v}=0)$ to athermal solvents $(\boldsymbol{v}=1)$.

Fig. 4.2 (solid curves) shows $R(n)$ according to Eq. (4.4) and compares it with the numerical solution of Eqs. (4.1) and (4.2) (symbols), for the same set of $\boldsymbol{v}$ 's as in Fig. 4.1. The quality of the approximation is the same as in Fig. 4.1.

The radius of gyration $R$ follows from $n$ through an easy explicit relation. Conversely, for given $R$ the value of $n$ is obtained by solving Eq. (4.4) implicitly. For our purpose it is convenient to have an explicit

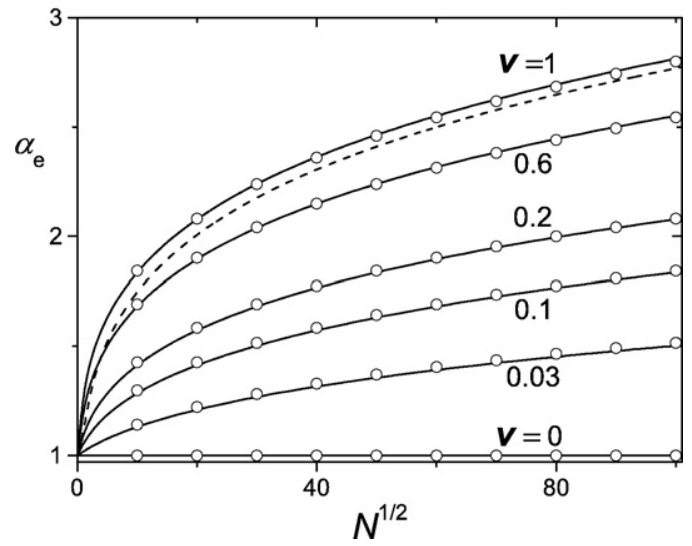

Fig. 4.1. The linear expansion coefficient $\alpha_{e}$ as a function of the square root of the chain length for, from bottom to top, $\boldsymbol{v}=0,0.03,0.1,0.2,0.6$, and 1 . Symbols are the numerical solution of Eq. (4.2), solid curves are the approximation of Eq. (4.3). The dotted curve is the high- $\boldsymbol{v} n$ limit $\alpha_{e}=1.10(v n)^{1 / 5}$ for $\boldsymbol{v}=1$.

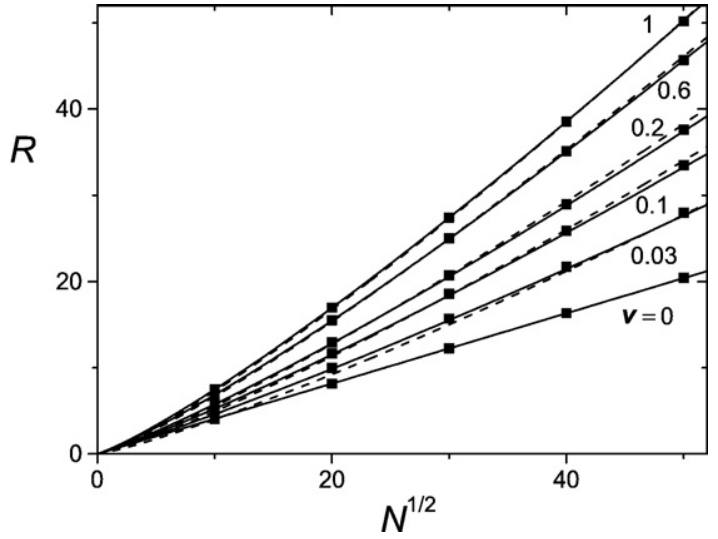

Fig. 4.2. The radius of gyration $R$ as a function of the square root of the chain length for, from bottom to top, $\boldsymbol{v}=0,0.03,0.1,0.2,0.6$, and 1 . Symbols are the numerical solution of Eqs. (4.1) and (4.2), solid curves follow Eq. (4.4). The dashed curves are the scaling relations of Eq. (4.5).

approximation $n(R)$ to Eq. (4.4). For $\boldsymbol{v}=1$ the high- $n$ limit of Eq. (4.4) is $R=0.449 n^{1.2}$. Comparison with the numerical result Eqs. (4.1), (4.2) shows a slight underestimation, which we correct by adjusting the numerical constant to 0.46 . The next step is the $\boldsymbol{v}$-dependence, which for very high $\boldsymbol{v}$ should be $R \sim \boldsymbol{v}^{0.2}$. For finite chain lengths a slightly smaller exponent describes the numerical solvency dependence better. We use the following scaling relation to describe the trends:

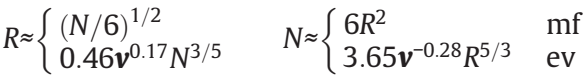

The dashed curves in Fig. 4.2 show that Eq. (4.5) is rather precise over most of the range for $\boldsymbol{v}$. For example, for $N=400$ it is accurate within $2 \%$ for $\boldsymbol{v}=1$ and about $3 \%$ for $\boldsymbol{v}=0.1$. Only for very small $\boldsymbol{v}(0.03$ in Fig. 4.2$)$ do we see the expected deviations, but even then Eq. (4.5) is not too bad.

\subsection{Overlap concentration}

The overlap concentration is defined as $\varphi_{\text {ov }}=N / V_{\text {coil }}$ with $V_{\text {coil }}=4 \pi R^{3} / 3$ :

$\varphi_{\mathrm{ov}}=\frac{3}{4 \pi} \frac{N}{R^{3}}$

Inserting Eq. (4.4) into Eq. (4.6) we find $\varphi_{\text {ov }}(n, v)$ :

$\varphi_{\mathrm{ov}}=\frac{8.09}{n}[1+\sqrt{1+6.5 \boldsymbol{v} n}]^{-6 / 5}$.

As Eq. (4.4), this simple relation is valid over the whole range of solvencies, including a theta solvent.

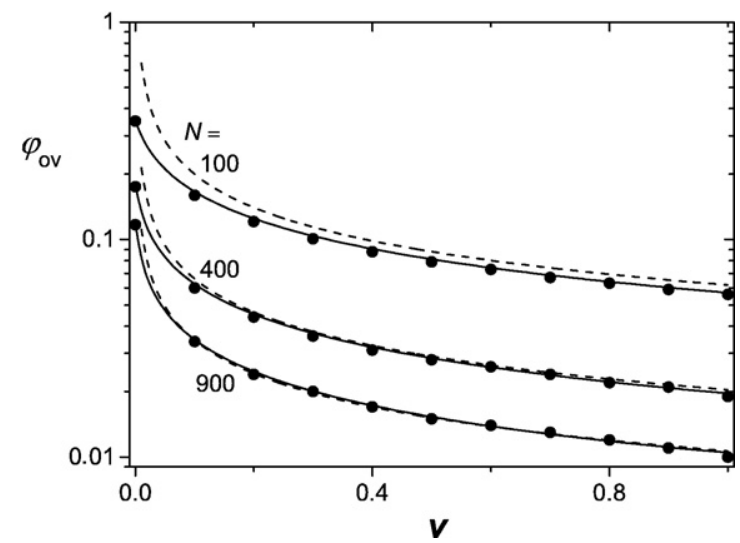

Fig. 4.3. The overlap concentration as a function of the solvency parameter $\boldsymbol{v}$, for $N=100$, 400,900 . Symbols are numerical data from Eqs. (4.1), (4.2), and (4.6). Solid curves follow Eq. (4.7), dashed curves are the scaling relation Eq. (4.8b). 


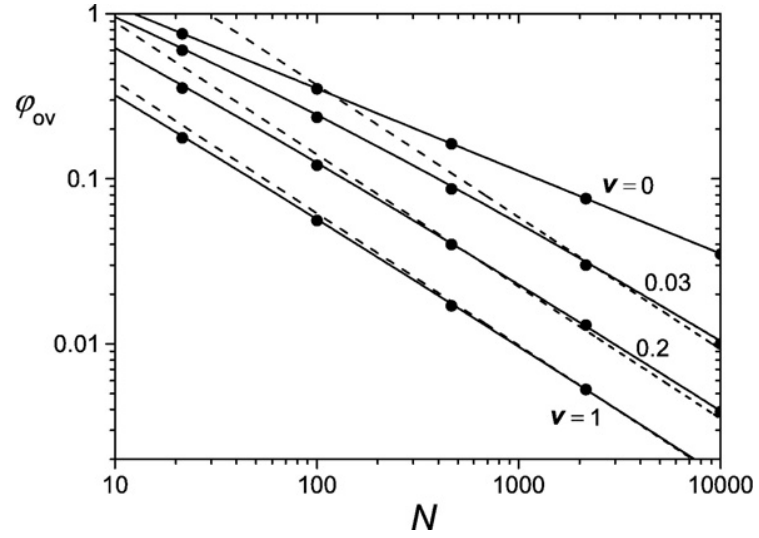

Fig. 4.4. The overlap concentration as a function of the chain length, for four values $\boldsymbol{v}$ as indicated. Symbols are numerical data from Eqs. (4.1), (4.2), and (4.6). The solid curves are Eq. (4.7), the dashed lines $(\boldsymbol{v}>0)$ are the scaling relation Eq. (4.8b).

With the scaling relation Eq. (4.5) we may approximate $\varphi_{\mathrm{ov}}$ as:

$\varphi_{\mathrm{ov}} \approx\left\{\begin{array}{ll}1.43 R^{-1} \approx 3.51 N^{-1 / 2} & \mathrm{mf} \\ 0.87 \boldsymbol{v}^{-0.28} R^{-4 / 3} \approx 2.46 \boldsymbol{v}^{-0.51} N^{-4 / 5} & \mathrm{ev}\end{array}\right.$.

Fig. 4.3 shows $\varphi_{\text {ov }}$ as a function of $\boldsymbol{v}$ for three chain lengths: $n=10$, 20, 30 or $N=100,400,900$. Symbols are the exact results from Eq. (4.6) in combination with Eqs. (4.1) and (4.2). The solid curves are calculated from Eq. (4.7), the dashed curves are the scaling relation Eq. (4.8b). Eq. (4.7) describes the numerical data quite well, over the whole range of solvencies. For long chains the scaling relation is adequate over most of the solvency range; only for very small $\boldsymbol{v}$, where Eq. (4.8b) diverges, are some deviations visible. For smaller $N$ these deviations become more pronounced. For $N=900 \mathrm{Eq}$. (4.8b) is accurate above $\boldsymbol{v} \approx 0.03$ or $\chi$ below $\chi \approx 0.485$, for $N=400$ the validity range is $\boldsymbol{v}>0.1, \chi<0.45$, and for short chains $(N=100)$ Eq. (4.8) is reasonable for $\boldsymbol{v}>0.2, \chi<0.4$.

Fig. 4.4 gives a double-logarithmic plot $\varphi_{\text {ov }}(N)$ for four solvencies. Symbols are again obtained from Eqs. (4.1), (4.2) and (4.6); the solid curves follow Eq. (4.7). In a theta solvent $\varphi_{\text {ov }}$ is inversely proportional to the square root of the chain length. In a good solvent the scaling for long chains is $\varphi_{\mathrm{ov}} \sim N^{-4 / 5}$ (dashed lines according to Eq. (4.8b)), and this scaling is adequate over nearly the whole range $N>10$ for $\boldsymbol{v}=1$ and over the range $N>100$ for $\boldsymbol{v}=0.2$. For $\boldsymbol{v}=0.03$ the scaling is valid only for long chains $(N>1000)$.

We have now identified how $\varphi_{\text {ov }}$ depends on $N$ and $R$ for any solvency. These ingredients will be used in chapters 5 and 6 to study the dependence of colloid/polymer phase diagrams on chain length and solvency.

\section{Phase diagrams for 'fixed $q$ ' in terms of the polymer concentration}

In chapter 3 we discussed phase diagrams in terms of the parameters $\Pi v$, which is the osmotic work to insert a sphere (without depletion layer) into a polymer solution, and the size ratio $q=\delta / a$, where $\delta$ is the depletion thickness around a sphere of radius $a$. No relation between $\Pi v, q$ and the radius of gyration $R$ of the polymer coils and the polymer volume fraction $\varphi$ was needed in chapter 3 . In this chapter we establish these relations for the case that the polymer length scale $\delta$ is constant (i.e., 'fixed $q$ '). Such relations are relevant for practical purposes since experimentally polymer concentrations $\varphi$ are measured, rather than the reduced concentration $y=\varphi / \varphi_{\mathrm{ov}}$. The present 'fixed $q$ ' treatment is expected to be a reasonable approximation for relatively dilute polymer solutions (colloid limit).

We consider first the reduced parameters $q_{R}=R / a$ and $y=\varphi / \varphi_{\mathrm{ov}}$, where $\varphi_{\mathrm{ov}}$ is the overlap concentration; in this chapter we restrict ourselves to dilute polymer solutions $(y<1)$. We show that there is a direct relation between $q$ and $q_{R}$ and that $\Pi v$ may be simply expressed in $q_{R}$ and $y$. Hence, the $\Pi v(q)$ diagrams of chapter 3 can be converted to $y\left(q_{R}\right)$ diagrams, which are universal in the sense that they do not depend on $R, a$, and $\varphi_{\mathrm{ov}}$ separately; only ratios are involved. In order to find phase diagrams in terms of real concentrations $\varphi=y \varphi_{\mathrm{ov}}$ and the particle radius $a=R / q_{R}$, we have to specify $R$ and $\varphi_{\mathrm{ov}}$; clearly, these parameters depend on the chain length and solvency. This dependence was discussed in the previous chapter 4 .

\subsection{Relation between $q=\delta / a$ and $q_{R}=R / a$}

We first focus on the depletion thickness next to a flat plate. In the dilute limit the depletion thickness does not depend on the polymer concentration; we use the symbol $\delta_{0}$ for this dilute limit. The relation between $\delta_{0}$ and $R$ is well known $[58,59]$ :

$\delta_{0}=p R \quad p=\left\{\begin{array}{ll}2 / \sqrt{\pi}=1.128 & \mathrm{mf} \\ 2 x / \sqrt{\pi}=1.071 & \mathrm{ev}\end{array}\right.$.

The constant $x$ equals $3 / 4+(3 / 8) \ln 2+\pi / 8-\pi / \sqrt{48}=0.9492$ $[30,59]$. The depletion thickness $\delta_{0}$ at a plate in the dilute limit is thus slightly higher than $R$, by about $13 \%$ in a theta solvent and $7 \%$ in a good solvent.

The depletion thickness $\delta$ around a sphere is smaller than $\delta_{0}$ next to a plate, due to curvature effects. It is convenient to define not only the size ratio $q$, as before, but also the size ratios $q_{0}$ and $q_{R}$ :

$q=\frac{\delta}{a} \quad q_{R}=\frac{R}{a} \quad q_{0}=\frac{\delta_{0}}{a}=p q_{R}$.

The relation between $q$ and $q_{R}$ has been derived by Aarts et al. [30] and Louis et al. [38] for mf-chains (i.e., theta conditions) and by Hanke et al. [59] for ev-chains in a good solvent. We rewrite these relations in terms of $q\left(q_{0}\right)$ :

$\zeta^{3}=(1+q)^{3}=\left\{\begin{array}{ll}1+3 q_{0}+(3 \pi / 4) q_{0}^{2} & \mathrm{mf} \\ 1+3 q_{0}+3 c_{2} q_{0}^{2}+3 c_{3} q_{0}^{3}+\ldots & \mathrm{ev}\end{array}\right.$.

where the numerical constants in Eq. (5.3b) are $c_{2}=[1-5 \pi / 8+17 / 36+$ $(\pi / 4) \sqrt{3}] / 1.071^{2}=0.7577$ and $c_{3}=-(3 \sqrt{\pi})[1673 \pi / 48-551 / 15-40 \pi /$ $\sqrt{3}] / 1.071^{3}=-0.0325$.

Fig. 5.1 gives a plot $q_{0}(q)$. The dashed curve is Eq. (5.3a) ( $\left.\mathrm{mf}\right)$, the solid curve is Eq. (5.3b) (ev). As expected $q_{0}$ is larger than $q$, but the effect is not very strong: over the $q$-range shown the ratio $q_{0} / q$ is between 1 and 4/3. Moreover, the difference between $\mathrm{mf}$ and ev is small, especially for $q$ smaller than 1 , which is the most relevant region.

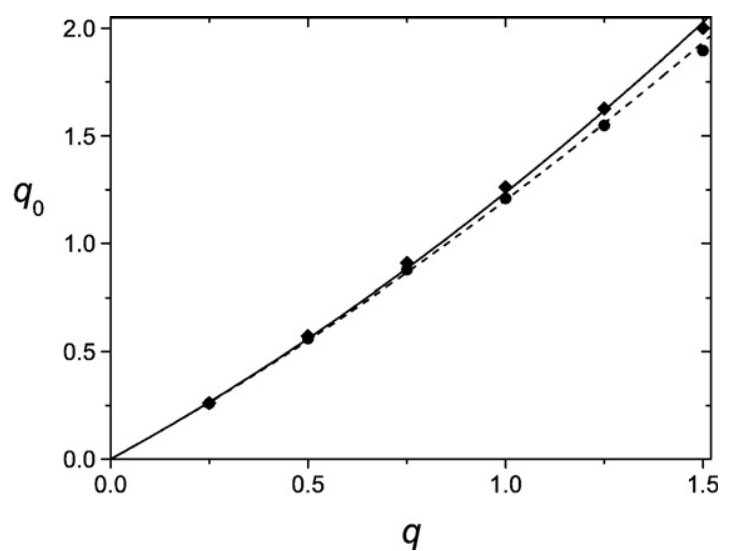

Fig. 5.1. The dependence $q_{0}(q)$ for a theta solvent (dashed curve) and a good solvent (solid) according to Eq. (5.3). The symbols are the approximate power-law behavior of Eq. (5.5). 
We have seen in chapter 3 that the critical endpoint (cep) is the central feature of the phase diagram. The values of $q^{*}, q_{0}^{*}$, and $q_{R}^{*}$ in the cep are given by

$q^{*}=0.3275 \quad q_{0}^{*}=\left\{\begin{array}{l}0.3502 \\ 0.3534\end{array} \quad q_{R}^{*}= \begin{cases}0.3104 & \mathrm{mf} \\ 0.3299 & \mathrm{ev}\end{cases}\right.$

As in chapter 3, it is useful to normalize the parameters on those at the cep: $\tilde{q}=q / q^{*}, \tilde{q}_{0}=q_{0} / q_{0}^{*}, \tilde{q}_{R}=q_{R} / q_{R}^{*}$. It turns out that the relation between $q$ and $q_{0}$ or $q_{R}$ in Eq. (5.3) may be approximated as a simple power law:

$\tilde{q}_{0}=\tilde{q}_{R}=\left\{\begin{array}{l}\tilde{q}^{1.11} \\ \tilde{q}^{1.14}\end{array} \quad q=\left\{\begin{array}{cc}0.842 q_{0}^{0.9}=0.938 q_{R}^{0.9} & \mathrm{mf} \\ 0.815 q_{0}^{0.88}=0.865 q_{R}^{0.88} & \mathrm{ev}\end{array}\right.\right.$

The circles (mf) and diamonds (ev) in Fig. 5.1 give this power law. The agreement with the exact data of Eq. (5.3) is excellent. Therefore we use the simple power-law form of Eq. (5.5) for the conversion between the $q$ parameters.

Table 5.1 gives numerical data for $q, q_{0}$, and $q_{R}$; these will be used in the present chapter 5 and in the next chapter 6 . The first column shows the $q$ values used in chapter 3; the first two values are below the cep, the third (bold-face) corresponds to the cep, and the further values in this column are those for which (in chapter 3 and in the present chapter) triple and critical points are indicated in the various figures. Columns 2 and 3 give $q_{0}$ and $q_{R}=q_{0} / p$ for a theta solvent, columns 4 and 5 those for a good solvent.

\subsection{Relation between $\Pi v$ and reduced polymer concentration $y$}

We define the reduced (external) polymer concentration as

$y=\varphi / \varphi_{\mathrm{ov}}$

where $\varphi_{\text {ov }}$ is given by Eq. (4.6), (4.7), or (4.8).

In the dilute polymer regime $(y<1)$, which corresponds to the colloid limit, we assume that the osmotic pressure $\Pi$ is given by the ideal law:

$\Pi=\varphi / N$

With $\varphi=\mathrm{y} \varphi_{\mathrm{ov}}$ we find from Eqs. (5.7) and (4.6) $\Pi=(3 / 4 \pi) y / R^{3}$. Multiplication by $v=(4 \pi / 3) a^{3}$ gives

$\Pi v=y / q_{R}^{3}$

which shows that $\Pi v$ may be written in terms of reduced parameters only.

Table 5.1

Numerical data for $q, q_{0}$, and $q_{R}$ as used in most of the figures

\begin{tabular}{lllll}
\hline$q$ & $q_{0}(\mathrm{mf})$ & $q_{R}(\mathrm{mf})$ & $q_{0}(\mathrm{ev})$ & $q_{R}(\mathrm{ev})$ \\
\hline 0.2000 & 0.2025 & 0.1795 & 0.2014 & 0.1880 \\
0.2600 & 0.2710 & 0.2402 & 0.2716 & 0.2536 \\
$\mathbf{0 . 3 2 7 5}$ & $\mathbf{0 . 3 5 0 2}$ & $\mathbf{0 . 3 1 0 4}$ & $\mathbf{0 . 3 5 3 4}$ & $\mathbf{0 . 3 2 9 9}$ \\
0.3280 & 0.3508 & 0.3108 & 0.3539 & 0.3305 \\
0.3300 & 0.3531 & 0.3130 & 0.3564 & 0.3328 \\
0.3400 & 0.3650 & 0.3235 & 0.3687 & 0.3443 \\
0.3500 & 0.3770 & 0.3341 & 0.3811 & 0.3558 \\
0.3600 & 0.3889 & 0.3447 & 0.3935 & 0.3675 \\
0.3700 & 0.4009 & 0.3553 & 0.4060 & 0.3791 \\
0.3800 & 0.4130 & 0.3660 & 0.4186 & 0.3908 \\
0.4000 & 0.4372 & 0.3874 & 0.4438 & 0.4144 \\
0.4500 & 0.4983 & 0.4416 & 0.5075 & 0.4739 \\
0.5000 & 0.5601 & 0.4963 & 0.5723 & 0.5344 \\
0.6000 & 0.6857 & 0.6077 & 0.7045 & 0.6578 \\
0.7000 & 0.8137 & 0.7211 & 0.8399 & 0.7842 \\
0.8000 & 0.9437 & 0.8363 & 0.9780 & 0.9132 \\
0.9000 & 1.0755 & 0.9531 & 1.1185 & 1.0444 \\
1.0000 & 1.2089 & 1.0713 & 1.2613 & 1.1777 \\
1.2000 & 1.4800 & 1.3117 & 1.5527 & 1.4497 \\
\hline
\end{tabular}

Bold-face numbers correspond to the cep.

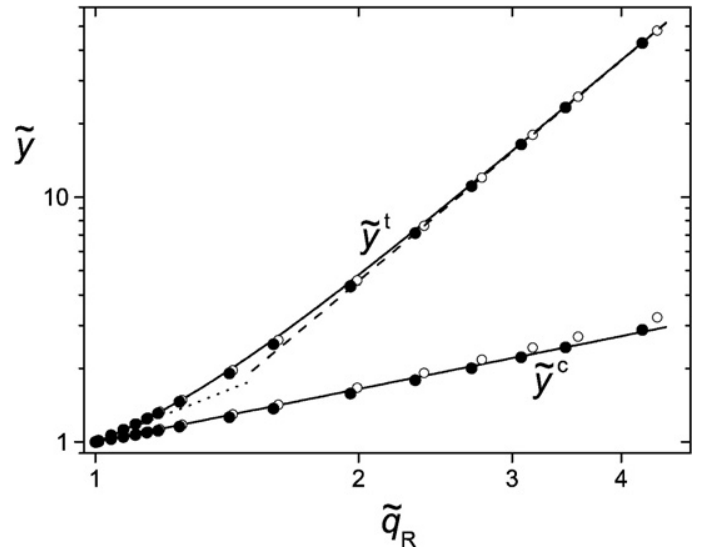

Fig. 5.2. Normalized critical points $\tilde{y}^{c}$ and triple points $\tilde{y}^{t}$ as a function of $\tilde{q}_{R}$, in a double-logarithmic representation. Closed symbols are numerical data for a theta solvent, open symbols are for a good solvent; they correspond to the 17 highest $q$ values in Table 5.1. The solid curves are analytical: Eq. (5.10) for cp and Eq. (5.11) for tp. The dashed line is the high-q limit $\tilde{y}^{t}=0.567 \tilde{q}_{R}{ }^{3}$; the dotted line is the other limit $\tilde{y}^{t}=\tilde{q}_{R}{ }^{1.4}$ close to the cep.

In the cep $(\Pi v)^{*}=10.729$ and $q_{R}^{*}$ is given by Eq. (5.4c). Hence, $y=y^{*}$ in the cep is

$y^{*}=(\Pi v)^{*}\left(q_{R}^{*}\right)^{3}=\left\{\begin{array}{cc}0.3208 & \mathrm{mf} \\ 0.3854 & \mathrm{ev}\end{array}\right.$.

Because $y^{*}$ is well below unity, the cep is located within the dilute polymer concentration regime and the present 'fixed $q$ ' description is adequate, at least around the cep (colloid limit). In chapter 6 we will see that a more general 'variable $q$ ' treatment where non-ideal effects are taken into account gives roughly the same $y^{*}$ as in Eq. (5.9); around the cep non-ideal effects do not yet play an important role. Through Eq. (5.8) we can convert $\Pi v$ at given $q$ to $y$. To that end, we need the conversion between $q$ and $q_{R}$, for which we use Eq. (5.5); numerical data are given in Table 5.1.

We first apply Eq. (5.8) to the critical and triple points. We do this in the normalized form by dividing $y^{c}(\mathrm{cp})$ and $y^{t}(\mathrm{tp})$ by $y^{*}: \tilde{y}^{c}=y^{c} / y^{*}$ and $\tilde{y}^{t}=y^{t} / y^{*}$. Similarly, we use $\tilde{q}_{R}=q_{R} / q_{R}^{*}$ as a normalized size ratio.

Fig. 5.2 shows $\tilde{y}^{c}$ and $\tilde{y}^{t}$ as a function of $\tilde{q}_{R}$ on a double-logarithmic scale. Filled symbols are for mf, open symbols for ev; they correspond to the $q$ values ( $q^{*}$ and up) as given in the first column of Table 5.1. Although the $\tilde{q}_{R}$ values for $\mathrm{mf}$ and ev at given $q$ are slightly different (see again Table 5.1), $\tilde{y}^{c}$ and $\tilde{y}^{t}$ for both conditions essentially coincide on a single curve. Using the analytical approximations derived in chapter 3 in combination with $\tilde{y}=\widetilde{\Pi v} \tilde{q}_{R}^{3}$, we can easily find the equations for the critical and triple curves.

For the critical curve we have $\widetilde{\Pi v}=\tilde{q}^{-2.6}$, see Eq. (3.18). With Eq. (5.5b) (ev) this may be written as $\widetilde{\Pi v}=\tilde{q}_{R}^{-2.28}$. Hence,

$\tilde{y}^{c}=\tilde{q}_{R}^{0.72}$

On a double-logarithmic scale, this gives the straight critical line drawn in Fig. 5.2. It fits the numerical data nicely for both $\mathrm{mf}$ and ev, although for high $q$ some slight deviations are visible, especially in a good solvent.

Along the triple curve $\widetilde{\Pi v}^{t}$ depends only weakly on $q$ according to Eq. (3.19): it drops from unity in the cep to 0.567 for $q$ above 0.6. Hence, for high $q$ we have $\tilde{y}^{t}=0.567 \tilde{q}_{R}^{3}$, which is the limiting straight line (dashed) in Fig. 5.2. For smaller $q$ we can apply Eq. (3.19), which would give $\widetilde{\Pi v}^{t}=0.567+0.433 \tilde{\mathrm{q}}_{R}^{-4.03}$ from Eq. (5.5b). In Fig. 5.2 a slightly smaller exponent in the correction term works better. The solid triple curve in this figure was drawn according to

$\tilde{y}^{t}=\tilde{q}_{R}^{3}\left(0.567+0.433 \tilde{q}_{R}^{-3.7}\right)$. 
This equation describes the numerical $y^{t}\left(q_{R}\right)$ data excellently over the whole range of $q_{R}$, both for $\mathrm{mf}$ and $\mathrm{ev}$.

A very important feature of Fig. 5.2 is that, whereas $(\Pi v)^{c}$ decreases strongly with increasing $q_{R},(\Pi v)^{c} \sim q_{R}^{-2.28}$, the parameter $y^{c}$ increases $\left(y^{c} \sim q_{R}^{0.72}\right)$. For the triple point $(\Pi v)^{t}$ goes weakly down with increasing $q_{R}$ (and becomes independent of $q_{R}$ for high $q$ ), whereas $y^{t}$ increases strongly $\left(y^{t} \sim q_{R}^{3}\right.$ for high $\left.q\right)$. The reason is clear: the factor $q_{R}^{3}$ in $y=\Pi v q_{R}^{3}$ (Eq. (5.8)) is responsible for the (relatively weak) increase of $y^{c}$ and the strong increase of $y^{t}$ with $q_{R}$.

We note that Eq. (5.11) has the form $z=x^{\kappa}\left[A+(1-A) x^{-\mu}\right]$, which describes the crossover from a dependence $z=x^{\lambda}$ for $z$ and $x$ close to unity to $z=A x^{\kappa}$ for high $x$. From evaluating $d \ln z / d \ln x=d z / d x$ at $x=1$ it is easily shown that $\lambda=\kappa-\mu(1-A)$. For the parameters in Eq. (5.11) this gives $\lambda=1.40$, hence $\tilde{y}^{t}=\tilde{q}_{R}^{1.4}$ close to the cep; this limiting form is also shown in Fig. 5.2 as the dotted line. In chapter 6 we shall use a similar crossover from $\tilde{q}_{R}^{\lambda}$ to $A \tilde{q}_{R}^{\kappa}$ for $\tilde{y}^{t}$ and $\tilde{y}^{c}$ in the case of 'variable $q$ '.

\subsection{Phase diagrams in terms of the reduced polymer concentration $y$}

\subsubsection{FS binodals}

Fig. 5.3 shows FS binodals in the representation $y(f)$ for $q=0.2,0.26$, $0.3275\left(=q^{*}\right), 0.35,0.4$, and 0.5 in a good solvent. The corresponding $q_{R}$ values are $0.188,0.254,0.3299\left(=q_{R}^{*}\right), 0.356,0.414$, and 0.534 (see Table 5.1). We note that a $y(f)$ diagram for a theta solvent gives nearly the same figure, with minor quantitative differences because $q_{R}$ in a theta solvent is, at given $q$, slightly smaller than in a good solvent (see again Table 5.1). Fig. 5.3 may be compared with Fig. 3.1, displaying $\Pi v(f)$ binodals for the same set of $q$ 's (except for the highest $q$, which is 0.6 in Fig. 3.1).

The most striking difference between the two figures is that Fig. 5.3 is more or less a vertical mirror image of Fig. 3.1: the curves for the lowest $q$ lie on top in Fig. 3.1 and at the bottom in Fig. 5.3, and with increasing $q$ the curves go down in Fig. 3.1 and up in Fig. 5.3. Another aspect is that the curves for high $q$ converge to the same limit in Fig. 3.1, whereas the curves in Fig. 5.3 diverge. In both cases the factor $q_{R}^{3}$ in Eq. (5.8) is responsible for this different behavior.

As in Fig. 3.1, the circles in Fig. 5.3 are the triple-point values where the FS binodals for $q>q^{*}$ show a discontinuous jump in the $F$ branch and a kink in the $S$ branch. For $q=q^{*}$ (cep) the gas branch of the FS binodal has again an inflection point at the fluid side of the cep. In Fig. 3.1 the cep is the triple point with the highest $(\Pi v)^{t}$, whereas in Fig. 5.3 the cep corresponds to the lowest $y^{t}$, and for high $q$ the triple point in terms of $y^{t}$ diverges, as discussed above. Both effects are due to the factor $q_{R}^{3}$ in Eq. (5.8). We note that for $q_{R}=0.5$ and up $y^{t}$ is above unity, outside the dilute limit. Then the 'fixed $q$ ' model breaks down and has to be replaced by 'variable $q$ ' (chapter 6).

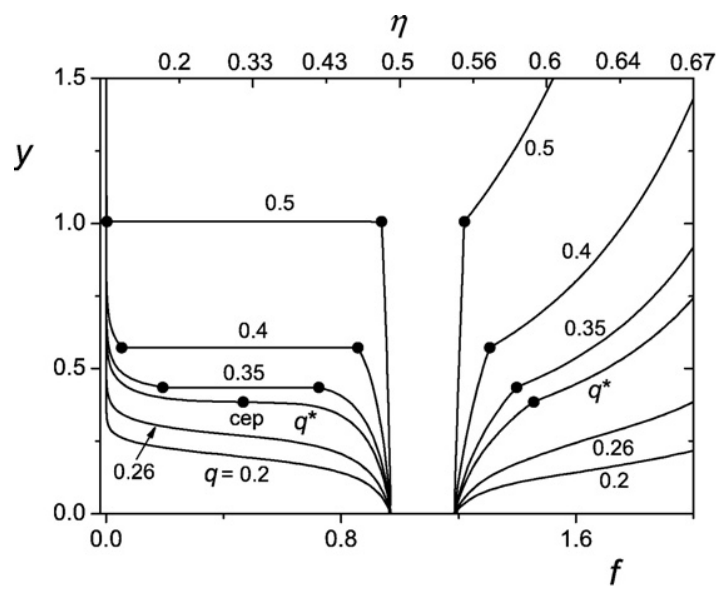

Fig. 5.3. FS binodals $y(f)$ for a good solvent and for $q=0.2,0.26,0.3275\left(q^{*}\right), 0.35,0.4$, and 0.5 , corresponding to $q_{R}=0.188,0.254,0.330,0.356,0.414$, and 0.534 , respectively. The circles indicate the triple points for the four highest $q$ values; the triple point for $q=q^{*}$ is the cep.

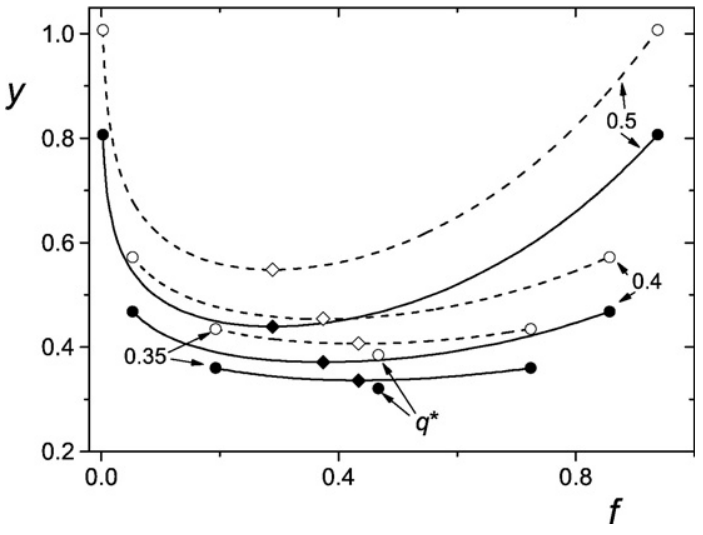

Fig. 5.4. GL binodals $y(f)$ for $q=0.3275$ ( $q^{*}$, point binodal), $0.35,0.4$, and 0.5 , for both a theta solvent (solid curves) and a good solvent (dashed). The circles are triple points (closed for mf, open for ev) and the cep; the open circles for a good solvent are the same as in Fig. 5.3. The diamonds (closed for mf, open for ev) are the critical points.

\subsubsection{GL binodals}

Fig. 5.4 presents GL binodals $y(f)$ for $q=q^{*}$ (point binodal) and $q=0.35,0.4$, and 0.5 . Open circles are the triple points of Fig. 5.3 (only the fluid parts), diamonds are the critical points. In this case we give data for both a good solvent (dashed curves, open symbols) and for a theta solvent (solid curves, closed symbols). Fig. 5.4 may be compared with Fig. 3.2, which shows binodals in the $\Pi v(f)$ representation.

Each individual GL binodal (at fixed $q$ ) in Fig. 5.4 has the same shape as in Fig. 3.2 but with increasing $q$ the order is inverted, as for the FS binodals (Fig. 5.3) and the triple points. The data in Fig. 5.4 clearly demonstrate that, starting from the cep, the critical points $y^{c}(f)$ increase with $q$, whereas in Fig. $3.2(\Pi v)^{c}(f)$ goes down with increasing $q$; this effect was already discussed in the derivation of Eq. (5.10). For better solvency the binodal curves shift to higher $y$. This is due to chain expansion, which also increases the depletion thickness. Whereas in Fig. (3.2) we could indicate the high- $q$ limit for binodals $\Pi v(f)$, this is impossible in Fig. (5.4) because there is no such limit: due to the factor $q_{R}^{3}$ in Eq. (5.8) the $y(f)$ binodals diverge for high $q$. However, that is the regime where the 'fixed $q$ ' treatment is expected to fail.

\subsubsection{Critical and triple points}

Fig. 5.5 gives critical and triple points $y(f)$, and is the $y$-variant of Fig. 3.3, for the same set of q's. Unlike in Fig. 3.3 (and in Figs. 5.3 and 5.4) we use normalized quantities $\tilde{y}^{c}$ and $\tilde{y}^{t}$, because in Fig. 5.2 we

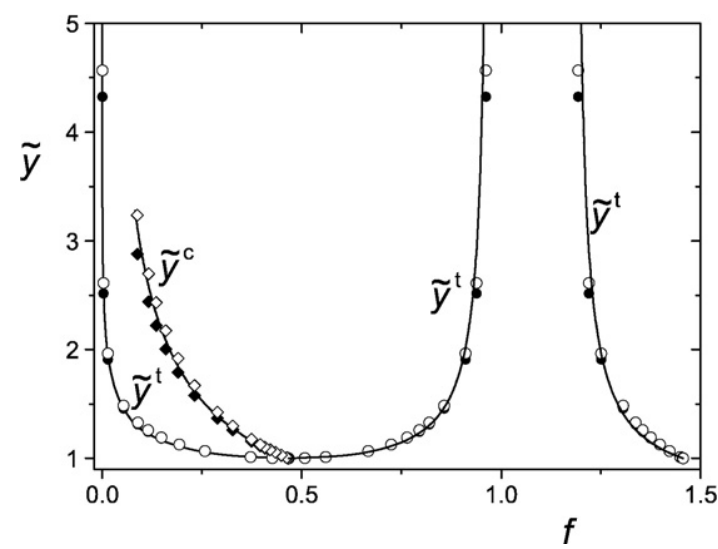

Fig. 5.5. Normalized triple points $\tilde{y}^{t}(f)$ (circles) and critical points $\tilde{y}^{c}(f)$ (diamonds) for the standard set of $q$ 's (Table 5.1). Closed symbols are for mf, open ones for ev. The highest triple point indicated is for $q=0.6$. The curves are analytical: Eq. (5.10) for $\tilde{y}^{c}\left(q_{R}\right)$, Eq. (5.11) for $\tilde{y}^{t}\left(q_{R}\right)$, Eq. (3.18) for $f^{c}(q)$, Eq. (3.20) for $f_{s}^{t}(q)$, Eq. (3.22) for $f_{l}^{t}(q)$, and Eq. (3.24) for $f_{g}^{t}(q)$. 


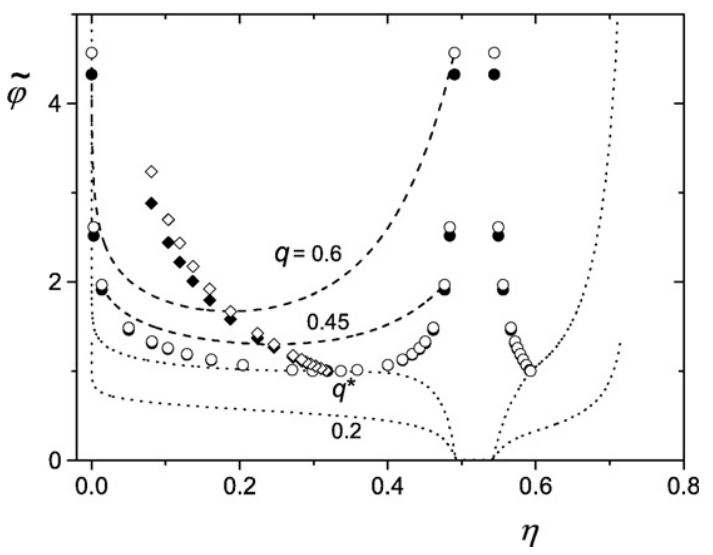

Fig. 5.6. Normalized phase diagram $\tilde{\varphi}(\eta)$ for a constant chain length $N$. Triple points (circles) and critical points (diamonds) are for the standard set of $q$ 's (Table 5.1). Closed symbols are for mf, open ones for ev. The two GL binodals (dashed curves) are for $q=0.45$ and $q=0.6$, the two FS binodals (dotted) are for $q=0.2$ and $q=q^{*}$. The four binodals are for a good solvent, but those for a theta solvent would look essentially the same.

saw that then the data for mf and ev nearly collapse onto a single curve. Closed symbols in Fig. 5.5 are for a theta solvent ( $\mathrm{mf}$ ), open ones for a good solvent (ev). We see that $\tilde{y}^{t}$ diverges strongly $\left(\sim q_{R}{ }^{3}\right)$ for high $q$ : the highest triple point indicated in the figure is for $q=0.6$. Also $\tilde{y}^{c}$ diverges but more slowly $\left(\sim q_{R}^{0.72}\right)$ : the highest critical point indicated is for $q=1.2$.

The curves shown in Fig. 5.5 are analytical on the basis of Eqs. (5.10) and (5.11) for $\tilde{y}^{c}\left(q_{R}\right)$ and $\tilde{y}^{t}\left(q_{R}\right)$, and Eqs. (3.18), (3.20), (3.22), (3.24) for $f^{c}(q), f_{s}^{t}(q), f_{l}^{t}(q)$, and $f_{g}^{t}(q)$, respectively. Although in principle we could eliminate $q$ and/or $q_{R}$ and find explicit expressions $y(f)$, as we did for $\Pi v(f)$ in Section 3.2.2, we will not give these expressions. The curves in Fig. 5.5 were obtained parametrically by expressing both the $y$ 's and the $f$ 's in $q_{R}$, using Eq. (5.5) to convert $q$ in $f(q)$ to $q_{R}$. It is clear that the analytical approximations describe the critical and triple points in Fig. 5.5 quite well.

\subsection{Phase diagrams in terms of the external polymer concentration $\varphi$}

In the previous section we discussed $y(f)$ phase diagrams as a function of $\mathrm{q}$ and/or $q_{R}$, where $y=\varphi / \varphi_{\mathrm{ov}}$. Experimentally, one measures the polymer concentration $\varphi$ and not the relative concentration $y$, even though it is common practice in this field that experimentalists plot phase diagrams in $(y, \eta)$ coordinates. This becomes especially relevant when one changes the solvency, for instance by changing solvent composition or temperature.

In order to find $\varphi=y \varphi_{\mathrm{ov}}$, we have to specify the overlap concentration, which depends on the polymer chain length and the solvency (see chapter 4). We split this topic in two parts: first we find $\varphi^{*}=y^{*} \varphi_{\mathrm{ov}}^{*}$ for the cep, and then we consider the parameter $\tilde{\varphi}=\varphi / \varphi^{*}$ with respect to the cep. Clearly,

$\tilde{\varphi}=\tilde{y} \tilde{\varphi}_{\mathrm{ov}}$

where $\tilde{\varphi}_{\mathrm{ov}}=\varphi_{\mathrm{ov}} / \varphi_{\mathrm{ov}}^{*}$.

We also want to know how the phase diagrams change with the value of the particle radius $a$ (when $R$ or $N$ and $\boldsymbol{v}$ are given), or with $N$ and $\boldsymbol{v}$ (when $a$ is fixed). We treat these issues separately in Sections 5.4.1 and 5.4.2.

\subsubsection{Phase diagrams at constant chain length $N$}

We choose constant chain length $N$ (rather than constant radius of gyration) because then we have accurate explicit expressions to calculate $R$ (Eq. (4.4)) and $\varphi_{\mathrm{ov}}$ (Eq. (4.7)). When constant $\mathrm{R}$ is chosen instead, we would have to solve the implicit Eqs. (4.4) and (4.7) to find $N$ and $\varphi_{\text {ov }}$.

For given $n=\sqrt{N}$ and $\boldsymbol{v}$ we can immediately find $\varphi^{*}=y^{*} \varphi_{\mathrm{ov}}^{*}$ and $a^{*}=R / q_{R}^{*}$ in the cep. From Eqs. (4.4) and (4.7) we obtain

$\varphi^{*}=\frac{8.09 y^{*}}{n}[1+\sqrt{1+6.5 v n}]^{-6 / 5} \approx \begin{cases}1.13 N^{-1 / 2} & \mathrm{mf} \\ 0.95 v^{-0.51} N^{-4 / 5} & \mathrm{ev}\end{cases}$

$a^{*}=\frac{0.309 n}{q_{R}^{*}}[1+\sqrt{1+6.5 \boldsymbol{v} n}]^{2 / 5} \approx\left\{\begin{array}{ll}4.25 N^{1 / 2} & \mathrm{mf} \\ 4.21 \boldsymbol{v}^{0.17} N^{3 / 5} & \mathrm{ev}\end{array}\right.$.

In the scaling approximations of Eq. (5.13) we used Eqs. (4.5) and (4.8) and we inserted the numerical values of $y^{*}$ (Eq. (5.9)) and $q_{R}^{*}$ (Eq. (5.4)).

Next we consider how a and $\varphi_{\text {ov }}$ vary outside the cep. For the present case (constant $N$ ) the situation is extremely simple: $\varphi_{\mathrm{ov}}$ is constant (at given $\boldsymbol{v}$ ) and $a$ is inversely proportional to $q_{R}$ :

$$
\tilde{\varphi}_{\mathrm{ov}}=1 \quad \tilde{\varphi}=\tilde{y} \quad \tilde{a}=\tilde{q}_{R}^{-1} .
$$

Eq. (5.14b) implies that our previous results of Fig. 5.5 can immediately be transferred to the present case of constant N. Fig. 5.6 gives the normalized phase diagram $\tilde{\varphi}(\eta)$. In such a normalized plot the cep is, by definition, situated at $\tilde{\varphi}=1$. The critical and triple points are the same as in Fig. 5.5 (except for the conversion from $f$ to $\eta$ ). We added two GL binodals for $q=0.45$ and 0.6 (similar to Fig. 5.4, but in a normalized form) and two FS binodals for $q=0.2$ and $q=q^{*}$ (as in Fig. 5.3).

Normalized diagrams $\tilde{\varphi}(\eta)$ as in Fig. 5.6 may be converted to $\varphi(\eta)$ at given $\mathrm{N}$ and $\boldsymbol{v}$ by multiplying $\tilde{\varphi}$ by $\varphi^{*}$ as given by Eq. (5.13a). In Fig. 5.7 we plot non-normalized phase diagrams $\varphi(\eta)$ for five solvencies and $N=400$. In order not to overcrowd the figure, we display only the critical points (diamonds) and triple points (circles). The cep values for $\varphi^{*}$ (Eq. (5.13a)) are indicated in the legend. The approximate scaling result is $\varphi^{*}=0.95 \boldsymbol{v}^{-0.51} N^{-0.8}=0.0079 \boldsymbol{v}^{-0.51}$ for $\boldsymbol{v}>0$. Outside the cep we have $\varphi=\varphi^{*} \tilde{y}$, with $\tilde{y}^{c}$ and $\tilde{y}^{t}$ given by Eqs. (5.10) and (5.11), respectively.

Eqs. (5.13b) and (5.14c) also provide the information about the particle radii for which a stable colloidal liquid phase exists in the example of Fig. 5.7; the cep values $a^{*}$ are given in the legend. In a good solvent a liquid exists for larger particle sizes than in a theta solvent: $a^{*}=26.3$ for $\boldsymbol{v}=0$ and $a^{*}=51.4$ for $\boldsymbol{v}=1$. The particle radius at given $N$ ( $=400$ in this example) should be smaller than $a^{*}$ for a liquid to be stable. The value of $a$ corresponding to the symbols at given $q$ in

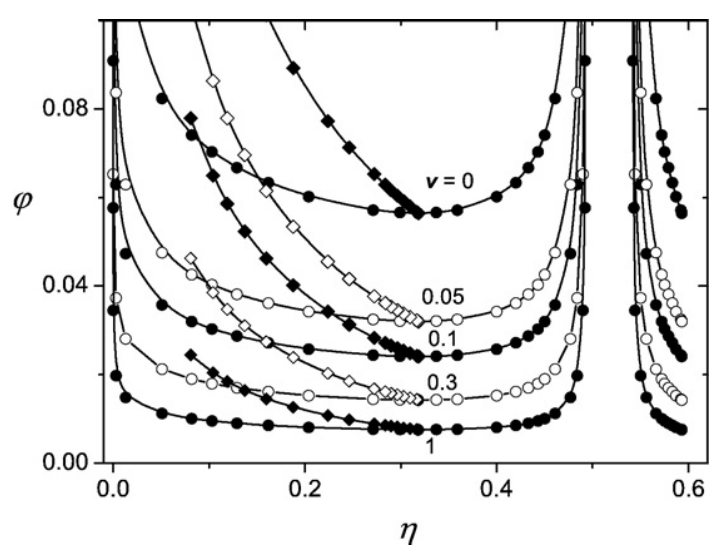

Fig. 5.7. Triple points (circles) and critical points (diamonds) for $N=400$ and five solvencies: from top to bottom $\boldsymbol{v}=0,0.05,0.1,0.3,1$, which with Eq. (4.4) corresponds to $R=8.2,10.5,11.5,13.7$, and 16.9, respectively. The set of $q$ 's is the standard set (Table 5.1). The $\varphi^{*}$ values (Eq. (5.13a)) in the cep are 0.056, 0.032, 0.024, 0.014, and 0.0076, respectively. The $a^{*}$ values (Eq. (5.13b)) are $26.3,31.7,34.9,41.5$, and 51.4. 
Fig. 5.7 follow from $a=a^{*} \tilde{q}_{R}^{-1}$, where $q_{R}$ is given in Table 5.1: as $\tilde{q}_{R}$ increases along the triple and critical curves, $a$ becomes smaller than $a^{*}$. For example, for the highest $q(=1.2)$ in Table $5.1 \tilde{q}_{R}=4.23(\boldsymbol{v}=0)$ or $\tilde{q}_{R}=4.39(\boldsymbol{v}>0)$, so for the highest critical point in Fig. $5.7 a \approx 0.23 a^{*}$.

\subsubsection{Phase diagrams at constant particle radius a}

When $a$ is fixed, $q_{R}$ can only be varied by adjusting the polymer radius $R$ through the chain length $N$ or the solvency parameter $\boldsymbol{v}$. This implies that $\varphi_{\text {ov }}$ changes as $q_{R}$ increases. In the cep $\left(R^{*}=a q_{R}^{*}\right)$ we find $R^{*}$ directly, but for obtaining $N^{*}$ we have to solve the implicit Eq. (4.4). Also for computing $\varphi_{\mathrm{ov}}^{*}$ from Eq. (4.7) (which is explicit in $N$ and $\boldsymbol{v}$ but not in $R$ and $\boldsymbol{v}$ ) we have an implicit equation. In a numerical scheme this poses no problem, but it makes insight in the trends more difficult.

We simplify things by using in this case only the scaling relations of Eqs. (4.5) and (4.8), whereby $R$ can be explicitly converted to $N$ and $\varphi_{\mathrm{ov}}^{*}$ (and conversely). In this high- $R$ (high- $N$ ) limit we obtain $\varphi^{*}$ in the cep from $\varphi^{*}=\mathrm{y}^{*} \varphi_{\mathrm{ov}}^{*}$ where $\varphi_{\mathrm{ov}}^{*}$ is found from Eq. (4.8) by substituting $R^{*}=a q_{R}^{*}$ :

$\varphi^{*}=\left\{\begin{array}{l}1.48 a^{-1} \\ 1.47 v^{-0.28} a^{-4 / 3}\end{array} \quad R^{*}=\left\{\begin{array}{ll}0.31 a \\ 0.33 a\end{array} \quad N^{*}= \begin{cases}0.58 a^{2} & \mathrm{mf} \\ 0.58 v^{-0.28} a^{5 / 3} & \mathrm{ev}\end{cases}\right.\right.$

Outside the cep we have $\varphi_{\mathrm{ov}} \sim R^{-1}$ (mf) or $\varphi_{\mathrm{ov}} \sim R^{-4 / 3}(\mathrm{ev})$ :

$\tilde{\varphi}_{\mathrm{ov}}=\left\{\begin{array}{l}\tilde{R}^{-1}=\tilde{q}_{R}^{-1} \\ \tilde{R}^{-4 / 3}=\tilde{q}_{R}^{-4 / 3}\end{array} \quad \tilde{\varphi}=\left\{\begin{array}{l}\tilde{y} \tilde{q}_{R}^{-1} \\ \tilde{y} \tilde{q}_{R}^{-4 / 3}\end{array} \quad \tilde{R}=\tilde{q}_{R} \quad \tilde{N}= \begin{cases}\tilde{q}_{R}^{2} & \mathrm{mf} \\ \tilde{q}_{R}^{5 / 3} & \mathrm{ev}\end{cases}\right.\right.$

Fig. 5.8 gives the normalized plot $\tilde{\varphi}(\eta)$ for a constant particle radius $(a=30)$; it is the analogue of Fig. 5.6 for constant $N$. The most conspicuous difference is that, starting from the cep, $\tilde{\varphi}^{c}$ in Fig. 5.6 goes up with increasing $q$, whereas in Fig. 5.8 it goes down. This is because of the factor $\tilde{q}_{R}^{-1}(\mathrm{mf})$ and $\tilde{q}_{R}^{-4 / 3}(\mathrm{ev})$ in Eq. (5.16b), which overcompensates the increase of $\tilde{y}^{c}\left(=\tilde{q}_{R}{ }^{0.72}\right.$ according to Eq. (5.10)). Hence, in a theta solvent (closed diamonds in Fig. 5.8) $\tilde{\varphi}^{c}=\tilde{q}_{R}^{-0.23}$ goes weakly down, whereas the decrease in a good solvent (open diamonds) is stronger $\left(\tilde{q}_{R}^{-0.61}\right)$.

The same factor $\tilde{q}_{R}^{-1}(\mathrm{mf})$ and $\tilde{q}_{R}^{-4 / 3}(\mathrm{ev})$ applies to the triple points, but here this additional factor is not enough to reverse the trend $\left(y^{t} \sim q_{R}^{3}\right)$, and $\tilde{\varphi}^{t}$ in Fig. 5.8 still increases rather strongly with $q_{R}$, though less than in Fig. 5.6. We have shown only the symbols for critical and triple points in Fig. 5.8, without connecting curves, but it is clear that also here a fully analytical description is feasible by combining Eq. (5.16b) with Eq. (5.10) (cp) or Eq. (5.11) (tp).

Fig. 5.8 shows one GL binodal ( $q=0.6$, dashed) and two FS binodals ( $q=0.2$ and $q=q^{*}$, dotted). These three binodals are for a good solvent,

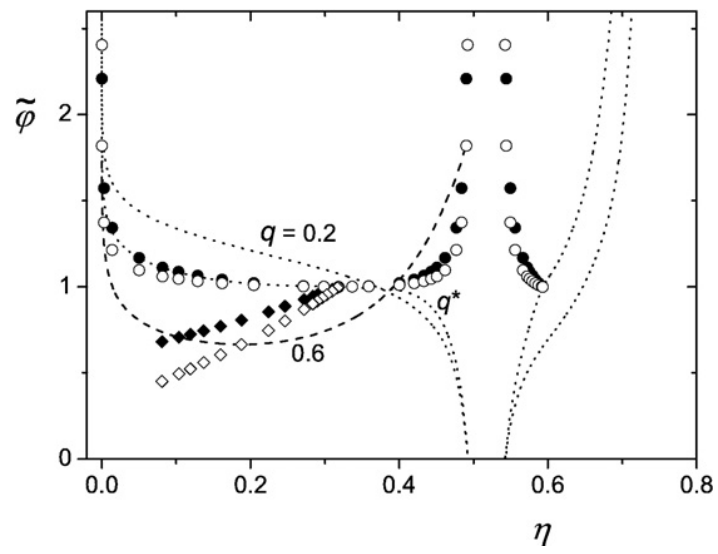

Fig. 5.8. Normalized phase diagram $\tilde{\varphi}(\eta)$ for a constant particle radius $a=30$. Triple points (circles) and critical points (diamonds) are for the standard set of $q$ 's. Closed symbols are for $\mathrm{mf}$, open ones for $\mathrm{ev}$. The highest triple point indicated is $q=0.6$ for $\mathrm{mf}$ and $q=0.7$ for ev. The GL binodal (dashed curve) is for $q=0.6$, the two FS binodals (dotted) are for $q=0.2$ and $q=q^{*}$. The three binodals are for a good solvent, but those for a theta solvent would look essentially the same.

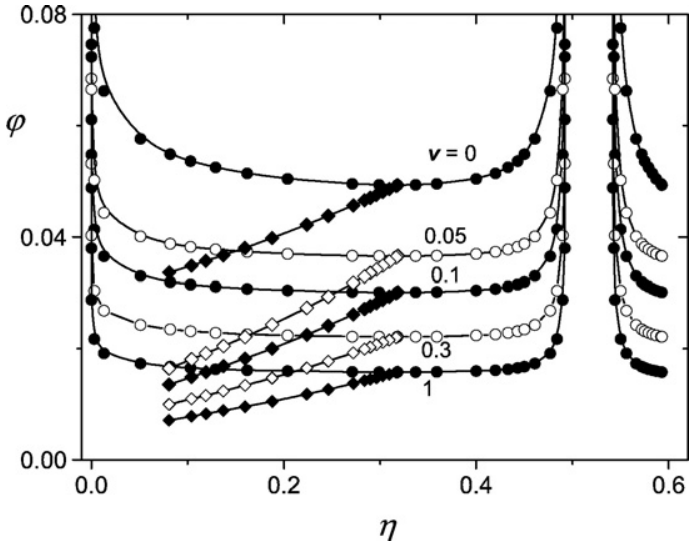

Fig. 5.9. Triple points (circles) and critical points (diamonds) for $a=30$ and five solvencies (the same set as in Fig. 5.7). The set of $q$ 's is again the standard set. The $\varphi^{*}$ values in the cep (Eq. (5.15a)) are $0.049(\boldsymbol{v}=0), 0.037,0.030,0.022$, and $0.016(\boldsymbol{v}=1)$. The $R^{*}$ values (Eq. (5.15b)) are 9.3 for $\boldsymbol{v}=0$ and 9.9 for $\boldsymbol{v}>0$. The corresponding $N^{*}$ values (Eq. (5.15c)) are $522(\boldsymbol{v}=0), 389,320,235$, and $168(\boldsymbol{v}=1)$.

but that hardly matters since the result for a theta solvent would be qualitatively the same. The GL binodal shows the normal features of a skewed parabola (with an asymptotic exclusion-type behavior for the $\mathrm{G}$ branch), with its minimum in the critical point and its ends at the fluid compositions of the triple point. The FS binodal for $q^{*}$ is identical to that in Fig. 5.6 since in this case $\tilde{q}_{R}=1$ so Eq. (5.16b) reduces to Eq. (5.14b). The FS binodal for $q=0.2$ behaves quite differently: it lies below that for $q^{*}$ at the liquid side, but for smaller $\eta$ it crosses the $q^{*}$ binodal and at the gas side it lies above that for $q^{*}$.

This inversion may be explained using the exclusion limit discussed in Section 3.2.1. For sufficiently low $\eta$, the gas branch follows Eq. (3.14): $\ln \eta \approx \ln 6.27+3 \ln (\Pi v)+\Pi v\left[1.35-(1+q)^{3}\right]$. For given (small) $\eta$ this gives a direct relation between $\Pi v$ and $q$. This relation may be rather accurately approximated as a power law: $\Pi v \sim q^{-2.8} \sim q_{R}^{-2.46}$ (we do not specify the numerical prefactor, which depends on the chosen value of $\eta$ ). Hence, $\Pi v$ increases strongly with decreasing $q$, as was shown in Fig. 3.1. When we convert to $y\left(y=q_{R}^{3} \Pi v\right.$ according to Eq. (5.8)) we find $y \sim q_{R}^{0.54}$ for the gas branches of the FS binodal. When in the next step we convert to $\varphi$, we find for constant $N$ $\left(\tilde{\varphi}=\tilde{y}\right.$, Eq. (5.14)) $\varphi \sim q_{R}^{0.54}$ : as $q$ decreases the gas branch of the FS binodal goes down, as shown in Fig. 5.6. However, for constant $a$ we have $\tilde{\varphi}=\tilde{y} \tilde{q}_{R}^{-4 / 3}$ (Eq. (5.16)), so $\varphi \sim q_{R}^{-0.79}$. The sign of the exponent explains the inversion found in the GS fluid branches in Fig. 5.8, with at low $\eta$ now a higher $\varphi$ for lower $q$.

Again we may convert the normalized plot $\tilde{\varphi}(\eta)$ of Fig. 5.8 into a non-normalized plot when we specify $a$ and $\boldsymbol{v}$; we then calculate $\varphi^{*}$ from Eq. (5.15a) and find $\varphi$ by multiplying $\tilde{\varphi}$ by $\varphi^{*}$. Fig. 5.9 shows such critical and triple points $\varphi(\eta)$ for $a=30$, for the same five solvencies as in Fig. 5.7. The values of $\varphi^{*}$ at the cep and the corresponding $R^{*}$ and $N^{*}$, calculated from Eq. $(5.15 b, c)$, are indicated in the legend. The general features of the triple curves are similar to those in Fig. 5.7, although the dependence of $\varphi^{t}$ on $q$ is weaker in Fig. 5.9, as discussed above. The critical curves go down in Fig. 5.9 and up in Fig. 5.7; also this behavior was explained above. For constant $a$, a stable colloidal liquid exists only when $R>R^{*}$ and $N>N^{*}$. In this example $(a=30) R^{*}$ is roughly constant (around 10) but the corresponding $N^{*}$ varies rather strongly with $\boldsymbol{v}$ : the maximum value is 522 for a theta solvent, but considerably lower in a good solvent ( 168 for $\boldsymbol{v}=1$ ). In a poorer solvent we need higher polymer chain lengths for a stable colloidal liquid.

\subsection{Phase diagrams in terms of the internal concentration $\phi$}

In the previous sections we considered only the polymer concentration $\varphi$ in the external reservoir. That is a transparent concept in the theoretical model, but $\varphi$ cannot be measured experimentally. An 
experimentalist has only access to the internal concentration $\phi=\alpha \varphi$, where $\alpha$ is the exclusion factor discussed in Section 2.2: it depends on the size ratio $q$ and the colloid concentration $\eta$. The dependence of $\alpha$ on $q$ and $\eta$ is monotonic, with $\alpha$ decreasing with increasing $q$ (at constant $\eta$ ) and with increasing $\eta$ (at constant $q$ ), see Fig. 2.2.

\subsubsection{The free volume fraction $\alpha$}

From the known colloid concentration $\eta$ in the critical endpoint (cep) and in the critical points (cp), triple points (tp), and along the binodals, it is straightforward to compute the free volume fraction $\alpha$, which depends only on $\eta$ and $q$, see Eq. (2.5). It turns out that we can also find rather accurate analytical simplifications for $\alpha$ in the various phases.

We start again with the cep, where $q^{*}=0.3275, \eta^{*}=0.3185$, and $\eta_{s}^{*}=0.5929$ have been found in Eq. (3.7). This immediately gives $\alpha^{*}$ and $\alpha_{s}^{*}$ for the fluid and solid parts of the cep:

$\alpha^{*}=0.3171 \quad \alpha_{s}^{*}=0.01199$

Hence, in the fluid part of the cep $32 \%$ of the volume is available for the polymer, whereas in the coexisting concentrated solid this is only $1.2 \%$.

Next we consider how $\alpha$ varies along the critical and triple curves. Numerical data for $\alpha^{c}\left(\eta^{c}\right)$ (diamonds) and $\alpha^{t}\left(\eta^{t}\right)$ (open circles) are shown in Fig. 5.10. The curves in this figure are not just curves connecting the data points, but are analytical approximations as discussed below (Eqs. (5.18)-(5.21)).

It is clear from Fig. 5.10 that $\alpha^{c}$ is nearly constant over the whole range of $\eta^{c}$; it varies from $\alpha^{*}=0.32$ at $\eta^{*}$ to $\alpha=0.36$ at low $\eta^{c}$. This can be explained by two compensating effects. Increasing the size ratio $q$ decreases the free volume fraction at fixed colloid concentration. Decreasing the colloid volume fraction, however, increases the free volume fraction. Apparently, the decrease in $\eta^{c}$ roughly compensates the increase of $q$, and the free volume fraction $\alpha^{c}$ remains almost constant.

The triple curves $\alpha^{t}$ consist again of three branches. In the gas branch $\alpha_{g}^{t}$ decreases more or less linearly from unity at small $\eta$ to $\alpha^{*}$ at $\eta^{*}=0.319$. The liquid branch $\alpha_{l}^{t}$ shows a linear decrease from $\alpha^{*}$ at $\eta^{*}$ to (nearly) zero at $\eta_{f}^{0}=0.492$. In the solid branch $\alpha_{s}^{t}$ increases from zero at $\eta_{s}^{0}=0.542$ to $\alpha_{s}^{*}$ at $\eta_{s}^{*}=0.593$. The liquid and solid branches of $\alpha^{t}$ are expanded in Fig. 5.10 as shown by the filled circles: these give the normalized quantities $\tilde{\alpha}_{g}^{t}=\alpha_{l}^{t} / \alpha^{*}$ and $\tilde{\alpha}_{l}^{t}=\alpha_{s}^{t} / \alpha_{s}^{*}$, which run from 0 to 1 . The fact that $\tilde{\alpha}_{s}^{t}$ increases with $\eta_{s}$ is because $q$ decreases (from infinity at $\eta_{s}^{0}$ to $q^{*}$ at $\eta^{*}$ ): this decreasing $q$ (which increases $\alpha$ )

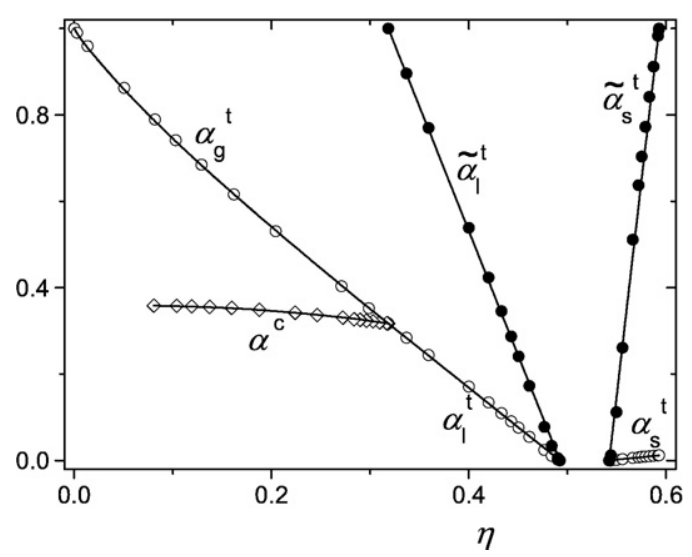

Fig. 5.10. The free volume fraction $\alpha^{t}$ for triple points (tp, open circles) and $\alpha^{c}$ for critical points (cp, diamonds) as a function of the corresponding colloid volume fraction $\eta^{t}$ and $\eta^{c}$. Symbols are the numerical data for the standard set of $q$ 's (Table 5.1). Curves are analytical according to Eqs. (5.18)-(5.21), using the numerical data for the $\eta$ 's. The closed symbols and corresponding lines give the normalized quantities $\tilde{\alpha}_{l}^{t}=\alpha_{l}^{t} / \alpha^{*}$ and $\tilde{\alpha}_{s}^{t}=\alpha_{s}^{t} / \alpha_{s}^{*}$. overcompensates the decrease in $\alpha$ due to an increasing $\eta$ in this solid branch.

For finding analytical approximations $\alpha(\eta)$ we start with the triple curves. For the liquid and solid branches $\alpha_{l}^{t}$ and $\alpha_{s}^{t}$ are well described by a straight line. Also $\alpha_{g}^{t}$ is more or less linear in $\eta_{g}^{t}$; upon closer inspection a linearity in $\left(\eta_{g}^{t}\right)^{0.85}$ is more accurate for the gas branch. Hence, we use the following analytical approximations:

$$
\begin{aligned}
& \alpha_{g}^{t}=1-\left(1-\alpha^{*}\right)\left(\tilde{\eta}_{g}^{t}\right)^{0.85}=1-1.806\left(\eta_{g}^{t}\right)^{0.85} \\
& \alpha_{l}^{t}=\alpha^{*} \frac{\eta_{f}^{0}-\eta_{l}^{t}}{\eta_{f}^{0}-\eta^{*}}=1.824\left(0.492-\eta_{l}^{t}\right) \\
& \alpha_{s}^{t}=\alpha_{s}^{*} \frac{\eta_{s}^{t}-\eta_{s}^{0}}{\eta_{s}^{*}-\eta_{s}^{0}}=0.237\left(\eta_{s}^{t}-0.542\right) .
\end{aligned}
$$

The triple curves in Fig. 5.10 are drawn according to Eqs. (5.18)(5.20). Eq. (5.18) is very accurate indeed: the largest deviation with the numerical data (for $q=0.45, \eta_{g}^{t}=0.013$ ) is about $0.5 \%$. Eq. (5.19) is of the same quality up to $\eta_{l}^{t}=0.46$; close to $\eta_{f}^{0}=0.492$ (where $\alpha$ is very small) it overestimates the numerical values. For Eq. (5.20) the absolute differences with the numerical values are small, but because $\alpha_{s}^{t}$ is small the relative error is somewhat higher than in Eqs. (5.18) and (5.19), up to several \% for $\eta_{s}^{t}$ above 0.566 , and more for $\eta_{s}^{t}$ close to $\eta_{s}^{0}=0.542$. Nevertheless, Eqs. (5.18)-(5.20) provide a very good estimate of the three branches of $\alpha^{t}$ so that it is easy to convert the external $\varphi$ 's (for which we also have analytical approximations) to internal $\phi$ 's.

The next issue is $\alpha^{c}$ along the critical curve. At first sight it is surprising that $\alpha^{c}$ is so insensitive to $\eta^{c}$ (or, for that matter, to $q$ ). The value at the cep is $\alpha^{c}=\alpha^{*}=0.317$. We can derive the high- $q\left(\right.$ low $\left.-\eta^{c}\right)$ limit from Eq. (2.5), which reduces to $\alpha=\beta=e^{-A f}$ in this limit; as before $A=(1+q)^{3}-1$. Then Eq. (3.5) simplifies to $1 / f^{c}-A=0$, or $A f^{c}=1$. This implies that the power law $f^{c} \sim q^{-1.2}$ (Eq. (3.18)) no longer holds for (very) high $q$, then $\eta^{c}=f^{c}=1 / A$ is more appropriate. Moncho-Jorda et al. [28] derived - in a quite different way $-\eta^{c}=(1+q)^{-3}$ for high $q$, which is essentially the same result as our $\eta^{c}=1 / A$ in this limit.

With $\alpha^{c}=e^{-A f^{c}}=e^{-1}=0.368$ for high $q$ and $\alpha^{c}=0.317$ for $q^{*}$, we can understand that $\alpha^{c}$ hardly varies along the critical curve. In a simplistic approach we could assume a linear behavior $\alpha^{c}=\alpha^{e}-\left(\alpha^{e}-\alpha^{*}\right) \tilde{\eta}^{c}$ between the limits $\alpha^{e}$ and $\alpha^{*}$. In Fig. 5.10 we see that such a linear behavior is not quite adequate. When we enlarge the numerical plot $\alpha^{c}$ $\left(\eta^{c}\right)$ (not shown) it turns out that a linearity in $\left(\tilde{\eta}^{c}\right)^{2.5}$ is very accurate:

$\alpha^{c}=\alpha^{e}-\left(\alpha^{e}-\alpha^{*}\right)\left(\tilde{\eta}^{c}\right)^{2.5}=0.36\left(1-0.119\left(\tilde{\eta}^{c}\right)^{2.5}\right)$.

Although the theoretical limit for $\alpha^{e}$ is 0.368 (for very high $q$ ), for the $q$ range shown in Fig. 5.10 (up to $q=1.2$ ) a slightly smaller value works better; we used 0.36 . With this value of $\alpha^{e}$, the deviation of Eq. (5.21) from the numerical values is less than $0.2 \%$ over the range $q^{*}<q<1.2$.

Since we have now analytical expressions for $\alpha(\eta)$, we can immediately find the dependence $\alpha(q)$ by substituting the analytical dependences $\eta(q)$. These are obtained from $f^{c}(q)$ (Eq. (3.18)), $f_{s}^{t}(q)$ (Eq. (3.20)), $f_{l}^{t}(q)$ (Eq. (3.22)), and $f_{g}^{t}(q)$ (Eq. (3.24)), by converting $f$ to $\eta=f /(1+f)$. This substitution is straightforward, and there is no need to give the explicit expressions.

The curves in Fig. 5.11 gives results $\alpha(q)$ obtained by using these analytical $\eta$ 's, in a normalized form $\tilde{\alpha}$ as a function of $1 / \tilde{q}=q^{*} / q$. Only for $\alpha_{g}^{t}$ (which becomes unity for high $q$ ) did we plot the nonnormalized version. For $\tilde{\alpha}^{c}$ and $\tilde{\alpha}_{l}^{t}$ we normalized on $\alpha^{*}$, for $\tilde{\alpha}_{s}^{t}$ on $\alpha_{s}^{*}$. For most of the curves in Fig. 5.11 the agreement with the numerical data (symbols) is nearly as good as in Fig. 5.10 (where numerical $\eta$ 's were used). However, for $\tilde{\alpha}_{s}^{t}$ the approximation is slightly less accurate: the dashed curve (Eq. (5.20) with numerical $\eta$ 's) is 


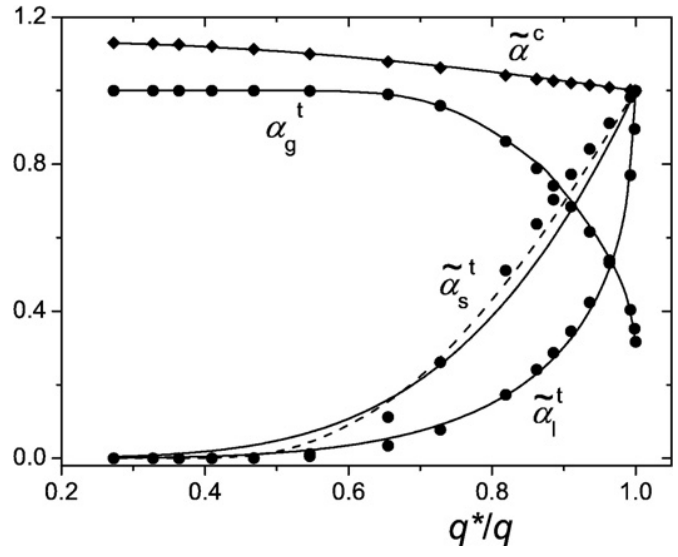

Fig. 5.11. Variation of $\alpha^{t}$ for triple points (tp, three branches) and of $\alpha^{c}$ for critical points (cp) with $1 / q \equiv q^{*} / q$. The gas branch $\alpha_{\mathrm{g}}^{t}$ of the triple curve is not normalized (so it is $\alpha^{*}$ in the cep), the other quantities are normalized on the cep. Symbols (circles for tp, diamonds for $\mathrm{cp}$ ) are the numerical data for the standard set of $q$ 's (Table 5.1). Solid curves are analytical according to Eqs. (5.18)-(5.21), with the analytical results (Eqs. (3.18), (3.20), (3.22), (3.24)) for the $\eta$ 's. The dashed curve for $\tilde{\alpha}_{s}^{t}$ is Eq. (5.20) with numerical $\eta$ 's.

somewhat better. In both cases the absolute errors are small, as $\alpha_{s}^{t}$ is small.

\subsubsection{Critical and triple points in internal concentrations}

In Fig. 5.2 we plotted $\tilde{y}^{c}$ and $\tilde{y}^{t}$ as a function of $\tilde{q}_{R}$. For the case of constant $N$, these quantities are equal to the normalized external concentrations $\tilde{\varphi}^{c}$ and $\tilde{\varphi}^{t}$, respectively. In terms of the external concentration the triple curve is a single curve, as $\varphi$ determines the chemical potential of the polymer, which is the same in the three coexisting phases. For the internal concentrations $\phi^{t}=\alpha \varphi^{t}$ the triple curve falls apart in three branches because $\alpha_{g}^{t}, \alpha_{l}^{t}$, and $\alpha_{s}^{t}$ outside the cep are (very) different. Fig. 5.12 gives $\phi^{c}$ and the three branches of $\phi^{t}$ (normalized on $\phi^{*}=\alpha^{*} \varphi^{*}$ in the cep) as a function of $\tilde{q}_{R}$ on a double-logarithmic scale. Circles are numerical triple points, diamonds are numerical critical points; the curves are analytical. Filled symbols and solid curves are for the case of constant chain length $N$ (where $\tilde{\phi}=\tilde{\alpha} \tilde{y}$ ), open symbols with dashed curves are for constant particle radius $a$ (where $\tilde{\phi}=\tilde{\alpha} \tilde{y} \tilde{q}_{R}^{-4 / 3}$ ). The figure is for a good solvent, but a similar plot for a theta solvent is nearly the same (with a small difference for the constant- $a$ curves because then $\tilde{\phi}=\tilde{\alpha} \tilde{y} \tilde{q}_{R}^{-1}$ ).

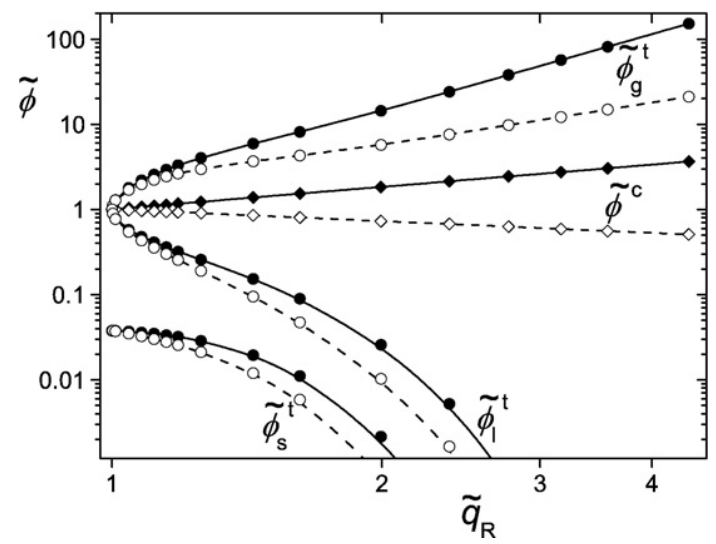

Fig. 5.12. Normalized internal concentrations $\tilde{\phi}^{c}$ (cp, diamonds) and $\tilde{\phi}^{t}$ (tp, circles) as a function of $\tilde{q}_{R}$ for a good solvent, on a double-logarithmic scale. Closed symbols and solid curves are for constant $N$, open symbols with dashed curves are for constant a; the symbols correspond to the standard set of $q$ 's (Table 5.1). The normalized $\tilde{\phi}$-values may be converted to real concentrations $\phi$ by multiplying with $\phi^{*}=\alpha^{*} \varphi^{*}$, where $\varphi^{*}$ is given by Eq. (5.13a) (constant $N$ ) or Eq. (5.15a) (constant $a$ ).
We first discuss the curves for constant $N$ (filled symbols, solid curves). The critical curve is approximately the same as in Fig. 5.2 because $\alpha^{c}$ is nearly constant over the entire $q$ range; the scaling is $\phi^{c} \sim q_{R}^{0.72}$ (Eq. (5.10)) so $\phi^{c}$ increases with $q_{R}$. The high- $q$ part of $\tilde{\phi}_{g}^{t}$ (where $\left.\alpha_{g}^{t}=1\right)$ is also the same as $\tilde{y}^{t}$ in Fig. 5.2, apart from a shift $-\log \alpha^{*}=0.5$ upwards; the scaling is $\phi^{t} \sim q_{R}^{3}$. Closer to the cep the curve deviates from this scaling behavior for two reasons: $\tilde{y}^{t}$ deviates upwards and $\tilde{\alpha}_{g}^{t}$ bends downwards, and the second effect is stronger. The curves for $\tilde{\phi}_{l}^{t}$ and $\tilde{\phi}_{s}^{t}$ do not show a clear scaling behavior but both quantities become very small for $q>0.6$ where the exclusion limit applies.

The constant- $a$ results (open symbols connected by dashed lines) can be related to the constant- $N$ data taking into account the factor $\tilde{q}_{R}^{-4 / 3}$. Hence, the critical curve goes down as $\phi^{c} \sim q_{R}^{-0.61}$, as in Fig. 5.8. The high- $q$ gas branch of the triple curve scales as $\phi^{t} \sim q_{R}^{5 / 3}$, which is a weaker increase than for constant $N$. Due to the same factor $\tilde{\mathrm{q}}_{R}^{-4 / 3}$ the liquid and solid branches of the triple curve go down more steeply for constant $a$.

The normalized $\tilde{\phi}$ data of Fig. 5.12 may be converted to the real concentrations $\phi$ by multiplying, for given $\boldsymbol{v}$ and $N$ or $a$, with $\phi^{*}=\alpha^{*} \varphi^{*}=0.317 \varphi^{*}$, where $\varphi^{*}$ is given by Eq. (5.13a) (constant $N$ ) or Eq. (5.15a) (constant $a$ ). So we could make non-normalized plots of the type of Fig. 5.7 or 5.9 also for the internal polymer concentrations; we will not do that here.

\subsubsection{Phase diagrams at constant chain length $N$}

Fig. 5.13 shows a normalized $\tilde{\phi}(\eta)$ phase diagram for a good solvent and constant $N$, with three GL binodals ( $q=0.35,0.4,0.45$ ) and one FS binodal $(q=0.2)$. Only the three triple points (nine circles) and the three critical points (diamonds) corresponding to the GL binodals are indicated. The asterisk at $\tilde{\phi}=1, \eta=\eta^{*}$ is the cep. For $q=0.45$ we show also the triple triangle connecting the three $\tilde{\phi}^{t}, \eta^{t}$ pairs corresponding to this $q$ value.

Fig. 5.13 for $\tilde{\phi}(\eta)$ is essentially the same as Fig. 5.6 for $\tilde{\varphi}(\eta)$ : the only difference is that we multiplied $\tilde{\varphi}$ (Fig. 5.6) with $\tilde{\alpha}$ to obtain $\tilde{\phi}$ (Fig. 5.13). Nevertheless, the transparent Fig. 5.6 now transforms into a more complicated Fig. 5.13: in this figure the critical points, the (fluid parts of the) triple curve, and all the GL binodals more or less collapse on a single curve. The main difference between the GL binodals is their span: that for $q=0.35$ is short, that for $q=0.45$ is long, but the short one can hardly be distinguished from the middle part of the long binodal. We did not indicate in Fig. 5.13 the FS binodal for $q=q^{*}$ (which we did in Fig. 5.6): it nearly coincides with the GL binodals (and the curve connecting the triple points and that for the critical points).

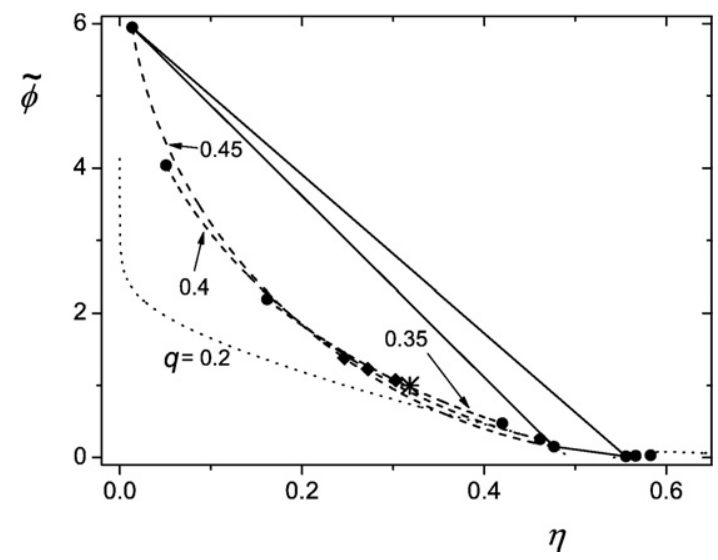

Fig. 5.13. Normalized phase diagram $\tilde{\phi}(\eta)$ for a constant chain length $N$ and a good solvent. Three GL binodals $(q=0.35,0.4,0.45)$ are indicated as the dashed curves. The corresponding triple points are the (nine) circles, the three critical points are the diamonds. The asterisk is the cep at $\tilde{\phi}^{*}=1, \eta=\eta^{*}$. The triple triangle connects the three $\tilde{\phi}^{t}, \eta^{t}$ pairs for $q=0.45$. The dotted curve is the FS binodal for $q=0.2$. 


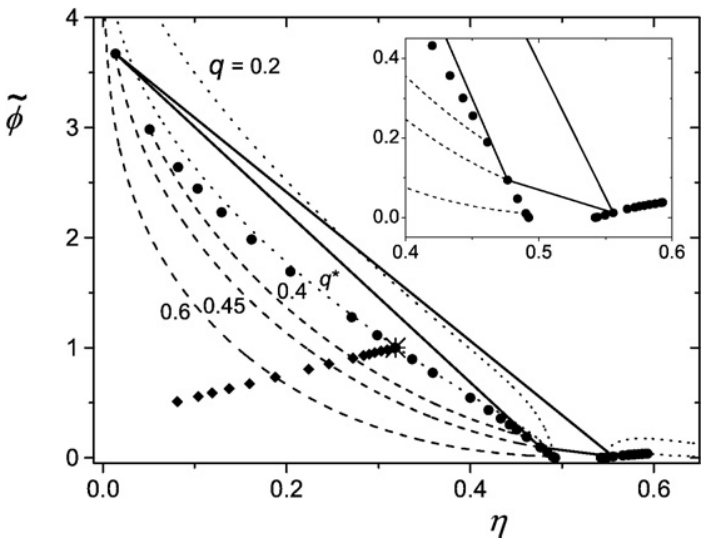

Fig. 5.14. Normalized phase diagram $\tilde{\phi}(\eta)$ for a constant particle radius $a$ and a good solvent. Three GL binodals $(q=0.4,0.45,0.6)$ are indicated as the dashed curves. Triple points (circles) and critical points (diamonds) are for the standard set (Table 5.1) for $q$; the highest triple point shown in the $\mathrm{G}$ branch is for $q=0.45$. The asterisk is the cep at $\tilde{\phi}^{*}=1, \eta=\eta^{*}$. The triple triangle connecting the three $\left(\tilde{\phi}^{t}, \eta^{t}\right)$ pairs is for $q=0.45$. The dotted curves are the FS binodals for $q=q^{*}$ and 0.2 . The inset shows more detail for high $\eta$; here we omitted the FS binodals.

\subsubsection{Phase diagrams at constant particle radius a}

Fig. 5.14 demonstrates the essential features of a $\tilde{\phi}(\eta)$ diagram at constant $a$, again for a good solvent. This figure may be compared with the $\tilde{\varphi}(\eta)$ diagram in Fig. 5.8 for the same conditions. Now the triple points (circles) and the critical points (diamonds) can be clearly distinguished, unlike in Fig. 5.13 where critical and triple curves more or less coincide. In Fig. 5.14 we show critical and triple points for the standard $q$ set (Table 5.1); for the $G$ branch the highest triple point visible is for $q=0.45$. The asterisk is again the cep. Three GL binodals (dashed, $q=0.4,0.45,0.6$ ) and two FS binodals (dotted, $q=q^{*}$ and 0.2 ) are shown. The FS binodal for $q^{*}$ passes through the cep and is rather close (but above) the triple curve outside the cep. The binodal for $q=0.2$ is situated at (much) higher $\phi$. Fig. 5.14 gives also the triple triangle for $q=0.45$.

The inset of Fig. 5.14 gives more detail of the concentrated region $0.4<\eta<0.6$. Triple points ( $\mathrm{L}$ and $\mathrm{S}$ branches) are again the circles; those on the $\mathrm{L}$ branch are for $q=0.35$ and up. The end parts of the three GL binodals (L branch) are visible (dotted). Also the lower end of the triple triangle is indicated. For the sake of clarity we omitted the FS binodals in the inset.

In Figs. 5.13 and 5.14 we gave only good-solvent versions, but those for a theta solvent would be essentially the same. The normalized $\tilde{\phi}$ data in these figures can be converted to real concentrations $\phi$ by multiplying with $\phi^{*}=\alpha^{*} \varphi^{*}$, where $\varphi^{*}$ is given by Eq. (5.13a) (constant $N$ ) or Eq. (5.15a) (constant $a$ ); to that end we have to specify $\boldsymbol{v}$ and $N$ or a. Such a conversion does not change the general features of the phase diagrams.

\section{Phase diagrams for 'variable $q$ '}

In this chapter we introduce our new model where $q$ is allowed to vary with polymer concentration, hence the indication 'variable $q$ '. We now account for the crossover in the length scale $\delta_{\mathrm{p}}$ (depletion thickness next to a plate) from the constant value $\delta_{0}$ in dilute polymer solutions to the concentration-dependent correlation length (blob size) $\xi$ in semidilute polymer solutions. This is accomplished through the combination rule $\delta_{\mathrm{p}}^{-2}=\delta_{0}^{-2}+\xi^{-2}$ derived before $[45,46]$. Moreover, we incorporate non-ideal contributions to the osmotic pressure $\Pi$, which has the limits $\Pi_{0}=\varphi / N$ (dilute) and $\Pi_{\mathrm{sd}} \sim \xi^{-3}$ (semidilute). Here a simple additivity $\Pi=\Pi_{0}+\Pi_{\mathrm{sd}}$ is adequate [46]. For both $\delta$ and $\Pi$ the semidilute scaling exponents are different for a theta solvent and a good solvent. As in the previous chapters we use the two-letter abbreviations $\mathrm{mf}$ and ev to distinguish between these two cases.
6.1. Concentration dependence of depletion thickness and osmotic pressure

\subsubsection{Depletion thickness in flat geometry}

In this section we consider the depletion thickness $\delta_{\mathrm{p}}$ next to a plate (curvature effects are accounted for in Section 6.2). The dilute limit for $\delta_{\mathrm{p}}$ is $\delta_{0}=p R$ as given in Eq. (5.1): $\delta_{0} \sim N^{v}$ depends on chain length (and solvency) but not on polymer concentration. For semidilute solutions De Gennes [60] derived $\delta_{\mathrm{p}}=\xi$, where the blob size (correlation length) $\xi$ is only a function of the polymer concentration $\varphi$ and does not depend on $N$ :

$\xi \sim \varphi^{-\gamma} \quad \gamma=\left\{\begin{array}{ll}1 & \mathrm{mf} \\ 0.77 & \mathrm{ev}\end{array}\right.$.

Hence, with increasing $\varphi$ the relevant length scale decreases from $R$ in dilute solutions to the much smaller value $\xi$ in semidilute solutions. In Eq. (6.1) we replaced the classical De Gennes exponent $\gamma=3 / 4$ for excluded-volume chains by the more accurate renormalization-group (RG) result $\gamma=0.77$ [44,61]. The exponent $\gamma$ is directly coupled to the Flory exponent $\nu$ (which is $3 / 5$ in the classical picture and 0.588 in RG):

$1 / \gamma+1 / v=3$

When we take $\gamma=0.77$ for ev, we should for consistency also modify the scaling exponent in $\varphi_{\mathrm{ov}}: \varphi_{\mathrm{ov}} \sim N R^{-3} \sim R^{1 / v-3}=R^{-1 / \gamma}$ according to Eq. (4.6). In Eq. (4.8) we used $\gamma=3 / 4$ and $\varphi_{\mathrm{ov}} \sim R^{-4 / 3}$ for ev. The more accurate version of Eq. (4.8) is

$\varphi_{\mathrm{ov}} \sim R^{-1 / \gamma} \quad \varphi_{\mathrm{ov}}= \begin{cases}(9 / 2 \pi) R^{-1} & \mathrm{mf} \\ 0.87 v^{-0.28} R^{-1.3} & \mathrm{ev}\end{cases}$

whereby we assume that the numerical prefactor in Eq. (4.8) still applies.

The first task is to find the prefactor in Eq. (6.1). For $\mathrm{mf}$ this is known analytically $[45,46]$ : $\xi=b \varphi^{-1}$, where $b=\operatorname{atanh}(1 / \sqrt{3})=0.658$. We shall need primarily the ratio $\xi / \delta_{0}$ between the semidilute and dilute limits. With $\delta_{0}^{2}=(4 / \pi) R^{2}$ (Eq. (5.1)) we find $\left(\delta_{0} / \xi\right)^{2}=\left(4 b^{-2} / \pi\right)$ $R^{2} \varphi^{2}=\left(4 \mathrm{~b}^{-2} / \pi\right)\left(R \varphi_{\mathrm{ov}}\right)^{2} y^{2}$, where $R \varphi_{\mathrm{ov}}=9 / 2 \pi$ according to Eq. (6.3). Hence $\left(\delta_{0} / \xi\right)^{2}=6.02 y^{2}$ for mf. For ev there are accurate computer simulation results [38]: $\xi / \delta_{0}=0.503 y^{-0.77}$. We thus have the following semidilute limits for $\delta_{\mathrm{p}}=\xi$ :

$\left(\frac{\delta_{0}}{\xi}\right)^{2}=\left\{\begin{array}{ll}6.02 y^{2} & \mathrm{mf} \\ 3.95 y^{1.54} & \mathrm{ev}\end{array} \quad\left(\frac{\delta_{0}}{\xi}\right)^{2}=c_{1} y^{2 \gamma}\right.$.

Previously $[45,46]$ we have shown that the crossover between the dilute and semidilute limits is accurately described by

$\delta_{\mathrm{p}}^{-2}=\delta_{0}^{-2}+\xi^{-2} \quad\left(\delta_{0} / \delta_{\mathrm{p}}\right)^{2}=1+\left(\delta_{0} / \xi\right)^{2}$.

Inserting Eq. (6.4) into Eq. (6.5) we find for the concentration dependence of the depletion thickness $\delta_{\mathrm{p}}$ the following simple relation:

$\left(\delta_{0} / \delta_{\mathrm{p}}\right)^{2}=1+c_{1} y^{2 \gamma} \quad c_{1}= \begin{cases}6.02 & \mathrm{mf} \\ 3.95 & \mathrm{ev}\end{cases}$

This relation is accurate for any concentration up to and including the semidilute concentration regime. It incorporates the dilute limit $\delta_{\mathrm{p}}=\delta_{0}$ for $y \rightarrow 0$ and the semidilute limit $\delta_{\mathrm{p}} / \delta_{0} \sim y^{-\gamma}$, but describes the crossover as well. The ratio $\delta_{\mathrm{p}} / \delta_{0}$ depends only on the reduced concentration $y=\varphi / \varphi_{\text {ov }}$.

For applying Eq. (6.6) to polymer/colloid mixtures, we have to compare $\delta_{\mathrm{p}}$ to the particle radius $a$. Analogously to Eq. (5.2) we define the size ratio $q_{\mathrm{p}}$ as:

$q_{\mathrm{p}}=\delta_{\mathrm{p}} / a$ 
Since $q_{0}=\delta_{0} / a=p q_{R}$ (Eq. (5.2)) we may replace in Eq. (6.6) the ratio $\delta_{0} / \delta_{\mathrm{p}}$ by $q_{\mathrm{o}} / q_{\mathrm{p}}=p q_{R} / q_{\mathrm{p}}$ (see also Eq. (6.16)).

\subsubsection{Osmotic pressure}

In the dilute limit we have the Van 't Hoff law $\Pi=\Pi_{0}=\varphi / N$, which may be written in different ways:

$\Pi_{0}=\frac{\varphi}{N}=y \frac{\varphi_{\mathrm{ov}}}{N}=\frac{y}{V_{\text {coil }}} \quad \Pi_{0} v=y q_{R}^{-3}$.

In Eq. (6.8a) we inserted $\varphi=y \varphi_{\text {ov }}=y N / V_{\text {coil }}$ (Eq. (4.6)), in Eq. (6.8b) we used $V_{\text {coil }} / v=(R / a)^{3}=q_{R}^{3}$.

For the semidilute limit we start from the well-known scaling result [60]:

$\Pi_{\mathrm{sd}} \sim \xi^{-3} \quad \Pi_{\mathrm{sd}} \sim \varphi^{3 \gamma}$.

We need also the numerical prefactors in Eq. (6.9), and again we are primarily interested in the ratio $\Pi_{\mathrm{sd}} / \Pi_{0}$ between the semidilute and dilute limits. For $\mathrm{mf}$ the Flory-Huggins expression $\Pi=\varphi / N-\ln (1-\varphi)-$ $\varphi-\varphi^{2} / 2$ [57] for a theta solvent is a good starting point. Expanding the logarithm up to third order we find the simple additivity $\Pi=\Pi_{0}+\Pi_{\mathrm{sd}}$, where $\Pi_{0}=\varphi / N$ Eq. (6.8a) and $\Pi_{\mathrm{sd}}=\varphi^{3} / 3$. The prefactor in Eq. (6.9b) is thus $1 / 3$. The ratio $\Pi_{\mathrm{sd}} / \Pi_{0}$ follows as $N \varphi^{2} / 3=2 R^{2} \varphi^{2}$ where $R=(9 / 2 \pi)$ $\varphi_{\mathrm{ov}}^{-1}$ (Eq. (6.3)). Hence, $\Pi_{\mathrm{sd}} / \Pi_{0}=4.10 y^{2}$ in $\mathrm{mf}$. For ev we can use the RG result $\Pi_{\mathrm{sd}} / \Pi_{0}=1.62 y^{1.31}$ [44], where the exponent is $3 \gamma-1$. Hence

$\frac{\Pi_{\mathrm{sd}}}{\Pi_{0}}=\left\{\begin{array}{lll}4.10 y^{2} & \mathrm{mf} & \Pi_{\mathrm{sd}} \\ 1.62 y^{1.31} & \mathrm{ev} & \Pi_{0} y^{3 \gamma-1} .\end{array}\right.$

We saw above that the Flory-Huggins expression for mf leads to $\Pi=\Pi_{0}+\Pi_{\mathrm{sd}}$. The same additivity rule is a very good approximation for ev as well [46]. Therefore the concentration dependence of $\Pi$ can be described as

$\Pi=\Pi_{0}+\Pi_{\mathrm{sd}} \quad \Pi / \Pi_{0}=1+\Pi_{\mathrm{sd}} / \Pi_{0}$.

Inserting Eq. (6.10) into Eq. (6.11) and substituting $\Pi_{0} v=y q_{R}^{-3}$ (Eq. (6.8)) we find

$\Pi v=q_{R}^{-3}\left[y+c_{2} y^{3 \gamma}\right] \quad c_{2}=\left\{\begin{array}{ll}4.10 & \mathrm{mf} \\ 1.62 & \mathrm{ev}\end{array}\right.$.

Again we recover the correct dilute and semidilute limits. Eqs. (6.6) for $\delta_{\mathrm{p}}$ and (6.12) for $\Pi v$ constitute the basis for our 'variable $q$ ' model.

\subsubsection{The protein limit}

In this section we discuss some general trends for the protein limit, which follow automatically from our generalized treatment by omitting the unity term in Eq. (6.6) and the ideal term in Eq. (6.12).

When the polymer concentrations are in the semidilute regime the ratio $\xi / \delta_{0}$ is a simple scaling function of $y=\varphi / \varphi_{\text {ov. }}$. We rewrite Eq. (6.4) as

$\xi \sim \delta_{0} y^{-\gamma} \quad q_{\mathrm{sd}} \sim q_{0} y^{-\gamma}$

where $q_{\mathrm{sd}}$ is the semidilute limit of $q_{\mathrm{p}}$. The numerical prefactors are also known, but we do not need those in the present section. What we do need is the fact that $\xi$ is independent of $R \approx \delta_{0}: \delta_{0} y^{-\gamma}$ is independent of $\delta_{0}$ so $\delta_{0} y^{-\gamma}$ is constant or $y \sim \delta_{0}^{1 / \gamma}$. Similarly, $q_{\text {sd }}$ is independent of $q_{0}$ (or $q_{R}$ ). We may thus write

$y \sim q_{0}^{1 / \gamma} \sim q_{R}^{1 / \gamma}$.

Applying this equation to critical and triple points, we expect that $y^{c}$ and $y^{t}$ in the protein limit are proportional to $q_{R}^{1 / \gamma}$. We will see that the numerical results confirm this scaling result.

Another way of interpreting Eq. (6.14) is that $y q_{R}^{-1 / \gamma}$ should be independent of $q_{R}$. We shall find in the next sections that indeed the protein-limit binodals for various $q_{R}$ collapse on a single curve when $y q_{R}^{-1 / \gamma}$ is plotted against the colloid concentration $\eta$.
Not only $q_{\mathrm{sd}}$ and $y q_{R}^{-1 / \gamma}$ become independent of $q_{R}$ in the protein limit, but also $\Pi v \sim q_{R}^{-3} y^{3 \gamma}=\left[y q_{R}^{-1 / \gamma}\right]^{3 \gamma}$ (Eq. (6.12)). We will see later that also the colloid concentrations $\eta$ and the free volume fractions $\alpha$ along the binodals become independent of the size ratio $q_{R}=R / a$.

\subsection{The parameters $Y$ and $q$}

In the previous section we saw that the parameter combination $\mathrm{y} q_{R}^{-1 / \gamma}$ becomes constant in the protein limit. It is convenient to introduce a separate symbol for this combination:

$Y=y q_{R}^{-1 / \gamma}$

The value of $Y$ is constant in the protein limit (large $q_{R}$ ); we shall use the symbols $Y_{\infty}^{c}$ for the critical point and $Y_{\infty}{ }^{t}$ for the triple point in this limit. In the colloid limit $Y$ varies with $q_{R}$ but even in the cep $Y^{*}$ is not greatly different from the protein-limit values $Y_{\infty}^{c}$ and $Y_{\infty}^{t}$. In terms of the rescaled polymer concentration $Y$, GL binodal concentrations are always of order unity.

It is then useful to rewrite Eq. (6.6) for $\delta_{0} / \delta_{\mathrm{p}}=q_{\mathrm{o}} / q_{\mathrm{p}}=p q_{R} / q_{\mathrm{p}}$ in terms of $q_{R}$ and $Y$ :

$q_{\mathrm{p}}^{-2}=p^{-2}\left[q_{R}^{-2}+c_{1} Y^{2 \gamma}\right] \quad q_{\mathrm{p}}=\frac{p}{\sqrt{q_{R}^{-2}+c_{1} Y^{2 \gamma}}}$

The two limits are immediately clear. In the colloid limit $(Y \rightarrow 0)$ we have $q_{\mathrm{p}}=q_{0}=p q_{R}$, in the protein limit $q_{\mathrm{p}}=\left(p / \sqrt{c_{1}}\right) Y^{-\gamma}$. Since $Y=Y_{\infty}$ does not depend on $q_{R}$ in the protein limit, $q_{\mathrm{p}}=q_{\mathrm{sd}}$ attains a constant value as well.

For describing the phase behavior we have to include curvature effects, whereby we convert $\delta_{\mathrm{p}}$ (plate) to $\delta$ (sphere) or, equivalently, $q_{\mathrm{p}}$ to $q$. We assume that the curvature dependence can be incorporated through Eq. (5.5) by replacing the concentration-independent parameter $\mathrm{q}_{\mathrm{o}}$ for flat geometry by the concentration-dependent $q_{\mathrm{p}}$ :

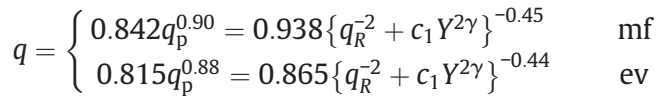

where we substitute $c_{1}=6.02$ and $\gamma=1$ for $\mathrm{mf}$ and $c_{1}=3.95$ and $\gamma=0.77$ for ev. Also $q=\delta / a$ is only a function of $q_{R}$ and $Y$. In the colloid limit this equation reduces to Eq. (5.5); then $q=q(0)=0.938 q_{R}^{0.9}$ (mf) or $0.865 q_{R}^{0.88}(\mathrm{ev})$ is of order of (but not equal to) $q_{R}$. In the semidilute limit (i.e., the protein limit) $q$ becomes independent of $q_{R}$.

Fig. 6.1 gives a plot of $q(y) / q(0)$ as a function of $y=Y q_{R}^{1 / \gamma}$, for both $\mathrm{mf}$ (lower curves) and ev (top curves). This ratio may be written as $\left(1+c_{1} y^{2 \gamma}\right)^{-\tau}(\tau=0.45$ or 0.44$)$, which is unity for $y \rightarrow 0$. The

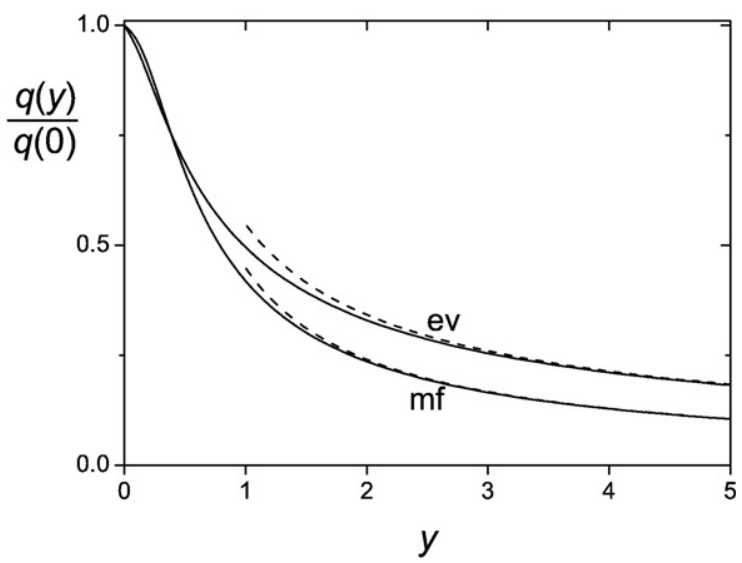

Fig. 6.1. The dependence $q(y) / q(0)$ on the reduced polymer concentration $y=\varphi / \varphi_{\text {ov }}$ Solid curves follow Eq. (6.17), dashed curves are the semidilute limit $q(y) / q(0)=$ $0.45 y^{-0.9}(\mathrm{mf})$ or $0.55 y^{-0.68}(\mathrm{ev})$; these limits are obtained by omitting the $q_{R}$ terms in Eq. (6.17). 
parameter $q(0)$ as a function of $q_{0}$ or $q_{R}=q_{0} / p$ is shown in Fig. 5.1; Fig. 6.1 adds the concentration dependence. In the region $0<y<1 q(y)$ decreases rather strongly; for high $y$ the semidilute power law $q(y) / q(0)=$ $0.45 y^{-0.9}(\mathrm{mf})$ or $0.55 y^{-0.68}(\mathrm{ev})$ applies. The dashed curves give these semidilute limits, which are adequate above (roughly) $y=1$.

We may also rewrite Eq. (6.12) in terms of $q_{R}$ and $Y$ :

$\Pi v=q_{R}^{-1 / v} Y+c_{2} Y^{3 \gamma}$

where $-1 / \nu=-3+1 / \gamma\left(\right.$ Eq. (6.2)) and $c_{2}=4.10(\mathrm{mf})$ or $1.62(\mathrm{ev})$. In the colloid limit we have $\Pi v=q_{R}^{-3+1 / \gamma} Y$ or $\Pi v=q_{R}^{-3} y$, which is Eq. (6.8), and in the protein limit $\Pi v=c_{2} Y^{3 \gamma}$, which is again independent of $q_{R}$.

The integrals of Section 2.3.3 for the polymer contribution to $\omega, \mu$, and $p v$ (and the derivatives of $p v$ ) were formulated in terms of the integration variable $\Pi v$. In these integrals the free volume fraction $\alpha$ and its derivatives occur; these functions depend on $q=q\left(q_{R}, Y\right)$. On account of Eqs. (6.17) and (6.18) it is now mathematically more convenient to replace the integration variable $\Pi v$ by $Y$, using $d \Pi v=$ $(\partial \Pi v / \partial Y) d Y$, where $\partial \Pi v / \partial Y$ is given by

$\frac{\partial \Pi v}{\partial Y}=q_{R}^{-1 / v}+3 \gamma c_{2} Y^{3 \gamma-1}$

This equation is used to calculate phase diagrams in terms of the variable $Y$.

\subsection{Critical endpoint}

The critical endpoint is calculated from the four equations of Eq. (2.28), using Eqs. (2.21)-(2.23) for $\mu^{p},(p v)^{p}$, and the derivatives of $(p v)^{p}$. In the integrals of Eqs. (2.21)-(2.23) we replace the integration variable $\Pi v$ by $Y$, using Eq. (6.19). The parameter $q$ occurring in the functions $g$ (for $\mu$ ), $h$ (for $p v$ ) and in the derivatives $\beta_{1}$ and $\beta_{2}$ (for the critical point) depends on $q_{R}$ and $Y$ according to Eq. (6.17). The four simultaneous equations determining the cep are then solved for the four parameters $q_{R}^{*}, Y^{*}, f^{*}$, and $f_{s}^{*}$. From these four, the auxiliary parameters $q^{*}, q_{0}^{*}, y^{*},(\Pi v)^{*}, \eta^{*}$, and $\eta_{s}^{*}$ follow directly. Because the constants in Eqs. (6.17) and (6.19) are different for $\mathrm{mf}$ and ev (see Eqs. (6.6) and (6.12)), we obtain slightly different pairs of parameters.

The $q$ parameters in the cep are found to be

$q^{*}=\left\{\begin{array}{l}0.290 \\ 0.273\end{array} \quad q_{0}^{*}=\left\{\begin{array}{l}0.381 \\ 0.416\end{array} \quad q_{R}^{*}=\left\{\begin{array}{cc}0.337 & \mathrm{mf} \\ 0.388 & \mathrm{ev}\end{array}\right.\right.\right.$.

These values may be compared with our previous result for 'fixed $q^{\prime}$ (Eq. (5.4)): $q^{*}=0.328, q_{0}^{*}=0.350(\mathrm{mf})$ or $0.353(\mathrm{ev}), q_{R}^{*}=0.310(\mathrm{mf})$ or $0.330(\mathrm{ev})$. The differences with Eq. (6.20) are small, which shows that in the cep the approximation of a constant $q, q \approx q(0)$ as made in chapter 5, is reasonable: in the cep the $Y$ terms in Eqs. (6.17) and (6.19) do not yet play an important role.

The results for $Y^{*}, y^{*}$ and $(\Pi v)^{*}$ in the cep are:

$Y^{*}=\left\{\begin{array}{l}0.899 \\ 1.464\end{array} \quad y^{*}=\left\{\begin{array}{l}0.304 \\ 0.428\end{array} \quad(\Pi v)^{*}=\left\{\begin{array}{ll}10.931 & \mathrm{mf} \\ 11.245 & \mathrm{ev}\end{array} \cdot(6.21)\right.\right.\right.$

The values of $y^{*}$ are well below unity, more or less in the dilute regime. This explains why they are rather close to those for 'fixed $q$ ', which are (Eq. (5.9)) 0.321 for $\mathrm{mf}$ and 0.385 for ev; for $y$ around 0.35 the non-ideal terms in Eqs. (6.17) and (6.19) are still relatively small. The $Y^{*}$ values are higher than $y^{*}$ because of the factor $\left(q_{R}^{*}\right)^{-1 / \gamma}$, with $q_{R}^{*}=0.34$ $(\mathrm{mf})$ or $0.39(\mathrm{ev})$. It is interesting to note that the 'variable $q$ ' values of $(\Pi v)^{*}$ are nearly the same as for 'fixed $q$ ', where $(\Pi v)^{*}=10.729$ (Eq. (3.7)).

The colloid concentrations in the cep are

$f^{*}=\left\{\begin{array}{l}0.466 \\ 0.463\end{array} \quad f_{s}^{*}=\left\{\begin{array}{l}1.460 \\ 1.465\end{array} \quad \eta^{*}=\left\{\begin{array}{l}0.318 \\ 0.317\end{array} \quad \eta_{s}^{*}= \begin{cases}0.593 & \mathrm{mf} \\ 0.594 & \mathrm{ev}\end{cases}\right.\right.\right.$
These values are nearly the same as for 'fixed q' (Eq. (3.7)).

We give also the value of the free volume fraction $\alpha^{*}$ in the cep:

$\alpha^{*}=\left\{\begin{array}{c}0.360 \\ 0.381\end{array} \quad \alpha_{s}^{*}=\left\{\begin{array}{cc}0.0232 & \mathrm{mf} \\ 0.0300 & \mathrm{ev}\end{array}\right.\right.$

These data may be compared with the results for 'fixed $q$ ' (Eq. (5.17)), where $\alpha^{*}=0.317$ and $\alpha_{s}^{*}=0.0120$. Because $q^{*}$ for 'variable $q^{\text {' }}\left(q^{*}=0.28\right.$ according to Eq. (6.20)) is slightly smaller than for 'fixed $q^{\prime}$ ( $\left.q^{*}=0.33\right), \alpha^{*}$ is slightly higher. For $\alpha_{s}^{*}$ we see a bigger difference, which is caused by the fact that the exclusion limit is not fully reached when $q$ decreases with increasing polymer concentration.

From $y^{*}$ the corresponding (external) polymer concentration follows as

$\varphi^{*}=y^{*} \varphi_{\mathrm{ov}}$.

where $\varphi_{\mathrm{ov}}$ depends on the radius of gyration $R$ and on the solvency $\boldsymbol{v}$ according to Eq. (6.3).

Finally, $q_{R}^{*}$ (Eq. (6.20)) determines directly the value $a^{*}$ (when $R$ is given) or the value $R^{*}$ (at given $a$ ):

$a^{*}=R / q_{R}^{*} \quad($ given $R) \quad R^{*}=a q_{R}^{*} \quad($ given $a)$.

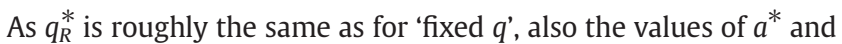
$R^{*}$ are about the same.

\subsection{Triple and critical curves}

Critical curves are computed from Eq. (2.26), with Eq. (2.23) for the derivatives of $(p v)^{p}$. The integration variable $\Pi v$ is replaced by $Y$, and the parameters $Y^{c}$ and $f^{c}$ at given $q_{R}>q_{R}^{*}$ are found from solving the two simultaneous equations of Eq. (2.26). Triple points follow from Eq. (2.28): these four equations are solved for the four parameters $Y^{t}, f_{g}^{t}$, $f_{l}^{t}$, and $f_{s}^{t}$ at given $q_{R}>q_{R}^{*}$.

\subsubsection{The parameters $Y^{c}$ and $Y^{t}$}

Fig. 6.2 gives the numerical data for $Y^{c}$ (cp, bottom) and $Y^{t}$ (tp, top) for both $\mathrm{mf}$ (closed symbols) and ev (open symbols) in a normalized representation: $\tilde{Y}=Y / Y^{*}$ as a function of $\tilde{q}_{R}=q_{R} / q_{R}^{*}$, for 17 values of $q_{R}$ as indicated in the legend, ranging from $q_{R}^{*}$ to $q_{R}=5$. The cep is thus situated at $\tilde{Y}=\tilde{q}_{R}=1$. With increasing $q_{R}$ the parameter $Y^{t}$ initially goes up, but for high $q_{R}$ a constant level $Y_{\infty}^{t}$ is reached, as expected from Eqs. (6.14) and (6.15). This final level is some 35\% higher than $Y^{*}$. In contrast, $Y^{c}$ goes down but also here a final protein-limit value $Y_{\infty}{ }^{c}$ is reached, which is about 40\% lower than $Y^{*}$. However, before reaching

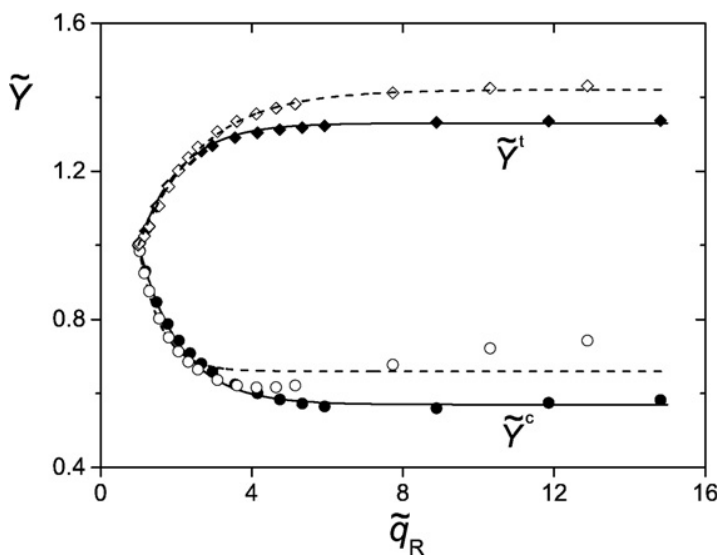

Fig. 6.2. The parameters $\tilde{Y}^{c}$ (bottom) and $\tilde{Y}^{t}$ (top) as a function of $\tilde{q}_{R}=q_{R} / q_{R}^{*}$. Symbols (closed for mf, open for ev) are the numerical data, curves (solid for mf, dashed for ev) are the analytical approximations of Eqs. (6.26), (6.27). The numerical mf data are for $q_{R}=q_{R}^{*}(=0.337), 0.35,0.4,0.5,0.6,0.7,0.8,0.9,1,1.2,1.4,1.6,1.8,2,3,4$, and 5 . Those for ev are the same except for the first three which are $q_{R}^{*}(=0.388), 0.4$, and 0.45 . 
this plateau $Y^{c}$ passes through a minimum. This minimum is weak and situated around $q_{R}=2.5\left(\tilde{q}_{R}=7.4\right)$ for $\mathrm{mf}$, and somewhat more pronounced and at smaller $q_{R}$ (around 1.6) for ev.

For interpreting the trends, it is useful to have an analytical approximation for these results. The triple curves in Fig. 6.2 are well described by an exponential curve (see Eq. (6.27)), for an accurate description of $Y^{c}\left(q_{R}\right)$ a more complicated function would be needed to capture the minimum. We keep things simple and use an exponential decay also in this case, thereby neglecting the minimum. The curves in Fig. 6.2 (solid for mf, dashed for ev) are drawn according to

$\tilde{Y}^{c}=\tilde{Y}_{\infty}^{c}+\left(1-\tilde{Y}_{\infty}^{c}\right) e^{\mu\left(1-\tilde{q}_{R}\right)}= \begin{cases}0.57+0.43 e^{0.9\left(1-\tilde{q}_{R}\right)} & \mathrm{mf} \\ 0.66+0.34 e^{1.7\left(1-\tilde{q}_{R}\right)} & \mathrm{ev}\end{cases}$

$\tilde{Y}^{t}=\tilde{Y}_{\infty}^{t}+\left(1-\tilde{Y}_{\infty}^{t}\right) e^{\mu\left(1-\tilde{q}_{R}\right)}=\left\{\begin{array}{ll}1.33-0.33 e^{0.9\left(1-\tilde{q}_{R}\right)} & \mathrm{mf} \\ 1.42-0.42 e^{0.6\left(1-\tilde{q}_{R}\right)} & \mathrm{ev}\end{array}\right.$.

In the protein limit we have then

$Y_{\infty}^{c}=\left\{\begin{array}{l}0.57 Y^{*}=0.51 \\ 0.66 Y^{*}=0.97\end{array} \quad Y_{\infty}^{t}=\left\{\begin{array}{ll}1.33 Y^{*}=1.20 & \mathrm{mf} \\ 1.42 Y^{*}=2.08 & \mathrm{ev}\end{array}\right.\right.$.

It is clear from Fig. 6.2 that the triple curves are very well described by Eq. (6.27). Eq. (6.26) gives a fair description of $Y^{c}$ for $\mathrm{mf}$, but for ev the deviations are larger: $Y^{c}$ is overestimated by $7 \%$ around the minimum $\left(q_{R}=1.6\right)$, and underestimated for high $q_{R}\left(12 \%\right.$ for $\left.q_{R}=5\right)$. For the sake of simplicity we shall accept these slight discrepancies.

\subsubsection{The parameters $(\Pi v)^{c}$ and $(\Pi v)^{t}$}

By substituting Eqs. (6.26) and (6.27) into Eq. (6.18) we find explicit analytical expressions for the quantities $(\Pi v)^{c}$ and $(\Pi v)^{t}$ as a function of $q_{R}$. In the protein limit the value $(\Pi v)_{\infty}$ is found as $(\Pi v)_{\infty}=c_{2} Y_{\infty}^{3 \gamma}$, or

$(\Pi v)_{\infty}^{c}=\left\{\begin{array}{l}0.56 \\ 1.51\end{array} \quad(\Pi v)_{\infty}^{t}=\left\{\begin{array}{ll}7.13 & \mathrm{mf} \\ 8.82 & \mathrm{ev}\end{array}\right.\right.$.

Fig. 6.3 shows the comparison of the numerical results (symbols) with the analytical equations. In addition, we compare with the 'fixed $q$ ' results of chapter 3 (dotted curves). In this case we used the ev version (Eq. (5.5), see also Table 5.1) to convert the fixed $q$ to $q_{R}$.

In the previous section we saw already that $(\Pi \mathrm{v})^{*}$ and $q_{R}^{*}$ are nearly the same for 'fix' and 'var' and for $\mathrm{mf}$ and ev: the three ceps in Fig. 6.3 are thus very close, at $(\Pi v)^{*}$ around 11 and $q_{R}^{*}$ around 0.35 . With increasing $q_{R},(\Pi v)^{t}$ drops quickly to the value $(\Pi v)_{\infty}^{t}$. In 'fix' this decay is smooth and $(\Pi v)_{\infty}^{t}=(p v)_{0}^{0}=6.08$, which corresponds to full

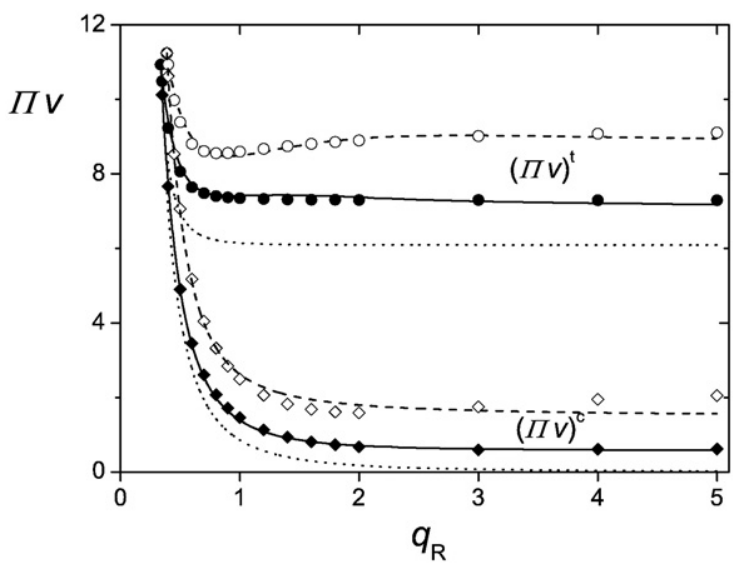

Fig. 6.3. Triple points and critical points for 'variable $q$ ' in a $\Pi v\left(q_{R}\right)$ representation. Symbols and the $q_{R}$ range are the same as in Fig. 6.2. The curves (solid for mf, dashed for ev) are analytical approximations, based on Eq. (6.18) (with $Y^{c}$ and $Y^{t}$ from Eqs. (6.26) and (6.27). The dotted curves are $\widetilde{\Pi v}^{c}$ and $\widetilde{\Pi v}^{t}$ for 'fixed $q^{\prime}$ (Eqs. (3.18) and (3.19) in combination with $\left.q \sim q_{R}{ }^{0.88}\right)$.

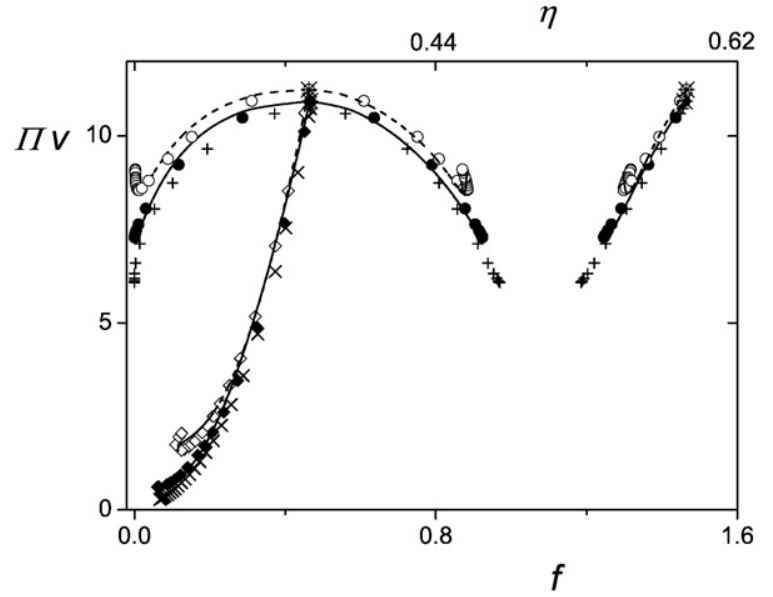

Fig. 6.4. Triple points and critical points for 'variable $q$ ' (circles and diamonds) and for 'fixed $q$ ' (crosses and plusses) in a $\Pi v(f)$ representation. Closed symbols are for $\mathrm{mf}$, open ones for ev, for the same $q_{R}$ set as in Fig. 6.2. The $q$ range for 'fixed $q$ ' is the same as in Fig. 3.3 and the corresponding $q_{R}$ 's can be read from Table 5.1; the highest $q$ is 1.2 which corresponds to $q_{R}=1.32$ (mf) or $1.44(\mathrm{ev})$. The curves (solid for $\mathrm{mf}$, dashed for ev) are analytical approximations, based on Eq. (6.18) (with $Y^{c}$ and $Y^{t}$ from Eqs. (6.26) and (6.27)) for $\Pi v\left(q_{R}\right)$, and Eqs. (6.35)-(6.38) for $\eta^{c}\left(q_{R}\right), \eta_{g}^{t}\left(q_{R}\right), \eta_{l}^{t}\left(q_{R}\right)$, and $\eta_{s}^{t}\left(q_{R}\right)$, whereby the $\eta$ 's were converted to $f$ using $f=\eta /(1-\eta)$.

exclusion of polymer from the condensed phases. In 'var' there is a weak intermediate minimum, which is nicely captured in the analytical model. Moreover, since now the depletion layers are compressed for high $q_{R}$ (more strongly in ev than in $\mathrm{mf}$ ), the final level $(\Pi v)_{\infty}^{t}$ is higher than for 'fix' (and higher in ev than in mf: 8.82 and 7.13 , respectively). Nevertheless, the quantitative differences in $(\Pi v)^{t}\left(q_{R}\right)$ between 'fix' and 'var' are small, which illustrates the central role of the parameter $\Pi v$ in the thermodynamics of colloid/polymer mixtures. This nearly universal behavior does not hold for the parameter $y^{t}$, which is greatly different in the two cases; we return to this point in Section 6.4.4.

The parameter $(\Pi v)^{c}$ decays to zero for 'fixed $q^{\prime}$ : $(\Pi v)^{c} \sim q^{-2.6} \sim q_{R}^{-2.3}$ according to Eqs. (3.18) and (5.5). For 'var' the initial decay is about the same but there is a final level $(\Pi v)_{\infty}{ }^{c}=0.56(\mathrm{mf})$ or $1.51(\mathrm{ev})$. Again the analytical model describes the numerical data in Fig. 6.3 quite well, although we do not capture the weak minimum for ev; this is because Eq. (6.26) neglects the minimum of $Y^{c}$ in Fig. 6.2.

It is illustrative to also show a $\Pi v(f)$ diagram, just like Fig. 3.3 for 'fix'. Fig. 6.4 gives three sets of such critical and triple curves. The circles are triple points for 'variable $q$ ' and the diamonds represent the corresponding critical points; closed symbols are for mf, open ones for ev. The curves (solid for $\mathrm{mf}$, dashed for ev) are analytical approximations based upon $(\Pi v)\left(q_{R}\right)$ (as in Fig. 6.3) and $\eta\left(q_{R}\right)$ (given in Section 6.4.5). The crosses (cp) and plusses (tp) are the 'fix' results and are the same as in Fig. 3.3; in this case we did not repeat the analytical curves from Fig. 3.3.

Again we see a nearly universal behavior for $(\Pi v)^{c}$ and $(\Pi v)^{t}$ in the three cases. The minor quantitative differences are due to a slightly different cep and different final levels for $Y^{c}$ and $Y^{t}$ and, hence, for $(\Pi v)^{c}$ and $(\Pi v)^{t}$, which we discussed in connection with Fig. 6.3. We note that also in Fig. 6.4 the analytical equations describe the numerical data quite well.

\subsubsection{The parameters $q^{c}$ and $q^{t}$}

Inserting Eqs. (6.26) and (6.27) into Eq. (6.17) gives explicit analytical approximations for $q^{c}$ and $q^{t}$ as a function of $q_{R}$. Fig. 6.5 compares these approximations (solid curves for mf, dashed for ev) with the numerical results (closed symbols for mf, open for ev). In this case we use again a normalized representation $\tilde{q}$ as a function of $\tilde{q}_{R}$ so that the cep is the point $(1,1)$. The dotted curve in this figure is the 


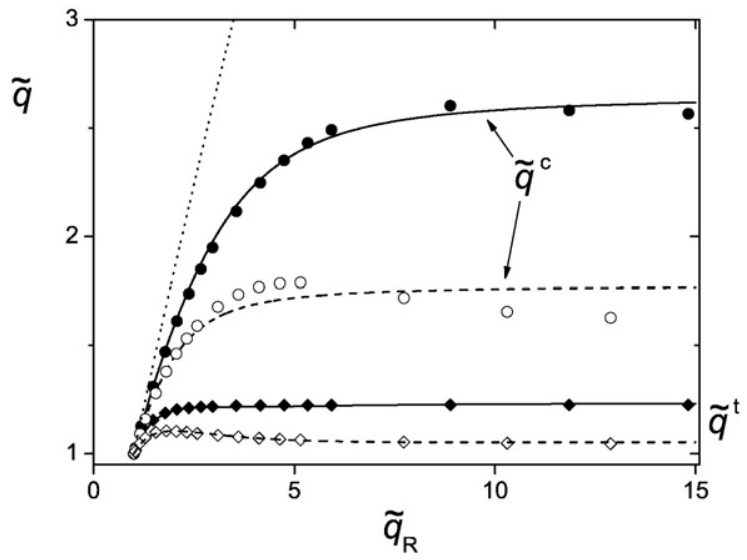

Fig. 6.5. The parameters $\tilde{q}^{c}$ (top) and $\tilde{q}^{t}$ (bottom) as a function of $\tilde{q}_{R}=q_{R} / q_{R}^{*}$. Symbols (closed for mf, open for ev) are the numerical data, curves (solid for mf, dashed for ev) are Eq. (6.17) with the analytical approximations of Eqs. (6.26) and (6.27) for $Y^{c}$ and $Y^{t}$ The numerical data are for the same $q_{R}$ set as in Fig. 6.2. The dotted curve is the 'fix' result $\tilde{q}=\tilde{q}_{R}{ }^{0.88}$.

'fixed $q$ ' result $\tilde{q}=\tilde{q}_{R}{ }^{0.88}$ according to Eq. (5.5) (this is the ev version but the $\mathrm{mf}$ result $\tilde{q}=\tilde{q}_{R}{ }^{0.9}$ is hardly different).

For 'fix' $q$ is a simple power law: $q$ is of order $q_{R}$ and increases without bounds. This is because the depletion thickness is assumed to be independent of the polymer concentration; only curvature effects are taken into account. For 'var' the depletion layer is compressed at high polymer concentration (high $q_{R}$ ), and in semidilute solutions $\delta$ becomes independent of $R$, so $q$ becomes independent of $q_{R}$.

According to Eq. (6.17) with $Y_{\infty}$ from Eq. (6.28) the final value $q_{\infty}$ is

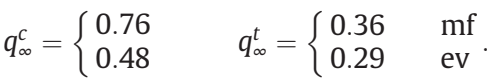

For $q^{t}$ the variation with $q_{R}$ in ev is quite small, with $q_{\infty}^{t}$ only a few per cent higher than $q^{*}$. The weak maximum in $q^{t}\left(q_{R}\right)$ in this case is nicely reproduced in the analytical model. For mf there is a smooth transition from $q^{*}$ to $q_{\infty}{ }^{t}$ and the ratio $q_{\infty}^{t} / q^{*}$ is somewhat higher, but even here the total variation is only around $20 \%$.

The increase in $q^{c}$ is stronger: $q_{\infty}^{c} / q^{*}$ is around 2.6 for $\mathrm{mf}$ and around 1.8 for ev. In the latter case we see again a non-monotonic behavior in the numerical $q^{c}\left(q_{R}\right)$, which is disregarded in the analytical model. The minimum in $Y^{c}\left(q_{R}\right)$ translates itself into a maximum in $q^{c}\left(q_{R}\right)$ because $q$ decreases with increasing $Y$.

We see from Eq. (6.30) and Fig. 6.5 that $q$ in tp and $c p$ (hence, along the GL binodals) remains small throughout: its value well below unity in all cases. This is important since our model is based upon scaledparticle theory (Eqs. (2.5)-(2.7)), which agrees with simulations $[10,11,28,29]$ for $q$ up to order unity, but breaks down for higher $q$ values. Unlike in 'fix', where $q$ diverges with $q_{R}$, our 'variable $q$ ' model remains within the validity range of scaled-particle theory.

\subsubsection{The parameters $y^{c}$ and $y^{t}$}

Using Eqs. (6.26) and (6.27) with $y=Y q_{R}^{1 / \gamma}$ we find immediately the analytical equations for $y^{c}$ and $y^{t}$ :

$\tilde{y}^{c}=\tilde{q}_{R}^{1 / \gamma}\left[\tilde{Y}_{\infty}^{c}+\left(1-\tilde{Y}_{\infty}^{c}\right) e^{\mu\left(1-\tilde{q}_{R}\right)}\right]=\left\{\begin{array}{ll}\tilde{q}_{R}\left[0.57+0.43 e^{0.9\left(1-\tilde{q}_{R}\right)}\right] & \mathrm{mf} \\ \tilde{q}_{R}^{1.3}\left[0.66+0.34 e^{1.7\left(1-\tilde{q}_{R}\right)}\right] & \mathrm{ev}\end{array}(6.31)\right.$

$\tilde{y}^{t}=\tilde{q}_{R}^{1 / \gamma}\left[\tilde{Y}_{\infty}^{t}+\left(1-\tilde{Y}_{\infty}^{t}\right) e^{\mu\left(1-\tilde{q}_{R}\right)}\right]= \begin{cases}\tilde{q}_{R}\left[1.33-0.33 e^{0.9\left(1-\tilde{q}_{R}\right)}\right] & \mathrm{mf} \\ \tilde{q}_{R}^{1.3}\left[1.42-0.42 e^{0.6\left(1-\tilde{q}_{R}\right)}\right] & \mathrm{ev}\end{cases}$

Fig. 6.6 shows $\tilde{y}^{c}=y^{c} / y^{*}$ (diamonds) and $\tilde{y}^{t}=y^{t} / y^{*}$ (circles) as a function of $\tilde{q}_{R}=q_{R} / q_{R}^{*}$ on a double-logarithmic scale. Symbols (closed for $\mathrm{mf}$, open for ev) are numerical data, curves (solid for mf, dashed for ev) are Eqs. (6.31) and (6.32). The agreement is excellent. For comparison we give in Fig. 6.6 also $\tilde{y}^{c}$ and $\tilde{y}^{t}$ for 'fix' (dotted); these curves are taken from Fig. 5.2 (Eqs. (5.10) and (5.11)).

In Fig. 5.2 we found that $y^{c}\left(q_{R}\right)$ for 'fix' is a simple power law $\tilde{y}^{c}=\tilde{q}_{R}^{0.72}$ : this is the straight line with label 'cp fix' in Fig. 6.6. On the other hand, $y^{t}$ for 'fix' is a double power law $y^{t} \sim q_{R}^{1.4}$ for $q_{R}$ close to $q_{R}^{*}$ and $y^{t} \sim q_{R}^{3}$ for high $q_{R}$.

Despite the different functional dependencies in Eq. (5.11) for 'fix' (where $y / y_{\infty}-1$ is a power law in $q_{R}$ ) and Eqs. (6.30) and (6.31) for 'var' (where $y / y_{\infty}-1$ is exponential in $q_{R}$ ), in both cases the limits are power laws. For $y^{t}$ in ev these two limits are indicated in Fig. 6.6 as the dashdot lines. The protein limit is obvious: $y_{\infty}=Y_{\infty} q_{R}^{1 / \gamma}$ or, with Eq. (6.28):

$y_{\infty}^{c}=\left\{\begin{array}{l}0.51 q_{R} \\ 0.97 q_{R}^{1.3}\end{array} \quad y_{\infty}^{t}=\left\{\begin{array}{ll}1.20 q_{R} & \mathrm{mf} \\ 2.08 q_{R}^{1.3} & \mathrm{ev}\end{array} \quad\right.\right.$ (protein limit).

For $q_{R}$ quite close to $q_{R}^{*}$ we use Eqs. (6.31) and (6.32) in the form $\left.z=x^{\kappa}\left[A+(1-A) e^{\mu(1-x}\right)\right]$. From evaluating $d \ln z / d x=d z / d x$ at $x=z=1$ it follows that the exponent $\lambda$ in $z=x^{\lambda}$ for $z$ and $x$ close to unity is given by $\lambda=\kappa+\mu(A-1)$, the same result as found from Eq. (5.2). Hence, $\lambda=1 /$ $\gamma-\mu\left(Y_{\infty}-1\right)$. We find

$y^{c}=\left\{\begin{array}{l}0.59 q_{R}^{0.61} \\ 0.85 q_{R}^{0.72}\end{array} \quad y^{t}=\left\{\begin{array}{ll}1.24 q_{R}^{1.30} & \text { mf } \\ 1.86 q_{R}^{1.55} & \text { ev }\end{array} \quad\right.\right.$ (colloid limit). (6.34)

We see that $\lambda<1 / \gamma$ for $y^{c}$ : the initial slope is smaller than the final one and the curves bend upwards in the transition region. For $y^{t}$ we have $\lambda>1 / \gamma$ and a (weak) downward trend is found.

\subsubsection{The parameters $\eta^{c}$ and $\eta^{t}$}

In chapter 3 we concluded that for 'fixed $q^{\prime} f^{c}$ for the critical point could be described as a power law $f^{c} \sim q^{-2.6}$ (Eq. (3.18)). For the solid branch of the triple point we found in Eq. (3.20) a simple power-law decay of $\tilde{f}_{s}^{t}$ from $\tilde{f}_{s}^{t}=1$ in the cep to a final value $\tilde{f}_{s}^{e}=f_{s}^{0} / f_{s}^{*}=0.814$; this decay could be written as $\tilde{f}_{s}^{t}=\tilde{f}_{s}^{e}+\left(1-\tilde{f}_{s}^{e}\right) \tilde{q}^{-4.6}$. For the gas and liquid branches such a power law did not work because $f(q)$ around the cep behaves more or less parabolic (Fig. 3.6); here a 'parabolic power law' of the type $\tilde{f}=1+\left(\tilde{f}^{e}-1\right) \sqrt{1-\tilde{q}^{-4.6}}$ (which is a simplified version of Eqs. (3.22) and (3.24)) works better. In the above expressions $\tilde{q}$ may be replaced by (roughly) $\tilde{q}_{R}^{0.9}$ on the basis of Eq. (5.5).

We try similar dependencies for the colloid concentrations in the case of 'variable $q$ '. We expect that we have to adjust the exponents, and we change from the parameter $f$ to the parameter $\eta=f /(1+f)$, which is directly measured in an experiment. Moreover, we do not

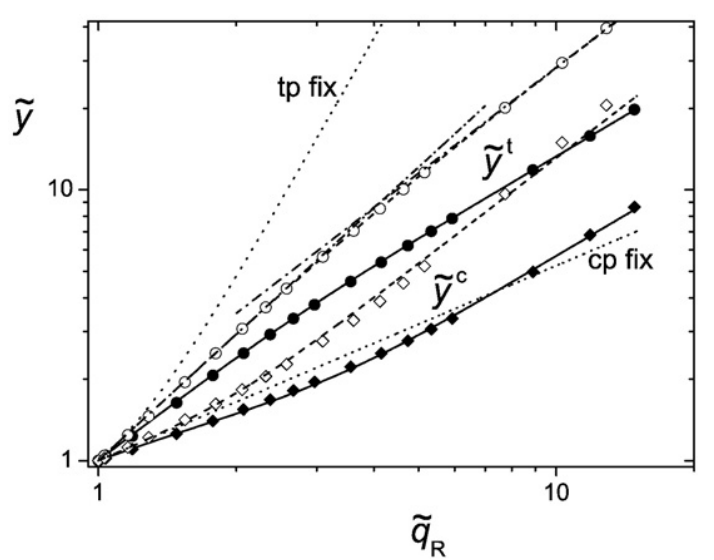

Fig. 6.6. The parameters $\tilde{y}^{t}$ (circles) and $\tilde{y}^{c}$ (diamonds) as a function of $\tilde{q}_{R}$, on a doublelogarithmic scale. Closed symbols are for $\mathrm{mf}$, open ones for ev. The curves (solid for $\mathrm{mf}$, dashed for ev) are the analytical approximations of Eqs. (6.31) and (6.32). For the top curve $\left(\tilde{y}^{t}\right.$ for ev) the limiting initial and final asymptotes $\left(\tilde{y}^{t}=\tilde{q}_{R}^{\lambda}\right.$ and $\tilde{y}^{t}=\mathrm{A} \tilde{q}_{R}^{\kappa}$, respectively) are indicated as the dash-dotted lines. The dotted curves are $\tilde{y}^{t}$ and $\tilde{y}^{c}$ for 'fixed $q$ ' according to Eqs. (5.10) and (5.11). 
consider the parameter $q$ but the parameter $q_{R}$, again directly accessible in experiment.

For the dependence $\eta^{c}\left(q_{R}\right)$ a power law $\eta^{c} \sim q_{R}^{-x}$ works satisfactorily for $q_{R}$ below (roughly) 2, but for high $q_{R}$ a final level for $\eta^{c}$ is reached. A fair approximation for $\eta^{c}$ turns out to be

$\tilde{\eta}^{c}=\left\{\begin{array}{l}\max \left(\tilde{q}_{R}^{-0.74}, 0.185\right) \\ \max \left(\tilde{q}_{R}^{-0.63}, 0.33\right)\end{array} \quad \eta_{\infty}^{c}=\left\{\begin{array}{l}0.059 \mathrm{mf} \\ 0.104 \mathrm{ev}\end{array}\right.\right.$

which implies a discontinuous behavior: a power law $\eta^{c} \sim q_{R}^{-\mathrm{x}}(\mathrm{x}=0.74$ or 0.63 ) for small $q_{R}$ (colloid limit) and a constant level $\left(\eta^{c}=0.185 \eta^{*}=\right.$ 0.059 or $0.33 \eta^{*}=0.104$ ) for high $q_{R}$ (protein limit). Fig. 6.7 shows the comparison between the numerical values (diamonds) and Eq. (6.35), in a representation $\tilde{\eta}^{c}$ as a function of $1 / \tilde{q}_{R}$, which is the same as in Fig. 3.6. We note that the exponents in Eq. (6.35) are considerably

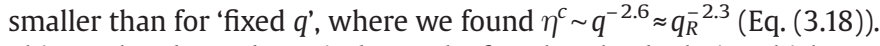
This weaker dependence is due to the fact that the depletion thickness decreases with increasing polymer concentration.

For $\eta_{g}^{t}$ and $\eta_{l}^{t}$ we try a 'parabolic power law' $\tilde{\eta}^{t}=1+\left(\tilde{\eta}_{\infty}^{t}-1\right) \sqrt{1-\tilde{q}_{R}^{-x}}$. In the gas branch $\eta_{g, \infty}^{t}$ is zero, and $x=3.5$ for $\mathrm{mf}$ and $x=3$ for ev gives a fair description of the numerical data:

$\tilde{\eta}_{g}^{t}=\left\{\begin{array}{ll}1-\sqrt{1-\tilde{q}_{R}^{-3.5}} & \mathrm{mf} \\ 1-\sqrt{1-\tilde{q}_{R}^{-3}} & \text { ev }\end{array} \quad \eta_{g, \infty}^{t}=0\right.$.

The lower branch in Fig. 6.7 demonstrates that Eq. (6.36) is not perfect, but the trends are described rather well. The exponents in Eq. (6.36) are slightly smaller than for 'fixed $q$ ', where we used $\tilde{q}^{-4.6} \approx$ $\tilde{q}_{R}^{-4.1}$ (Eq. (3.24)).

In the liquid branch we have a non-zero final value $\eta_{l, \infty}^{t}$. For 'fixed $q^{\prime}$, where for high $q$ the full exclusion limit is reached, $\eta_{l, \infty}^{t}=\eta_{f}^{0}=0.492$ or $\tilde{\eta}_{l, \infty}^{t}=\eta_{f}^{0} / \eta^{*}=1.545$. For 'variable $q$ ' this limit is not fully attained and $\eta_{l, \infty}^{t}$ is somewhat smaller. The liquid branch of the triple curve (top curves in Fig. 6.7) is reasonably described by

$\tilde{\eta}_{l}^{t}=\left\{\begin{array}{l}1+0.51 \sqrt{1-\tilde{q}_{R}^{-4}} \\ 1+0.48 \sqrt{1-\tilde{q}_{R}^{-4}}\end{array} \quad \eta_{l, \infty}^{t}=\left\{\begin{array}{ll}0.480 & \mathrm{mf} \\ 0.469 & \mathrm{ev}\end{array}\right.\right.$.

In this case the exponent is quite close to the 'fixed $q$ ' value in Eq. (3.22) $\left(\tilde{q}^{-4.6} \approx \tilde{q}_{R}^{-4.1}\right)$.

Finally, we use a simple power law $\tilde{\eta}=1+\left(\tilde{\eta}_{\mathrm{s}, \infty}^{t}-1\right) \tilde{q}_{R}^{-x}$ for the solid branch. For 'fixed $q$ ' the final value is $\eta_{\mathrm{s}, \infty}^{t}=\eta_{\mathrm{s}}^{0}=0.542$, corresponding to

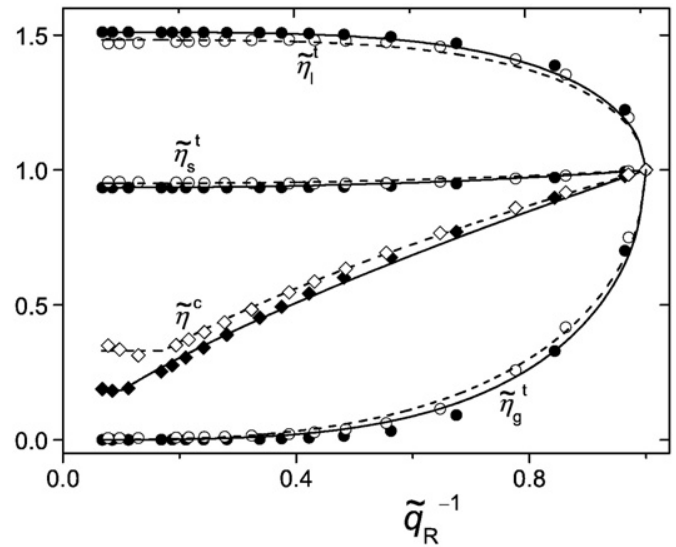

Fig. 6.7. Numerical data for $\tilde{\eta}^{c}$ (diamonds) and for $\tilde{\eta}_{g}^{t}$, $\tilde{\eta}_{l}^{t}$, and $\tilde{\eta}_{s}^{t}$ (circles) as a function of $1 / \tilde{q}_{R}$. Closed symbols are for $\mathrm{mf}$, open ones for ev. The curves (solid for $\mathrm{mf}$, dashed for ev) are the analytical approximations of Eqs. (6.35)-(6.38).

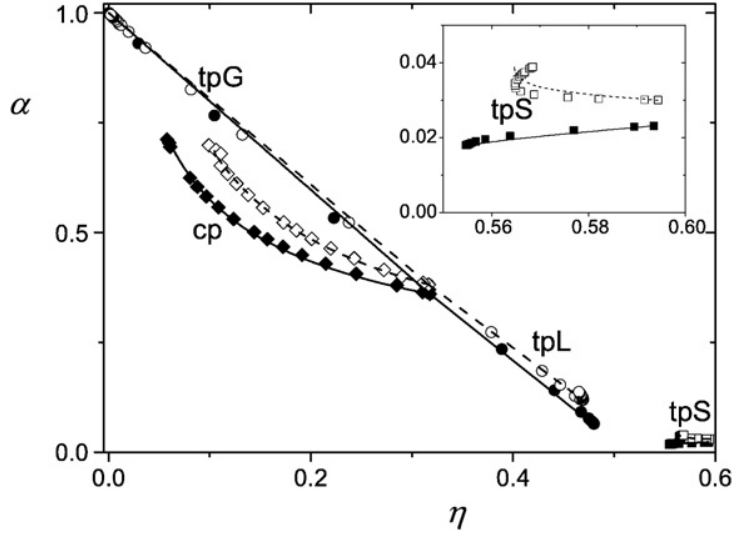

Fig. 6.8. The free volume fraction $\alpha^{t}$ for triple points (three branches tpG, tpL, tpS, circles) and $\alpha^{c}$ for critical points (cp, diamonds) as a function of the corresponding colloid volume fractions $\eta^{t}$ and $\eta^{c}$. Symbols (closed for mf, open for ev) are the numerical data for the standard set of $q_{R}$ 's (see Fig. 6.2). Curves are analytical according to Eqs. (6.41) and (6.43) for tpG and tpL, Eq. (6.45) in combination with Eq. (6.38) for tpS, and Eq. (6.39) for cp. The inset shows more detail of the solid part of the triple point.

$\tilde{\eta}_{s, \infty}^{t}=\eta_{s}^{0} / \eta_{s}^{*}=0.914$. For 'variable $q$ ' the exclusion limit is not fully reached:

$\tilde{\eta}_{s}^{t}=\left\{\begin{array}{cc}0.935+0.065 \tilde{q}_{R}^{-3} & \mathrm{mf} \\ 0.95+0.05 \tilde{q}_{R}^{-3} & \mathrm{ev}\end{array} \quad \eta_{s, \infty}^{t}=\left\{\begin{array}{cc}0.555 \mathrm{mf} \\ 0.565 \mathrm{ev}\end{array}\right.\right.$.

On the scale of Fig. 6.7 Eq. (6.38) seems to work excellently, but upon close inspection it is again not perfect: it does not capture the weak minimum in $\eta_{s}^{t}\left(q_{R}\right)$ for ev (which is hardly visible in Fig. 6.7). Nevertheless, the overall trends are reasonably described by the simple Eqs. (6.35)-(6.38).

\subsubsection{The parameters $\alpha^{c}$ and $\alpha^{t}$}

The free volume fraction $\alpha(\eta, q)$ is obtained from Eq. (2.5), inserting the concentration-dependent $q$ given by Eq. (6.17). Numerical values for $\alpha^{t}$ in the triple point and $\alpha^{c}$ in the critical point are given as the symbols (circles for tp, diamonds for $\mathrm{cp}$ ) in Figs. (6.8) and (6.9). In Fig. 6.8, which is the analogue of Fig. 5.10 for 'fixed $q$ ', $\alpha^{c}$ and the three $\alpha^{t}$ 's are plotted as a function of $\eta^{c}$ and the three $\eta^{t}$ 's, respectively. Fig. 6.9, which may be compared with Fig. 5.11 for 'fixed $q$ ', gives $\tilde{\alpha}^{c}$ and $\tilde{\alpha}^{t}$ as a function of $1 / \tilde{\mathrm{q}}_{R}=q_{R}^{*} / q_{R}$. The curves in Figs. 6.8 and 6.9 (solid for $\mathrm{mf}$, dashed for ev) are analytical approximations as discussed below.

We first consider $\alpha^{c}$ in the critical point. In Fig. 5.10 we saw that for 'fixed $q^{\prime} \alpha^{c}$ increases very weakly with decreasing $\eta^{c}$ (Eq. (5.21)). In Fig. 6.8 the increase is much stronger. The reason is that for 'variable $q$ ' the ratio $q / q_{R}$ decreases with increasing polymer concentration, which increases $\alpha$. The dependence of $\alpha^{c}$ on $q_{R}$ is likewise stronger in Fig. 6.9 than in Fig. 5.11.

The dependence $\alpha^{c}\left(\eta^{c}\right)$ in Fig. 6.8 is well described by a simple power law:

$\alpha^{c}=\alpha^{*}\left(\tilde{\eta}^{c}\right)^{-x}= \begin{cases}0.360\left(\tilde{\eta}^{c}\right)^{-0.41} & \mathrm{mf} \\ 0.381\left(\tilde{\eta}^{c}\right)^{-0.52} & \mathrm{ev}\end{cases}$

In Eq. (6.35) we found a power law $\tilde{\eta}^{c}=\tilde{q}_{R}^{-0.74}(\mathrm{mf})$ or $\tilde{\eta}^{c}=\tilde{q}_{R}^{-0.63}$ (ev) which applies down to a final value $\eta_{\infty}^{c}$ ( 0.185 for $\mathrm{mf}, 0.33 \mathrm{for} \mathrm{ev}$ ), after which $\tilde{\eta}^{c}$ remains constant. Also $\alpha^{c}\left(q_{R}\right)$ may be described by such a combination of a power law and a final level:

$\tilde{\alpha}^{c}=\left\{\begin{array}{l}\min \left(\tilde{q}_{R}^{0.30}, 1.96\right) \\ \min \left(\tilde{q}_{R}^{0.33}, 1.80\right)\end{array} \quad \alpha_{\infty}^{c}=\left\{\begin{array}{cc}0.706 & \mathrm{mf} \\ 0.686 & \mathrm{ev}\end{array}\right.\right.$.

The exponents in Eq. (6.40) are a combination of those in Eqs. (6.39) and (6.35): $0.41 \cdot 0.74=0.30,0.52 \cdot 0.63=0.33$. The final 


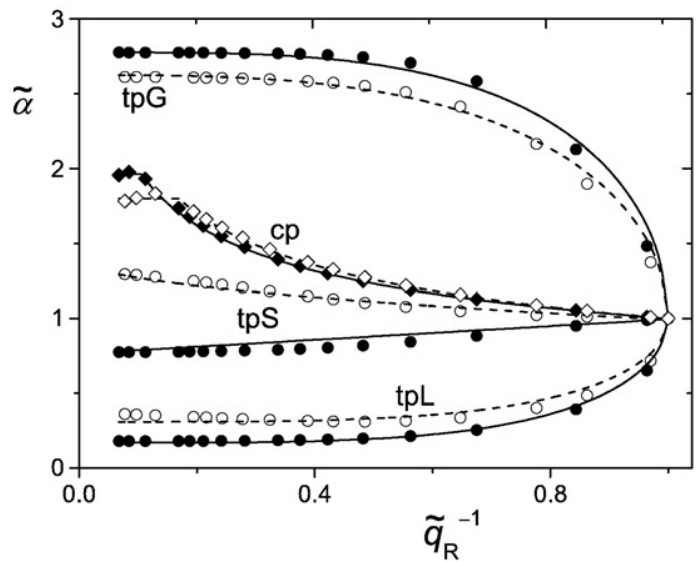

Fig. 6.9. Variation of $\tilde{\alpha}^{t}$ for triple points (three branches tpG, tpL, tpS) and of $\alpha^{c}$ for critical points (cp) with $1 / \tilde{q}_{R} \equiv q_{R}^{*} / q_{R}$. Symbols (circles for tp, diamonds for $\mathrm{cp}$, solid for $\mathrm{mf}$, open for ev) are the numerical data for the standard set of $q_{R}$ 's (see Fig. 6.2). Solid (mf) and dashed (ev) curves are analytical according to Eqs. (6.40), (6.42), (6.44), and (6.45).

high- $q_{R}$ levels of $\alpha^{c}$ are $1.96 \alpha^{*}=0.706$ for $\mathrm{mf}$ and $1.80 \alpha^{*}=0.686$ for ev. Fig. 6.9 shows that Eq. (6.40) describes the numerical data for $\alpha^{c}\left(q_{R}\right)$ quite nicely.

For the gas branch of the triple curve we see in Fig. 6.8 an approximate linear dependence of $\alpha_{g}^{t}$ on $\eta_{g}^{t}$. (In Fig. 5.10 the dependence is qualitatively the same, but it is not quite linear, see Eq. (5.18)). The approximate linearity in Fig. 6.8 can be understood from Eq. (2.2): $\alpha_{g}^{t}=1-\eta_{g}^{t}\left(1+q^{t}\right)^{3}$, which gives a straight line when $q^{t}$ is small and constant (i.e., independent of $q_{R}$ ). Both conditions are approximately met, see Fig. 6.5. We interpret the 'variable q' data for the gas branch of the triple curve with

$\alpha_{g}^{t}=1-\left(1-\alpha^{*}\right) \tilde{\eta}_{g}^{t}=\left\{\begin{array}{ll}1-2.01 \eta_{g}^{t} & \mathrm{mf} \\ 1-1.95 \eta_{g}^{t} & \mathrm{ev}\end{array}\right.$.

Fig. 6.8 shows that Eq. (6.41) overestimates $\alpha_{\mathrm{g}}^{t}$ in the intermediate range for $\alpha_{g}^{t}$ slightly. We could improve on this by writing $\alpha_{g}^{t}=1-(1-$ $\left.\alpha^{*}\right)\left(\eta_{g}\right)^{x}$ with an exponent $x$ which is just below unity, but for the sake of simplicity we will not do that.

By substituting $\tilde{\eta}_{g}^{t}\left(q_{R}\right)$ from Eq. (6.36) into Eq. (6.41) we find the explicit dependence of $\alpha_{\mathrm{g}}^{t}$ on $q_{R}$ :

$\alpha_{g}^{t}=\alpha^{*}+\left(1-\alpha^{*}\right) \sqrt{1-\tilde{q}_{R}^{-x}}=\left\{\begin{array}{ccc}0.36+0.64 \sqrt{1-\tilde{q}_{R}^{-3.5}} & \mathrm{mf} \\ 0.38+0.62 \sqrt{1-\tilde{q}_{R}^{-3}} & \text { ev }\end{array} \quad \alpha_{g, \infty}^{t}=1\right.$.

As shown by the top curves in Fig. 6.9, the simple Eq. (6.42) gives a reasonable approximation.

The liquid branch $\alpha_{l}^{t}(\eta)$ of the triple curve in Fig. 6.8 is a straight line, as was the case for 'fixed $q$ ' in Fig. 5.10. The difference between the two cases is the end value $\alpha_{l, \infty}^{t}$ and $\eta_{l, \infty}^{t}$. For 'fixed $q$ ' a reasonable approximation was $\alpha_{l, \infty}^{t} \approx 0$ and $\eta_{l, \infty}^{t} \approx \eta_{f}^{0}=0.492$. When the decrease of the depletion thickness with increasing polymer concentration is accounted for, as we do here, the full exclusion limit is not reached and $\eta_{l, \infty}^{t}$ is smaller (Eq. (6.37)): $\eta_{l, \infty}^{t}=0.480(\mathrm{mf})$ or $0.469(\mathrm{ev})$. The parameter $\alpha_{l, \infty}^{t}$ has a non-zero value, as can be seen in Figs. 6.8 and 6.9: $\alpha_{l, \infty}^{t}=$ $0.17 \alpha^{*}=0.061(\mathrm{mf})$ and $\alpha_{l, \infty}^{t}=0.31 \alpha^{*}=0.118(\mathrm{ev})$. Hence, the straight line for the liquid branch of the triple curve in Fig. 6.8 is described by

$\alpha_{l}^{t}=\alpha_{l, \infty}^{t}+\frac{\eta_{l, \infty}^{t}-\eta_{l}^{t}}{\eta_{l, \infty}^{t}-\eta^{*}}\left(\alpha^{*}-\alpha_{l, \infty}^{t}\right)=\left\{\begin{array}{ll}0.947-1.846 \eta_{g}^{t} & \mathrm{mf} \\ 0.929-1.730 \eta_{g}^{t} & \mathrm{ev}\end{array}\right.$.
Upon substituting Eq. (6.37) we obtain

$$
\begin{aligned}
\alpha_{l}^{t} & =\alpha^{*}+\left(\alpha_{l, \infty}^{t}-\alpha^{*}\right) \sqrt{1-\tilde{q}_{R}^{-4}} \\
& =\left\{\begin{array}{l}
0.360-0.299 \sqrt{1-\tilde{q}_{R}^{-4}} \\
0.381-0.263 \sqrt{1-\tilde{q}_{R}^{-4}}
\end{array} \quad \alpha_{l, \infty}^{t}=\left\{\begin{array}{cc}
0.061 & \mathrm{mf} \\
0.118 & \mathrm{ev}
\end{array}\right.\right.
\end{aligned}
$$

In Fig. 6.9 (lower curves) we see that Eq. (6.44) describes the numerical data satisfactorily.

For the solid branch of the triple curve $\alpha_{s}^{t}$ is small but non-zero. From the inset in Fig. 6.8 it is seen that, starting from the cep, $\alpha_{\mathrm{s}}^{t}$ varies only slightly, with a weak decrease of $\alpha_{s}^{t}$ with decreasing $\eta_{\mathrm{s}}^{t}$ for mf, and a weak increase for ev. The same opposing trends occur for $\alpha_{s}^{t}$ as a function of $q_{R}$ (Fig. 6.9). Despite this different behavior, it turns out that $\alpha_{s}^{t}\left(q_{R}\right)$ can be described reasonably well by the same power-law form as in Eq. (6.38) for $\eta_{s}^{t}\left(q_{R}\right)$ :

$$
\begin{aligned}
& \alpha_{s}^{t}=\alpha_{s, \infty}^{t}+\left(\alpha_{s}^{*}-\alpha_{s, \infty}^{t}\right) \tilde{q}_{R}^{-x} \\
& =\left\{\begin{array}{l}
0.0178\left(1+0.3 \tilde{q}_{R}^{-1}\right) \\
0.042\left(1-0.29 \tilde{q}_{R}^{-0.5}\right)
\end{array} \quad \alpha_{s, \infty}^{t}=\left\{\begin{array}{ll}
0.0178 & \mathrm{mf} \\
0.042 & \mathrm{ev}
\end{array} .\right.\right.
\end{aligned}
$$

The opposing trends are captured by choosing $\alpha_{\mathrm{s}, \infty}^{t}(0.0178$ for $\mathrm{mf}$, 0.042 for ev) below or above $\alpha_{\mathrm{s}}^{*}$. Fig. 6.9 demonstrates that Eq. (6.45) for $\alpha_{s}^{t}\left(q_{R}\right)$ works reasonably well.

\subsection{Phase diagrams in terms of the reduced polymer concentration $y$}

\subsubsection{FS binodals}

In Fig. 5.3 we displayed FS binodals for a good solvent in terms of $y(f)$ for the case of 'fixed q'. Fig. 6.10 gives the analogous results for 'variable $q$ '. The overall picture is the same, but there are important quantitative differences. These are only minor around and below the cep, where the dilute limit applies. As $q_{R}$ increases these quantitative differences become more pronounced, which can be read from $y=y^{t}$ at the triple point. We saw in Section 6.4.4 that $y^{t}$ increases more slowly with $q_{R}$ for 'variable $q$ '. For example, in Fig. 6.10 for $q_{R}=0.8 y^{t}$ is around 1.3 , in the crossover region between the dilute and

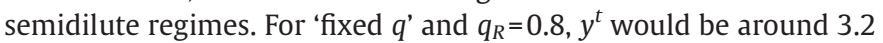
Eq. (5.11), well into the semidilute region and outside the range shown in Fig. 6.10. Hence, the differences between Figs. 6.10 and 5.3 are caused mainly by the dependence $y^{t}\left(q_{R}\right)$, which for high $q_{R}$ scales as $y^{t} \sim q_{R}^{3}$ in Fig. 5.3, and as $y^{t} \sim q_{R}^{1.3}$ in Fig. 6.10.

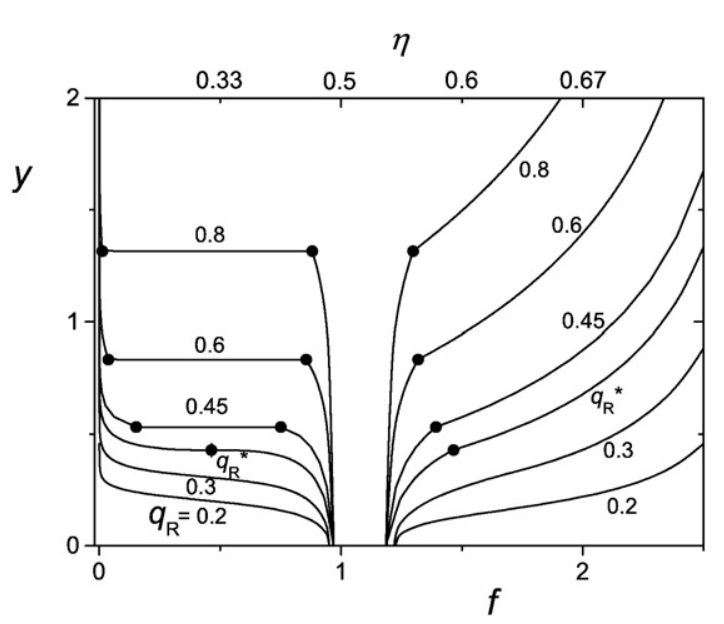

Fig. 6.10. FS binodals $y(f)$ for 'variable $q$ ' and a good solvent. The binodals are, from bottom to top, for $q_{R}=0.2,0.3,0.388\left(=q_{R}^{*}\right), 0.45,0.6$, and 0.8 . The circles indicate the triple points for the four highest $q_{R}$ values; the triple point for $q_{R}=q_{R}^{*}$ is the cep. 


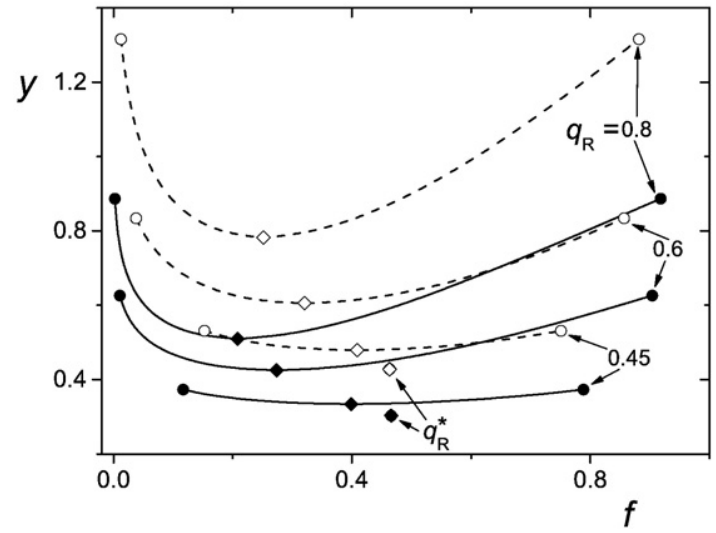

Fig. 6.11. GL binodals $y(f)$ for 'variable $q$ ', for both $\mathrm{mf}$ (solid curves, closed symbols) and ev (dashed, open). The binodals are, from bottom to top, for $q_{R}=q_{R}^{*}$ (point binodal), 0.45 , 0.6 , and 0.8 . The circles are the (fluid parts of the) triple points, diamonds are critical points.

\subsubsection{GL binodals}

Fig. 6.11 gives 'variable $q$ ' GL binodals $y(f)$ for both $\mathrm{mf}$ (solid curves, closed symbols) and ev (dashed, open), for $q_{R}=q_{R}^{*}$ (point binodal), $0.45,0.6$, and 0.8 . This figure may be compared with Fig. 5.4 for 'fixed $q^{\prime}$. Again the qualitative picture is the same in both figures, and again there are quantitative differences. These are caused by the different dependences of both $y^{t}$ (tp) and $y^{c}(\mathrm{cp})$ on $q_{R}$. For 'variable $q^{\prime}, y^{c} \sim q_{R}$ (mf) or $y^{c} \sim q_{R}^{1.3}$ (ev) for high $q_{R}$ (which is the same scaling as for $y^{t}$ ), for 'fixed $q^{\prime}$ the scaling is $y^{c} \sim q_{R}^{0.72}$ (Eq. (5.10)). Hence, whereas $y^{t}$ depends more weakly on $q_{R}$ in Fig. 6.11 as compared to Fig. 5.4, $y^{c}$ varies more strongly with $q_{R}$.

We may also consider the ratio $y^{t} / y^{c}$, which is a measure for the 'liquid window', the region $y^{c}<y<y^{t}$. For 'fixed $q$ ' this ratio goes as $q_{R}^{3} /$ $q_{R}^{0.72}=q_{R}^{2.28}$ for high $q_{R}$, which is a rather strong dependence. For 'variable $q^{\prime} y^{t} / y^{c}$ becomes independent of $q_{R}$ for high $q_{R}$ (Eq. (6.33)), taking the values $1.20 / 0.51=2.35(\mathrm{mf})$ or $2.08 / 0.97=2.14(\mathrm{ev})$. Therefore the liquid window in terms of $y^{t} / y^{c}$ is much narrower when $q$ depends on polymer concentration. For $q_{R}$ close to $q_{R}^{*}$ the difference between the two cases is much smaller: $y^{t} / y^{c} \approx q_{R}^{1.4} / q_{R}^{0.72}=q_{R}^{0.68}$ for 'fixed $q^{\prime}$ and $q_{R}^{1.30} / q_{R}^{0.61}=q_{R}^{0.69}(\mathrm{mf})$ or $q_{R}^{1.55} / q_{R}^{0.72}=q_{R}^{0.83}(\mathrm{ev})$, see Eq. (6.34). We return to the width of the liquid window in chapter 7.

\subsubsection{Critical and triple points}

Fig. 6.12 shows normalized triple points (circles) and critical points (diamonds) for 'variable $q$ ' in the representation $\tilde{y}(f)$. Again the solid curves and closed symbols are for $\mathrm{mf}$, and dashed curves and open symbols are for ev. This figure is analogous to Fig. 5.5 for 'fixed q'. The range for $\tilde{y}(1-5)$ is the same in both figures. Although there are qualitative similarities between the two figures, the quantitative aspects are different. This is most clearly seen in the critical curves. In Fig. 5.5 all the data points are (roughly) in the range $1<\tilde{y}^{c}<3$, and the symbols for $\mathrm{mf}$ and ev lie more or less on a single curve. In contrast, in Fig. $6.12 \tilde{y}^{c}$ exceeds 5 for the highest $q_{R}$ values; the diamonds with the highest $y^{c}$ shown in this figure are for $q_{R}=1.8(\mathrm{ev})$ or 2 ( $\mathrm{mf}$ ). Moreover, the $\mathrm{mf}$ and ev curves no longer coincide. The latter effect is because $\eta^{c}$ (and, hence $f^{c}$ ) is slightly smaller for $\mathrm{mf}$ than for ev, see Eq. (6.35). The difference in the range for $y^{c}$ is due to the different dependence $y^{c}\left(q_{R}\right)$ as discussed in the previous section: $y^{c} \sim q_{R}^{0.72}$ for 'fixed $q^{\prime}$ ', and $y^{c} \sim q_{R}$ (mf) or $y^{c} \sim q_{R}^{1.3}$ (ev) for high $q_{R}$, so the dependence for 'variable $q$ ' is stronger.

Similar quantitative differences occur for $y^{t}$. Here the variation with $q_{R}$ is weaker in Fig. 6.12: at high $q_{R} y^{t} \sim q_{R}^{3}$ for 'fixed $q^{\prime}$ ', and again $y^{t} \sim q_{R}(\mathrm{mf})$ or $y^{t} \sim q_{R}^{1.3}(\mathrm{ev})$ for 'variable $q$ '. The highest triple points shown in Fig. 6.12 are for $q_{R}=1$, whereas in Fig. 5.5 this is $q=0.6$, corresponding roughly to $q_{R}=0.61(\mathrm{mf})$ or $q_{R}=0.66(\mathrm{ev})$.

In Section 6.2 we introduced the parameter $Y=y q_{R}^{-1 / \gamma}$, which has the important property that it approaches a constant value in the

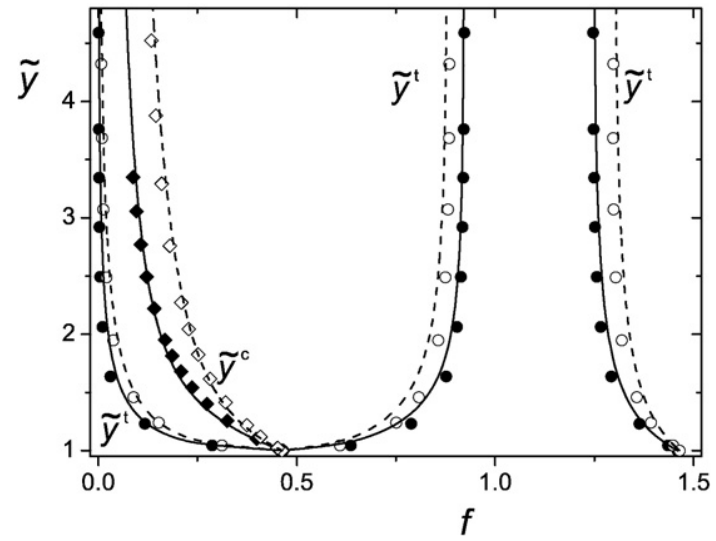

Fig. 6.12. Normalized triple points $\tilde{y}^{t}(f)$ (circles) and critical points $\tilde{y}^{c}(f)$ (diamonds) for 'variable $q$ ', for both $\mathrm{mf}$ (closed symbols) and ev (open). The range for $q_{R}$ is the same as in Fig. 6.2, but the data points for the highest $q_{R}$ values fall outside the range of the figure: the highest triple points shown are for $q_{R}=1$, the highest critical points are for $q_{R}=2(\mathrm{mf})$ and $1.8(\mathrm{ev})$. The curves (solid for $\mathrm{mf}$, dashed for ev) are analytical on the basis of Eq. (6.31) for $y^{t}\left(q_{R}\right)$, Eq. (6.32) for $y^{c}\left(q_{R}\right)$, Eq. (6.35) for $\eta^{c}\left(q_{R}\right)$, and Eqs. (6.36)-(6.38) for the three $\eta^{t}$ 's as a function of $q_{R}$. The $\eta$ 's in these equations were converted to $f$ using $f=\eta /(1-\eta)$.

protein limit. This is illustrated nicely in Fig. 6.13, which replots the data of Fig. 6.12 as $\tilde{Y}^{c}(f)$ and $\tilde{Y}^{t}(f)$. As usual, the symbols are the numerical data and the curves are analytical approximations: Eqs. (6.26) and (6.27) for $Y$, Eqs. (6.35)-(6.38) for $f=\eta /(1-\eta)$. The divergence $y\left(q_{R}\right)$ in Fig. 6.12 disappears and $Y^{c}$ and $Y^{t}$ vary only weakly with $q_{R}$ over the entire range of $q_{R}$, as shown in a different way in Fig. 6.2 and discussed in Section 6.4.1. As in Fig. 6.2, $Y^{t}$ goes up with $q_{R}$ and $Y^{c}$ goes down. The ratio $Y^{t} / Y^{c}$ in Fig. 6.13 is, obviously, the same as $y^{t} / y^{c}$ in Fig. 6.12. The analytical curves in Fig. 6.13 describe the numerical data quite well, with again some deviations for $Y^{c}(f)$ in ev for high $q_{R}$, because Eq. (6.26) neglects the non-monotonic behavior $Y^{c}\left(q_{R}\right)$ which shows up in the numerical data.

\subsection{Phase diagrams in terms of the external polymer concentration $\varphi$}

\subsubsection{Phase diagrams at constant chain length $N$}

For constant $N$ and $\boldsymbol{v}$, the overlap concentration $\varphi_{\mathrm{ov}}$ is constant and independent of $q_{R}$. This implies that $\varphi$ is simply proportional to $y$. When we normalize on the cep the proportionality constant drops out and $\tilde{\varphi}=\varphi / \varphi^{*}$ equals $\tilde{y}$, as also shown in Eq. (5.14). This equation remains valid for 'variable $q$ '. So we may construct $\tilde{\varphi}(\eta)$ diagrams directly from the $y(f)$ diagrams in Figs. 6.10-6.12.

Fig. 6.14 gives such a $\tilde{\varphi}(\eta)$ diagram. The dotted curves are FS binodals for $q_{R}=0.2$ and $q_{R}^{*}$; these are for mf but that hardly matters as

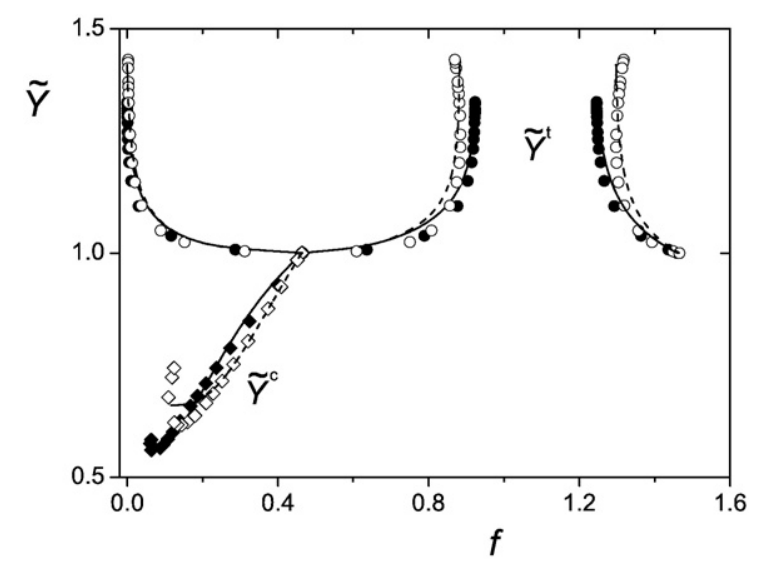

Fig. 6.13. As Fig. 6.12 but now with $\tilde{Y}^{c}$ and $\tilde{Y}^{t}$ along the ordinate axis, and for the complete $q_{R}$ set of Fig. 6.2. 


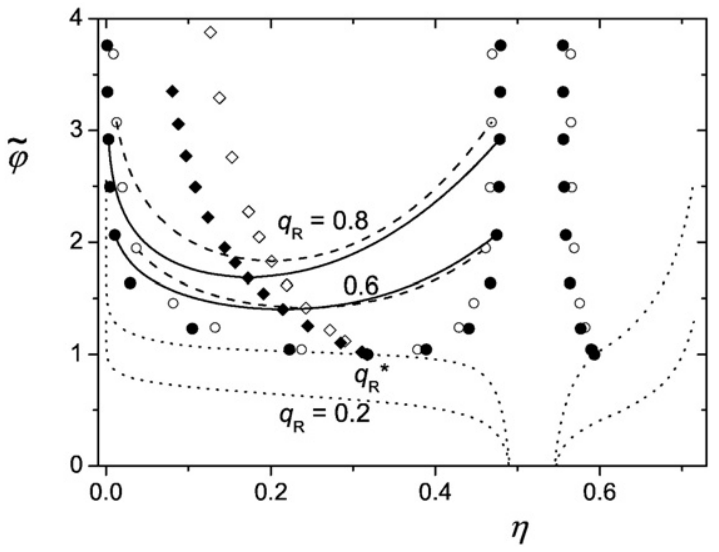

Fig. 6.14. Normalized phase diagrams $\tilde{\varphi}(\eta)$ for 'variable $q$ ' and constant chain length $N$. Triple points (circles) and critical points (diamonds) are the same as in Fig. 6.8. The GL binodals (solid for mf, dashed for ev) are for $q_{R}=0.6$ and 0.8 . The dotted FS binodals are for $q_{R}=q_{R}^{*}$ and 0.2 .

the result for ev would be essentially the same. The solid ( $\mathrm{mf}$ ) and dashed (ev) curves are GL binodals for $q_{R}=0.6$ and 0.8 . The symbols for triple and critical points are the same as in Fig. 6.12 (apart from the conversion from $f$ to $\eta$ ); in this case we do not show the analytical curves of Fig. 6.12.

We may again compare Fig. 6.14 with its 'fixed $q$ ' analogue in Fig. 5.6. Like in the previous Figs. 6.10-6.12 there are qualitative similarities and quantitative differences, which are mainly caused by the different dependence of $y^{c}$ and $y^{t}$ on $q_{R}$ as discussed extensively above.

Fig. 6.15 gives non-normalized triple and critical points for five solvencies and the same five $R$-values as in Fig. 5.7. (These values correspond to $N=400$ with $\nu=3 / 5$ and $\gamma=3 / 4$ as used in chapters 4 and 5; for the present choice $\nu=0.588$ and $\gamma=0.77$ minor deviations occur for ev). The parameter $\varphi$ is obtained by multiplying $\tilde{\varphi}$ in Fig. 6.10 or 11 by $\varphi^{*}=y^{*} \varphi_{\mathrm{ov}}$, where $\varphi_{\mathrm{ov}}$ is given by Eq. (6.3). The overall result is, apart from the quantitative differences as discussed above, the same as in Fig. 5.7 for 'fixed $q$ '. The values of $\varphi^{*}$ and $a^{*}$ corresponding to the cep are given in the legend.

\subsubsection{Phase diagrams at constant particle radius a}

When the particle radius is fixed and $q_{R}$ is changed by varying the radius of gyration, $\varphi_{\text {ov }}$ changes as well: according to Eq. (6.3) $\varphi_{\mathrm{ov}} \sim R^{-1 \gamma}$, where $1 / \gamma$ equals $1(\mathrm{mf})$ or $1.3(\mathrm{ev})$. When we normalize on the cep we find that $\tilde{\varphi}=\tilde{y}$ as used for constant $N$ changes into

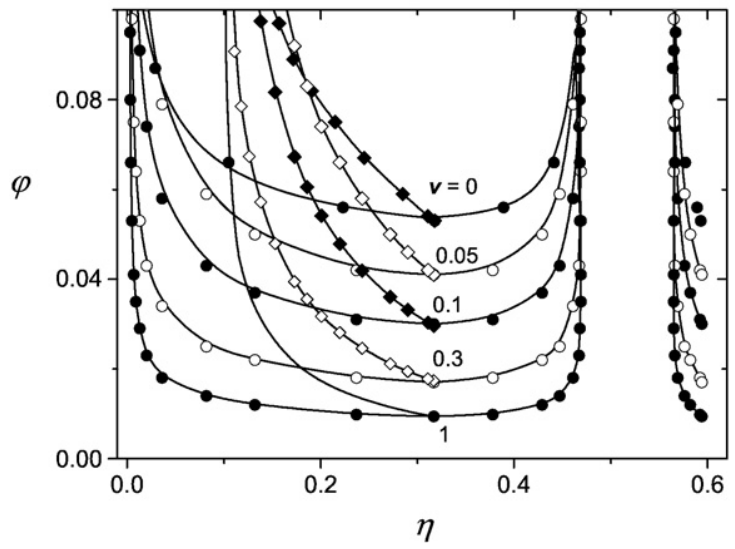

Fig. 6.15. Triple points (circles) and critical points (diamonds) for 'variable $q$ ' in the representation $\varphi(\eta)$, for five solvencies and $R$ values (the same as in Fig. 5.7, corresponding roughly to $N=400$ ). The $q_{R}$ set is the same as in Fig. 6.2. The $\varphi^{*}$ values (Eq. (6.3)) are 0.053, 0.041, 0.030, 0.017, and 0.0094 for $\boldsymbol{v}=0,0.05,0.1,0.3$, and 1 , respectively. The $\alpha^{*}$ values, given by $R / q_{R}^{*}$, are $24.9(\boldsymbol{v}=0), 27.1,29.8,35.5$, and $43.9(\boldsymbol{v}=1)$.

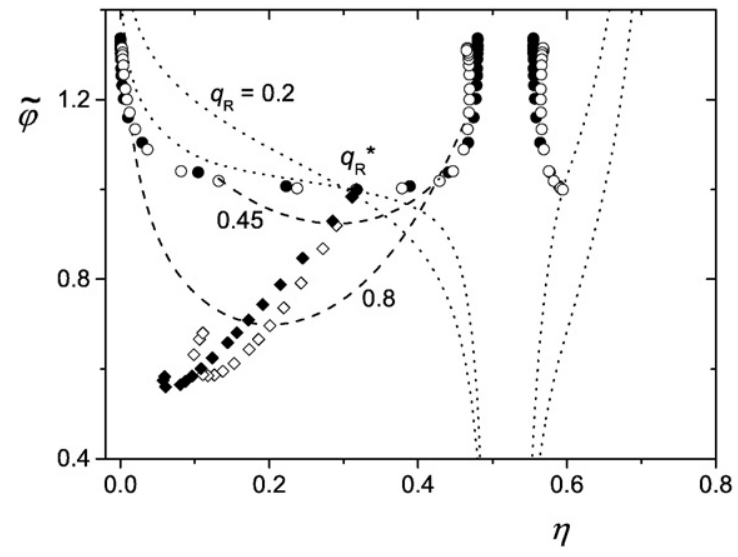

Fig. 6.16. Normalized phase diagram $\tilde{\varphi}(\eta)$ for a constant particle radius $a$ and 'variable $q$ '. Triple points (circles) and critical points (diamonds) are for the same set of $q_{R}$ 's as in Fig. 6.2. Closed symbols are for $\mathrm{mf}$, open ones for ev. The two GL binodals (dashed curves) are for a good solvent and $q_{R}=0.45$ and 0.8 , the two FS binodals (dotted) are for $q_{R}=0.2$ and $q_{R}=q_{R}^{*}$. The FS binodals are for a theta solvent, but those for a good solvent would look essentially the same.

$\tilde{\varphi}=\tilde{y} \tilde{q}_{R}^{-1 / \gamma}=\tilde{Y}$. Hence, Fig. $6.13, \tilde{Y}(f)$ for cp and tp, gives also $\tilde{\varphi}(f)$ for constant $a$. We replot these data in Fig. 6.16 in the form $\tilde{\varphi}(\eta)$, and we add two FS binodals for $\mathrm{mf}\left(q_{R}=0.2\right.$ and $q_{R}^{*}$, dotted $)$ and two GL binodals for ev ( $q_{R}=0.45$ and 0.8 , dashed). This figure may again be compared with its 'fixed $q$ ' analogue in Fig. 5.8. The overall appearance of the two figures is the same, with ascending triple curves, descending critical curves, and crossing FS binodals. This crossing has the same background as in Fig. 5.8, and the inversion was explained in the discussion of this figure.

However, the quantitative differences between Figs. 6.16 and 5.8 are considerable. Whereas in Fig. $5.8 \varphi^{t}$ diverges at high $q_{R}$, in Fig. 6.16 $\tilde{\varphi}^{t}$ reaches a final level around 1.35 (Eq. (6.28b)): even for very high $q_{R}$ $\varphi^{t}$ is only $35 \%$ higher than $\varphi^{*}$. Likewise, the critical points $\tilde{\varphi}^{c}$ reach a final level around 0.6 (Eq. (6.28a)): for high $q_{R} \varphi^{c}$ is only $40 \%$ lower than $\varphi^{*}$. For high $q_{R}$ the width of the liquid window in terms of $\varphi^{t} / \varphi^{c}$ is constant, roughly $1.35 / 0.6=2.3$. A liquid thus exists only over a narrow range of polymer concentrations. We return to this point in chapter 7 .

The normalized plot $\tilde{\varphi}(\eta)$ in Fig. 6.16 may be converted into a nonnormalized plot $\varphi(\eta)$ by multiplying $\tilde{\varphi}$ by $\varphi^{*}=y^{*} \varphi_{\mathrm{ov}}$, where $\varphi_{\mathrm{ov}}$ is given by a slightly modified version of Eq. (5.15): $\varphi^{*}=1.48 \mathrm{a}^{-1}(\mathrm{mf})$ or $\varphi^{*}=1.47 \boldsymbol{v}^{-0.28} a^{-1.3}(\mathrm{ev})$. Fig. 6.17 gives this plot $\varphi(\eta)$ for $a=30$ and (from top to bottom) $\boldsymbol{v}=0,0.05,0.1,0.3$, and 1 , corresponding to $\varphi^{*}=0.043,0.035,0.029,0.021$, and 0.015 , respectively. We obtain five

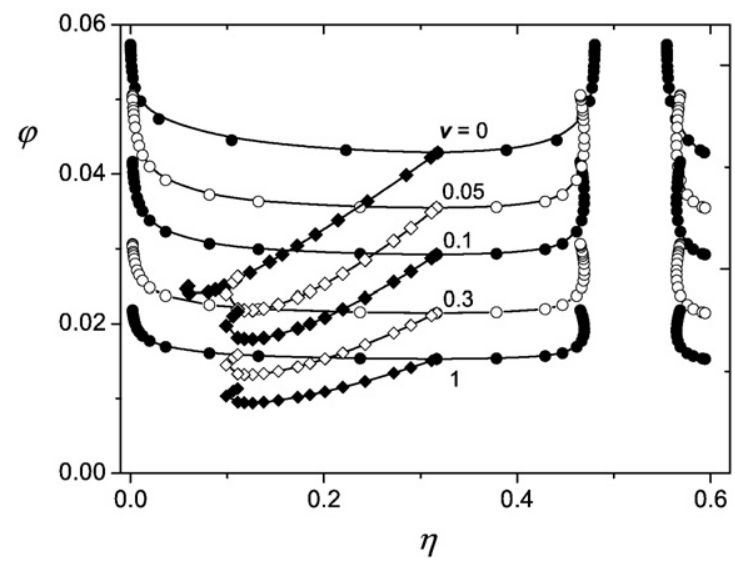

Fig. 6.17. Triple points (circles) and critical points (diamonds) for $a=30$ and five solvencies (the same set as in Fig. 6.15), in the representation $\varphi(f)$ and for 'variable $q$ '. The $q_{R}$ set is the same as in Fig. 6.2. The $\varphi^{*}$ values are $0.043(\boldsymbol{v}=0), 0.035,0.029,0.021$, and $0.015(\boldsymbol{v}=1)$. The $R^{*}$ values are given by $a q_{R}^{*}$ : $10.1(\mathrm{mf})$ and $11.6(\mathrm{ev})$. 


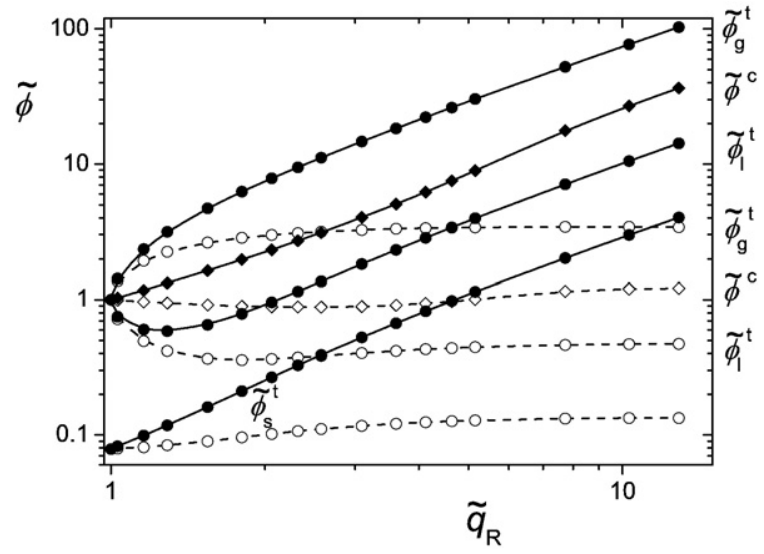

Fig. 6.18. Normalized internal concentrations $\tilde{\phi}^{c}$ (cp, diamonds) and $\tilde{\phi}^{t}$ (tp, circles) as a function of $\tilde{q}_{R}$ for 'variable $q$ ' and a good solvent, on a double-logarithmic scale. Closed symbols and solid curves are for constant $N$, open symbols with dashed curves are for constant $a$; the symbols correspond to the standard set of $q_{R}$ 's (see Fig. 6.2). The normalized $\tilde{\phi}$-values may be converted to real concentrations $\phi$ by multiplying with $\phi^{*}=\alpha^{*} \varphi^{*}$, where $\varphi^{*}=y^{*} \varphi_{\mathrm{ov}}$, with $\varphi_{\mathrm{ov}}$ given by $0.87 \boldsymbol{v}^{-0.28} R^{-1.3}$ (constant $N$ ) or 1.47 $\boldsymbol{v}^{-0.28} a^{-1.3}$ (constant $a$ ).

duplicates of Fig. 6.16 (with only tp and cp shown in Fig. 6.17), differing only in the value of $\varphi^{*}$. Like in Fig. 6.16 each duplicate covers the range of (roughly) $0.6 \varphi^{*}<\varphi<1.4 \varphi^{*}$, which is a much narrower range than in the analogous Fig. 5.9 for 'fixed q'.

\subsection{Phase diagrams in terms of the internal concentration $\phi$}

\subsubsection{Critical and triple points in internal concentrations}

Fig. 6.18 illustrates how the internal polymer concentrations $\phi=\alpha \varphi$ in critical point (diamonds) and triple point (circles, three branches) depend on $q_{R}$. This figure is for a good solvent, and gives the normalized concentrations $\tilde{\phi}^{c}=\phi^{c} / \phi^{*}, \tilde{\phi}_{g}^{t}=\phi_{g}^{t} / \phi^{*}, \tilde{\phi}_{l}^{t}=\phi_{l}^{t} / \phi^{*}$, and $\tilde{\phi}_{\mathrm{s}}^{t}=\phi_{\mathrm{s}}^{t} / \phi^{*}$ as a function of $\tilde{q}_{R}=q_{R} / q_{R}^{*}$ on a double-logarithmic scale, for both constant chain length (closed symbols, solid curves) and constant particle radius (open symbols, dashed curves). Fig. 6.18 for 'variable q' may be compared with Fig. 5.12 for 'fixed $q$ '. There are some similarities, but also important quantitative differences. For the liquid and solid branches of the triple curve the behavior is qualitatively different: in Fig. 5.12 the internal concentrations for these branches decay rather fast to very small values, whereas in Fig. 6.18 these concentrations are more or less constant (for constant a) or even increase with $q_{R}$ (constant $N$ ).

We first discuss the dashed curves for constant $a$. According to Eq. (6.28) the external concentrations $\tilde{\varphi}=\tilde{Y}$ reach a constant level $\left(\tilde{\varphi}^{c}=0.66, \tilde{\varphi}^{t}=1.42\right)$ for large $q_{R}$. Also the $\alpha^{\prime}$ s reach such a constant level. For $\tilde{\alpha}^{c}$ this level is 1.80 (Eq. (6.40)), so $\tilde{\phi}=1.80 \cdot 0.66=1.19$ for high $q_{R}$. Therefore $\tilde{\phi}^{C}$ varies only weakly with $q_{R}$, from 1 in the cep to 1.19 for high $q_{R}$. The fact that for $\tilde{q}_{R}$ close to $1 \tilde{\phi}^{c}$ drops slightly below unity is easily understood: close to the cep $y^{c} \sim q_{R}{ }^{0.72}$ (Eq. (6.34)) so $\varphi^{c} \sim \mathrm{y}^{c} q_{R}{ }^{-1.3} \sim$ $q_{R}^{-0.58}$ and $\alpha^{c} \sim q_{R}^{0.33}$ (Eq. (6.40)), so $\tilde{\phi}^{c} \sim q_{R}^{-0.25}$, giving an initial decrease of $\phi^{c}$ with increasing $q_{R}$.

For the gas branch of the triple curve the final level for $\alpha_{g}^{t}$ is 1 (Eq. (6.42)), so $\tilde{\alpha}_{g}^{t}=1 / \alpha^{*}=2.62$ and $\tilde{\phi}_{g}^{t}=2.62 \cdot 1.42=3.72$. For the liquid branch $\alpha_{l}^{t}=0.118$ (Eq. (6.44)) or $\tilde{\alpha}_{l}^{t}=0.31$ for high $q_{R}$ so $\tilde{\phi}_{l}^{t}=$ $0.31 \cdot 1.42=0.44$. In these two branches $\tilde{\phi}^{t}\left(q_{R}\right)$ goes gradually from unity in the cep to these final values (with a weak minimum in the liquid branch). For the solid branch the high- $q_{R}$ limit is $\alpha_{s}^{t}=0.042$ (Eq. (6.45)) so that $\tilde{\phi}_{s}^{t}=(0.42 / 0.38) \cdot 1.42=0.16$, and $\tilde{\phi}_{s}^{t}$ increases from $\alpha_{\mathrm{s}}^{*} / \alpha^{*}=0.079$ in the cep to 0.16 for high $q_{R}$. The fact that for 'variable $q$ ' both $\alpha^{t}$ and $\phi^{t}$ reach a final level explains the qualitatively different behavior in the liquid and solid branches of the triple curves in Fig. 6.18 as compared to Fig. 5.12: in the latter figure $\alpha^{t}$ and $\phi^{t}$ go to zero for high $q_{R}$.
Whereas the dashed curves for constant $a$ in Fig. 6.18 reach a constant level, the solid curves for constant $N$ behave as a power law $\phi \sim q_{R}{ }^{1 / \gamma}=q_{R}{ }^{1.30}$ for high $q_{R}$. The reason is, obviously, the prefactor $\tilde{q}_{R}^{1.30}$ in Eqs. (6.31) and (6.32) for $\tilde{\phi}^{t}=\tilde{y}^{t}$ and $\tilde{\phi}^{c}=\tilde{y}^{c}$. This prefactor gives a stronger dependence of $\phi^{t}$ and $\phi^{c}$ for constant $N$ as compared to constant $a$, a feature which shows up also in Figs. 6.14 and 6.15 (constant $N$ ) as compared to Figs. 6.16 and 6.17 (constant $a$ ).

Fig. 6.18 is for a good solvent. We do not give the analogous figure for a theta solvent as this is very similar, apart from some minor quantitative differences. These are related to the slightly different final levels for $\alpha^{c}$ and $\alpha^{t}$ (see Eqs. (6.40), (6.44) and (6.45)) and for $\tilde{\phi}^{c}=\tilde{Y}^{c}$ and $\tilde{\phi}^{t}=\tilde{Y}^{t}$ for constant $a$ Eq. (6.28). For constant $N$ an additional difference shows up in the prefactor $\tilde{q}_{R}^{1 / \gamma}$ in Eqs. (6.31) and (6.32), since $\gamma=1$ in a theta solvent.

\subsubsection{Phase diagrams at constant chain length $N$}

Fig. 6.19 gives a normalized phase diagram $\tilde{\phi}(\eta)$ for a good solvent and constant $N$, with all the triple points and critical points with $\tilde{\phi}$ below 5 , one FS binodal (for $q_{R}=0.2$, dotted), three GL binodals $\left(q_{R}=0.45,0.5\right.$, and 0.6 , dashed), and the triple triangle for $q_{R}=0.6$. This figure for 'variable $q$ ' is fully comparable with Fig. 5.13 for 'fixed $q$ ' but there are clear quantitative differences, especially in the critical curve and in the $\mathrm{L}$ and $\mathrm{S}$ branches of the triple point. These are related to the different dependence of $\phi^{c}$ and $\phi^{t}$ on $q_{R}$, as discussed in the previous section. The critical curve $\phi^{c}\left(q_{R}\right)$ goes up more steeply for 'variable $q^{\text {', }}$ and the liquid and solid branches of the triple curve go up with $q_{R}$ for 'variable $q$ ' (Fig. 6.19), whereas they go down for 'fixed $q$ ' (Fig. 5.13). The highest critical point shown in Fig. 6.19 is for $q_{R}=1.2$, and for the triple curve the highest points are for $q_{R}=0.6$ (gas branch), $q_{R}=2$ (liquid branch), and $q_{R}=5$ (solid branch).

The increase of $\varphi^{t}=\alpha^{t} y^{t}$ with $q_{R}$ in the three branches of the triple curve in Fig. 6.19 is easily understood: for high $q_{R}$ we have $\varphi^{t} \sim y \sim q_{R}^{1.3}$ since $\alpha^{t}$ becomes constant $(1,0.12$, and 0.04 in the gas, liquid, and solid branches, respectively). The liquid branch goes through a minimum because close to the cep $\varphi_{l}{ }^{t}$ decreases with $q_{R}$ : here the decrease of $\varphi_{l}^{t}$ with increasing $q_{R}$ (see Eq. (6.44) and Fig. 6.9) is stronger than the increase of $y^{t}\left(\sim q_{R}^{1.55}\right.$ according to Eq. (6.34) and Fig. 6.6).

\subsubsection{Phase diagrams at constant particle radius a}

Fig. 6.20 gives a similar phase diagram for constant $a$, and is the 'variable $q$ ' counterpart of Fig. 5.14 for 'fixed $q$ '. On first sight the differences between these two figures is less than in Figs. 5.13 and 6.19 for constant $N$. However, the effect of $q_{R}$ is considerably smaller in

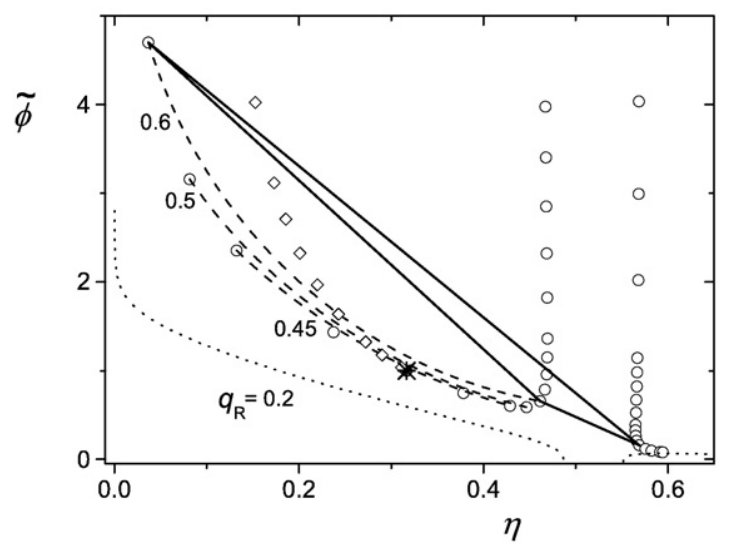

Fig. 6.19. Normalized phase diagram $\tilde{\phi}(\eta)$ for a constant chain length $N$ and 'variable $q$ ' in a good solvent. The critical points (diamonds) and triple points (circles) are for the standard set for $q_{R}$ (see Fig. 6.2), but not all these points are in the range shown: the highest critical point is for $q_{R}=1.2$, the highest triple points are for $q_{R}=0.6(\mathrm{G}), q_{R}=2(\mathrm{~L})$, and $q_{R}=5(\mathrm{~S})$. Three GL binodals $(q=0.45,0.5,0.6)$ are indicated as the dashed curves. The asterisk is the cep at $\tilde{\phi}=1, \eta=\eta^{*}$. The triple triangle connects the three $\tilde{\phi}^{t}, \eta^{t}$ pairs for $q_{R}=0.6$. The dotted curve is the FS binodal for $q_{R}=0.2$. 


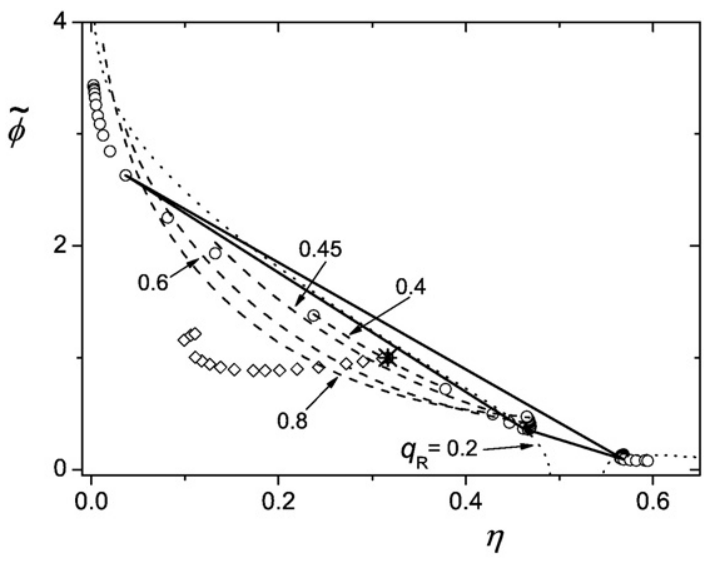

Fig. 6.20. Normalized phase diagram $\tilde{\phi}(\eta)$ for a constant particle radius $a$ and a good solvent. Four GL binodals $\left(q_{R}=0.4,0.45,0.6,0.8\right)$ are indicated as the dashed curves. Triple points (circles) and critical points (diamonds) are for the standard set (see Fig. 6.2) for $q_{R}$. The asterisk is the cep at $\tilde{\phi}=1, \eta=\eta^{*}$. The triple triangle connecting the three $\tilde{\phi}^{t}, \eta^{t}$ pairs is for $q_{R}=0.6$. The dotted curve is the FS binodal for $q_{R}=0.2$.

Fig. 6.20, and all the critical and triple points, up to $q_{R}=5$, are shown in this figure. Again the FS binodal (dotted) is for $q_{R}=0.2$, and four GL binodals $\left(q_{R}=0.4,0.45,0.6,0.8\right)$ are given as the dashed curves. The triple triangle is for $q_{R}=0.6$.

We see from Fig. 6.20 that the entire GL binodals for any $q_{R}$ lie in a rather narrow range. For high $q_{R}$ this range is roughly $4 \phi^{*}>\phi>\phi^{*}$ for the gas branch, and $\phi^{*}>\phi>0.5 \phi^{*}$ for the liquid branch. This indicates that also in terms of the internal concentrations the liquid window is narrow, as will be discussed in more detail in the next chapter.

\subsection{Analytical GL binodals}

In the previous sections we found analytical approximations for the three characteristic points of a GL binodal: the critical point $y^{c}, \eta^{c}$ and the gas and liquid parts of the triple point $y^{t}, \eta_{g}^{t}$ and $y^{t}, \eta_{l}^{t}$. So far, we did not consider an analytical form of a binodal $y(\eta)$ for the region $y^{c}<y<y^{t}, \eta_{g}^{t}<\eta<\eta_{l}^{t}$; this we do in the present section. It turns out that analytical approximations for binodals $Y(\eta)$ are easier; from those the binodals $y(\eta)$ follow then from $y=Y q_{R}^{1 / \gamma}$.

For analytical approximations for GL binodals $Y(\eta)$, we treat the $\mathrm{L}$ and G branches separately. In Section 3.2.3 we found that for 'fixed $q$ ' the liquid branch could be well described by an equation of the type

$$
\Delta \Pi v / \mathrm{D} \Pi v=(\Delta f / \mathrm{D} f)^{x} \quad \Delta X=X-X^{c} \quad \mathrm{D} X=X^{t}-X^{c}
$$

where $f=\eta /(1-\eta)$ represents the colloid concentration and $\mathrm{D} f=f_{l}^{t}-f^{c}$. For 'fixed $q$ ' the exponent $x$ was found to be 2, so that the binodal $\Pi v$ $(f)$ is a parabola. Note that both $\Delta \Pi v / \mathrm{D} \Pi v$ and $\Delta f / \mathrm{D} f$ run from zero in the $\mathrm{cp}$ (lowest point of the parabola) to unity in the tp (highest point).

We have also seen (Fig. 6.4) that the parameter $\Pi v$ is nearly the same for 'fixed $q$ ' and 'variable $q$ '. It is then reasonable to try a similar procedure for a binodal $\Pi v(\eta)$ for 'var'. We will show that such an approximation does indeed work. The binodal $\Pi v(\eta)$ may be converted to the binodal $\mathrm{Y}(\eta)$ using Eq. (6.18). When $\eta$ is chosen and $\Pi v$ is calculated from it, the conversion to $Y$ requires solving an implicit equation. It is easier to invert the procedure: choose a value for $Y$ in the range $Y^{c}<Y<Y^{t}$, calculate the corresponding $\Pi v$ directly from Eq. (6.18), and compute $\eta$ from an appropriate binodal equation $\Delta \eta \sim(\Delta \Pi v)^{1 / x}$. It turns out that for 'var' the following equation describes the L branch quite satisfactory for any $q_{R}$ and for both $\mathrm{mf}$ and ev:

$$
\Delta \eta / \mathrm{D} \eta=(\Delta \Pi v / \mathrm{D} \Pi v)^{0.4} .
$$

Figs. $6.21(\mathrm{mf})$ and $6.22(\mathrm{ev})$ show the comparison in the representation $Y(\eta)$. Symbols are numerical results, curves are

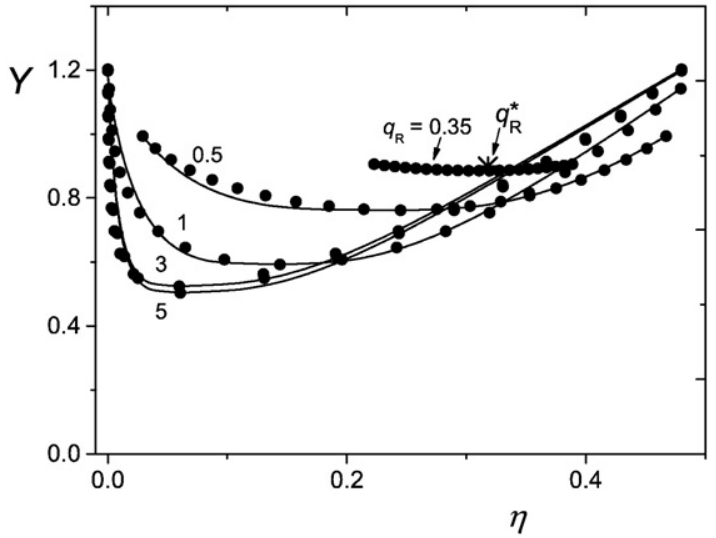

Fig. 6.21. GL binodals $Y(\eta)$ for $\mathrm{mf}$ and $q_{R}=0.35,0.5,1,3$ and 5 . Symbols are the numerical data, curves are the analytical approximations of Eq. (6.47) (L branch) and Eq. (6.48) ( $\mathrm{G}$ branch). The asterisk is the cep.

analytical; for the L branches Eq. (6.47) was used. The asterisk in these figures is the cep at $Y=Y^{*}, \eta=\eta^{*}$ (point binodal). For $q_{R}$ slightly above $q_{R}^{*}(0.35$ in Fig. 6.21, 0.4 in Fig. 6.22) the binodal is very flat and spans only a very narrow range in $Y$ and a somewhat wider range in $\eta$. For $q_{R}=0.5$ and $q_{R}=1$ the range is wider: for $\eta$ it approaches the entire interval $0<\eta<\eta_{f}^{0}$, but for $Y$ it is (for $q_{R}=1$ or above) only a factor of roughly 2 . This is in line with our earlier results for binodals $\tilde{\varphi}(\eta)=$ $\tilde{Y}(\eta)$ for constant $a$ (Figs. 6.16 and 6.17). For the two highest $q_{R}$ values in Figs. 6.21,6.22 we are in the protein limit: the entire GL binodal $Y$ $(\eta)$ is independent of $q_{R}$ and $Y^{t} / Y^{c}$ equals $1.20 / 0.51=2.35$ for $\mathrm{mf}$ and $2.08 / 0.97=2.14$ for ev according to Eq. (6.28). We conclude from Figs. 6.21 and 6.22 that the analytical Eq. (6.47) for the liquid branch works quite well, for any $q_{R}$.

The gas branch is not well described by Eq. (6.47) because for small $\eta$ the binodal is quite steep. In Section 3.2.3 we modelled this part using the exclusion limit for 'fixed $q$ '. Here we try a different procedure and we do not consider $\Pi \mathrm{v}(\eta)$ as in Eq. (6.47) but $q(\eta)$, where the relation between $q$ and $Y$ is given in Eq. (6.17). The analytical $G$ branches in Figs. 6.21 and 6.22 were computed from

$\Delta \eta / \mathrm{D} \eta=(\Delta q / \mathrm{D} q)^{0.25}$.

Note that now $\Delta \eta=\eta^{c}-\eta, \mathrm{D} \eta=\eta^{c}-\eta_{\mathrm{g}}^{t}\left(\approx \eta^{c}\right.$ for high $\left.q_{R}\right), \Delta q=q^{c}-q$, and $\mathrm{D} q=q^{c}-q^{t}\left(\approx 0.40\right.$ for high $q_{R}$ in $\mathrm{mf}$, or $\left.0.19 \mathrm{in} \mathrm{ev}\right)$. The agreement between Eq. (6.48) (converted to $\eta(Y)$ binodals) and the numerical data is not perfect for intermediate $q_{R}$ but the trends are captured quite well and the steep part of the gas branch is reasonably described.

It is instructive to consider two features of the analytical binodals of Eqs. (6.47) and (6.48) in somewhat more detail: the slope $d Y / d \eta$ at

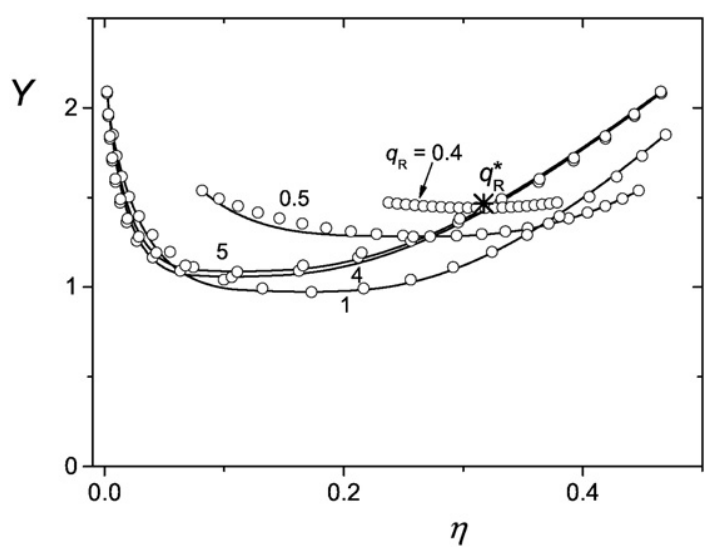

Fig. 6.22. As in Fig. 6.21 but now for ev and $q_{R}=0.4,0.5,1,4$, and 5 . 
both endpoints (near tp) and the behavior around the cp. The former issue is related to the asymmetry of the two branches, the latter gives information about the critical exponent $x$ in $\Delta \eta \sim(\Delta y)^{x}$ or $\Delta \eta \sim(\Delta Y)^{x}$.

The slope $d Y / d \eta$ can be written as $(d \Pi v / d \eta) /(d \Pi v / d Y)$ (where we use Eqs. (6.47) and (6.19)) or as $(d q / d \eta) /(d q / d Y)$ (with Eqs. (6.48) and (6.17)). Clearly, $d \Pi v / d \eta$ equals $d \Delta \Pi v / d \Delta \eta$ : from Eq. (6.47) we get $d \Pi v /$ $d \eta=2.5(\mathrm{D} \Pi v / \mathrm{D} \eta)(\Delta \eta / \mathrm{D} \eta)^{1.5}$, which at tp simplifies to $d \Pi v / d \eta=2.5$ $(\mathrm{D} \Pi v / \mathrm{D} \eta)$. From Eq. (6.19) (protein limit) we have $d \Pi v / d Y=3 \gamma c_{2} Y^{3 \gamma-1}$, where we substitute $Y=Y^{t}$. The result at the top of the liquid branch is:

$\frac{d Y}{d \eta}=\frac{2.5 Y^{t}}{3 \gamma} \frac{1-\left(Y^{c} / Y^{t}\right)^{3 \gamma}}{\eta_{l}^{t}-\eta^{c}} \quad\left(Y=Y^{t}\right.$, high $\left.q_{R}\right)$.

Inserting the appropriate analytical values for $Y^{t}, Y^{c}, \eta_{l}^{t}$, and $\eta^{c}$ we find $d Y / d \eta=2.3$ (mf) or $5.2(\mathrm{ev})$, which values agree with the final L-branch slopes in Figs. 6.21 and 6.22 for the protein limit.

In a similar way we obtain the initial G-branch slope:

$\frac{d Y}{d \eta}=-\frac{4 Y^{t}}{\tau} \frac{\left(Y^{t} / Y^{c}\right)^{\tau}-1}{\eta^{c}-\eta_{g}^{t}} \quad\left(Y=Y^{t}\right.$, high $\left.q_{R}\right)$

where $\tau(\approx 0.9 \gamma)$ is the protein-limit exponent in $q \sim Y^{-\tau}$. Eq. (6.50) gives a much steeper G-branch slope $d Y / d \eta=-84(\mathrm{mf})$ or $-102(\mathrm{ev})$ in the protein limit. This analysis shows that using the parameter $\Pi v$ in the $\mathrm{L}$ branch and the parameter $q$ in the $\mathrm{G}$ branch automatically leads to asymmetric binodals.

Next we consider the region around the $\mathrm{cp}$, where the binodals are very flat. When $\Delta Y$ is small and in the protein limit $\left(\Pi v=A Y^{3 \gamma}, q=B Y^{-\tau}\right)$ we may write $\Delta \Pi v=\Pi v-(\Pi v)^{c}$ as ${ }_{0} A^{0}\left\{\left(Y^{c}+\Delta Y\right)^{3 \gamma}-\left(Y^{c}\right)^{3 \gamma}\right\} \approx$ $3 \gamma A\left(Y^{c}\right)^{3 \gamma-1} \Delta Y$ and $\Delta q=q^{c}-q$ as $B\left\{\left(Y^{c}\right)^{-\tau}-\left(Y^{c}+\Delta Y\right)^{-\tau}\right\} \approx \tau B\left(Y^{c}\right)^{-\tau-1} \Delta Y$. Hence, around the cp $\Delta Y$ is proportional to $\Delta \Pi v$ (L branch) or to $\Delta q(\mathrm{G}$ branch). Then according to Eqs. (6.47) and (6.48) $\Delta \eta \sim(\Delta Y)^{0.4}$ (L branch) or $\Delta \eta \sim(\Delta Y)^{0.25}$ (G branch). In our primitive approach the critical exponents are not the same at both sides of the cp. However, both exponents are smaller than the 'fixed $q$ ' value 0.5 . In recent simulations [56] a critical exponent around 0.33 was found, which happens to be the average of our two values.

It would have been possible to describe the liquid branch of the GL binodal as a power law not in the parameter $\Pi v$, but directly in Y: $\Delta \eta / \mathrm{D} \eta=(\Delta Y / \mathrm{DY})^{x}$. This works excellently for given $q_{R}$, but when $q_{R}$ is varied the exponent $x$ has to be adjusted for obtaining a good fit. In that respect Eq. (6.47), with an exponent which is independent of $q_{R}$ (and is the same for $\mathrm{mf}$ and $\mathrm{ev}$ ) is more elegant. Apparently, the $q_{R}$-dependence in the conversion between $Y$ and $\Pi v$ is captured sufficiently well by Eq. (6.18). For the gas branch a power law $\Delta \eta \sim(\Delta Y)^{x}$ does not work at all

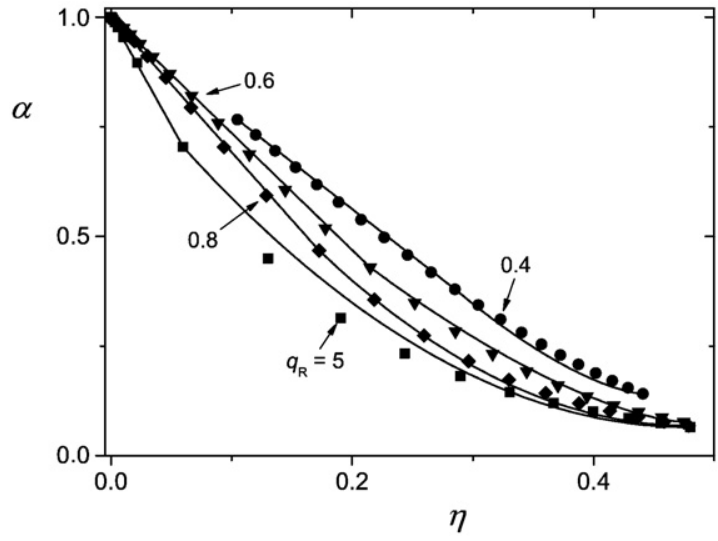

Fig. 6.23. The free volume fraction $\alpha$ along the binodals for a theta solvent and $q_{R}=0.4$, $0.6,0.8$, and 5. Symbols are the numerical data, curves are Eq. (6.51) (G branch) and Eq. (6.52) (L branch). The exponent $x$ in Eq. (6.52) is 1.5 for $q_{R}=0.4$ and 0.6 , and 2 for $q_{R}=0.8$ and 5 .

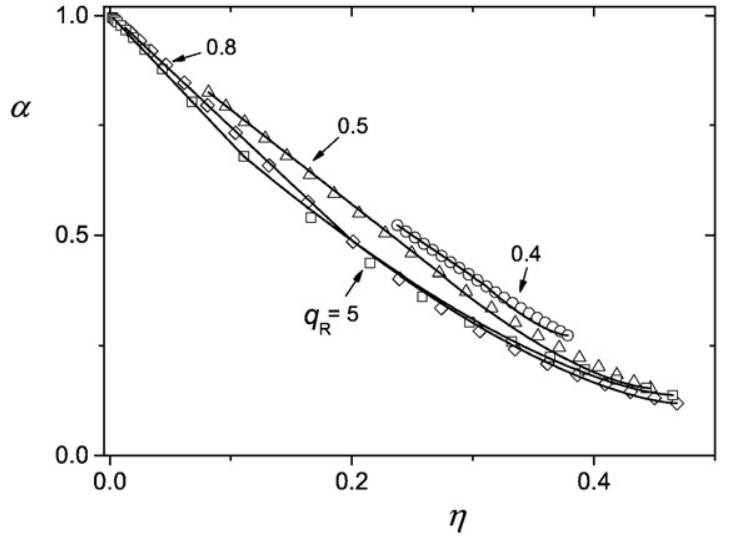

Fig. 6.24. As Fig. 6.23 but now for a good solvent and $q_{R}=0.4,0.5,0.8$, and 5 , with $x=1.5$ (L branch).

for small $\eta$, whereas Eq. (6.48) with a power law in $\Delta q$ describes the numerical data reasonably well, as shown above.

In order to convert the 'external' binodals to 'internal' binodals we have to multiply $y$ or $Y$ with the free volume fraction $\alpha=\alpha(\eta, q)$ (Eq. (2.5)), where $q$ is given by Eq. (6.17). In principle, this is straightforward as $q=q(y)$ is known for any $\eta$ between $\eta_{g}^{t}$ and $\eta_{l}^{t}$. However, the form of Eq. (2.5) and of $q(\eta)$ makes the dependence $\alpha(\eta)$ rather complicated. When the numerical $\alpha$ s are plotted as a function of $\eta$ (see Figs. 6.23 and 6.24) the gas branch is essentially a straight line for any $q_{R}$ and both for $\mathrm{mf}$ and ev. It is given by

$\mathrm{G} \quad \Delta \alpha / \mathrm{D} \alpha=\Delta \eta / \mathrm{D} \eta$

where $\Delta \alpha=\alpha_{g}^{t}-\alpha, \mathrm{D} \alpha=\alpha_{g}^{t}-\alpha^{c}, \Delta \eta=\eta-\eta_{g}^{t}$, and $\mathrm{D} \eta=\eta^{c}-\eta_{\mathrm{g}}^{t}$. The background of this linearity is again Eq. (2.2): $\alpha=1-\eta(1+q)^{3}$, where $q$ is small and does not vary much in the gas branch.

The liquid branch is not linear, and can be described as a power law

$\mathrm{L} \quad \Delta \alpha / \mathrm{D} \alpha=(\Delta \eta \mathrm{D} \eta)^{X}$

with $\Delta \alpha=\alpha-\alpha_{l}^{t}$, D $\alpha=\alpha^{c}-\alpha_{l}^{t}, \Delta \eta=\eta_{l}^{t}-\eta$, and $\mathrm{D} \eta=\eta_{l}^{t}-\eta^{c}$.

It turns out that $x=1.5$ gives a rather accurate approximation for ev (any $q_{R}$ ) and for $\mathrm{mf}$ for relatively low $q_{R}$ (0.6 and below). For the $\mathrm{mf}$ binodals for higher $q_{R}$ an exponent $x=2$ works better. The curves in Fig. 6.23 (mf) and 6.24 (ev), drawn according to Eqs. (6.51) and (6.52) with this exponent $x$, show that these simple equations describe the numerical data satisfactorily (though not perfect for mf and very high $q_{R}$ ).

In chapter 8 we shall use the analytical equations given above to interpret experimental GL binodals.

\section{The liquid window}

Liquid is only stable between the critical point (cp) and the triple point (tp) and for $q_{R}>q_{R}^{*}$, where $q_{R}^{*}$ corresponds to the critical endpoint (cep). In this chapter we analyze the liquid window in terms of external and internal polymer concentrations and in terms of the contact potential $\varepsilon$ between two colloids in a solution of nonadsorbing polymer, both for 'fixed $q$ ' (i.e., ideal osmotic pressure, concentration-independent $q$ ) and for 'variable $q$ ' (non-ideal osmotic pressure, concentration-dependent $q$ ). We restrict ourselves to polymer in a good solvent (ev, excluded-volume chains). We employ a fully analytical description, using previously derived analytical approximations to the numerical results for 'fix' and 'var'. As we have seen before, these approximations are not perfect but they describe the trends quite well and may form a useful guideline for experimentalists to quantitatively describe measured phase lines.

In Section 7.1 we summarize the most important equations, whereby we express all quantities in the size ratio $q_{R}=R / a$. The size 
ratio $q=\delta / a$, which may be seen as the relative range of the interaction between two colloids, is a function of $q_{R}$ and the (normalized) polymer concentration $y=\varphi / \varphi_{\mathrm{ov}}$. For the critical and triple points, $y^{c}$ and $y^{t}$ are known functions of $q_{R}$, so $q^{c}$ and $q^{t}$ may be expressed as a function of $q_{R}$ only.

In order to quantify the conditions for a stable liquid we discuss the strength $\varepsilon$ of the interaction in Section 7.2. This strength is of order $\varepsilon \approx \Pi v_{\mathrm{ov}}$, where $v_{\mathrm{ov}}$ is the overlap volume of the depletion layers of two particles in contact. We will see that a more accurate approximation is $\varepsilon \approx v_{\text {ov }} y d \Pi / d y$.

In Section 7.3 we present analytical results for the liquid window. We discuss the liquid window in terms of the strength (Section 7.3.1), in terms of the external polymer concentrations $\varphi$ (Section 7.3.2), and in terms of the internal concentrations $\phi$ (Section 7.3.3). In all cases we compare 'fix' and 'var'. In addition, in Section 7.3.1 we make a comparison with the liquid window for a quite different system: a one-component hard-sphere system with a Yukawa-type attraction.

\subsection{Summary of equations for critical and triple points}

We have found before (Eqs.(5.4), (5.9), (5.17) and (6.20), (6.21), (6.23)) the coordinates of the critical endpoint:

$q_{R}^{*}=\left\{\begin{array}{l}0.330 \\ 0.388\end{array} \quad y^{*}=\left\{\begin{array}{l}0.385 \\ 0.428\end{array} \quad \alpha^{*}= \begin{cases}0.317 & \text { fix } \\ 0.381 & \text { var }\end{cases}\right.\right.$

The indications 'fix' and 'var' are abbreviations for 'fixed $q$ ' and 'variable $q$ ', respectively.

We have also seen (Eqs. (5.10), (5.11) and (6.31), (6.32)) that $y^{c}$ for the critical point and $y^{t}$ for the triple point are most easily formulated in terms of the reduced parameters $\tilde{y}=y / y^{*}$ and $\tilde{q}_{R}=q_{R} / q_{R}^{*}$ :

$\tilde{y}^{c} \approx \begin{cases}\tilde{q}_{R}^{0.72} & \text { fix } \\ \tilde{q}_{R}^{1.3}\left(0.66+0.34 e^{1.7\left(1-\tilde{q}_{R}\right)}\right) & \text { var }\end{cases}$

$\tilde{y}^{t}=\left\{\begin{array}{ll}\tilde{q}_{R}^{3}\left(0.567+0.433 \tilde{q}_{R}^{-3.7}\right) & \text { fix } \\ \tilde{q}_{R}^{1.3}\left(1.42-0.42 e^{0.6\left(1-\tilde{q}_{R}\right)}\right) & \text { var }\end{array}\right.$.

The high- $q_{R}$ limits in Eqs. (7.2b) and (7.3) follow immediately by omitting the second term. As discussed before, close to the cep $\tilde{y}=\tilde{q}_{R}^{\lambda}$, with $\lambda=0.72$ (Eq. (6.34)) in Eq. (7.2b), $\lambda=1.4$ (Eq. (5.11)) in Eq. (7.3a), and $\lambda=1.55$ (Eq. (6.34)) in Eq. (7.3b).

The parameter $\tilde{y}$ may be converted to the parameter $\tilde{\varphi}$ by taking into account the variation of $\varphi_{\mathrm{ov}}$ in $y=\varphi / \varphi_{\mathrm{ov}}$ with $q_{R}$. For fixed polymer radius $R, \varphi_{\mathrm{ov}}$ is constant (or $\tilde{\varphi}_{\mathrm{ov}}=1$ ). For fixed particle radius $a, \varphi_{\mathrm{ov}}$ is proportional to $q_{R}^{-1 / \gamma}$ (with $\gamma=0.77$ ):

$\tilde{\varphi}= \begin{cases}\tilde{y} & \text { constant } R \\ \tilde{y} \tilde{q}_{R}^{-1.3}=\tilde{Y} & \text { constant } a\end{cases}$

We need also the relation between the parameters $q=\delta / a$ and $q_{R}=$ $R / a$. For 'fixed $q$ ' this is independent of the polymer concentration (Eq. (5.5)), for 'variable $q$ ' the depletion layers are compressed by the polymer (Eq. (6.17)):

$q=\left\{\begin{array}{ll}q(0)=0.865 q_{R}^{0.88} & \text { fix } \\ q(0)\left[1+3.95 y^{1.54}\right]^{-0.44} & \text { var }\end{array}\right.$.

We find the internal concentrations $\phi$ from $\phi=\alpha \varphi$ or $\tilde{\phi}=\tilde{\alpha} \tilde{\varphi}$, with $\tilde{\phi}=\phi / \phi^{*}, \tilde{\varphi}=\varphi / \varphi^{*}$, and $\tilde{\alpha}=\alpha / \alpha^{*}$. For the critical points we use

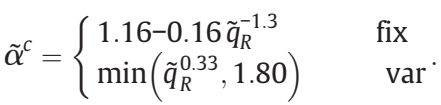

Eq. (7.6b) is the same as Eq. (6.40), but Eq. (7.6a) was not given before. It represents a simple power law from $\tilde{\alpha}^{c}=1$ in the cep to $\tilde{\alpha}^{c}=1.16$ for high $q_{R}$ (Fig. 5.11), and is nearly as accurate as the more complicated expression found by substituting $\eta^{c}=f^{c} /\left(1-f^{c}\right)$ with $f^{c}$ from Eq. (3.18) into Eq. (5.21).

For $\alpha^{t}$ in the triple point we need in the present chapter only the $\alpha$ 's in the fluid phases. We use a 'parabolic power law' not only for 'var' (Eqs. (6.42) and (6.44)) but also for 'fix':

$\tilde{\alpha}_{g}^{t}= \begin{cases}1+2.15 \sqrt{1-\tilde{q}_{R}^{-4}} & \text { fix } \\ 1+1.63 \sqrt{1-\tilde{q}_{R}^{-3}} & \text { var }\end{cases}$

$\tilde{\alpha}_{l}^{t}=\left\{\begin{array}{ll}1-\sqrt{1-\tilde{q}_{R}^{-5}} & \text { fix } \\ 1-0.69 \sqrt{1-\tilde{q}_{R}^{-4}} & \text { var }\end{array}\right.$.

The equations for the 'fixed' $\alpha$ 's in this section are less accurate than those given in chapter 5 (and 3). The most important difference is that the previous equations fully accounted for the exclusion limit, where for high $q$ the condensed phases are nearly (but not fully) void of polymer. Eq. (7.8) does not capture this exclusion limit quantitatively so when $\tilde{\alpha}_{l}^{t}$ is small the relative error may be large. However, for small $\alpha$ and $\phi$ accurate measurements of $\phi$ are very difficult and the absolute error in Eq. (7.8) is small anyhow. We expect that the simple Eqs. (7.2)-(7.8) are useful in interpreting the trends found in most experimental situations.

\subsection{The contact potential}

The phase behavior of colloids and atomic fluids is often described in terms of two parameters determining the pair potential between two colloids or atoms: the range and the strength of the interaction [24,62-66]. For example, a very popular system in which the range and strength are easily varied is a Yukawa fluid, consisting of hard spheres with an exponential attraction [64,67-69]. For such a fluid the pair potential $W(r)$ is infinite when the separation $r$ between the particle centres is smaller than $2 a$. In terms of the separation $H=r-2 a$ between the particle surfaces $W(H)=\infty$ when $H<0$. For $H>0$ the pair potential is given by

$W(H)=-\frac{\varepsilon}{1+H / 2 a} e^{-\kappa H} \quad W(h)=-\frac{\varepsilon}{1+h / 2} e^{-k h}$.

Here $\kappa^{-1}$ is the range of the interaction and $\varepsilon$ is the strength; it is (minus) the contact potential $W(0)$. In the second version of Eq. (7.9) we use the relative separation $h=H / a$ and the relative range $k^{-1}=\kappa^{-1} / a$.

For a colloid-polymer system the range is the depletion thickness $\delta$ and the relative range is the parameter $q=\delta / a$; we have discussed these parameters extensively in the preceding chapters. We now turn to the strength $\varepsilon$. When $\delta$ and $q$ are independent of the polymer concentration (or the osmotic pressure), a good approximation is $\varepsilon=\Pi v_{\mathrm{ov}}[20,70]$, where $v_{\mathrm{ov}}$ is the overlap volume of the two depletion layers for particles in contact. It is the overlap volume of two spherical shells with inner radius $a$ and outer radius $a+\delta: v_{\mathrm{ov}}=2 \pi \delta^{2} a(1+2 \delta / 3 a)$ $[20,70]$. For the relative overlap volume $v_{\text {ov }} / v$ we have then

$v_{\mathrm{ov}} / v=q^{2}(q+3 / 2)$.

Hence, we can write $\varepsilon$ also as $\varepsilon=\left(v_{\mathrm{ov}} / v\right) \Pi v$. Note the analogy of Eq. (7.10) with the coefficient $B$ in the free volume theory (Eq. (2.7)): $B=3 v_{\mathrm{ov}} / v$.

When $q$ does depend on $\varphi$ an integration procedure (build-up principle) is necessary, analogous to that used in the derivation of the polymer contribution to the grand potential (Eqs. (2.11) and (2.13)). The starting point is Gibbs' law $d \sigma=-\Gamma d \mu_{p}$, where $\sigma$ is the surface tension (from which $\varepsilon=W(0)$ follows) and $\Gamma$ is the (negative) adsorption of polymer, which is directly related to $\varphi v_{\mathrm{ov}}$. Applying 
the Gibbs-Duhem rule (analogous to the derivation of Eq. (2.20)) gives $\varphi \mathrm{d} \mu_{p}=(1-\varphi) d \Pi \approx d \Pi$. Integration of Gibbs' law ultimately leads to $[70,71]$

$\varepsilon=\int_{0}^{\Pi} v_{\mathrm{ov}} d \Pi=\int_{0}^{\Pi v}\left(v_{\mathrm{ov}} / v\right) d \Pi v=\int_{0}^{y}\left(v_{\mathrm{ov}} / v\right)(d \Pi v / d y) d y$

Clearly, for 'fix' this equation reduces to $\varepsilon=\Pi \mathrm{v}_{\mathrm{ov}}$. For 'var' the factor $v_{\mathrm{ov}} / v$ decreases with increasing $y$ (Eq. (7.10) with $q=q(y)$ ), and the factor $d \Pi v / d y$ increases (Eq. (6.12)). It turns out that these two opposing trends nearly cancel each other, and the integrand in the last form of Eq. (7.11) is approximately independent of $y$. Hence, a reasonable approximation is:

$\varepsilon=\frac{v_{\mathrm{ov}}}{v} y \frac{d \Pi v}{d y}=q^{2}\left(q+\frac{3}{2}\right) q_{R}^{-3} y\left(1+3.77 y^{1.31}\right)$

where $q=q\left(q_{R}, y\right)$ according to Eq. (7.5b). Comparison with the numerical integration of Eq. (7.11c) shows that Eq. (7.12) is very precise for $y<2$ and gives a slight overestimation, by less than about $20 \%$, for large $y$. In the dilute limit $y d \Pi v / d y$ equals $\Pi v$, in the semidilute limit $y d \Pi v / d y$ is larger than $\Pi v$ by a factor $3 \gamma=2.31$ (Eq. (6.12)), so $\varepsilon$ in Eq. (7.12) is larger than the simple form $\varepsilon=\Pi v_{\text {ov }}$ by this factor. The difference is due to the fact that $v_{\mathrm{ov}}$ decreases with increasing $\Pi$. When $v_{\mathrm{ov}}$ in $\int v_{\mathrm{ov}} d \Pi$ would be taken constant at its final value the integral would give $\Pi v_{\mathrm{ov}}$, but since over the integration interval $v_{\text {ov }}$ is larger than this final value the outcome of the integral is larger than $\Pi v_{\mathrm{ov}}$.

\subsection{Analytical results for the liquid window}

\subsubsection{The liquid window in terms of the interaction strength $\varepsilon$}

The interaction strength is given by Eq. (7.12) with Eq. (7.5) for $q$; for 'fix' we omit the term $y^{1.31}$ in Eq. (7.12). The values $\varepsilon^{c}(\mathrm{cp})$ and $\varepsilon^{t}$ (tp) are found by substituting Eq. (7.2) for $y^{c}=y^{*} \tilde{y}^{c}$ or Eq. (7.3) for $y^{t}=y^{*} \tilde{y}^{t}$. The result $\varepsilon^{c}\left(\tilde{q}_{R}\right)$ and $\varepsilon^{t}\left(\tilde{q}_{R}\right)$ is shown in Fig. 7.1: dashed curves for 'fix', solid curves for 'var'. Fig. 7.2 shows the same data in the representation $\varepsilon^{c}(\tilde{q})$ and $\varepsilon^{t}(\tilde{q})$. In addition, we show in Fig. 7.2 results for a Yukawa fluid, as presented recently [64] in an analytical approximation for this system. In this case the parameter $q_{R}$ has no meaning, but we can plot $\varepsilon^{c}$ and $\varepsilon^{t}$ as a function of the normalized range $1 / \tilde{k}=k^{*} / k$, which is equivalent to $\tilde{q}=q / q^{*}$ for a colloid/polymer system.

We first compare the cep in the three models in Fig. 7.2. Perhaps the most important conclusion is that the cep is situated at approximately the same value $\varepsilon^{*}=2.1$ ( $k T$ units) in the three cases ('fix', 'var', and 'Yuk'). The same value follows from Monte Carlo simulations [72] based upon a Lennard-Jones-type potential. Not only the strength $\varepsilon^{*}$ in the cep is the same in these four systems, also the

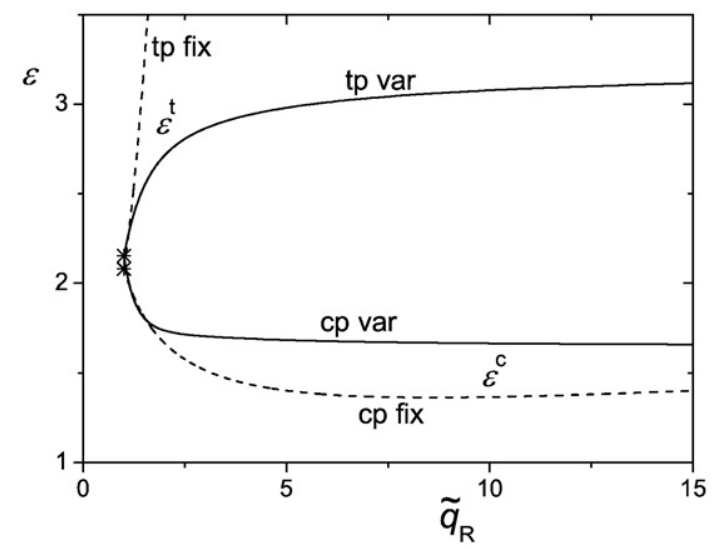

Fig. 7.1. The dependence of $\varepsilon^{c}$ (cp) and $\varepsilon^{t}$ (tp) on $\tilde{q}_{R}=q_{R} / q_{R}^{*}$, for both 'fix' (dashed curves) and 'var' (solid curves), according to Eqs. (7.2), (7.3) and (7.12). For 'fix' the factor 3.77 in Eq. (7.12) is taken zero.

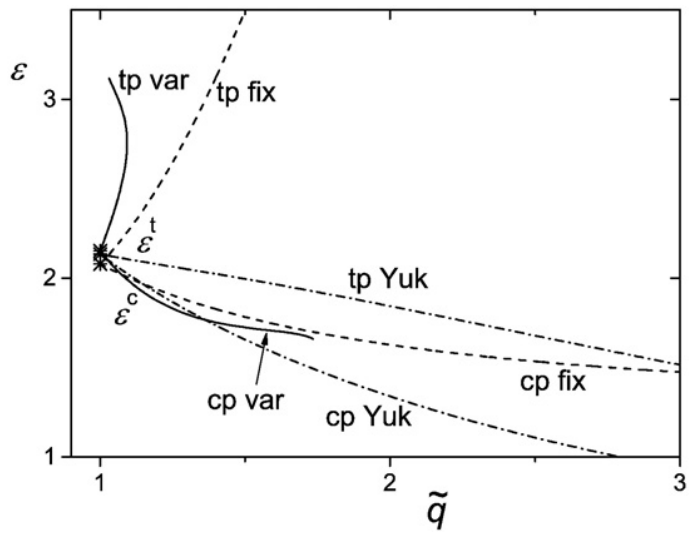

Fig. 7.2. The dependence of $\varepsilon^{c}$ (cp) and $\varepsilon^{t}$ (tp) on $\tilde{q}=q / q^{*}$, for 'fix' (dashed curves), 'var' (solid curves), and 'Yuk' (dash-dotted). The latter data are taken from [64]. The abbreviation Yuk stands for a Yukawa fluid, where $1 / \tilde{k}=k^{*} / k$ plays the same role as $\tilde{q}$.

relative range $q^{*}$ (or $1 / k^{*}$ in the Yukawa model) is roughly the same, around 0.3. This suggests that for a collection of particles with a smooth attractive pair potential a universal principle applies: liquid is only possible if the range is longer than roughly one third of the particle radius. In addition, for a Yukawa system the strength of the interaction should be smaller than about $2 \mathrm{kT}$ : for stronger interactions and/or a smaller range than one-third of the particle radius the solid state is the preferred situation for the condensed phase.

Outside the cep such a universality does not apply and the liquid window depends strongly on the type of interaction. We first discuss the $\varepsilon\left(\tilde{q}_{R}\right)$ plots for 'fix' and 'var' in Fig. 7.1, and then turn again to Fig. 7.2 where also the Yukawa results are shown.

For 'fix' in Fig. 7.1 the liquid window is wide. The value $\varepsilon^{c}$ for the critical point decreases from 2.1 in the cep to roughly 1.4 around $q_{R}=8 q_{R}^{*}$, and for higher $q_{R}$ it increases again weakly. This behavior follows from Eq. (7.12) in the form $\varepsilon \sim q^{2} q_{R}^{-3} y$ (low $q$ ) or $\varepsilon \sim q^{3} q_{R}^{-3} y$ (high $q$ ), which with $q \sim q_{R}^{0.88}$ and $y^{c} \sim q_{R}^{0.72}$ (Eq. (7.2a)) leads to $\varepsilon^{c} \sim q_{R}^{-0.5}$ for low $q$ and $\varepsilon^{c} \sim q_{R}^{0.4}$ for (very) high $q$, so there must be an intermediate (weak) minimum. For $\varepsilon^{t}$ in 'fix' we have to substitute $y^{t} \sim q_{R}{ }^{3}$, which leads to a very strong increase of $\varepsilon^{t}$ with $q_{R}: \varepsilon^{t} \sim q^{2} \sim q_{R}^{1.86}$ for low $q$, and $\varepsilon^{t} \sim q^{3} \sim q_{R}^{2.64}$ for high $q$. Hence, in this (irrealistic) case the liquid window in terms of $\varepsilon^{t}-\varepsilon^{c}$ is wide, increasing without bounds.

For 'var' in Fig. 7.1 the behavior very close to the cep is similar to that for 'fix'; here we are still (more or less) in the dilute regime. However, for high $q_{R}$ both $y^{t}$ and $y^{c}$ exceed unity and we enter the semidilute regime where the $q_{R}$-dependence disappears. The final levels are found by substituting the high- $q_{R}$ limit $y=A y^{*} \tilde{q}_{R}^{1.3}$ into Eq. (7.12), where we neglect the unity term in the last factor. The result is $\varepsilon=8.96 q^{2}(q+1.5) A^{2.31}$. For the critical point $q^{c}=0.48$ (Eq. (6.30)) and $A=0.66$ (Eq. (7.2)), which gives a final level $\varepsilon^{c}=1.64$; for the triple point we substitute $q^{t}=0.29$ (Eq. (6.30)) and $A=1.42$ (Eq. (7.3)) to find $\varepsilon^{t}=3.06$. Hence, the liquid window for high $q_{R}$ has a width $\varepsilon^{t}-\varepsilon^{c}=1.4$ ( $k T$ units).

The behavior of a Yukawa system is quite different. Here it makes no sense to make a plot $\varepsilon\left(\tilde{q}_{R}\right)$; instead we consider the plot $\varepsilon(\tilde{q})$ in Fig. 7.2, which for a Yukawa system is equivalent to a plot $\varepsilon(1 / \tilde{k})$. The 'fixed $q$ ' curves for $\varepsilon^{t}$ and $\varepsilon^{c}$ in Fig. 7.2 are qualitatively similar to those in Fig. 7.1 because $q$ is of order $q_{R}\left(\tilde{q}=\tilde{q}_{R}^{0.88}\right)$. Note that the abscissa scales are different in the two figures: the highest $\varepsilon^{t}(=3.5)$ is reached at $\tilde{q}=1.6$ in Fig. 7.2, which corresponds to $\tilde{q}_{R}=1.56$ in Fig. 7.1. Again we see a wide liquid window for 'fix'. The Yukawa plot is qualitatively different: both $\varepsilon^{t}$ and $\varepsilon^{c}$ decrease when the interaction range increases. In a rough approximation this decrease can be described as simple power laws $\tilde{\varepsilon}^{t}=\tilde{k}^{0.3}=\tilde{q}^{-0.3}$ and $\tilde{\varepsilon}^{c}=\tilde{k}^{0.7}=\tilde{q}^{-0.7}$. Moreover, it can be seen that the liquid window is quite narrow: even for the highest $\tilde{q}=1 / \tilde{k}$ indicated in the figure $\varepsilon^{t}-\varepsilon^{c}$ is no more than 0.6 ( $k T$ units). This illustrates the subtle balance for the existence of a 
liquid phase in a Yukawa system, where the attractions are pair-wise additive only.

The 'var' data in Fig. 7.2 span only a narrow range of $\tilde{q}$. The value of $q^{t}$ hardly varies with $q_{R}$ (see Fig. 6.5) so $\varepsilon^{t}$ increases from 2.1 to 3.1 with $\tilde{q}^{t}$ quite close to unity over the whole range of $q_{R}$. The curve for $\varepsilon^{c}$ more or less coincides with that for 'fix' (and 'Yuk') but $\tilde{q}^{c}$ varies only from 1 to roughly 1.75 and then stops.

\subsubsection{The liquid window in terms of the external polymer concentration $\varphi$}

The external polymer concentration in triple and critical points is given by Eq. (7.4) in combination with Eqs. (7.2) and (7.3) for $y^{c}$ and $y^{t}$. Fig. 7.3 gives the result for a fixed polymer radius $R$. Then $q_{R}$ can only be changed by varying the particle radius $a$. The parameter $\varphi_{\text {ov }}$ is now independent of $q_{R}$ so that $\tilde{\varphi}^{c}=\tilde{y}^{c}$ and $\tilde{\varphi}^{t}=\tilde{y}^{t}$ (Eq. (7.4a)). Fig. 7.3 shows the normalized quantities $\tilde{\varphi}^{c}$ and $\tilde{\varphi}^{t}$, which are directly given by Eq. (7.2)

For 'fix' there is a moderate increase of $\varphi^{c}$ with $q_{R}$ according to the simple power law $\tilde{\varphi}^{c}=\tilde{q}_{R}^{0.72}$. For the triple point we have a much stronger dependence according to a double power law $\tilde{\varphi}^{t}=\tilde{q}_{R}^{1.4}$ close to the cep to $\tilde{\varphi}^{t}=0.57 \tilde{q}_{R}{ }^{3}$ at high $q_{R}$. Consequently, the liquid window is again wide for 'fix', with for high $q_{R} \varphi^{t} / \varphi^{c}$ diverging as $q_{R}^{2.3}$.

For 'var' this window is narrower. Now we have a double power law for both $\varphi^{c}$ and $\varphi^{t}$. Very close to the cep $\tilde{\varphi}^{c}=\tilde{q}_{R}^{0.72}$ (Eq. (6.34)), which is the same as for 'fix'. In this region $\tilde{\varphi}^{t}=\tilde{q}_{R}^{1.55}$ for 'tp var', which is again not much different from 'tp fix' $\left(\tilde{q}_{R}^{1.4}\right)$. For most of the $q_{R}$ range in Fig. 7.3 the high- $q_{R}$ limit is more relevant. In this limit we have for 'cp var' $\tilde{\varphi}^{c}=0.66 \tilde{q}_{R}^{1.3}$ and for 'tp var' $\tilde{\varphi}^{c}=1.42 \tilde{q}_{R}^{1.3}$ : both quantities increase with the same power of $q_{R}$. However, the ratio $\varphi^{t} / \varphi^{c}$, which is unity at the cep, quickly converges to a constant value $1.42 / 0.66=2.2$.

Fig. 7.4 gives the analogous results $\tilde{\varphi}\left(\tilde{q}_{R}\right)=\tilde{Y}\left(\tilde{q}_{R}\right)$ for a constant particle radius $a$. Now Eq. (7.4b) applies: the factor $\tilde{q}_{R}^{-1.3}$ originates from the fact that $\varphi_{\mathrm{ov}}$ varies with $q_{R}$. This additional factor $\tilde{q}_{R}^{-1.3}$ gives a completely different Fig. 7.4 as compared to Fig. 7.3. The divergence in 'tp fix' remains $\left(\varphi^{\mathrm{t}} \sim q_{R}^{3-1.3}=q_{R}^{1.7}\right)$ but 'cp fix' now decreases as $\varphi^{c} \sim q_{R}^{0.72-1.3}=q_{R}^{-0.6}$ for high $q_{R}$. For the 'var' curves we find a constant level for high $q_{R}$ because the prefactor $\tilde{q}_{R}^{1.3}$ in Eqs. (7.2) and (7.3) is cancelled by the factor $\tilde{q}_{R}^{-1.3}$ in Eq. (7.4). Despite the differences in the absolute values of $\tilde{\varphi}^{c}$ and $\tilde{\varphi}^{t}$ in Figs. 7.3 and 7.4 the ratios $\varphi^{t} / \varphi^{c}$ are the same in both figures: $\varphi^{t} / \varphi^{c} \sim q_{R}^{2.3}$ for 'fix', and $\varphi^{t} / \varphi^{c}=2.2$ for 'var'. The liquid window in 'var' spans a factor 2.2 in (external) polymer concentration.

\subsubsection{The liquid window in terms of the internal polymer concentration $\phi$}

Figs. 7.3 and 7.4 for the external concentrations $\tilde{\varphi}\left(\tilde{q}_{R}\right)$ may be converted to the internal concentrations $\tilde{\phi}\left(\tilde{q}_{R}\right)$ by multiplying $\tilde{\varphi}$ by $\tilde{\alpha}$,

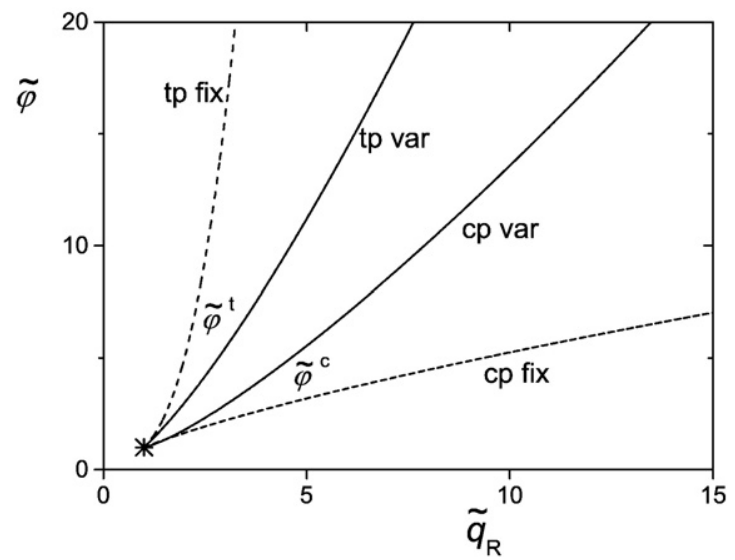

Fig. 7.3. The dependence of $\varphi^{c}(\mathrm{cp})$ and $\varphi^{t}(\mathrm{tp})$ (both normalized on $\varphi^{*}$ ) on $\tilde{q}_{R}=q_{R} / q_{R}^{*}$ for the case of constant $R$, for 'fix' (dashed curves) and 'var' (solid curves).

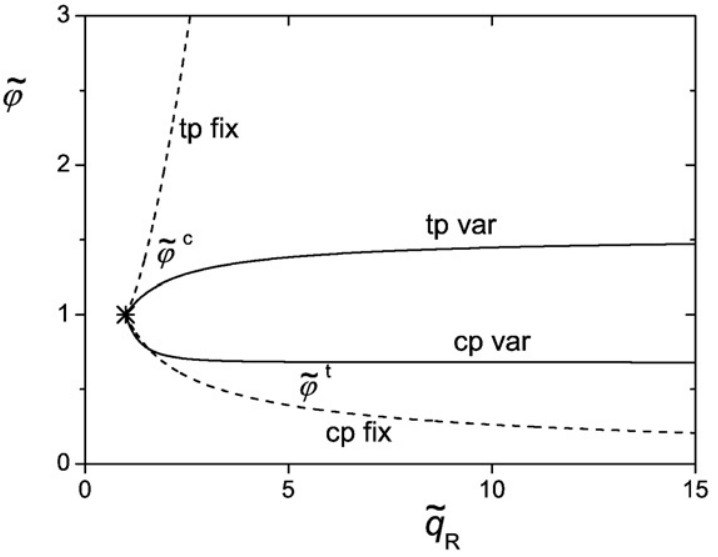

Fig. 7.4. The dependence of $\varphi^{c}(\mathrm{cp})$ and $\varphi^{t}(\mathrm{tp})$ (all normalized on $\varphi^{*}$ ) on $\tilde{q}_{R}=q_{R} / q_{R}^{*}$ for the case of constant $a$, for 'fix' (dashed curves) and 'var' (solid curves).

given by Eq. (7.6) (cp), (7.7) (tpG), and (7.8) (tpL). Fig. 7.5 gives the analogue of Fig. 7.3 (constant R), Fig. 7.6 is analogous to Fig. 7.4 (constant $a$ ). For $\phi^{t}$ we have now two branches tpG (gas) and tpL (liquid).

For 'fix' the critical curve for $\phi^{c}$ in Fig. 7.5 is about the same as that for $\varphi^{c}$ in Fig. $7.3\left(\phi^{c} \sim q_{R}{ }^{0.72}\right)$. This is because $\tilde{\alpha}^{c}$ is close to unity for any $q_{R}$ : it varies from $\tilde{\alpha}^{c}=1$ at the cep to 1.16 at high $q_{R}$ (Eq. (7.6a)). Also the tpG curve is qualitatively the same: here $\tilde{\alpha}_{g}^{t}$ increases from $\tilde{\alpha}^{c}=1$ at the cep to $\tilde{\alpha}_{g}^{t}=1 / \alpha^{*}=3.2$ at high $q_{R}$. The tpL curve is quite different because $\tilde{\alpha}_{l}^{t}$ decays from 1 at the cep to zero for high $q_{R}$.

The 'var' curves in Fig. 7.5 differ considerably from those for 'fix'. The basic point is that all the $\alpha$ 's reach a final $q_{R}$-independent value in the protein limit: $\tilde{\alpha}^{c}=1.8, \tilde{\alpha}_{g}^{t}=1 / \alpha^{*}=2.6$, and $\tilde{\alpha}_{l}^{t}=0.31$. As a consequence, the scaling behavior of $\phi$ (internal) for high $q_{R}$ is the same as that of $\varphi$ (external): $\tilde{\phi}^{c}=1.19 \tilde{q}_{R}^{1.3}, \tilde{\phi}_{g}^{t}=3.74 \tilde{q}_{R}^{1.3}$, and $\tilde{\phi}_{l}^{t}=0.44 \tilde{q}_{R}^{1.3}$. The ratios $\phi_{g}^{t} / \phi^{c}$ and $\phi_{l}^{t} / \phi^{c}$ are then again constant: 3.14 and 0.37 , respectively.

Fig. 7.6 displays the analogous data for $\tilde{\phi}$ in critical and triple points for the case of constant $a$. Basically, this figure is obtained by multiplying $\tilde{\phi}$ in Fig. 7.5 by $\tilde{q}_{R}^{-1.3}$ Eq. (7.4). The result for 'fix' is a weaker (but still strong) increase of $\phi_{g}^{t}$ with $q_{R}$, and a faster decay to zero of $\phi_{l}^{t}$. For the 'cp fix' curve the increase $\left(\phi^{c} \sim q_{R}^{0.72}\right)$ in Fig. 7.5 is converted into a decrease $\left(\phi^{c} \sim q_{R}^{-0.58}\right)$ in Fig. 7.6. For 'cp var' and 'tp var' the high- $q_{R}$ increase $\phi \sim q_{R}^{1.3}$ in Fig. 7.5 is just compensated by the $q_{R}^{-1.3}$ dependence of Eq. (7.4), so $\phi^{c}$ and $\phi^{t}$ reach a constant level in Fig. 7.6. Nevertheless, the same ratios $\phi_{g}^{t} / \phi^{c}=3.1$ and $\phi_{l}^{t} / \phi^{c}=0.37$ apply as in Fig. 7.5. The liquid window in terms of $\phi_{g}^{t} / \phi_{l}^{t}$ is a factor of about 8 in internal concentrations.

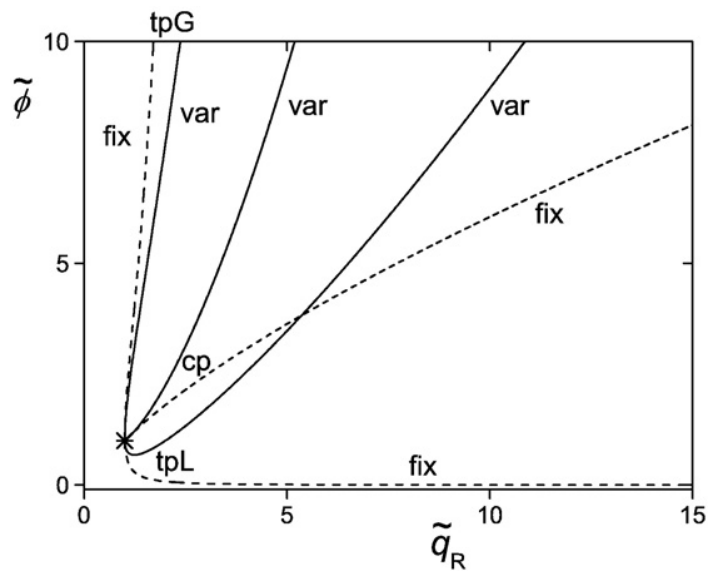

Fig. 7.5. The dependence of $\phi^{c}(\mathrm{cp}), \phi_{g}^{t}(\mathrm{tpG})$, and $\phi_{l}^{t}(\mathrm{tpL})$ (all normalized on $\varphi^{*}$ ) on $\tilde{q}$ ${ }_{R}=q_{R} / q_{R}^{*}$ for the case of constant $R$, for 'fix' (dashed curves) and 'var' (solid curves). 


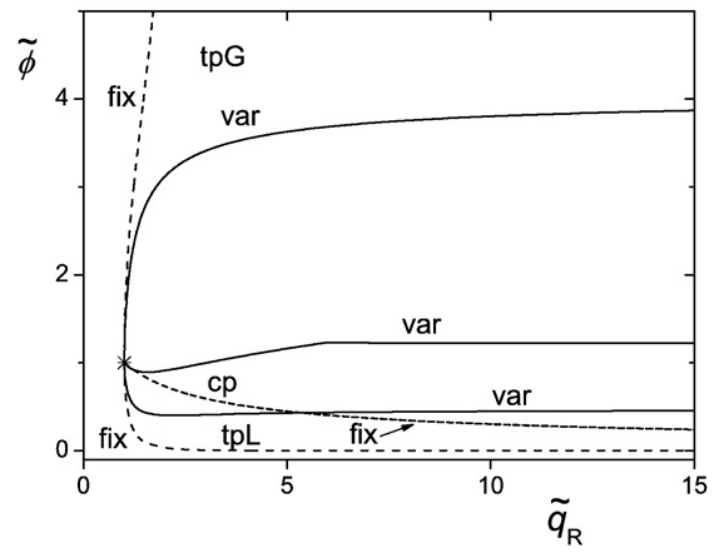

Fig. 7.6. The dependence of $\phi^{c}(\mathrm{cp}), \phi_{g}^{t}(\mathrm{tpG})$, and $\phi_{l}^{t}(\mathrm{tpL})$ (all normalized on $\left.\varphi^{*}\right)$ on $\tilde{q}$ ${ }_{R}=q_{R} / q_{R}^{*}$ for the case of constant $a$, for 'fix' (dashed curves) and 'var' (solid curves).

All the results presented in this chapter are for strictly monodisperse colloidal particles. In experimental systems unavoidably some polydispersity occurs, which impedes crystallization. This affects the position of the cep (where a critical fluid is in equilibrium with a crystalline phase). Far from the cep, where GL demixing is stable with respect to FS demixing the effect of polydispersity is probably much less. In the next chapter we will return to this issue of polydispersity.

\section{Comparison with experiments and simulations}

In this chapter we compare the analytical predictions of our 'variable $q$ ' theory as discussed in chapter 6 with experiments, with computer simulations, and with other theories. Since in literature studies the polymer concentrations $\phi$ are commonly normalized by the overlap concentration $\varphi_{\mathrm{ov}}$, we introduce the quantity $y_{i}=\phi / \varphi_{\mathrm{ov}}=$ $\alpha y$ for the internal polymer concentration relative to overlap.

Experiments usually provide (part of) GL binodals. In Section 8.1 we start therefore with a few examples of GL binodals, for both good and theta solvents. A central feature is the critical point (cp), for which our theory provides very simple analytical expressions. From experiments it is sometimes possible to estimate the cp, but in many cases this point is not accurately known. Then we employ a rough procedure to extract an approximate cp value from the experimental binodal. Results are discussed and compared with theory in Section 8.2; this section contains a table with all the information on cp's from experiment which we could find in the literature.

Sections 8.3 (GL binodals) and 8.4 (cp's) gives an analogous comparison with simulations and other theoretical models. In this case we could also check to what extent these other models follow the scaling law for the protein limit $\left(\phi \sim q_{R}^{1 / \gamma}\right)$ as derived in chapter 6 . In Section 8.5 we compare the free volume fraction $\alpha$ from simulations with the scaled-particle expression (Eqs. (2.5)-(2.7)) for 'variable $q$ '.

In Section 8.6 we discuss an experimental example where the position of the tie-lines is measured explicitly. In Section 8.7 we interpret the (very scarce) experimental information about triple points. Finally, in Section 8.8 we discuss a complete experimental $y_{i}(\eta)$ phase diagram for $q_{R}=1$ (i.e., colloids and polymer have the same size). In this study all twophase regions GL, FS, and GS plus the three-phase GLS-region (inside the triple triangle) were observed. We find that our new 'var' theory describes this experimental phase diagram excellently.

\subsection{GL binodals from experiment}

Fig. 8.1 gives four experimental binodals $y_{i}(\eta)$ for good-solvent conditions. The circles $\left(q_{R}=1.40\right)$, squares $\left(q_{R}=0.67\right)$, and plusses $\left(q_{R}=0.38\right)$ are for silica particles (coated with octadecanol) of radius $a=50 \mathrm{~nm}$ in toluene in the presence of polystyrene (PS) of different molar masses [73], the diamonds are for a polysaccharide-casein micelle mixture $\left(q_{R}=0.86\right)$ in water [74]. The curves in Fig. 8.1 are our analytical binodals. We stress that there are no adjustable parameters; the only input is $q_{R}$. The three closed circles on each of these curves are, from left to right, the gas side tpG of the triple point tp, the critical point $\mathrm{cp}$, and the liquid side tpL of tp, calculated from the analytical ev equations presented in Section 6.4. The full binodal was found from tpG, cp, and tpL using the analytical equations for $y=\varphi / \varphi_{\text {ov }}$ and $y_{i}=\alpha y$ as a function of $\eta$ as given in Section 6.8.

For the two middle curves in Fig. $8.1\left(q_{R}=0.67,0.86\right)$ the agreement between theory and experiment is nearly quantitative. Earlier attempts $[30,71]$ to interpret these experimental binodals with existing theories were less successful. This constitutes an indication that our new theory is viable. However, for $q_{R}=1.4$ we seem to overestimate the experimental binodal. Possibly, the reason is the polydispersity in this experimental system.

For $q_{R}=0.38$ our model does not give stable GL demixing, as this value is below the theoretical cep at $q_{R}^{*}=0.388$. Again we have to realize that the colloid particles in the experiment are unavoidably somewhat polydisperse, whereas the theory is for strictly monodisperse particles which crystallize easier than polydisperse ones. Kofke and Bolhuis [75] have shown that a very polydisperse hardsphere dispersion does not crystallize at all. Since the cep is determined by equilibrium with a crystalline phase, for moderate polydispersity it is quite possible that $q_{R}^{*}$ is lower than 0.388 , so that a liquid of polydisperse particles is stable at $q_{R}=0.38$. It is difficult to quantify this polydispersity effect theoretically; some attempts have been made by Sollich and Fasolo [76,77]. When we model the polydisperse system by a 'monodisperse' theory by rescaling $q_{R}$ around the cep slightly, we might find reasonable results. The experimental data points for $q_{R}(\exp )=0.38$ are well described by a theoretical binodal for $q_{R}($ th $)=0.44$, as seen in Fig. 8.1. We return to this rescaling of $q_{R}$ around the cep due to polydispersity in Section 8.7.

Fig. 8.2 gives similar data for a theta solvent. The circles $\left(q_{R}=0.84\right)$ are for the same silica/PS system [73] as in Fig. 8.1, but now the solvent is cyclohexane, a theta solvent for PS. The diamonds $\left(q_{R}=1.4\right)$ and squares $\left(q_{R}=2.2\right)$ are for an AOT/water/cyclohexane oil-continuous microemulsion ( $\mu \mathrm{em}$ ) with added PS [78] (cyclohexane is again a theta solvent). The curves in Fig. 8.2 are theoretical binodals, where now the $\mathrm{mf}$ version of the equations was used.

The overall agreement between theory and experiment is quite reasonable. For the silica/PS system the experimental binodal is somewhat flatter than the theoretical one, whereas those for $\mu \mathrm{em} / \mathrm{PS}$ seem to be steeper. These differences might be due to colloid and/or

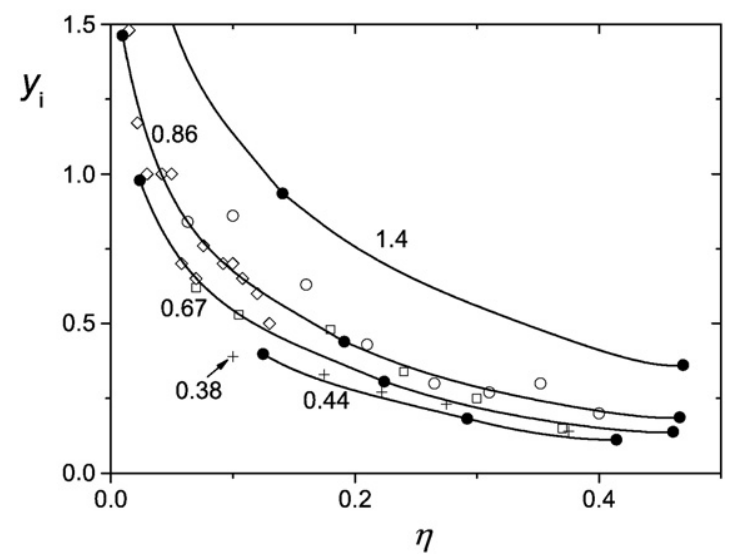

Fig. 8.1. Comparison of experimental good-solvent GL binodals for $q_{R}=0.38$ (plusses, [7.3]), 0.67 (squares, [7.3]), 0.86 (diamonds, [74]), and 1.4 (circles, [73]) with theoretical binodals for the same $q_{R}$ values (except for the bottom curve, which was computed with $\left.q_{R}=0.44\right)$. The closed circles on the theoretical binodals correspond, from left to right, with tpG, cp, and tpL. 


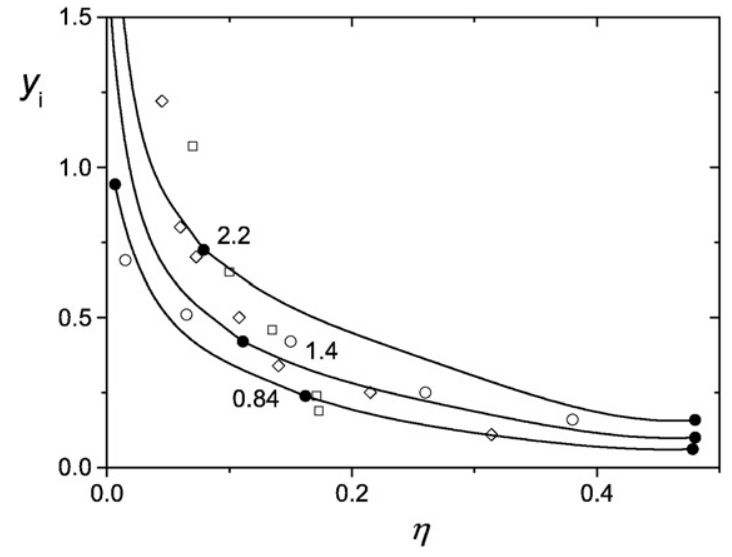

Fig. 8.2. Comparison of experimental theta-solvent GL binodals for $q_{R}=0.84$ (circles, [73]), 1.4 (diamonds, [78]), and 2.2 (squares, [78]) with theoretical binodals for the same $q_{R}$ values. The closed circles on the theoretical binodals correspond, from left to right, with tpG, cp, and tpL.

polymer polydispersity effects. Increasing polymer polydispersity leads to steeper tie-lines [77], whereas more polydisperse colloids make the tie-lines flatter [76]. Overall our 'monodisperse' theory still describes the trends sufficiently well.

In the experimental data of Figs. 8.1 and 8.2 no direct information on the $\mathrm{cp}$ is available. For comparing the various experimental binodals available in the literature, it would be convenient to have an indication for the position of the $\mathrm{cp}$. We obtained this by taking the theoretical value of $\eta^{c}$ and estimating what value of $y_{i}{ }^{c}$ corresponds to it according to the experimental binodal. For example, for $q_{R}=0.84$ in Fig. 8.2 the theoretical value (indicated by the closed circle) is $y_{i}^{c}=0.26$ at $\eta^{c}=0.16$. The experimental data points (diamonds) lie somewhat above the theoretical binodal and suggest $y_{i}^{c} \approx 0.4$ at $\eta^{c}=0.16$. We take this $y_{i}$ value as an estimate for the experimental $y_{i}^{c}$. Data of this type are collected in Table 8.1 (next section) whenever no 'true' experi- mental cp is reported; they may not provide the real cp but do give an indication about how well the theoretical binodal fits the experimental one.

\subsection{Critical points from experiment}

Table 8.1 gives an overview of experimental cp data. Whenever an experimental value $\eta^{c}, y_{i}^{c}$ was reported, this $\eta^{c}$ is indicated in the fourth column. When we estimated $y_{i}^{c}$ from the experimental binodal as indicated at the end of Section 8.1, the 'experimental' $\eta^{c}$ value is left blank in this column.

The last four columns give our analytical predictions for the $q_{R}$ value as reported in column 3 . Here we have to make a choice whether to use the ev version for a good solvent or the mf version for a theta solvent. We subdivided the table in three parts. On top we collected those systems where it is reasonable to assume good-solvent conditions, and at the bottom we have the theta-solvent data. The middle part refers to borderline cases, where the solvent is marginally better than theta, with $\chi$-values around 0.48 [84]. Here we indicated both the ev predictions and those for mf, expecting that these two cases would represent upper and lower limits, respectively, for the actual system.

Our theory predicts that $\eta^{c}$ decreases with increasing $q_{R}$ and reaches a constant level ( $0.11 \mathrm{for} e v, 0.06$ for $\mathrm{mf}$ ) in the protein limit. There is not much direct experimental evidence to support these predictions. However, the few data in column four are rather close to those in column six, and a high- $q_{R}$ level $\eta^{c} \approx 0.1$ seems to be corroborated.

In contrast, $y_{i}^{c}$ is predicted to increase with $q_{R}$, and for high $q_{R}$ the scaling law $y_{i}^{c} \sim q_{R}^{1 / \gamma}$ (hence, $y_{i}^{c} \sim q_{R}$ in a theta solvent and $y_{i}^{c} \sim q_{R}^{1.3}$ in a good solvent) should apply. The general trend in column five is indeed an increase with $q_{R}$, although the two silica/PDMS/cyclohexane data look rather high. Probably, this is caused by the fact that PDMS weakly attaches to stearylated silica in cyclohexane [86], which decreases the depletion thickness as compared to fully repulsive interactions. Therefore higher PDMS concentrations are required to

Table 8.1

Comparison of experimental and theoretical critical points

\begin{tabular}{|c|c|c|c|c|c|c|c|c|}
\hline \multirow[t]{2}{*}{ Ref } & \multirow[t]{2}{*}{ System } & \multirow[t]{2}{*}{$q_{R}$} & $\underline{\eta^{c}}$ & \multirow[t]{2}{*}{$y_{i}^{c}$} & \multirow[t]{2}{*}{$\eta^{c}$} & \multirow[t]{2}{*}{$y_{i}^{c}$} & \multirow[t]{2}{*}{$\eta^{c}$} & \multirow[t]{2}{*}{$y_{i}^{c}$} \\
\hline & & & $\exp$ & & & & & \\
\hline \multicolumn{9}{|l|}{ ev } \\
\hline [79] & sil/PDMS/chx & 0.49 & 0.21 & 1.00 & 0.27 & 0.21 & & \\
\hline [73] & sil/PS/tol & 0.67 & - & 0.35 & 0.22 & 0.30 & & \\
\hline [74] & cas/psc/w & 0.86 & - & 0.45 & 0.19 & 0.41 & & \\
\hline [81] & $\gamma \mathrm{Sprot} / \mathrm{PEO} / \mathrm{w}$ & 0.87 & 0.18 & 0.34 & 0.19 & 0.42 & & \\
\hline [82] & sil/PDMS/chx & 1.08 & - & 1.6 & 0.17 & 0.58 & & \\
\hline [73] & sil/PS/tol & 1.40 & - & 0.65 & 0.14 & 0.87 & & \\
\hline [83] & $\mathrm{wp} / \mathrm{psc} / \mathrm{w}$ & 3.2 & - & 3.5 & 0.11 & 3.0 & & \\
\hline \multicolumn{9}{|l|}{$\mathrm{ev} / \mathrm{mf}$} \\
\hline [80] & PMMA/PS/dec & 0.56 & 0.1 & 0.36 & 0.25 & 0.24 & 0.22 & 0.18 \\
\hline [7] & PMMA/PS/dec & 0.57 & - & 0.25 & 0.25 & 0.25 & 0.22 & 0.19 \\
\hline$[84,85]$ & sil/PS/tol & 4.2 & 0.13 & 2.2 & 0.11 & 4.2 & 0.06 & 1.52 \\
\hline$[84,85]$ & sil/PiP/chx & 4.8 & 0.13 & 1.6 & 0.11 & 5.0 & 0.06 & 1.74 \\
\hline$[84,85]$ & sil/PS/tol & 5.2 & 0.09 & 2.6 & 0.11 & 5.6 & 0.06 & 1.89 \\
\hline \multicolumn{9}{|l|}{$\mathrm{mf}$} \\
\hline [73] & sil/PS/chx & 0.84 & - & 0.40 & & & 0.16 & 0.26 \\
\hline [78] & $\mu \mathrm{em} / \mathrm{PS} / \mathrm{chx}$ & 1.3 & - & 0.35 & & & 0.12 & 0.39 \\
\hline [78] & $\mu \mathrm{em} / \mathrm{PS} / \mathrm{chx}$ & 1.4 & - & 0.55 & & & 0.11 & 0.43 \\
\hline [78] & $\mu \mathrm{em} / \mathrm{PS} / \mathrm{chx}$ & 2.1 & - & 0.60 & & & 0.08 & 0.68 \\
\hline [78] & $\mu \mathrm{em} / \mathrm{PS} / \mathrm{chx}$ & 2.2 & - & 0.85 & & & 0.08 & 0.72 \\
\hline [78] & $\mu \mathrm{em} / \mathrm{PS} / \mathrm{chx}$ & 7.5 & - & $<0.1$ & & & 0.06 & 2.7 \\
\hline [78] & $\mu \mathrm{em} / \mathrm{PS} / \mathrm{chx}$ & 8.9 & - & 0.3 & & & 0.06 & 3.2 \\
\hline
\end{tabular}

\section{Abbreviations:}

sil $=($ stearyl $)$ silica, PMMA $=$ polymethylacrylate, cas $=$ caseine micelles, $w p=$ whey proteins, $\gamma$ Sprot $=$ eye lens protein, $\mu \mathrm{em}=\mathrm{AOT} / \mathrm{water}$ microemulsion .

$\mathrm{PDMS}=$ polydimethyl siloxane, $\mathrm{PS}=$ polystyrene, $\mathrm{psc}=$ polysaccharide, $\mathrm{PEO}=$ polyethylene oxide, $\mathrm{PiP}=$ polyisoprene .

ch $\mathrm{x}=$ cyclohexane, tol $=$ toluene, dec $=$ decalin, $\mathrm{w}=$ water 
induce phase separation as compared to purely non-adsorbing polymer chains.

There are not enough data to experimentally verify the power law dependence of $y_{\mathrm{i}}^{c}$ on $q_{R}$. The results in the middle part of the table suggest that the $y_{i}^{c}$ data for $q_{R}=0.6$ are better described by the ev equations than by the $\mathrm{mf}$ version; for $q_{R}$ around 5 the $\mathrm{mf}$ description looks slightly better. In practice, the solvency may depend on polymer concentration $\phi$, which complicates the analysis.

The lower part of Table 8.1, referring to theta solvents, show that most of the data are well described by our theoretical model. The obvious exception is for the systems with $q_{R}$ around 8, where the experimental binodal lies at very low $y_{i}$, with cp values even lower than for $q_{R}=1-2$; the reason is unknown to us. Possibly, weak polymer adsorption affects depletion effects.

\subsection{GL binodals from simulations}

Bolhuis et al. [40,87] performed large-scale Monte Carlo computer simulations to obtain GL binodals for various size ratios $q_{R}$ in the excluded-volume limit. Their results [87] for $q_{R}=0.67$ (crosses) and $q_{R}=1.05$ (plusses) are given in Fig. 8.3, together with our theoretical predictions (curves). Unlike the simulations, our model gives also the precise coordinates of the $\mathrm{cp}$ (which can only be estimated from simulations) and of the tp (which is very difficult to obtain in simulations). The values of $\mathrm{tpG}, \mathrm{cp}$, and tpL are again indicated as the closed circles on the theoretical binodals.

For $q_{R}=0.67$ the theoretical binodal is very close to the simulations, although the simulation data give a slightly flatter curve at the gas side. For $q_{R}=1.05$ there is fair agreement but now the theoretical binodal overestimates the simulation data somewhat, especially at the extremes. Note that the two simulated binodals cross at the liquid side. We do not have any theoretical argument which would lead to such intersecting binodals.

Fig. 8.4 gives simulation data [40] for three high $q_{R}$ values 3.86 (crosses), 5.58 (plusses), and 7.78 (diamonds). As these values correspond to the protein limit, we did not plot $y_{i}$ itself, but $y_{i} q_{R}^{-1.3}$ according to the scaling picture, $y_{i}{ }^{c} \sim q_{R}^{1 / \gamma}$, of chapter 6 . It is gratifying to see that the three simulation binodals now collapse onto a single curve, which corroborates our scaling law. We note that this law does not depend on the specifics of a theoretical model (such as the numerical constants for $\mathrm{cp}$ and tp in our theory) so it should have general validity, as the only input is the De Gennes scaling exponent $\gamma$ for semidilute solutions.

The next point is the quantitative comparison with our theory. We predict $\eta_{\mathrm{g}}^{t}(\approx 0), \eta^{c}(\approx 0.11)$, and $\eta_{l}^{t}(\approx 0.48)$ to become independent of $q_{R}$

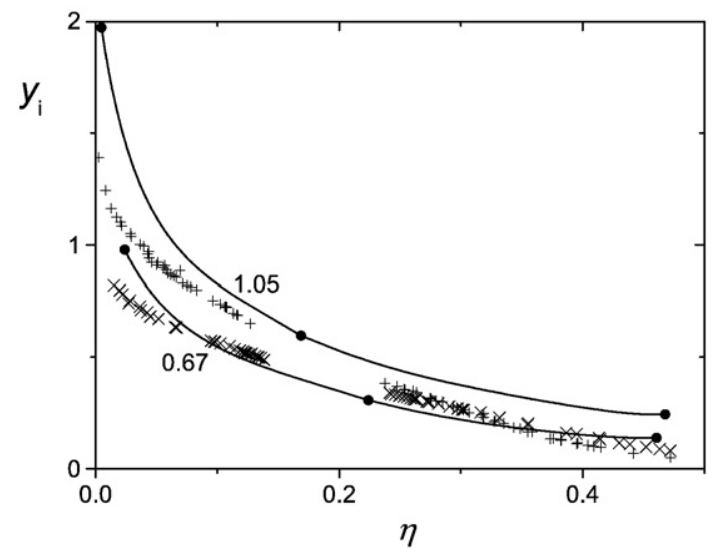

Fig. 8.3. Comparison of simulation binodals for $q_{R}=0.67$ (crosses, [87]) and 1.05 (plusses, [87]) for excluded-volume chains with theoretical binodals for the same $q_{R}$ values. The closed circles on the theoretical binodals correspond, from left to right, with tpG, cp, and tpL.

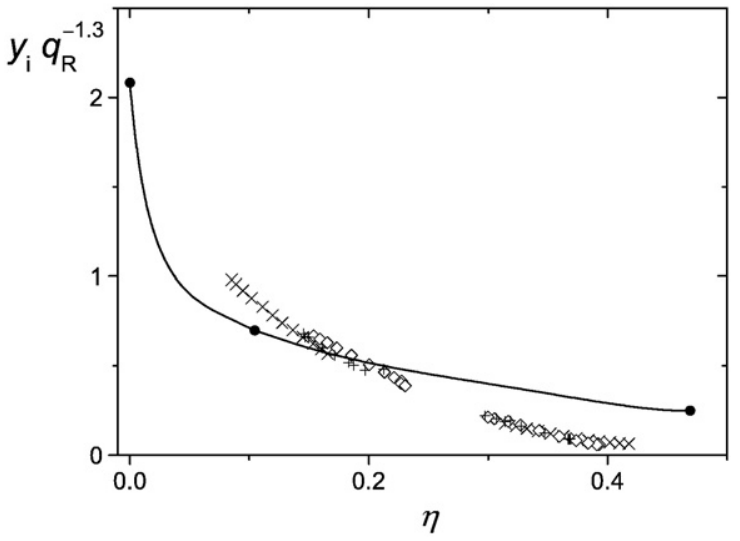

Fig. 8.4. Comparison of simulation binodals in the protein limit [40] for $q_{R}=3.86$ (crosses), 5.58 (plusses), and 7.78 (diamonds) for excluded-volume chains with theoretical binodals for the same $q_{R}$ values. Along the vertical axis we did not plot $y_{i}=\phi / \varphi_{\text {ov }}$ (as in Fig. 8.3) but $y_{i} q_{R}^{-1.3}$ so that all binodals for different $q_{R}$ collapse onto a single curve. The closed circles on the theoretical binodal correspond, from left to right, with tpG, cp, and tpL.

in the protein limit. Similarly, $\left(y_{i}\right)_{g}^{t} q_{R}^{-1.3}(\approx 2.07), y_{i}^{c} q_{R}^{-1.3}(\approx 0.70)$, and $\left(y_{i}\right)_{l}{ }_{l} q_{R}^{-1.3}(\approx 0.24)$ should be independent of $q_{R}$. So we have a universal binodal $y_{i} q_{R}^{-1.3}$ vs $\eta$, which is shown as the solid curve in Fig. 8.4. In the middle part this binodal coincides more or less with the simulation data but at the gas side it seems to be steeper than in the simulation, whereas at the liquid side it lies above the simulation points. The reason for these deviations are not clear at present; they could lie in some deficiency in our model, in the simulations, or in both. In the next section we shall see that there are indications that $\eta^{c}$ in the simulations for high $q_{R}$ is too high. Despite these uncertainties the overall agreement between theory and simulations is satisfactory.

\subsection{Critical points in simulations and other theories}

In Table 8.2 we collected as many theoretical and simulation data for the critical point as we could find in the literature; these are given in the third and fourth column of the table. All theories are for the excluded-volume limit; only in one case [36] the theory can also be applied to a theta solvent (bottom lines of the table). We first focus on the ev results.

In most cases $\eta^{c}$ is around 0.2 in the colloid limit, decreasing to around 0.1 in the protein limit. The density functional theory of Schmidt and Fuchs [36] give very small $\eta^{c}$ values which, moreover, do not become independent of $q_{R}$ in the protein limit; in that respect this theory is an exception as all other theories give (more or less) such a constant final level. The simulations of Bolhuis et al. [40,87] give the only example where $\eta^{c}$ for intermediate $q_{R}(0.67,1.05)$ is smaller $(\approx 0.18)$ than in the protein limit $(\approx 0.25)$; this suggests that the latter value is too high. A possible reason for the relatively large value for $\eta^{c}$ for large $q_{R}$ is the finite size of the polymer segments compared to the size of the HS. It has been shown by Tuinier [88] using extended FVT and by Paricaud et al. [89] using Wertheim perturbation theory that, for given $q_{R}$, increasing the relative size of segments leads to larger $\eta^{c}$ values. The latter theory [89] predicts that GL phase separation does not take place when the segments become very large.

As to $y_{\mathrm{i}}{ }^{c}$, the overall trend is that $y_{\mathrm{i}}{ }^{c}$ is around 0.2 in the colloid limit and increases with $q_{R}$. However, the predicted values in the theory of Pelisetto and Hansen [12] are consistently very low. Also the simulations by Chou et al. [41] give rather low $y_{i}^{c}$ values. Despite the more or less systematic variations between the various models, they all follow the $q_{R}^{1.3}$ scaling in the protein limit, although for the simulations of Chou et al. [41] this applies only for $q_{R}$ above 4 . The last four columns in Table 8.2 give the value of $y_{i}{ }^{C} q_{R}^{-1.3}$ in the protein limit: 0.11 [41], 0.14 [12], 0.20 [36], 0.34 [40], and 0.70 in our theory. So far, 
Table 8.2

Comparison of critical points in or model with simulations and other theories

\begin{tabular}{|c|c|c|c|c|c|c|c|c|c|}
\hline \multirow{2}{*}{$\frac{\mathrm{ev}}{\mathrm{Ref}}$} & \multirow[t]{2}{*}{$q_{R}$} & \multicolumn{2}{|c|}{$\operatorname{sim} /$ th } & \multicolumn{2}{|c|}{ present model } & \multicolumn{4}{|c|}{$y_{i}^{c} q_{R}^{-1.3}$} \\
\hline & & $\eta^{c}$ & $y_{i}^{c}$ & $\eta^{c}$ & $y_{i}^{c}$ & & & & \\
\hline [12] & 0.5 & 0.15 & 0.04 & 0.27 & 0.21 & & & & \\
\hline [36] & 0.5 & 0.22 & 0.18 & 0.27 & 0.21 & & & & \\
\hline [37] & 0.57 & 0.27 & 0.17 & 0.25 & 0.25 & & & & \\
\hline [87] & 0.67 & 0.19 & 0.40 & 0.22 & 0.30 & & & & \\
\hline [12] & 0.67 & 0.12 & 0.08 & 0.22 & 0.30 & & & & \\
\hline [56] & 0.8 & 0.15 & 0.35 & 0.20 & 0.37 & & & & \\
\hline [12] & 1 & 0.10 & 0.14 & 0.17 & 0.51 & & & & \\
\hline [33] & 1 & 0.20 & 0.58 & 0.17 & 0.51 & & & & \\
\hline [37] & 1 & $0.14-0.19$ & $0.15-0.22$ & 0.17 & 0.51 & & & & \\
\hline [36] & 1 & 0.11 & 0.23 & 0.17 & 0.51 & & & & \\
\hline [87] & 1.05 & 0.18 & 0.52 & 0.17 & 0.55 & & & & \\
\hline [12] & 1.67 & 0.08 & 0.4 & 0.13 & 1.15 & & & & \\
\hline [41] & 2.38 & 0.135 & 0.61 & 0.11 & 2.0 & 0.20 & & & \\
\hline [12] & 2.5 & 0.08 & 0.45 & 0.11 & 2.2 & & & & 0.14 \\
\hline [36] & 3 & 0.02 & 0.8 & 0.11 & 2.7 & & & 0.19 & \\
\hline [41] & 3.10 & 0.13 & 0.74 & 0.11 & 2.9 & 0.17 & & & \\
\hline [40] & 3.86 & 0.24 & 2.0 & 0.11 & 3.8 & & 0.35 & & \\
\hline [41] & 3.92 & 0.128 & 0.82 & 0.11 & 3.9 & 0.14 & & & \\
\hline [12] & 5 & 0.085 & 1.2 & 0.11 & 5.3 & & & & 0.15 \\
\hline [33] & 5 & 0.14 & 1.60 & 0.11 & 5.3 & & & & \\
\hline [41] & 5.08 & 0.122 & 0.89 & 0.11 & 5.4 & 0.11 & & & \\
\hline [40] & 5.58 & 0.25 & 3.2 & 0.11 & 6.1 & & 0.34 & & \\
\hline [41] & 6.06 & 0.122 & 1.14 & 0.11 & 6.8 & 0.11 & & & \\
\hline [40] & 7.78 & 0.25 & 4.9 & 0.11 & 9.4 & & 0.34 & & \\
\hline [12] & 10 & 0.095 & 2.8 & 0.11 & 13.0 & & & & 0.14 \\
\hline [36] & 10 & 0.004 & 4 & 0.11 & 13.0 & & & 0.20 & \\
\hline $\mathrm{mf}$ & & & & & & & & $y_{i}^{c} q_{R}^{-1}$ & \\
\hline [36] & 3 & 0.02 & 0.8 & 0.06 & 1.07 & & & 0.27 & \\
\hline [36] & 10 & 0.003 & 3 & 0.06 & 3.6 & & & 0.30 & \\
\hline
\end{tabular}

there are hardly any data to check which numerical prefactor would follow from experiment; the only good-solvent protein-limit result in Table 8.1 is that for (aggregated) whey protein/polysaccharide [83], which gives a value close to that in our theory. The other protein-limit experimental data in Table $8.1[84,85]$ are for near-theta conditions, where there is approximate agreement with our mf-version.

The bottom lines of Table 8.2 compare the theta-predictions of Schmidt and Fuchs [36] for $q_{R}=3$ and 10 with our model. The $\eta^{c}$ values seem to be irrealistically low (although the trend that mf gives a lower $\eta^{c}$ than ev is there). On the other hand, the $y_{\mathrm{i}}^{c}$ values are rather close to those in our model, and the value of $y_{i}{ }^{c} q_{R}{ }^{-1}$ is around 0.3 , where we predict 0.35 .

Sear [90] proposed a Flory-Huggins-type mean-field theory of a mixture of hard spheres plus chains described as connected blobs and gave predictions for the phase behavior of (small) hard spheres and (long) polymers. The author made a comparison with the simulation data [40] in Table 8.2 for the critical point. His conclusion is that both the polymer concentration $y_{i}^{c}$ and the colloid concentration $\eta^{c}$ in his theory are too low by roughly an order of magnitude. Our 'var' or (GFVT) model is much closer to the simulation data in this respect.

\subsection{Free volume fraction}

A remaining question is whether the expression for the free volume fraction $\alpha$ from scaled-particle theory (Eqs. (2.5)-(2.7), also used by and Aarts et al. [30]) is accurate for our 'var' version of the osmotic equilibrium theory, where $q$ decreases with increasing polymer concentration (for 'fix', where $q$ is constant, it has been verified by simulations [29]). To our knowledge this has never been tested. From recent MC simulations by Fortini et al. [91], which authors use the same method as Bolhuis et al. [87], we can deduce the free volume fraction as the ratio $\phi / \varphi$ or $y_{i} / y$ (see Fig. 7a,b in [91]) for a size ratio $q_{R}=1.05$. These simulation results are plotted in Fig. 8.5 as the open circles. Our theoretical 'var' prediction is given as the solid curve. The agreement is excellent for volume fractions below $\eta=0.25$, while it starts to deviate somewhat for larger colloid volume fractions. This implies that we might expect some deviations for binodal points at large volume fractions. As we have seen in Figs. 8.3 and 8.4 these actually do appear. We note that these simulations are not exact as they are based on the Gaussian Core model [38,39]. It would be interesting to obtain $\alpha=\phi / \varphi$ from MC simulations of HS plus selfavoiding walks [40] in the future to test the scaled-particle expression, also for other $q_{R}$ values.

\subsection{Tie lines}

We give one example of experimental tie lines, for the eye-lens protein $\gamma S$-crystalline in a PEO1450-water system [81]. The bottom

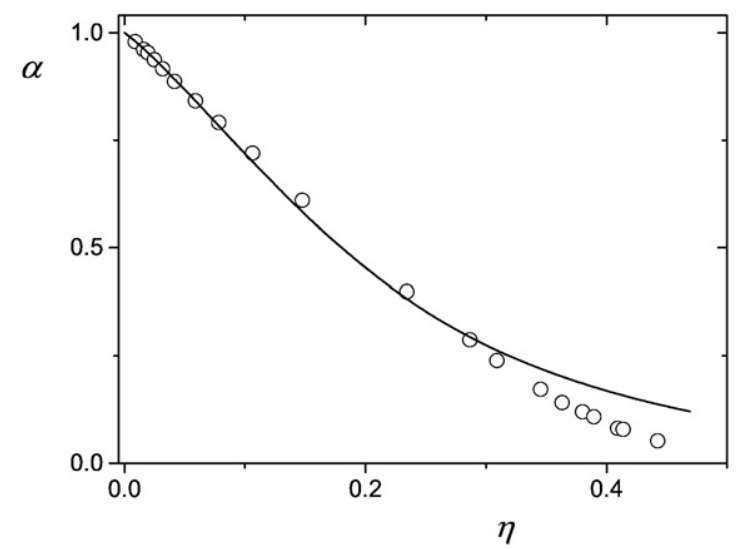

Fig. 8.5. Comparison of the free volume fraction $\alpha$ for $q_{R}=1.05$ from MC simulations (circles, [91]) with 'variable $q$ ' predictions (solid curve). 


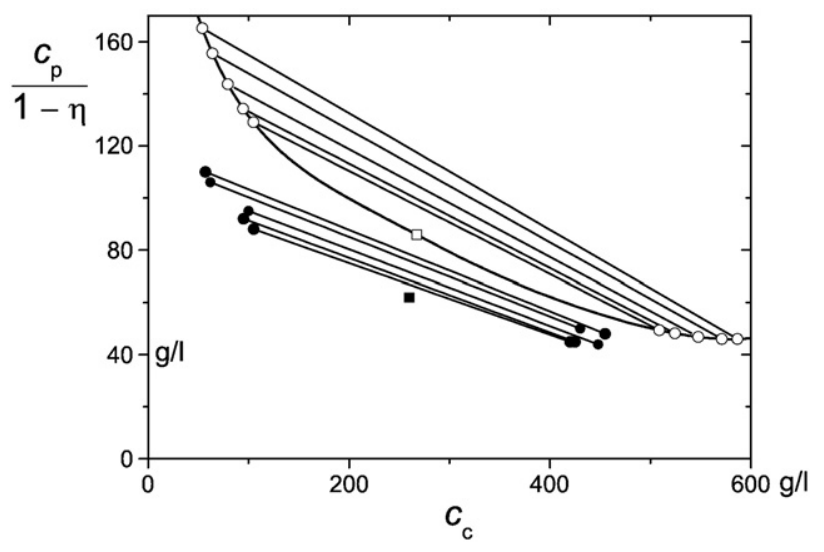

Fig. 8.6. Experimental tie-lines for $q_{R}=0.87$ ([81], bottom part) with the theoretical binodal plus tie-lines (top part) for the same $q_{R}$. The filled square is the estimated experimental $\mathrm{cp}$, the open square is the theoretical $\mathrm{cp}$.

part of Fig. 8.6 (the straight lines connecting the solid circles) gives the experimental tie lines in the coordinates $c_{p} /(1-\eta)$ versus $c_{c}$, where $c_{p}$ and $c_{c}$ are the polymer and colloid concentrations in $\mathrm{g} / \mathrm{l}$. The solid square in the figure is the estimated critical point in these coordinates.

The top part of Fig. 8.6 shows our theoretical prediction for the binodal and five tie lines; the binodal points at the gas side were chosen at (more or less) the same colloid concentrations as in the experiment. In order to convert the theoretical $\eta$ towards $c_{c}$ we need the specific volume $v_{\mathrm{c}}$ of the protein: $\eta=c_{c} v_{c}$, with $v_{c}=0.71 \mathrm{ml} / \mathrm{g}$. For obtaining the polymer concentration $c_{p}$ from $\phi=c_{p} / c_{\mathrm{ov}}$ we used $c_{\mathrm{ov}}=152 \mathrm{~g} / \mathrm{l}$ [81] for the overlap concentration.

Comparing the experimental and theoretical data in Fig. 8.6, we see that our theory overestimates the binodal polymer concentrations in the gas branch by some $25 \%$ (see also Table 8.1 , [81]), and the liquid end of the tie lines is situated at somewhat higher colloid concentrations. Apart from that the overall picture in theory and experiment is nearly the same. The slope of the tie lines is slightly higher in the theoretical model but this is only a minor effect. It seems that our model captures the main trends reasonably well, considering also the uncertainty in the conversion factors.

\subsection{Triple points}

Information about triple points is extremely scarce in the literature. We could find only one published example where the liquid composition of the triple point is reported [92]. These results, for three $q_{R}$ values in the system PMMA/PS/decalin are given in the first three columns in Table 8.3.

In order to interpret these data, we realize that in Table 8.1 this system was found to be a borderline case between ev (good solvent) and $\mathrm{mf}$ (theta solvent); this is why we give both the ev predictions (columns 4-5) and those for mf (columns 6-7).
When we take the experimental $q_{R}$ values (column 1) for granted, our theory predicts a stable liquid state only for $q_{R}>q_{R}^{*}$, where $q_{R}^{*}=$ $0.388(\mathrm{ev})$ or $q_{R}^{*}=0.337(\mathrm{mf})$. This implies that for ev and $\mathrm{mf}$ there is no stable liquid for $q_{R}=0.24$, and for ev it exists only for $q_{R}=0.57$. The predictions in Table 8.3 (top part) give a value of $\eta_{l}{ }^{t}$ quite close to the experimental value, whereas $\left(y_{i}\right)_{l}^{t}$ is about the same as (ev) or somewhat lower than ( $\mathrm{mf}$ ) the experimental values. In both cases it is unsatisfactory that for the lowest (experimental) $q_{R}$ no direct comparison with theory is possible.

In Section 8.1 we concluded that $q_{R}^{*}$ for a polydisperse system is probably smaller than for a monodisperse system. This implies that the 'polydisperse' data for the experimental $q_{R}$ in Table 8.3 might be interpreted by 'monodisperse' theory with a higher $q_{R}$ (th) than $q_{R}$ (exp). In the bottom part of Table 8.3 we did this by assuming that the experimental $\eta_{l}^{t}$ (which seems to be rather accurate) can be used as an input into the theoretical model. We then calculate which $q_{R}($ th) we need to give this $\eta_{l}{ }_{l}^{t}$ and compute also the corresponding $\left(y_{i}\right)_{l}{ }_{l}$. The result is shown in the last three lines of the table: columns $4-5$ for ev and columns 6-7 for mf. For $q_{R}(\exp )=0.24$ we then find a $q_{R}($ th $)$ which is just above $q_{R}^{*}$, and for $q_{R}(\exp )=0.37$ we need for ev a slightly higher theoretical value $(0.43)$, whereas for $\mathrm{mf}$ this value is 0.366 , basically the same as in experiment. The values for $\left(y_{i}\right)_{l}^{t}($ th $)$ are very close to the experimental values for ev, and slightly lower in mf. All in all, the theoretical description is rather close to these experimental tp data.

\subsection{A complete phase diagram for $q_{R}=1$}

We conclude this chapter with an example where the filled square was explored experimentally [43]. In this study the colloid concentration $\eta$ was varied from very low values to nearly close-packing, and the (internal) polymer concentration $y_{i}$ from very dilute to values well above overlap. For each $\eta, y_{i}$ combination the state of the system was monitored. For certain $\eta, y_{i}$ values two-phase coexistence (GL, GS, FS) was found, and for very specific $\eta, y_{i}$ combinations three-phase coexistence was encountered. Fig. 8.7 gives these experimental results, for nearly monodisperse PMMA colloids $(a \approx 130 \mathrm{~nm})$ and PS $(R \approx 125 \mathrm{~nm})$ in a mixed solvent of cis-decalin and tetralin (to match the refractive index of the particles). The solvency of this mixture for PS is believed to be moderately good.

The open circles in Fig. 8.7 indicate the compositions where one (fluid) phase occurred. Crosses correspond to two-phase GL coexistence and plusses to two-phase GS coexistence. The most interesting region in the phase diagram is given by the (open) triangles. Here three phases (G,L,S) occurred simultaneously. We confront these experimental results with the theoretical predictions of the 'fix' and 'var' models. In both cases we calculate the GL, GS and LS binodals $y_{i}(\eta)$, and the triple region where three-phase coexistence is expected.

The dashed curve in Fig. 8.7 gives the 'fix' GL binodal; the full square on this curve is the 'fix' critical point. This binodal ends to the left at the $\mathrm{G}$ side of the triple point (not visible in Fig. 8.7, it is situated at $y_{i} \approx 6$ at very low $\left.\eta\right)$ and to the right at the L-side $\left(y_{i} \approx 0\right.$ at $\left.\eta=0.49\right)$. It

Table 8.3

Liquid composition of the triple point

\begin{tabular}{|c|c|c|c|c|c|c|}
\hline \multirow[b]{2}{*}{$q_{R}$} & \multicolumn{2}{|c|}{ exp } & \multicolumn{2}{|c|}{ ev } & \multicolumn{2}{|c|}{$\mathrm{mf}$} \\
\hline & $\overline{\eta_{l}^{t}}$ & $\left(y_{i}\right)_{l}^{t}$ & $\eta_{l}^{t}$ & $\left(y_{i}\right)_{l}^{t}$ & $\eta_{l}^{t}$ & $\left(\mathrm{y}_{\mathrm{i}}\right)_{l}^{t}$ \\
\hline 0.24 & 0.333 & 0.13 & - & - & - & - \\
\hline 0.37 & 0.404 & 0.13 & - & - & 0.409 & 0.067 \\
\hline \multirow[t]{5}{*}{0.57} & 0.444 & 0.1 & 0.452 & 0.108 & 0.470 & 0.048 \\
\hline & & & $q_{R}$ & & $q_{R}$ & \\
\hline & 0.333 & 0.13 & 0.389 & 0.149 & 0.338 & 0.096 \\
\hline & 0.404 & 0.13 & 0.426 & 0.112 & 0.366 & 0.063 \\
\hline & 0.444 & 0.1 & 0.525 & 0.104 & 0.425 & 0.050 \\
\hline
\end{tabular}

The experimental data are from Moussaïd et al. [92]. 


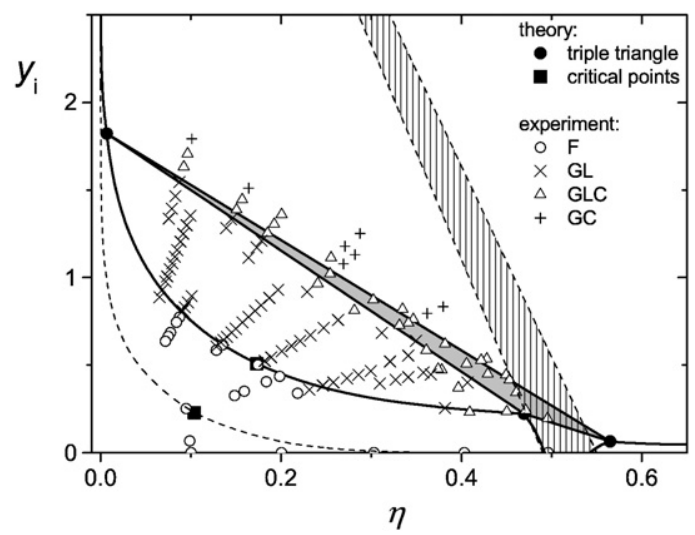

Fig. 8.7. Phase diagram of a colloid-polymer mixture with $q_{R} \approx 1$. Experimental observations are indicated by open symbols and crosses as listed within the figure. The theoretical triple triangle according to the 'fixed $q$ ' model (dashed lines) and our new 'variable $q$ ' model (solid lines) are also shown; filled circles correspond to the composition of coexisting GLC phases at the 'var' triple point. The theoretical FC, GL and GC binodals are indicated as the dashed ('fix') and solid ('var') curves, with the theoretical predictions for the critical point represented by filled squares.

is clear that this 'fix' binodal underestimates the experimental boundary between circles (F) and crosses (GL) severely; it lies entirely in the experimental one-phase region. The 'var' binodal (solid curve) works much better: nearly all the GL crosses are indeed found in the region above the binodal and below the lower edge of the triple triangle (the straight line connecting the $G$ and $L$ parts of the triple point). All the plusses for GS coexistence are above the upper edge of the triple triangle (connecting the $\mathrm{G}$ and $\mathrm{S}$ parts of the triple point). Ideally, all GLS triangles should be situated inside the triple triangle. Here we see a slight discrepancy between experiment and theory, as some GLS triangle are outside this region: the theoretical triple region seems to be narrower than the experimental one. However, the triple triangle according to the 'fix' model (dashed in Fig. 8.7) overestimates the three-phase coexistence region dramatically.

The remaining minor discrepancies between 'var' theory and experiment may be caused by a variety of effects. One of them is the small but finite polydispersity of the experimental system, which affects the phase behavior. A second is that the numerical constants used in $\Pi v(y)$ (Eq. (6.12)) and $q(y)$ (Eq. (6.17)), which were obtained from RG theory or simulations, might be slightly different for real systems; in principle these constants could be measured experimentally. A last issue is the solvency. The theoretical description in Fig. 8.7 is for a good solvent, whereas the experimental solvency is probably only 'moderately good' (i.e. a borderline case). When the theoretical theta ( $\mathrm{mf}$ ) version is used, the triple triangle widens somewhat at the liquid side (which improves the agreement with experiment), but the gas side of this triple triangle is now too low $\left(y_{i} \approx 1.2\right.$ instead of 1.9 in Fig. 8.7). We would need a theory for marginally good solvents to improve the situation. So far, such a theory is not available.

Nevertheless, it is clear that the 'var' theory is in nearly quantitative agreement with available experimental data and constitutes a major improvement on the classical 'fix' model.

\section{Concluding remarks}

In this paper we discussed the phase behavior of a mixture of colloids, polymer chains, and solvent. We focus on non-adsorbing polymer chains; for adsorbing polymer the picture is completely different [93]. From a fundamental point of view the statistical thermodynamics of such a three-component mixture constitutes a major challenge, especially when the polymer chains are self-avoiding. Even in relatively simple thermodynamic perturbation theory [35] the calculation of twophase equilibria (binodals) requires extensive numerical work, and three-phase equilibria (triple points) are even more difficult.
We show in this paper that free-volume theory (FVT), based upon very simple and transparent concepts, is a useful tool for describing the phase behavior. We show how to calculate binodal points, triple points, critical points, and the critical endpoint with minimal computational effort. Hence, the entire phase diagram for the full range of polymer and colloid concentrations is available. The original FVT [26], where the solvent component is ignored, can be simply extended to include this component. In doing so the parameter combination $\Pi v$, where $\Pi$ is the polymer osmotic pressure and $v$ the colloid volume, emerges as the natural thermodynamic parameter. Along the binodals $\Pi v$ is nearly the same for ideal chains and for excluded-volume chains for which the depletion thickness decreases with increasing polymer concentrations.

The phase behavior depends strongly on the size ratio $q_{R}=R / a$ between polymer coils (radius $R$ ) and colloid particles (radius $a$ ). In the colloid limit $\left(q_{R}<<1\right)$ the depletion thickness $\delta=\delta_{0}$ is constant $(\approx R)$ and $\Pi=\Pi_{0}=\varphi / N$ (ideal chains); this is the original version of FVT [26]. For higher $q_{R}$ the ratios $\delta / \delta_{0}$ and $\Pi / \Pi_{0}$ depend on the polymer concentration $\varphi$ (or, more precisely, on the ratio $y=\varphi / \varphi_{\text {ov }}$ ). The extension of FVT in this direction for good and theta solvent conditions is straightforward; we can now also describe the protein limit $\left(q_{R}>1\right)$. We used numerical prefactors for the $y$-dependence in the semidilute limit derived from renormalization-group theory (for $\Pi$ ) or from computer simulations (for $\delta$ ). However, these numerical constants are, in principle, also accessible from well-designed experiments for the concentration dependence $\Pi(\varphi)$ and $\delta(\varphi)$.

It turns out that for this generalized FVT (GFVT) the most convenient parameter for the polymer concentration is $Y=y q_{R}^{-1 / \gamma}$, with $\gamma=1$ in mean field ( $\mathrm{mf}$ ) and $\gamma=0.77$ for excluded-volume chains (ev). For critical points (cp) and triple points (tp) and along the binodals $Y$ is always of order unity and it varies only weakly with $q_{R}$. In the protein limit, $Y^{\mathrm{cp}}$ and $Y^{\mathrm{tp}}$ (and $Y$ along the binodals) become independent of the size ratio $q_{R}$.

We summarize the main theoretical predictions as follows. The critical endpoint (cep), where tp and cp coincide, is situated at $q_{R}=$ $q_{R}^{*}=0.39$ and $Y=Y^{*}=1.46$ (or $y=y^{*}=0.43$ ) for ev (good solvent). For high $q_{R}$ we find a constant level $Y_{\infty}^{c}=0.97$ (cp) and $Y_{\infty}^{t}=2.08$ (tp); this implies that $y^{c}$ and $y^{t}$ diverge as $y_{\infty}^{c}=0.97 q_{R}^{1.3}$ and $y_{\infty}^{t}=2.08 q_{R}^{1.3}$. (For mf these numbers are slightly different.) Liquid is only stable for $q_{R}>q_{R}^{*}$ and in the (polymer) concentration range $Y^{c}<Y<Y^{t}$ or $y^{c}<y<y^{t}$. The liquid window is thus narrow: it covers (at most) a factor 2 in (external) polymer concentration. For high $q_{R}$ the polymer concentrations needed for GL demixing are far above the overlap concentration, by a factor $q_{R}^{1.3}$ (which is above 8 for $q_{R}$ above 5 ). At such high polymer concentrations the solutions are very viscous, and demixing is extremely slow. This might explain the experimental finding [83] that dispersions of very tiny particles are nearly always co-soluble with long polymer chains.

The value of $Y^{*}$ or $y^{*}$ at the cep may be converted to the strength $\varepsilon^{*}$ of the pair interaction; the result is $\varepsilon^{*} \approx 2 k T$. The relative range $q_{R}^{*}$ is around 1/3: in the cep the range is one third of the particle radius. These numbers are nearly the same for different models. Original FVT with a 'fixed' depletion thickness $\delta$, generalized FVT (i.e., GFVT) with 'variable' $\delta$ (for both ev and $\mathrm{mf}$ ), but also quite different systems like hard spheres with a Yukawa attraction [64] or particles with a Lennard-Jones-type pair potential [72] all give this critical strength $2 k T$ and relative range $1 / 3$. This suggests a universal principle for the existence condition of a stable liquid. For a higher strength and/or shorter range the crystalline solid is the preferred situation for the condensed phase.

Also the width of the liquid window may be expressed in terms of the interaction strength ( $\varepsilon^{c}$ for $\mathrm{cp}$ and $\varepsilon^{t}$ for tp). At high $q_{R}, \varepsilon^{t}-$ $\varepsilon^{t} \approx 1.4 k T$ for a colloid/polymer mixture, which again points to a narrow window. For a Yukawa system the liquid window is even narrower: $\varepsilon^{t}-\varepsilon^{t} \approx 0.6 k T$ at most. This illustrates the subtle balance for the competition between fluid-solid and fluid-fluid demixing. 
Accordingly the liquid state is delicate, at least for strictly monodisperse particles. For experimental (polydisperse) particles the liquid window might be wider because now the alternative condensed phase (i.e., a crystal) is less favorable.

We compared our generalized FVT extensively with experiments, with computer simulations, and with other theoretical approaches. In most cases we find (semi)quantitative agreement, which is gratifying. Remaining discrepancies are most probably related to some polydispersity of the experimental dispersions or to (weak) adsorption [86]. Close to the critical point slight deviations might be due to the fact that scaled-particle theory, which lies at the basis of (G)FVT, is mean-field like; this leads to deviations near the critical point [56]. These deviations are not expected to be dramatic for long-ranged attractions [94].

A particularly interesting aspect is our scaling result $y \sim q_{R}^{1 / \gamma}$ along the protein-limit binodals. This scaling is corroborated by simulations and other theoretical models (although this scaling was not recognized in the literature published so far). Experimental data for the protein limit are too scarce to check this scaling experimentally. We hope that our results will lead to more systematic studies in this direction.

We have presented in this paper not only numerical phase diagrams, but also simple explicit analytical expressions for critical points, triple points, and binodals. These expressions should enable experimentalists to plan new experiments, as the parameter range for phase separation follows directly from these simple equations. In this way it becomes possible to systematically vary the polymer chain length, the particle radius, and the solvency. This latter aspect could be introduced by using polymers (e.g., PNIPAM) for which the solvency depends strongly on the temperature.

We formulated theory for either a good solvent or theta conditions. This covers the relevant range of solvencies almost entirely. Only for solvents on the edge between theta and good solvents (Flory-Huggins parameter $\chi$ about $0.48-0.49)$ and for bad solvents $(\chi>0.5)$ do we not have a proper description.

Our generalization of FVT, possible by including the correct dependencies for the depletion thickness and the osmotic pressure on polymer concentration for interacting chains, shows that freevolume theory can be modified to describe non-ideality. Further extensions to more complex systems seem possible, as demonstrated recently for charged colloids [95]. It would be challenging to also include sticky spheres, polymers in a (slightly) bad solvent, and polydispersity effects.

\section{Acknowledgements}

First of all we are greatly indebted to Sasha Skvortsov for collaboration on analytical theory that yielded ingredients such as polymer depletion thickness and osmotic pressure as a function of polymer concentration. Part of this work is published in other papers but constitutes an important basis of our 'var' theory. We thank Henk Lekkerkerker, Dirk Aarts, Frans Leermakers, Christoph Gögelein, Peter R. Lang, Wilson Poon, Klaas Besseling and Erich Eisenriegler for fruitful discussions and comments on various aspects of this work. Andrea Fortini and Peter Bolhuis are thanked for providing computer simulation data. Finally, Martien Cohen Stuart encouraged us to join forces on depletion in 2002, for which we owe him a salute.

\section{References}

[1] Einstein A. Investigations on the Theory of the Brownian Motion. Dover Publications; 1956.

[2] von Smoluchowski M. Ann Phys 1906;21:756.

[3] Perrin J. Atoms. London: Constable \& Company; 1916.

[4] Yethiraj A, van Blaaderen A. Nature 2002;421:513.

[5] Levelt Sengers J. How fluids unmix, Edita KNAW. The Netherlands: Amsterdam; 2002.

[6] Poon WCK, Pusey PN, Lekkerkerker HNW. Colloids in suspense. Physics World; 1996. p. 27-32. April.

[7] Ilett SM, Orrock A, Poon WCK, Pusey PN. Phys Rev E 1995;51:1344.

[8] Leal-Calderon F, Bibette J, Biais J. Europhys Lett 1993;23:653.
[9] Faers MA, Luckham PF. Langmuir 1997;15:2922.

[10] Meijer EJ, Frenkel D. J Chem Phys 1994;100:6873.

[11] Dijkstra M, Brader JM, Evans R. J Phys: Condens Matter 1999;11:10079.

[12] Pelissetto A, Hansen JP. Macromolecules 2006;39:9571.

[13] Fuchs M, Schweizer KS. Europhys Lett 2000;51:621.

[14] Louis AA, Bolhuis PG, Hansen JP, Meijer EJ. Phys Rev Lett 2000;85:2522.

[15] Sear RP. Phys Rev Lett 2001;86:4696.

[16] Odijk T. J Chem Phys 1997;106:3402.

[17] Asakura S, Oosawa F. J Chem Phys 1954;22:1255.

[18] Asakura S, Oosawa F. J Pol Sci 1958;33:183.

[19] Sieglaff CL. J Pol Sci 1959;41:319.

[20] Vrij A. Pure Appl Chem 1976;48:471.

[21] de Hek H, Vrij A. J Colloid Interface Sci 1981;84:409.

[22] Long JA, Osmond DWJ, Vincent B. J Colloid Interface Sci 1973;42:545.

[23] Vincent B, Edwards J, Emmett S, Croot R. Colloids Surf 1988;31:267.

[24] Gast AP, Hall CK, Russel WB. J Colloid Interface Sci 1983;96:251.

[25] Lekkerkerker HNW. Colloids Surf 1990;51:419.

[26] Lekkerkerker HNW, Poon WCK, Pusey PN, Stroobants A, Warren PB. Europhys Lett 1992;20:559.

[27] Poon WCK. J Phys: Condens Matter 2002;14:R859.

[28] Moncho-Jorda A, Louis AA, Bolhuis PG, Roth R. J Phys: Condens Matter 2003;15: S3429.

[29] Dijkstra M, van Roij R, Roth R, Fortini A. Phys Rev E 2006;73:041404.

[30] Aarts DGAL, Tuinier R, Lekkerkerker HNW. J Phys: Condens Matter 2002;14:7551.

[31] Chatterjee AP, Schweizer KS. J Chem Phys 1998;109:10464 ibid 109:10477.

[32] Fuchs M, Schweizer KS. Phys Rev E 2001;64:021514.

[33] Fuchs M, Schweizer KS. J Phys: Condens Matter 2002;14:R239.

[34] Ramakrishnan S, Fuchs M, Schweizer KS, Zukoski CF. Langmuir 2002;18:1082.

[35] Rotenberg B, Dzubiella J, Hansen JP, Louis AA. Mol Phys 2004;102:1.

[36] Schmidt M, Fuchs M. J Chem Phys 2002;117:6308.

[37] Schmidt M, Denton AR, Brader JM. J Chem Phys 2003;118:1541.

[38] Louis AA, Bolhuis PG, Meijer EJ, Hansen JP. J Chem Phys 2002;116:10547.

[39] Louis AA, Bolhuis PG, Meijer EJ, Hansen JP. J Chem Phys 2002;117:1893.

[40] Bolhuis PG, Louis AA, Meijer EJ. Phys Rev Lett 2003;90:068304.

[41] Chou CY, Vo TTM, Panagiotopoulos AZ, Robert M. Physica A 2006;369:275.

[42] Fleer GJ, Tuinier R. Phys Rev E 2007;76:041802.

[43] Tuinier R, Smith PA, Poon WCK, Egelhaaf SU, Aarts DGAL, Lekkerkerker HNW, Fleer GJ. Europhys Lett 2008;82:68002.

[44] Schäfer L. Excluded-volume effects in Polymer Solutions. Berlin: Springer Verlag; 1999.

[45] Fleer GJ, Skvortsov AM, Tuinier R. Macromolecules 2003;36:7857.

[46] Fleer GJ, Skvortsov AM, Tuinier R. Macromol Theory \& Simulations 2007;16:531.

[47] Reiss H, Frisch HL, Lebowitz JL. J Chem Phys 1959;31:369. Reiss H. J Phys Chem 1959;96:4736.

[48] Widom B. Science 1967;157:375.

[49] Carnahan NF, Starling KE. J Chem Phys 1969;51:635.

[50] Hall CK. J Chem Phys 1972;52:2252.

[51] Frenkel D, Ladd AJC. J Chem Phys 1984;81:3188.

[52] Wood WW, Jacobson JD. J Chem Phys 1957;27:1207.

[53] Alder BJ, Wainwright TE. J Chem Phys 1957;27:1208.

[54] Hoover WG, Ree FM. J Chem Phys 1968;49:3609.

[55] Rowlinson JS, Widom B. Molecular Theory of Capillarity. Clarendon Press; 1989. Stanley HE. Introduction to Phase Transitions and Critical Phenomena. Oxford: Oxford University Press; 1988.

[56] Vink RLC, Horbach J. J Chem Phys 2004;121:3253.

[57] Flory PJ. Principles of polymer chemistry. Ithaca, New York: Cornell University Press; 1953.

[58] Eisenriegler E. J Chem Phys 1983;42:1052.

[59] Hanke A, Eisenriegler E, Dietrich S. Phys Rev E 1999;59:6853.

[60] de Gennes PG. Scaling Concepts in Polymer Physics. Ithaca: Cornell University Press; 1979.

[61] Le Guillou JC, Zinn-Justin J. Phys Rev Lett 1977;39:95.

[62] Fleer GJ, Tuinier R. Physica A 2007;379:52.

[63] Hagen MHJ, Frenkel D. J Chem Phys 1994;101:4093.

[64] Tuinier R, Fleer GJ. J Phys Chem B 2006;110:20540.

[65] Lekkerkerker HNW. Physica A 1997;244:227.

[66] Tejero CF, Daanoun A, Lekkerkerker HNW, Baus M. Phys Rev Lett 1994;73:752.

[67] Tang Y, Lu BCY. J Chem Phys 1993;99:9828.

[68] Tang Y, Lin ZY, Li YG. J Chem Phys 2005;122:184505.

[69] Dijkstra M. Phys Rev E 2002;66:0214402.

[70] Tuinier R, Fleer GJ. Macromolecules 2004;37:8764.

[71] Tuinier R, Aarts DGAL, Wensink HH, Lekkerkerker HNW. Phys Chem Chem Phys 2003;5:3707.

[72] Vliegenhart GJ, Lodge JF, Lekkerkerker HNW. Physica A 1999;263:378.

[73] Ramakrishnan S, Fuchs M, Schweizer KS, Zukoski CF. J Chem Phys 2002;116:2201.

[74] Tuinier R, de Kruif CG. J Chem Phys 1999;110:9296.

[75] Bolhuis PG, Kofke D. Phys Rev E 1996;54:634.

[76] Fasolo M, Sollich P. J Chem Phys 2005;122:074904.

[77] Fasolo M, Sollich P. J Phys: Condens Matt 2005;17:797.

[78] Lynch I, Cornen S, Piculell L. J Phys Chem 2004;108:5443.

[79] Bodnár I, Oosterbaan WD. J Chem Phys 1997;106:7777.

[80] Aarts DGAL. J Phys Chem B 2005;109:7407.

[81] Annunziata O, Ogun O, Benedek GB. Proc Natl Ac Sci, USA 2003;100:970.

[82] de Hoog EHA, Lekkerkerker HNW. J Phys Chem B 1999;103:5274.

[83] Tuinier R, Dhont JKG, de Kruif CG. Langmuir 2000;16:1497.

[84] Zhang ZX, van Duijneveldt JS. Langmuir 2006;22:63. 
[85] van Duijneveldt JS, Mutch K, Eastoe J. Soft Matter 2007;3:155.

[86] Wijting WK, Knoben W, Besseling NAM, Leermakers FAM, Cohen Stuart MA. Phys Chem Chem Phys 2004;6:4432.

87] Bolhuis PG, Louis AA, Hansen JP. Phys Rev Lett 2002;89:128302.

[88] Tuinier R. Eur Phys J E 2001;10:123.

[89] Paricaud P, Varga S, Jackson G. J Chem Phys 2003;118:8525.

[90] Sear RP. Phys Rev E 2002;66:051401.
[91] Fortini A, Bolhuis PG, Dijkstra M. J Chem Phys 2008;128:024904.

[92] Moussaïd A, Poon WCK, Pusey PN, Soliva MF. Phys Rev Lett 1999;82:225.

[93] Fleer GJ, Cohen Stuart MA, Scheutjens JMHM, Cosgrove T, Vincent B. Polymers at Interfaces. London: Chapman \& Hall; 1993.

[94] Fu D, Li Y, Wu J. Phys Rev E 2003;68:011403.

[95] Fortini A, Dijkstra M, Tuinier R. J Phys: Condens Matter 2005;17:7783. 